

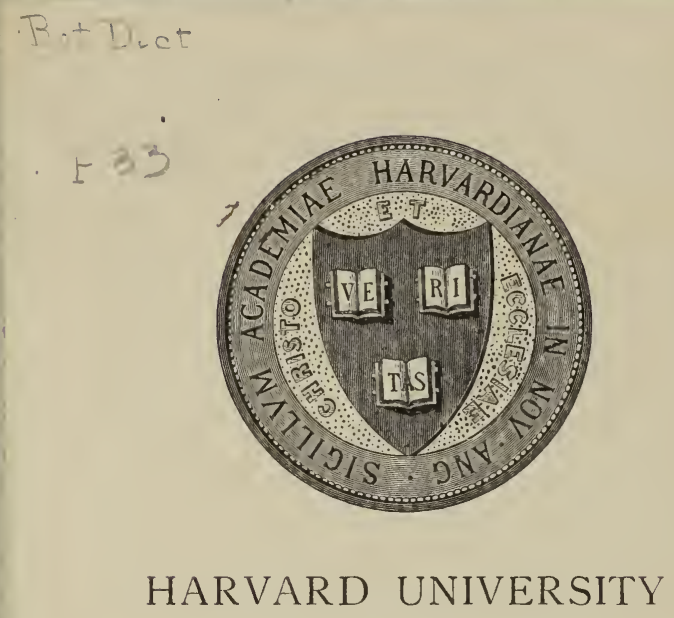

L I B R A R Y

OF TIIE

\section{GRAY HERBARIUM}

Received 


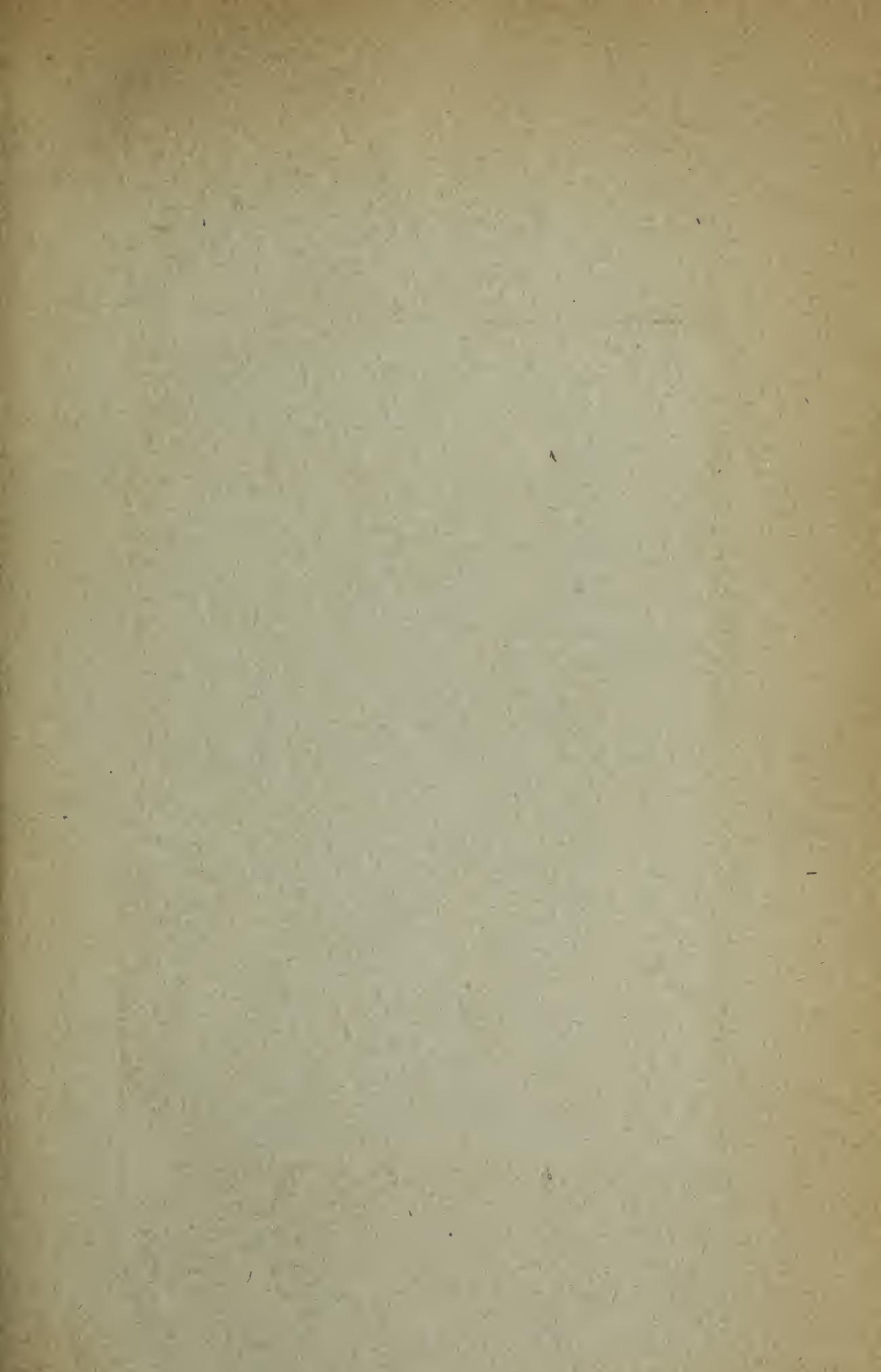





\section{Volksbenennungen}

der brasilianischen Pflanzen und Produkte derselben in brasilianischer (portugiesischer) und von der Tupisprache adoptirten Namen.
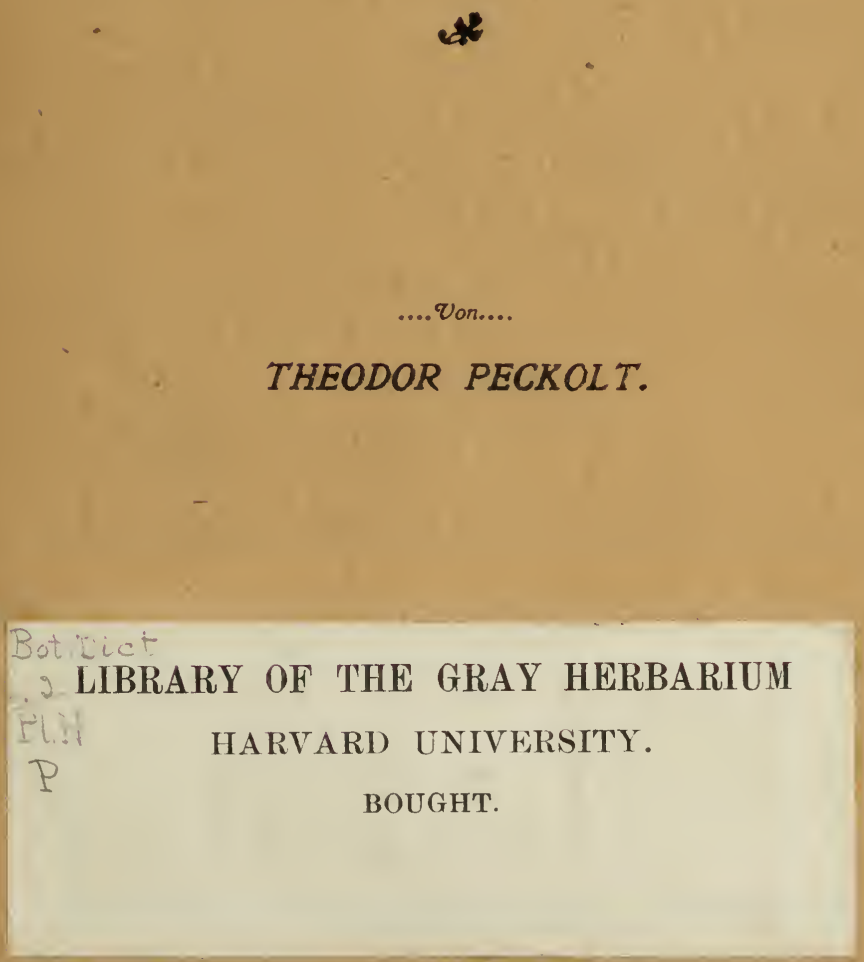

MILWAUKEE,

Pharmaceutical Review Publishing Co.

1907 . 


\section{PUBLICATIONS \\ ...by the.... \\ Pharmaceutical Review Publ. Co.}

Pharmaceutical Review. Formerly the Pharmaceutische Rundschau of of New York City, established in December 1882 and edited up to December 1895 by Dr. Fr. Hoffmanu in the German language. Edited since Jauuary 1896 in the English language by Edward Kremers with the cooperation of a number of the ablest representatives of pharmaceutical science in the United States. With the January number it has entered upon the twenty-fifth year of service to American pharmacy. Published monthly,

$\$ 2.00$

Pharmaceutical Arcbives. From 1898 to 1903 incl. the Archives were published as a supplementary journal to the Review and contain the orgiual matter for those years. With the exception of a few numbers, these volumes can still be supplied at a cost of a dollar per volume or of $\$ 5.00$ for the six years. For the missing numbers advertisements will be inserted in the Review, free of charge, so that the purchaser of the set may secure them directly without commission or fee.

The Volatile Oils. This standard work, written by Drs. Gildemeister and Hoffmann under the auspices of Schimmel and Co. of Leipzig, is universally acknowledged to be the most authoritative and elaborate work on the subject. The English translation by Edward Kremers is a volume of 730 pages. The work is enhanced by four maps and numerous illustrations and is exellently bound.

$\$ 5.00$

The Follies of Science. This is the last literary production of the late Dr. H. Carrington Bolton and should prove of interest to every lover of the history of chemistry, alchemy, pharmacy and medicine. It is highly illustrated.

$\$ 2.00$

"In reading this bouk, if we are not sure whether we are reading romance or history, we are none the less impressed with the authors' keen appreciation of literary values, and we have no hesitation in stating that it is one of the most fascinating volumes, either closely or remotely connected with medical history, that we have read in a long time."

Med. Libr. \& Hlst. Journ., vol. 3, p. 303.

The Badger Pharmacist. An account of pharmacy, in all its branches and aspects, in the state of Wisconsin, edited and published by the pliarmacy students of the University of Wisconsin. The work is highly illustrated and should appeal to every badger whether a resident of the state at present or not.

$\$ 1.00$

Bookplates. A collection of pharmacentical book-plates, printed on heavy glazed paper, suitable for framing.

Fach, $\$ 0.10$

Exlibris of Hermann Gelder designed by Lor. M. Rheude.

" "E. A. Merck designed by B. Wenig.

“ " "Louis Merck designed by B. Wenig.

“ " "Pharm. Institute University of Bern designed by F. Widmann.

“ " $\quad$ O. A. Oesterlo designed by Mario Flückiger.

" "O. A. Oesterle designed by M. P.

" "Pharm. Soc. of Switzerland designed by F. Widmann.

" " Otto Suldter designed by J. Kaufmann.

" " " . O. Suidter designed by J. Kauffmann.

" " Fredk. Stearns.

"A. Tschirch designed by F. Widmann.

Portraits. A collection of portraits of scientists: pharmacists, chemists and botanists, printed on heavy glazed paper, suitable for framing.

Each, $\$ 0.10$

Model Pharmacies. A collection of designs for drug store equipments in accordance with the principles of Gothic, renaissance etc. architecture.

Each, $\$ 0.10$ 
Digitized by the Internet Archive in 2017 with funding from BHL-SIL-FEDLINK 


\section{Pharmaceutical Science Series.}

EDITED BY

EDWARD KREMERS.

MONOGRAPHS.

No. 15.

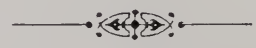

MILWAUKEE,

Pharmaceutical Review Publishing Co. 1907. 
0

\section{Volksbenennungen}

der brasilianischen Pflanzen und Produkte derselben in brasilianischer (portugiesischer) und von der Tupisprache adoptirten Namen.
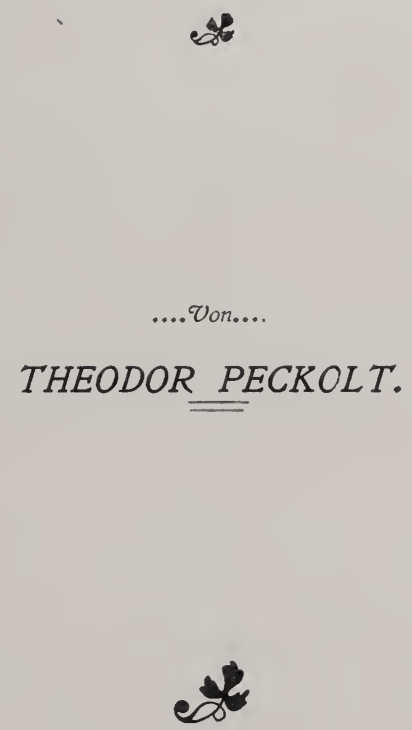

MILWAUKEE,

Pharmaceutical Review Publishing Co 1907. 
MAY 171909

verbarimen 


\section{Volksbenennungen der brasilianischen Pflanzen und Produkte derselben in brasilianischer (portu- giesischer) und der von der Tupisprache adoptirten Namen.}

By Dr. Theolor P'eckolt.

$\Lambda$ bacate. Frucht des Abacateiro.

A bacate do mato. Frucht von Salacia fluminensis Peyr. Hippocratacex. Grosse Frucht, $12 \mathrm{~cm}$. lang, 81/2 cm. Durchmesser. Pulpe essbar, die bitteren Samen als Tonicum, enthalten $22 \%$ fettes Oel und kryst. Bitterstoff (Salicidiu) $0.08 \%$.

A bacatiro. Abacatenbaum. Persea gratissima Gärtn. Lauracex. Frucht essbar, nahrhaft. Beere, Blüthen und Rinde arzneilich benutzt. Perseithaltig.

$\Lambda$ bacateiro ch iros o. Wohlriechender A. Persea meroneura Meissn.

Lauracex. Blätter und Rinde arzneilich benutzt.

A bacateiro cimarona. Persea laevigata H. B. Kth. Lauracex. A bacateiro do mato. Lauruscitriformis Vellos. Lauraceae. Frucht adstringens.

A acatú. Passavería obovata Mart. et Licht. Sapotaceae. Frucht schleimig, wird von den Indianern genossen.

Abacaxi. Varietät von Ananas sativus Schult. Bromeliaceae. Früchte sind grösser, aromatischer und bedeutend wohlschmeckender als die gewöhnliche Ananas; wird zum Unterschied von den Gärtnern Ananas sativus Schult. var. pyramidalis Don benannt. Man hat folgende Varietäten:

A bacaxi amarello. Gelbe A. var. pyramidalis aurea Don. $\Lambda$ bacaxi branco. Weisse $A$. var. pyramidalis alba Don. $A$ bacaxi vermelho. Rothe $A$. var. pyramidalis rubra Don. A bacaxi roxo. Violette $A$. var. pyramidalis violacea Don. A bacaxi verde. Grüne A. var. pyramidalis viridis Don.

A bacaxi de tingir. Färber Abacati, heisst auch: Gravata de tingir. Aechmea tinctoria Metz. Bromeliaceae. Das Decoct zum Geibfärben der Zeuge. 
A bajerú. Moquilea canomensis Mart. Rosaceae. Heisst auch: Goajerú. Samen essbar, wohlschmeckend, Rinde und Wurzel arzneilich benutzt.

A ban od. Mangue de praia.

A bará. Im Staate Bahia eine Lieblingsspeise. Klösse aus schwarzen

Bohnen, Dendé-Oel und Capsicum frutescens.

A baremo-temo. Pithecolobium A varemotemo Mart. Mimosae.

Rinde energisches Adstringens.

A bati-timbahy. Hymenaea Courbaril L. Caesalpineae, vide Jetahy. A bati. Oryza sativa Linn. Gramineae. Tupyname.

A bati-y. Grosskörniger Reis, heisst auch: Abati-opé und Abaxi-y. A batú. Das aus Maiskuchen gegohrene Getränk der Indianer.

A berêm. Gegohrene, dann in Bananenblättern geschmorte Maiskuchen der Indianer.

A biegna. Der durch Verwundung aus dem Stamme von Cecropia concolor Willd. ausfliessende Saft als Wundheilmittel. (Urticaceae.)

A biú. Lucuma Caimito A. DC. Sapotaceae. Heisst auch: Abi und Abi-abi; aus diesen Tupiwörtern ist wahrscheinlich das Wort abiú entstanden. Essbare, wohlschmeckende Frucht und ölreiche Samen. $11 \%$. Enthalten Lucumin.

A biú-rana. Falsche Abiú. Lucuma lasiocarpa A. DC. Sapotaceae. Heisst auch: Abiú sylvestre - Wilde A. Früchte werden von den Indianern genossen. Die Milch des Stammes liefert Guttapercha. Abobora. Gemeiner Kürbiss. Cucurbita pepo L. Cucurbitaceae. Die verschiedenen Varietäten l:aben folgende Benennungen: Abobora menina, $\Lambda$ boborachila, Jerimú, M.oyango und Porqueira.

A bobora d'agua. Wasserkürbiss. Lagenaria vuigaris Serr. Cucurbitaceae. Wenn unreif wird als Gemüse genossen; reif wird die harte Schale als Gefäss, die Fruchtpulpe und Samen arzneilich benutzt.

A bobora cabelluda. Zottiger Kürbiss. Cucurbita villosa Bl. Zur Nahrung.

Abobora carneira. Schafkürbiss. Cucurbita succada H. et B. Cucurbitaceae. Zur Nahrung.

A bobora crúa. Wohlriechender Kürbiss. Cucurbita ceratocreos $\mathrm{H}$. Wird nicht genossen, zum Wohlgeruch ins Zimmer gelegt, die Pulpe arzneilich benutzt.

A bobora doce. Süsser Kürbiss. Cucurbita melopepo L. Wird fast nie genossen, mehr als Gartenzierde, ist aber wohlschmeckend. 
A bobora de empigem. Flechtenkürbiss. Cucurbitil verrucosia L.

Wird zufolge der harten Schale wie Lagenaria zu Gefässen benutzt. Abobora farinhosa. Mehlkürbiss. Cucurbita firlinosia Bl. Als Speise benutzt.

A bobora grande. Grosser Kürbiss. (ucurbita maximal I)uch. Heisst auclı: Yarumú. Dient zur Speise und Viehfütterung.

Abobora mamma. Zitzen Kürbiss. Cucurbita mammeata Mol. Sehr wohlschmeckend.

Abobora melao. Melonenkürbiss. Cucurbita moschata Inch. Blïthen riechen moschusartig, werden ins Zeug gelegt. Frucht zur Speise.

A bobora ovos. Eierkürbiss. Cncurbita oviferal L. Dient zu Speise und Confect.

Abobreira do mato. Waldkürbisspflanze. Mehrere brasilianische Cucurbitaceen haben diesen Volksnamen als: Drupilna racenosa Mamo, Willbrandia drastica Mart. und hilbiscoides Mams, Melothria fluminensis Gard. und Cayaponia villosa Cogn. Von einigen werden die Früchte, von anderen die Wurzeln als Purganz benutzt.

Abobrinho do mato. Kleiner wilder Kürbiss. Trianosperma diversifolia C., Trianosperma ficifolia Mart. und Trianosperma Martiana Cog. Die Wurzeln als drasticum, die Blätter bei Keuchhusten. Alle diese Cucurbitaceen haben den Namen Kü̈rbiss (Abobora) zufolge der Blattähnlichkeit. Die Früchte sind klein.

A bricó de Parà. Paràaprikose. Mammea americana L. Guttiferae. Die grosse Frucht als Delikatesse, das Holz des Baumes zu Bauten.

Abricó do mato. Wilde Aprikose. Mimusops coriacea Miq. Sapotaceae. Grosser Baum, Bauholz. Frïchte werden genossen. Die Rinde und bitteren Samen arzneilich benutzt.

Abutua. Chondendron tomentosum Ruiz et Pav. Menispermaceae. Heisst auch: Butua, Pareira brava und Mai bôa. Die Wurzel ist officinell als Radix pareiræ bravæ.

Abutua miuda. Kleine Butua. Cocculus filipendula Mart. Menispermaceae. Wurzel als Antidot bei Schlangenbiss.

Acaia. Spondias purpurea Linn. var. venulosa Mart. Anacardiaceae. Heisst auch: Caja, Acaja und Ibá-metarà. Frïchte essbar. Bauholz. Acaia-ca. Cedra Velloziana Roin. Meliaceae. Heisst auch: Cedro vermelho-rothe Ceder und Acaiaca. Gummi officinell. 
Acaia-catinga. Drepanospermum gummiferum Benth. Anacardiaceae. Liefert Gummi. Bauholz. Rinde riecht unangenehm, wird arzneilich benutzt. Liefert Harz.

Icacumeri. Erythrina glauca Willd. Papilionaceae. Heisst auch : Arvoeiro. Bauholz.

I cajarana. Lophophytum mirabile Schott. Balanophorae. Heisst auch: Fel de terra. Arzneilich benutzt, enthält Lophophytin.

Acajú cica. Gummi von Anacardium occidentale L. Heisst auch: Acayú-yeyca, Gonma de cajú. Wird arzneilich benutzt, sowie von den Buchbindern als Kleister: wird dann nicht von Insekten beschädigt.

Acajú. Anacardium occidentale L. Anacardiaceae. Heisst auch: Acaja-iba, Acajú-iba und Cajú. Früchte wohlschmeckend, Rinde arzneilich benutzt.

Acajú-y. Anacardinm hnmile Mart. Anacardiaceae. Früchte essbar, Rinde als Specificum bei Diabetes.

A cambuy. Engenia Telloziana Bg. Myrtacex. Frichte essbar.

Blätter und Rinde adstringens.

Acajurana und Acajurama. Acajurana pulchra Fr. Allem.

Leguminosix. Die ekelerregend riechende, bitter schmeckende Rinde Tolksmittel.

Ica parajúba. Hortia coccinea Spruce. Rutacex. Rinde officinell. Acapociba. Allemanda Schottii. Pohl. Apocynacex. Blätter und Wurzel.

A с a ṕra. Tupibenennung des hiesigen Flieders. Sambucus australis Cham. Caprifoliacer. Blätter als Abführmittel. Rinde als tonicum.

A сари́. Andira Aubletii Benth. Papilionacex. Rinde adstringens.

Bauholz. Spec. Gew. 1.112.

A ca pú rajado. Gemaserter A. Andira nitida Mart. Papilionacer. Möbelholz.

A capu-rana. Tupi-von acapoc-Baum mit aufspringender Frucht und rana - falsch, also Falscher Springfruchtbaum. Ticorea longiflora DC. Rutacex. Bittere Rinde. Bauholz. Spec. Gew. 1.105 .

Acarajé. Vide die Speise Abará wenn in Dendeöl gebraten. A carú. Vide Speise Abará, wenn ohne Capsicum bereitet.

A cara-úra. Baum im Norden, dessen Zweige zu Angelruthen, besonders zum Fange des Fisches Cará. (Acara nassa Heckel). 
Ararizoba. Hyrrorotyle nmbellata L. Var. honariensis Siprou...

l'mbellif rar. Ist officinell.

A cassa. Volksspeise im Staate Bahia, ron Reismehl, l'feffer etc.

A eataya. Spondias lutea L. var glabra Engl. Anararlialrar.

Friichte essbar. Wurzelknollen dienen zur Nahrur.g.

A caúan-caa. Mikania amaral Wille. Compositse. Bliitter leej

Schlangenbiss.

A catuaba. Anemopaegma mirandum A. I)C. Var. verticillata Bur.

Bignonialces. Wurzel als aphrodosiacum.

Acaya. Spondias purpurea L. var. venulosa Mart. Anacardiarear.

Frucht wohlschmeckend.

Acaya-assú. Grosse Cajá. Syomelias macrocarpal Engl. Anacar-

diacese. Frïchte essbar.

A ca ya-merim. Kleine Cajá vicle Leataya.

Accende candeia-Lampenanzïnder. Cassial biffora L. Carsal-

pineale. Brennt sehr gut. Stamm zu Mïbeln.

Aceitona. Brasil. Olive. Vitex Montevidensis Cham. Verbenacear.

Frucht essbar.

Aceitona do mato. Wilde Olive. Myrsine Hocrulosa Mart. Myzsineale. Frucht wird von den Indianern genossen.

Acelga. Weisser Mangold. Betal ricla L. Chenopoliaceae. Bläter als Gemüisse.

Achi. Vide Axi.

Achirá-mourou. Cordia nodosa Lam. Cordiaceae. Früchte ess-

bar. Rinde adstringens.

Achit. Vitis salutaris Bak. Ampelidacene. Wurzel und Blätter oxicinell.

A chite. Vide Caatigua, Catigôa.

Acroatá. Baum, von dessen Zweigen der Indianerstamm Guaycurís

Zäume für Pferde anfertigt.

A cumão. Cocos campestris Mart. Palmae.

Acum $\tilde{\pi}$ o rasteira. Kriechender A. Cocos petraea Mart. Palmae.

Acuna. Iriartea exorrhiza var. Orbigiana Mart. Palmae.

A curi. Attalea phalerata Mart. Palmae.

A derno. Astroninu fraxinifolinm Schott. Anacardiaceae. Vorzügliches Bauholz. Spec. Gew. 0.876. Frïchte liefern Oel, der Stamm Gummi.

A fiou. Arracacha esculenta D. C. Umbelliferae. Knollen als Nahrung. $\Lambda$ gapú. Vide Acapú. 
Agoniada. Plnmeria lancifolia Mart. Apocynaceale. Rinde emmenagogum und antifebril, enthält kryst. Glucosid-Agoniadin.

Agonty-treva. Anona vepertonum Mart. Anonaceae. Frïchte und Samen benutzt.

Agrião. Nasturtium officinale R. Br. Cruciferae. Heilmittel und zu Salat.

Agrião de Pará. Spilanthus acmella L. Compositae. Officinell. Agontiguepé. Die Tupibenennung für Araruta.

Agrimonda. Argemone mexican» L. Papaveraceae. Samen und Blätter officinell.

Agrimonia. Agrimonia parviflora Art. Rosaceae. Pflanze officinell. Aguai. Balsam von Myrocarpus fastigiatus Fr. Allem. vide Cabure-iba.

Aguapé. Nymphaea ampla I)C. var. speciosa Casp. Nymphaeaceae. Blätter Heilmittel.

Aguapé. Eichhornia aznrea Kth. var. rhizantea. Pontederiaceae. A gua pé. Pontelderia cordifolia Mart. Pontederiaceae.

Aguapé branco. Weisse A. Nymphaea Gardneriana Blanck.

Nymphaeaceae.

Aguapé cheiroso. Wohlriechende A. vide Aguapé branco.

Aguará-cuinha. Maloustia virescens R. Spruc. Apocynaceae.

Rinde und Milch-toxisch.

Aguará-cuinha assú. Grosse A. Heliophytum elongatum DC.

und inflicum DC. Heliotropieae. Blätter officinell.

Aguará-ponda. Tupiname für Gervao. Stachytarpha dichotoma

Vahl Verbenaceae. Blätter officinell.

Aguará-quiya. Solanum nigrum L. var. aguará quiya Piso.

Solanaceae. Officinell.

Aguará-quiya-assú. Grosse A. Solanum nigrum L. var. angulosum Sendt. Solanaceae. Officinell.

Aguará-yba. Fuchsfruchtbaum. Schinus terebinthifolius Radd.

Anacarcliacear. Beeren und Blä ter als Heilmittel.

Aguará-yba assú. Schinus molle L. Anacmrliaceae.

Aguaribay. Croton asperrimus Benth. Enphorbiaceae. Volksmittel.

Aguaxima. Pothomorphe sislaefolia Meg. Piperaceae. Blätter und Wurzel officinell.

Agulha do mato. Clitoria stipularis Benth. Papilionaceae. Volksmittel. 
Agutiguepo-obi. Thalia geniculata L. Marantareile. Bliitter Heilmittel, Wurzel essbar.

Aguti-namy. Cephäilis tomentosal Willd. Rubiaceae. Wurzel als Heilmittel.

Aguti-tiriba. Lncuma Rivicôa Gärtn. Sípotaceate. Frïchte essbar. Rinde offlcinell.

A houai. Thevetia Ahonai A. I)C. Aporenaceate. Milch, Blätter und Samen toxisch. Thevetin.

Aipim. Manilhot palmata var. aipi Mill. Arg. Euporbiacease. Wurzelriibe zur Nalırung.

А iri. Virle Brjaúbá.

A iúba. Aydendron permolle. Nees. Lannineize. Rinde Volksmittel. Bauholz.

A jarana. Trichilia sp. Meliaceae. Vorziigliches Bauhol\%.

A jaré. Tephrosia nitens Benth. Papilionaceale. Fischbetiiubungsmittel.

A jeurana. Hirtella Martiana Hook. Fil. Rosaceate. Friichte essbar.

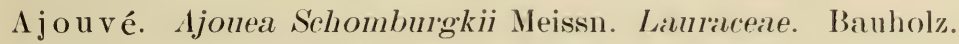

$\Lambda \mathrm{juba.} \mathrm{Ajonea} \mathrm{tenella} \mathrm{Nees.} \mathrm{Lanraceae.} \mathrm{Bauholz.}$

Ajuba-pita. Vide Jabotapita.

Ajurú. Von uá Frucht und urú Papagei. Chrysolualanus Ieraco I. Chrysobalanaceae. Rinde zum Färben. Frucht wird von den Indianern genossen.

Alandra. Nerium oleander L. Apocynaceale, kultivirt.

Alleará. Canna denudata Rosc. Cannaceae. Wurzel Volksmittel. Samen dient den Indianer zum Halsbandschmuck.

Alcamphoreira. Croton antisyphiliticum Mill Arg. Wurzel camphorartig riechend, Heilmittel bei Syphilis.

A l caparra. Kapern. Capparis spino:a L. Capparideae, kultivirt.

Alcassus bravo. Wilder Süssholz. Cassia rugosa Don. Caesalpinieae. Wurzel officinell.

Alcassus da terra. Einheimisches Süssholz. Teriandra dulois Mart. Caesalpinieae. Vollkommener Ersatz der Rad. glv erizae, enth. Glycericin.

Alcornoco. Bowdichia virgiloides HB. Kth. Caesalpinieae. Rinde officinell.

Aldaró. Indianische Benennung der Milho de angola.

Alecrim bravo. Wilder Rosmarin. Hypericum laxiusculum St. Hil. Hypericaceae Arom. Blätter officinell. 
Alecrim do campo. Prärierosmarin. Lantana macroplrylla

Schauer. Verbenaceae. Aromat. Pflanze Volksmittel.

Alecrim do mato. Waldrosmarin. Mikania sessilifolia DC. und Eupatorinm monardifolinm Walp. Compositae. Blätter Heilmittel. Alecrim das paredes. Kletternder Rosmarin. Chuquiraga floribunda Bak. Compositae. Aromatische Pflanze als Heilmittel. Alecrim da praia. Küstenrosmarin. Oncostylis arenaria N. ab.

E. Cyperaceae; im Staate S. Catharina Polygala adenophylla St. Hil. Polygaleae Von beiden die Wurzel.

Alecrim de S. Jose. Heiliger Josephsrosmarin. Portulacca pilosa

L. Portulaccaceae. Das bliihende Kraut als Heilmittel.

Alecrim selvagem. Wilder R. Baccharis $c$ issinaefolia DC. Compositre. Arom. Pflanze officinell.

Alecrim da serra. Gebirgsrosmarin. Rhytiglossa lencophlora N. ab. E. Acanthaceae. Aromat. Pflanze. Volksheilmittel.

Alecrim dos taboleiros. Hochebenen R. vide Alecrim da serra. Alegria. Himeranthus runcinatus Endl. Solanaceae.

Alevante. Peltodon radicans Pohl. Labiatae. Arom. Pflanze Heilmittel.

Alface dos rios. Flusssalat. Simolus valerandi L. Primulaceate. Volksmittel.

Alfacina. Kleiner Salat, vide Alface dos rios.

Alfafa brava. Wilde Luzerne. Desmodium barhatum Benth. Papilionaceae. Ein Lieblingsfressen der P.erde, Esel etc.

Alfavaca. Basilienkraut. Ocimum gratissimun L. Labiatae.

Arom. Pflanze. Officinell.

Alfavaca brava. Willes B. Hyptis fruticosa Salzm. Labiatae. Arom. Pflanze.

Alfavaca cabelluda. Harriges B. Hyptis pectinata Port. Labiatae. Aromat. Heilmittel.

Alfavaca do campo. Prärie R. Ocimum micranthum Labiatae Willd. Arom. Mittel.

Ilfavaca de cheiro. Wohlriechendes B. Ocimum basilicum L. vir. fluminense Vellos Labiatae. Officinell.

A lfavaca de cobra. Schlangen B. Monnieria trifolia L. Rutaceae. Schwitzmittel; im Staate Rio Grande do sul Steplianophysum angustiflorum Nahc. Acanthaceae.

Alfavaca sylvestre. Wald B. Ocimum nudicaule Benth. Labiatae. Arom. Pflanze. 
Alfazema de caboclo. Indianer Lavendel. Salvial rigilla Benth. Labialtae.

Alfazema do mato. Wald Lavindel. Keithia villosa Benth. var. glabriuscula Schmidt. Labiatae. Arom. Heilmittel.

Alfinete do campo. Prärienadel. Hyptis latiflora Mart. Labliatiae. Arom. Pflanze.

Algodoeira. Baumwollbaum. Gossypinm barbatum L. Malvaceae. Algodoeira bravo. Wilder B. Gossypinm religiosum L. Malvaceae.

Algodoeira do campo. Prärie B. Cochlospermum insigne St. Hil. Bixaceae. Samen zur Oelbereitung. Wurzel statt Rhabarber. Algodoeira felpuda. Kottiger B. Gossypinm hirsutum Sw. Malvaceae.

Algodøira do mato. Wald B. Bombax ceiba L. Bombiacers. Gelbe Samenwolle. Samen ölreich.

Algodaosinho. Kleiner B. Ipomara fistulosa Mart. Convolvulacean. Sparsame gelbe Pflanzenwolle. Wurzel ofticinell. Blätter schäilich für Ziegen.

Alho do mato. Waldknoblauch. ('ipural palurlosal Aubl. Iridlaceate. Wurzel abfïhrend.

Aliculi. Cocos schyzophylli Mart. Palmine.

A licuri vide Aliculi.

Alleluia. Eupatorium sordescens DC. Compositae. Bliitter off. $\Lambda 1 \mathrm{meceg}$ aо. Almecegueirahar $=$ Elemi.

Almecegueira. Elemibaum. Protinm icacariba March. Burseraces. Im Staate Bahia ist $P$. aromaticnm Engl; im Staate Minas I'. Warmingianum March; im Staate Pará $P$. altissinnum March, und im Staate Rio Grande P. ovatum Engl. Sämmtliche liefern Elemi.

Almecegueira brava. Wilder E. Icicopsis brasiliensis Endl. Burseraceae. Liefert ebenfalls Elemi.

Almecegueira do campo. Prärie E. Proteum almecega March. Bur:eraceae. Liefert sehr reichlich Harz.

Almecegueira da serra. Gebirgs E. Proteum brasiliense Engl. Almecegueira ver in el ha. Rother E. Proteum heptophyllum March. var. brasiliensis Engl. Burseraceae. Liefert dickflüssiges Harz. Althea da terra. Einheimische A. Sida cordifolia L. Malvaceae. Wurzel.

Alumão. Nicht bestimmte Pflanze in Bahia. Volksmittel. 
Alvacana. Nicht bestimmte Pflanze. Volksmittel in den Nordstaaten.

Amaitin. Pourouma bicolor Mart. Urticaceae. Frucht essbar. Bauholz.

A majoura. Ajouea brasiliensis Meissn. Lauraceae. Blätter. Holz zu Bauten.

A mandoi-rana und $\mathrm{A}$ mandurana vide Algodoeira brava.

A maniú vide Algodoeira.

Amanôa. Amanoa guianensis Aubl. var. genuina Müll. Arg. Euphorbiaceae. Bauholz.

Amansa besta. Maulthierzähmer. Oreodaphne Martiana Rich. Lauraceae. Ruthen für Eseltreiber.

A mapá. Plumeria fallix Müll. Arg. Apocynaceae. Blätter und bittere Rinde; Milch toxisch wirkend. Bauholz.

A ma paima. Tupiname für Casca preciosa.

A marante. Crudya amazonica Spruc. Caesalpiniae. Samen ofticinell. Bauholz. Spec. Gew. 0.967.

A maré. Metrodorea excelsa Fr. Allem. Rutaceae. Rinde antifebril. Bauholz.

A marellinho. Galipea simplicifolia Engl. Rutaceae. Rinde off. Möbelholz.

A marello. Connarus favosus Planch. Connarazea. Vorzïgliches Bauholz.

A marello flor de algedao. Connarus fulvus P'lanch. Connaraceae.

Schönes gelbes Möbelholz.

A mari vide Amarello.

A m baia vide Ambaiba.

A mbaia-embo. Aristolochia labiosa Ker. Aristolochiaceae. Bei Schlangenbiss.

A mbaia-tinga. Weisser Trompetenbaum. Cecropia palmata Willd.

Crticareae.

A m baiba. Trompetenbaum. Cecropia adenocarpus Mart. Uríicaceae. A m baiba branca vide Ambaia-tinga.

A m baiba prateada. Silber A. Cecropia hololenca Miq. Lrticaceae. A mba-paya. Tupiname für Carica papaya $L$.

A m bar vegetal. Vegetabilischer Ambra. Hedyosmum brasiliense

- Mart. Chloranthaceae. Blätter riechen ambraartig.

A mbauva brava. Wilder $\Lambda$. vide Amaitin.

Ambauva mansa. Zahmer A. Pourouma cecropiaefolia Mart. Urticaceae. Frucht wohlschmeckend. 
A mbauva de vinho. Wein A. Poucronma tomensosa Mart.

Urticaceae. Frucht essbar, dient zur Bereitung eines Getränkes. Ambauva do mato. Wald A. Pouronma acmminata Mart.

Urticaceae. Die wenig schmackhafte Frucht dient den Indianern zur Nahrung.

A mbé. Aracaceae. Existirt am Amazonenstro n; dessen Luftwurzeln zum Binden der Sarsaparillbiindel benutzt.

A mbelania. Ambelania acida Aubl. Apocynaceae. Milch u. Rinde. Ambrosia. Ambrosia polystachya DC. Compositae. Blättel off. A m bira vide Pindahyba.

A m bú vide Acataya.

A m bú-y. Tupiname vide Ameixeira da terra.

A m burana vicle Imburana.

Ambura-rembo. Tupiname vide Papo de perír.

Ameixeira de Canada vide Ameixeira da India.

Ameixeira de espinho. Stachelpflaumenbaum. Ximenia coriacea Engl. Olacineae. Frucht essbar.

A meixeira da India. Indischer Pflaumenbaum. Erybotvra japonica Lindl. Pomaceas. Frucht wohlschmeckend. Blitithen und Samen blausäurehaltig.

A meixeira da terra. Einheimischer-Pfl. Timenia americana L. Olacineae. Frucht und Samen essbar.

A meixeira preta. Schwarzer Pfl. Prunus paranaensis Lacerd. Amygdaleae. Frucht essbar.

A mend oeir a brava. Wilder Mandelbaum. Cerasus brasliensis Cham. et Schlecht. Amygdaleae. Rinde und Samen blausäurehaltig.

Amendoim. Erdnuss. Arachis hypogaea L. Papilionaceae. Samen und Oel.

Amendoim falso. Falsche Erdnuss. Vide Mandubira'la.

A mendoim mandiocca. Mandiocea-Erdnuss. Arachis tuberosa

Bong. Papilionaceae. Samen und Wurzelknollen genossen.

Amonjeaba. Panicum Myuros Lam. Gramineae. Emolliens.

Amor do campo. Zornia brasiliensis Vog. Papilionaceae. Blätter officinell.

Amor crescido. Portulacca grandiflora Hook. Portulaccaceae. Zierpflanze. Saft bei Erysipelas.

A mor dos homens. Männerliebe. Hibiscus mutabilis I. Malvaceae.

Zierpflanze. Die Benennung wegen der Veränderlichkeit der Blïthenfarben. 
Amor de negro. Negerliebe. Vide Carrapicho do campo.

Amoreira do mato. Wilder Maulbeerbaum. Brosimum Gandichaudii Trec. Urticaceae. Frucht essbar. Bastpflanze.

Amora preta. Schwarze Brombeere. Rubus urticaefolius Poir. Rosaceae. Frucht wohlschmeckend.

Amora da silva. Waldbrombeere. Rubus brasiliensis Mart. Rubiaceae. Frucht essbar.

Amora verde. Grïne Brombeere. Rubus erythroclardos Mart.

Rosaceae. Die in reifem Zustande grïne Frucht eine Delikatesse. A mores. Viele Liebe. Aeschynomene sensitiva Sw. Papilionaceae. Blätter Aphrodisiacum.

A mostrinho. Gestossener Kautabak zum Schnupfen.

Anabi. Potalia amara Aubl. Loganiaceae. Blätter und bittere Rinde off.

Anacôco. Swartzia tomentosa DC. Papilionaceae. Rinde sudorif. Bauholz.

A najá. Maximiliana regia Mart. Palmae. Der Tupiname bedeutet = essbare Frucht.

Anája. Pinclarea concinna Barb. Rodr. Palmae.

Anája-naya. Pindarea fastuosa Barb. Rodr. Palmae.

Anajá-merim. Kleine A. Attalea humilis Mart. Palmae.

Anana. Guaranibenennung für Ananaz.

Anana\%. Ananas sativus Lindl. Bromeliaceae. Bekannt.

$\Lambda$ nanaz de agulha. Nadel A. zufolge der spitzstacheligen Blätter.

Ananas muricatus Schult. Bromeliaceae. Frucht essbar. Blätter zu Faser.

A nanaz bravo. Wilde A. Ananas sylvestris Schult. Bromeliaceae. Blätter vorzïgliche Faser. Früchte als emenagogum und abortivum.

Ananim vide Oanani.

Anany. Hevea panciflora Müll. Arg. Euphorbiaceae. Bauholz, liefert Cautchouc.

Anario. Ipomea polyrhizos Chois. Convolvulaceae. Die geröstete Knolle Nahrung der Indianer.

Andá-assú. Grosser Andahaum. Johannesia princeps Vellos. Euphorbiaceae. Samen und Wurzelknollen drasticum.

Andaca vide Trepoeraba.

Anday a-assú. Grosser A. Attalea indiava Dr. Palmae. Frucht essbar. 
Andiroba. Carapar guyanemsis Aubl. Mrliafear. Cormmpirtes Tupiwort von landi-Oel, roba=bitter. Bittere Rinde, Samen und Oel off. Bauholz.

Andiroba vide Jabotá.

A nolirova vide Fava s. Ignacio.

Andirá. Tupibenennung fiir Angelim amalogosa, heisst Flederman.

Andirá. Ibiaiariba. Rothe $\Lambda$. Virle Angelim doce und Iba-iariba.

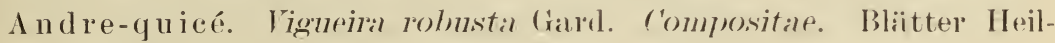
mittel.

A nd ú vide Guandú.

Andurá-obaja. Tupiname fuir Angelica.

Anga-iba-tam vide Craveiro do mato.

Anga-iba-atan. Cor lial nmbrarnlitirar DC: Corrliarrar. Bauholz: atan und antam bedeutet festes Hol\%.

Angelica. Guettarela angelifal Mart. Rulivarate. Scharf arom. Wurzel off.

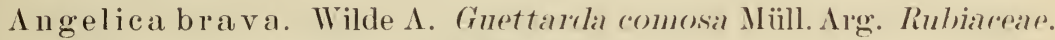

Bittere, toxisch wirkende Wurzel.

Angelica do mato. Wald A. Lisianthus pernnculatus Schl.

Gentianear. Die bittere Wurzel als Ersatz der Enzianwurzel.

Angelica de Parà. Dicorynial paralïnsis Benth. Casalpiniale.

Bittere Rinde. Bauholz. Spec. Gew. (0.857.

Angelica de ramos. Bouquett A. Srhnloertia multiflor: Mart.

Asclepiaclaceae. Zierpflanze. Blaitter als aromat. u. excitans.

Milch zum Vergiften d. Thiere.

Angelicó. Aristolorhia tribolata L. Aristolochiaceae. Antidot bei Schlangenbiss.

Angelim. Tipnana heteropteral Benth. Papilionaceas. Bauholz.

Spec. Gew. 1.110 .

Angelim amargoso. Bittere A. Andira vermifuga Mart. Papilionaceae. Die bitteren Samen Wurmmittel, enthalten Andirin.

Bauholz.

Angelim de folha larga. Breitblätrige A. Andira anthelmintica

Benth. Papilionaceae. Bittere, andirinhaltige Samen vorzügliches

Wurmmittel. Bauholz. Spec. Gew. 1.007.

Angelim branco. Weisse A. Pterogyne nitens Tulasne. Papilionaceae. Bauholz.

Angelim côco. Nuss A. Andira stipulacea Benth. Papilionaceate. Bauholz. Samen anthelminticum. 
Angelim doce. Süsser A. Andira fraxinifolia Benth. Papilionaceae. Samen drasticum. Bauholz.

Angelim pedra. Stein A. Ferreira spertabilis Fr. Allem. Papilionaceas. Steinhartes Holz, deshalb der Name; im Splint der alten

Bäume in Menge ein Thonerde ähnliches Alkaloid Angelin (Ratanhin?).

Angelim rosa. Rosarothes A. Peraltea erythrinaefolia Sald.

Papilionaceae. Das rosarothe Holz zu Möbeln. Spec. Gew. 0.66:3.

Samen werden von den Indianern genossen.

Anga-yba-at: $n$ vide Canella brava.

Angelim tinto. Rothe A. Andira paniculata Benth. Papilionaceae.

Bauholz.

Ingelim da varzea. Wiesen A. Vide Angelim doce.

Angico. Piptadenia rigida Benth. Mimosae. Vorzügliches Bauholz.

Sp. Gew. 1.063. Rinde bitter, adstringirend. Reichlich Gummi

liefernd, officinell als Hustenmittel.

Angico branco. Weisse A. Piptadenia foliosa Benth. Mimosae.

Rinde zum Gerben. Bauholz.

Angico grande. Grosse A. Vide Angico de moenda.

A ngico miudo. Kleine A. Piptadenia contorta Benth. Mimosar.

Bauholz.

Angico de inoenda. Walzen A. Piptadenia colubrina Benth.

Mimosae. Vorzügliches Holz zur Anfertigung der Zuckerrohrwalzen und Möbelholz.

Angicovermelho. RotheA. Piptadenia inaequalis Benth. Mimosae.

Bauholz. Rinde adstringens.

Angiroba vide Andiroba (Carapa).

Angostura. Hortia brasiliana Vand. Rutaceae. Arom. bittere

Rinde off.

Anguay. Tupiname für Balsamo.

Anguhy-batan vide Craveiro do mato.

A nhangapá vide Aninga-pari.

Anhanga-K ybabb. Tupiname fïr Pente de macaco.

A nhanga-piri vide Aninga-pari.

Anhanga-puturú. Aristolochia theriaca Mart. Aristolochiaceae.

Wurzel Antidot bei Schlangenbiss.

Anhanga-quiabo vide Pente de macaco.

An hang-recuiba vine Caa-opia.

Anhauina. Ajouea densiflora Nees. Lanraceap. Bauholz. 
An hoaiba vicle Canella.

A n huiba vide Sassafraz.

Anhuiba mirim vide Canella mirim.

$\Lambda \mathrm{nhuiba-pé-ayba} \mathrm{vide} \mathrm{Canella} \mathrm{funcho.}$

Anhui-pitanga. Rothe $\Lambda$. Linhareal aromintical Irrnd. Lantraterar.

Aromat. Bl. u. Rinde off.

An ic a vide Caperiçoba.

A nil. Indigofera anil L. Papilionaceac. Blätter zur Bereitung des

Indigo. Wurzel emenagoguin.

Anil assú. Grosser Indigo. Enpatorinm pictum Gard. C'ompositae.

Zum Färben baumwollener Zeuge. Antidot bei Schlangenbiss.

Anil in Bahia. Intigoferar Blanchettiana Benth. Papilionaceate. Liefert Indigo.

Anil in Minas. Indigofera lespetezoifles HBKth. Papilionareate.

Liefert Indigo.

Anil in Parà. Indigofera pilscuormm Benth. Papilionaceate. Liefert Indigo.

Anil in Paraná. Intligofera asperifolia Bong. Papilionaceae. Liefert Indigo.

Anil in Pernambuco. Indigofera hirsuta L. Papilionaceae. Liefert Incligo.

Anil in Rio de Janeiro. Intigofera sabulicula Benth. Papilionaceate. Liefert Indigo.

Anil in Santa Catharina. Indigofera punicea Benth. Papilionacease. Liefert Indigo.

Anil in S. Paulo. Indigofera grarilis Bong. Papilionareate. Liefert Indigo.

Anil bravo. Wilder Indigo. Tephrosia toxicaria Pers. Papilionaceae. Blattzweige zum Betäuben der Fische; hat keinen Farbstoff. Anil do brejo. Sumpf Indigo. Aeschynomene brasiliana DC. Papilionaceae. Aphrodisiacum; so genannt zufolge der Blattähnlichkeit.

Anil do mato. Waldindigo. Eupatorinm laeve Dr. Compositae. Zum Färben baumwollener Zeuge.

Anil miudo. Kleiner Indigo. Indigofera microcarpa Desv. Papilionaceae. Liefert Indigo. Wurzel als desobstruens.

Anil dos pobres. Indigo der Armen. Poiretia angustifolia Vog. Papilionaceae. Blätter zum Färben baumwollener Zeuge und als Antidot bei Schlangenbiss. 
Anil trepador. Kletternder Indigo. Vitis sicyoides Bak. Ampelideae. Wurzelknollen Heilmittel. Blätter zum Schwarzfärben baumwollener Zeuge.

Anima-membeca. Maranta grandifolia Lindl. Marantaceae. Zierpflanze.

Aninga. Montrichardia linifera Schott. Araceae. Saft der Blätter Wundmittel.

Aninga d'agua. Wasserlinse. Caladium sororum Schott. Blätter Wundmittel. Die gerösteten Samen dienen den Indianern zur Nahrung.

A ninga-iba vide Aninga.

A ninga-pari. Melastoma paradoxa Mart. Melastomaceae. Blattpulver als Wundmittel.

A ninga-piri. Melastoma roccinea Vell. Melastomaceae. Blätter und Rinde off.

A ninga-uva vide Imbé da praia.

Anna Pinta vide Tayuya und Purga de caboclo.

Annuiba de brejo vide Canel'a branca de brejo.

Antenilha vide Pau ferro.

Annui-üva. Xylosma digynum Benth. Bixaceae. Bauholz.

A ouassi. Indianische Benennung fïr Zea Maïs.

Apanha sai:. Kleiderfänger. Ionidium atropnrpureum St. Hil.

Violaceae. Blätter haften klettenartig an den Kleidern. Wurzel officinell, enthält $0.040 \%$ Ionidin kryst.

A pacaiba und Apareiba vide Mangue vermelho.

Apaseira. Eperua falcata Aubl. Caesalpinica?. Vorzügliches Holz. A pé. Urospatha caudata Schott. Aracea . Der ätzende Wurzelsaft wird von den Indianern benutzt. Die gerösteten Knollen als Nahrung. A pé assú vide Mamminho de cachorro.

A péay. Salvinia auriculata Aubl. Salviniaceas. Pfanze Volksmittel. A pé-iba vide Jangadeira.

Aperta. Symplocos tetrandra Mart. Symplocaceae. Rinde adstringens.

A perta ruao. Artanthe Olfersiana M q. Piporaceac. Blätter und Zweige Heilmittel.

A perú vide Abajerú.

A pia-aconocú. Tupiname für Pau de lagarto.

A pihi. Dorstenia rotundifolia Arrud. Urticaceae. Wurzel officinell. A pióba. Guarea Sprucei C. DC. Meliaceae. Rinde arzneilich. 
A piú vide Apúy.

Apiy. Urostigma Gardnerianum Miq. Urticacease. Blätter und Milchsaft.

A pocuita-goará vide Apogita-goará.

A pogita-goará. Esenluerkia intermerlia Mart. Rutaceap. Rinde antifebril.

A postemeira. Geschwürsöifner. T'urnera molochioides Camb. var.

latifolia Urban. T'nrnerarease. Blattumschlag fiir Furunkel.

A poti-coraca. Enphorbia brasiliensis HBKth. var. pulchella Bois.

Euphorbiaceac. Blätter als Augenwasser; ferner gleiche Benennung

Bernardia sirlioirles Müill. Arg. Enporliareae. Bei Husten.

A prai-ú. Tupiname für Massaranduba von C'earà.

A puy. Clusia insignis Mart. Guttiferae. Milchsaft als Ersatz von Gummi gutta.

A ra. Indianische Benennung fïr Tinhor: $\widetilde{0}$.

Arabutan. Guaranibenennung fïr Paú Brasil.

Araça. Kultivirte A. Psidium araça Rard. Myrtaceae. Frucht essbar.

Araça in Pará. Britoa acicla Bg. Myrtaceale. Frucht essbar.

A raça in Pernambuc). Campomanesia pubescens Bg. Myrtaceate. Frucht essbar.

Araça in Rio Grande do Sul. Psillum grandifolium Bg. Myrtaceale. Frucht essbar.

Araça in S. Paulo. Vide Araça branea.

Araça branca. Weisse A. Psidium multiflorum Camb. Myrtaceae. Frucht essbar.

Araça bravo. Wilde A. Psidium cuneatum Bğ. und Psidium coriaceum Bg. Myrtaceae. Frucht essbar.

Araça de brejo. Sumpf A. Posoqueira leucantha Barb. Rodr. Rubiaceae. Frucht essbar.

Araço cagão. Psidium rufum Mart. Myrtaceae. Frïchte verursachen Durchfall.

Araça do campo. Prärie A. Campomanesia mediterranea Bg. Myrtaceae. Frucht essbar.

A raça de catingas. Gebüsch A. Psidium hians Mart. Myrtaceac. Herbe Frucht.

A ra ça de cheiro. Wohlriechende A., heisst auch Araça de congonlia. Campomanesia suaveolens Bg. Mrrtaceae. Blüthen wohlriechend, Frucht essbar. 
Araça de corôa. Kronen A. Psidium variabile Bg. Myrtaceae. Sehr wohlschmeckend.

Araça felpudo. Filzige A. Psidium incanescens Mart. Myrtaceae. Nicht wohlschmeckend.

Araça garamurú. Psidium Donianum Bg. Myrtaceae. Frucht wohlschmeckend.

Araça goiaba. Psidium Sellowianum Bg. Myrtaceae. Sehr wohlschnieckend.

Araça guassú vide Goiaba.

Araça lisà. Glatte A. Varietät von Psidium araça R. Frucht wohlschmeckend.

Araça do mato. Die wilde Psidium arara Radd. Früchte herbsüsslich; heisst auch :

Araça mirim. Kleine $A$.

A raça pedra. Psidium microcarpum Camb. Myrtaceae. Frucht essbar.

Araça pera. Birnen A. Die vorzïglichste und grösste Varietät ron Psidium araca Radd.

Araça piroca. Psidium riparium Mart. Myrtaceae. Adstringens.

Araça da praia. Psidium littorale Radd. Myrtaceae. Frucht essbar.

Araça-rama. Unbestimmt. Die Wurzeln dieser am Amazonenstrome vorkommenden Pflanze dienen den Schildkröten als Nahrung.

Araça roxa. Violetrothe A. Varietät von Psidium araça R. Frucht sehr wohlschmeckend.

Araça de tinguijar. Fischtötende A. Diospyros brasiliensis Mart. Ebenaceae. Die Zweige zum Betäuben der Fische.

Araça uarina. Psidium albidum Camb. Myrtaceae. Frucht wohlschmeckend.

Araça vermelha. Rothe A. vide Araça de corôa.

Araçahi. Campomanesia fı uticosa Bg. Mortaceae. Kleine Frucht essbar.

Araça uba. Psidium acutangulum DC. Mrrtaceae. Falsche Goiaba, essbar.

Araça zinho vide Arara mirim.

Araçazinho do mato. Wilde kleine Arara. Daria paniculata DC. Melastomaceae. Früchte essbar.

Aracouchin. Protium aracouchini Marsh. Burseraceae. Hurz. 
A racui. Tupiname für Angelim amargoso de folha larga.

A racuri und Aracuy vide Aliculi.

A rapabaca. Spigelia anthelmia L. Loganiacerse. Anthelminticum. $\Lambda$ rapaca brava. Wilde $\Lambda$. Spigelia glabrata Mart. Loganiareare.

Anthelmintisch.

A rapari. Macrolobium multijugum Benth. Caesalpiniae. Holz.

A rapiraca. Piptadenia macrorarpa Benth. Mimoseae. Bauholz. A rápoca. Raputia alba Nees et Mart. Rutareae. Rinde off. Bauholz. A rápoca a ma rella. Gelbe $\Lambda$. Galipaea dichotoma Fr. All. Rn-

tareae. Rinde und Bauholz. Spec. Gew. 1.021.

Arápoca assú. Grosse A. Raputia magnifica Engl. Rutaceae. Schiffsbauholz.

Arápoca branca. Weisse $\Lambda$. vide Arápoca und Candêa.

Arapoca miriun. Kleine $\Lambda$. Rania resinosa N. et Mart. Rutaceae.

Rinde und Holz.

A ra rà. Papagei. Indianische Benennumg für Amaranthus trirolor

L. Amaranthaceae.

A ra rabà vide Bugé.

A raranin vide Cutitiriba.

A ra rà-canga. Aspirlospermal conclvlorarpon Miill. Arg. Apoc:vnaceae. Holz und Rinde.

Arara-cueira. Aporynacene. Vorziigliches Bauholz.

A rar à-jua. Meliaceae. Bauholz.

A rarà-petui vicle Rabo de ararì.

Arar à-rambe. Baubolz.

A rarà-tucupi. Parkia oppositifolia Meissn. Mimoseae. Die Fruchtschote ein beliebtes Fressen des Ararápapagei.

A ra rà-iiva vide Arâryba le Minas.

Araribá. Sickingia rubra Schum. Rubiareae. Rinde antifebril, ent-

hält Araribin; schönes Möbel- und Bauholz, rosaroth.

Araribá a marella. Gelbe A. Sickingia Oliveri Schum. Rubiaceae.

Bauholz.

Araribá branca. Weisse A. Sickingia viridiflora Schum. Rubiaceae.

Weiches Holz.

Araribá piranga. Rothe A., und Araribá rosa, Rosa A., vide Araribá.

Araribá tinga vide Araribá branco.

Arariba vermelha. Rothe A. Sickingia Glasiovii Schum. Rubiaceae. Bauholz. 
Arâryba. Centrolobium robustum Mart. Papilionaceae. Vorzügliches Bauholz und Möbelholz.

A râryba do campo. Prärie A. Connarus suberosus Planch. Connaraceae. Bauholz.

Arâryba femea. Weibliche A. Dalbergia foiiosa Benth. Papilionaceae. Bauholz.

Arâryba macho. Männliche A. Machaerinm Gardner’j Benth. Papilionaceae. Bauholz.

A râry ba in Minas. Centrolobium tomentosum Benth. Papilionaceae.

Vorzügliches Bauholz. Von Arà - Papagei und yba - Holz.

Arâryba roxo. Violetrothe A. vide Arâryba.

Ararixa. Sterculea Chicha St. Hil. Sterculeaceae. Samen als Ersatz der Mandeln.

A rarixú vide Herva moura.

Araroba. Andira araroba Macedo. Papilionaceae. Araroba offic. Araruta. Maranta arundinacea L. Marantaceae. Arrow-root.

Arataia. Posqueria calantha Barb. Rodr. Rubiaceae. Rinde und Frucht.

Araticum. Rollinia exalbida Mart. Anonaceae. Rinde, Bast und Gummi.

Araticum de alagadisso. Anona spinescens Mart. Anonaceae. Frucht und Bast.

Araticum-a pé. Anona pisonis Mart. Anonaceae. Frucht essbar. Iraticum de arêa. Anona Salzmannii A. DC. Anonaceae. Frucht, Bast und Holz.

Araticum assú. Anona dioica St. Hil. Anonaceae. Frucht und Samen.

Araticum do brejo. Sumpf A. Anona palustris L. Anonaceae.

Frucht schädlich, Wurzel als Korkersatz.

Araticum cagão. Durchfall A. Anona furfuracea St. Hil. Anonaceae. Frucht. Samenabsud gegen Ungeziefer. Bast und Holz. Araticum do campo. Prärie A. Anona coriacea Mart. Anonaceae. Frucht und Bast.

Araticum das Catingas
Araticum lisa

Araticum mangue. Mangle A. Anona rhizantha Eichl. Anonaceæ.

Rinde und Holz.

Araticum do mato. Wilde A. Rollinia silvatica Mart. Anonaceae.

Gummi und Holz.

Araticum panam vide Araticum do breyo.

Iraticum pitaya vide Atta. 
Araticum ponhé. Anona Marrgrarii Mart. Anonaceate. Frucht. Araticum puante. Stinkende 1 . Anomal foetirla Mart. Anomaceale. Bast.

Araticum do rio. Fluss $\Lambda$. Vide Araticum de alagadisso.

Araticum de Santa Catharina. Rollinial salicifolia Schlecht.

Anonacer. Gummi, Bast und Hol\%.

A raticú-titaya vide fructa de Condessa.

A raxina. Vochysia gum nifera Murt. Vochysiacese. Gummilieferant.

Bauholz.

A rarixú. Tupiname fïr Herva Moura.

Arco de pipa. Tonnenreifen. Ervthroxylum utilissimum Fr. Allem.

Erythroxylaces. Bauholz, Zweige zu Reifen.

A reo de pipa vide Subrasil.

Arco de pipa miudo. Kleine T. Erythoxylum fiangulaefolium

St. Hil. Erythoxylaceæ. Rinde zu Fassreifen.

A requim. Tupiname für Angelim pedra.

A rgueiro. Ormosia fistigiata Tul. Pappilionareate. Samen Schmuck

der Indianer. Bauholz.

A rica. Unhestimmt. Bauholz.

Aricanga. Geonoma Schottiana Mart. P'allire. Frucht.

A ricanga da terra. Geonoma erythrosparlian Barb. Rodrg. Palmip.

Aricuhy. Andira frondosa Mart. Papilionacese. Bauholz u. Blätter.

A ricuri vide Aliculi.

Aricury vide Uricuri-iba.

A rikuryoba. Arikuryobal Calpanemae Barb. Rodrig. Palmie.

A rimarú. Strychnos pedunculata Benth. Loganiaceae. Rinde und

Frucht.

Ariri vide Aliculi.

A rmim. Unbestimmt. Bauholz.

A rnica. Chionolaena latifolia Bak. Compositæ. I Blïthen dienen Solidago microglossa DC. Compositre. f dem Volke als Eupatorium conyzoides Vahl. Compositre. Ersatz d. Arnica.

A rnica do campo. Prärie A. Lavradia ericoides St. Hil. Sauvagesiaceae. Wundmittel Ersatz der Arnica.

A rnotte vide Urucú.

A roeira. Schinus molle L. var. Areira DC. Anacardiaceae. Blätter, Rinde und Holz.

Aroeira amarella. Gelbe A. Schinus dependens Orteg. Anacardiaceae. Bauholz. 
Aroeira branca. Weisse A. Shinus terebinthifolius Radd. var. acutifolia Engl. Allarardiaceaze. Rinde und Blätter aromatische Heilmittel.

Aroeira branca de capoeira. Weisse Gebüsch A. Lithrea molleoides Engl. Anacardiaceae. Frü̈clite und Blätter aromatisch. Rinde zum Färben.

Aroeira do campo ride Aroeira rasteira und auch Grundeura.

Aroeira do campo in Minas. Prärie A. Schinus terebinthifolius

Radd. rar. Sellowiana Engl. Anacardiaceae. Arom. Früchte u. Blätter.

Aroeira de capoeira. Gebüsch A. Schinus terebinthifolius Radd.

rar. Raddiana Engl. Anacardiaceae. Aromatische Früchte und Blätter. Bauholz.

Aroeira de Ceara ride Grundeura.

Aroeira do matto rirgem. Lrwald A. vide Aroeira preta.

Aroeira preta. Astronium fraxinifolium Schott. Anacardiaceae.

Vorzügliches Bau- und Möbelholz.

Aroeira da praia. Küsten A. Schinus lentiscifolius L. Marsh.

Anacardiaceae. Früchte, Blätter, Rinde und Harz.

Aroeira rajada. Gemaserte A. Schinus terebinthifolius Radd. rar.

Pohliana Engl. Anacardiaceae. Schönes Möbelholz. Früchte und Blätter arom.

Aroeira rasteira. Kriechende A. Shinus Weinnannifolius Mart.

Anacardiaceate. Früchte, Blätter und Rinde.

Aroeira da serra. Gebirgs A. Schinus terebinthifolius Radd. var.

Glasioriana Engl. Anacardiaceap. Frïchte. Blätter und Rinde. Aroeira vermelha. Rothe A. Schinus terebinthifolius Radd. var. rhoifolia Engl. Anacardiaceze. Blätter und Fiüchte harzreich und aromatisch. Bauhol\%.

Aroeirinha. Kleine A. vide Aroeira branca und Aroeira branca de capoeira.

Arourou. Protuim Schomburgkianum Engl. Burseraceae. Art Elemiharz liefernd.

Aroma. Acacia C'arenia Hook. et Arn. Minosale. Blïthen und Gummi.

Aroma ride Espongeiı.

Arra diabo. Schmerzausruf zufolge der giftigen stacheln. Jatropha urens rar. neglecta Mïill. Arg. und J. Oligandra Mï̈ll. Arg. Euphorbiaceae. Samen ölreich. 
Arrebenta. Aufplatzer. Solanum variabile Mart. Solanaleate. Früchte giftig.

Arrebenta boi. Ochsenaufplatzer. Solanum sodominm L. Solanaceae. Frichte giftig.

Arrebenta buxo. Magenplatzer. Unbestimmter Strauch auf der

Sträflingsinsel Fernan.lo de Noronha; der blawe Saft der Früichte dient den Sträflingen als Ersatz d r Tinte, wirkt toxisch.

Arrebenta cavallo. Pferdeaufplatzer. Solanum aculeatisiminm

Jacq. Solanaceae. Die unreifen Friichte reich an Solanin.

Arrependido. Kreuzdorn; man bereut es, wenn man denselben berührt. Gouania discolor Benth. Rhamneae. Die '/weige sehr flexibel, zum Flechten.

Aringa-iba und Arringa-iba. Philodendron speciosum Schott. Araceae. Blätter officinell. Wurzelknolle essbar.

Arriozes. Caesalpinia Bonducella Roxb. Caesalpiniae. Samen officinell.

Arroz. Reis. Oryza sativa L. Gramineae.

Arroz de cuxa. Nationalspeise im Staate Bahia von Hibiscus esculentus und sabdariffi, Sesamsamen und Reis.

Arroz do mato. Waldreis. Oryza subulata Nees ab E. Graminex. Arroz sylvestre. Wilder Reis. Luziola Peruviana Pers. Gramineae. Dient im Staate Rio Grande do Sul zur Nahrung.

Arruda. Raute. Ruta bracteosa DC. Rutaceae.

Arruda brava. Wilde Raute. Symphiopappus reticulatus Bak.

Compositae. Officinell.

Arruda de campina. Feldraute. Poiretia scandens Verb. Papilionaceae. Blätter off.

Arruda do campo. Prärieraute. Hypericum teretiusculum st. Hil. Hypericaceae. Starkriechende Pflanze als Heilmittel.

Arruda do mato. Waldraute im Staate Rio de Janeiro. Xanthoxylum Peckoltianum Engl. Rutareae. Starkriechende Blätter als emmenagogum etc. Im Staate Pernambuco ist es Pilocarpus officinalis Aubl. Rutaceae.

Artemisia da praia. Küsten Beifuss. Ambrosia humilis Vellos.

Compositae.

A rundey-pita vide Grundeuva.

Arupemba vide Urupemba.

Arvoeira. Mastenbaum. Erythrina glauca Willd. Papilionaceae Samen Heilmittel. Bauholz. 
Arvoeiro. Portug. Benennung fïr Jequirity.

Arrore de alho. Knoblauchbaum. Seguieria Langsdortfii Mag.

Phytolaccaceae. Blätter und Blüthen Heilmittel. Holz giebt kalireiche Asche.

Arvore de burro. Nicht bestimmt; eine giftige Euphorbiaceate der Insel Fernando de Noronha.

Arvore de cera. Wachsbaum. Myrical segregata Iacq. Myriaceae.

Pflanzeuwachs.

Arrore de chura. Regenbaum. Pithecolobiun samam Benth.

Mimoseae.

Arvore de lãa. Wollbaum, vide Barreguda.

Arvore de mammona vide Mammona do mato.

Arvore de manteiga. Butterbaum. Caryocar nuciferum L. Rihizoboleae. Samen und Fett.

Arvore de mosquitos. Mückenbaum. Escaecaria pallida Müll.

Arg. Euphorbiaceae. Blätter verursachen Entzündung der Haut. Baubolz.

Arvore de paina vide Paineira.

Arrore de pão. Brodfruchtbaum. Artocarpus incisa L. Lrticaceae. Arvore santa vide Cinnamomo.

Arvore de sebo. Talgbaum. Excaecaria sebifera Müll. Arg. Euphorbiaceale. Fett der Samen und Bauholz.

Arvore de sombra. Schattenbaum, vide Anga-iba-atan.

Arvore triste. Trauerbaum, vide Issafrœira da terra.

Arvore de Tucano. Pfefferfesserbaun, vide Turama.

Arvore de vacea. Kuhbaum, vide Massarandube.

Ascalote. Allium escalonicum L. Liliaceate.

Assa peixe. Fischasant. Vernonia ferruginea Lees. Compositae.

Bliithen.

Assa peixe branco. Weisser A. Ternonia daphnoides Wallp.

Compositale. Blätter und Bläthen.

Assa peixe do campo. Prärieasant. Veronial rufescenss sichlecht.

Compositile. Blïthen.

Assa peixe grande. Grosser Isant. Bohemerial candatil Sw. Ir-

ticaceae. Blüthen und Blätter.

Issacì. Giftbaum. Hura crepitans Müll. Arg. Euphorbiarceale.

Milch und Rinde bei Elephantiasis.

Assacu-mirim vide Jequirity.

Assafrôa. Guarea trichiloides L. Meliaceae. Rinde und Blätter. 
Assafroeira. Saffranbaum. Nycthanthes arbor-tristis L. Jasmineae. Bliithen zum Färben der Speisen. In Pernambuco ist es Stachytarpha glauca Sauer. Verbenaceae.

Assafroeira da serra. Gebirgssaffran. Escobodia scabrifolia R.

et P. Srrophularinae.

Assafroeira da terra. Einheimischer Saffran, vide Urucú.

Assahi. Speise von der Pulpe der Friichte von Assai, mit /ucker und Mandiocmehl.

Assai. Enterpe olerarea Mart. Palmae.

Assai-ai. Saure Assai. Euterpe olerarea Mart. Var. minor Dr.

Palmae. Palmkolıl.

Assai catinga, Euterpe ratinga Wall. Palmae. P'almkohl.

A ssai mirim. Kleine $\Lambda$. Euterpe precatorial Mart. Palmae. Palmkohl.

Assai-rana. Falscher A. Geonoma camana Trail. Palmae.

Assucará. Urwaldbaum. Bauhol\%.

Assucena. Amaryllis principes Salm. Dyk. Amar.llidareae. Zwiebel und Zierpflanze.

Assucena do brejo. Sumpflilie. Hedychium coronarium I. Zingiberaceae. Bliithen zu Parfiim, Wurzel aromatisch.

Assucena grande vide Trombeteira.

Assucena do in ato vide Larangeira do mato.

Astia. Vrwaldstrauch. Aeste zu I anzenschäften.

A taubá. Guarea tuberulata Vellos. Var. purgans DC: Meliareae. Rinde Abfiihrmittel.

A tapú vide Uatapí.

A tchá. Begonia rordata Vellos. Begoniareae. Blattsaft.

A tiriba. Bauholz, nicht bestimmt.

A titará vide Rutin.

A tolé vide ITrucú.

Atta. Beneunung fïr fructa de conde und fructa de condessa.

A turá und Aturia. Drepanocarpus lunulatus Mey. Papilionaceae.

Rinde officinell. Zweige zum Korbflechten zum Transport der

Mandioccawurzeln bei der Ernte derselben, welche Körbe von den

Indianern so benannt werden.

A urapá. Eine Heilpflanze im Staate Parà.

A urataceú. Eine Heilpflanze im Staate Amazonas.

A ï u va vide Aiïba.

A varamo und Avaremo-temo vide Abaremo-temo. 
Avaró vide Tucuma.

Araty. Tupiname für Mais.

Areio do mato. Wilder Hafer. Avena quadridentula Doll. Gramireae.

Areloz. Euphorbia heterodoxa Müll. Arg. Euphorbiaceae. Milch als Heilmittel bei Krebs.

A venca. Adiantum subcordatum Sw. Filices und Cheilanthes radiata R. Br. Filices.

Arenca grande. Grosse A. Adiantum trapeziforme L. Filices.

A venca miuda. Kleine A. Adiantum cuneatum Langsdf. Filices. A venca da Serra. Gebirgs A. Cheilanthes regularis Mett. Filices. Avenca sylrestre. Wilde A. Polypodium aureum L. Filices.

Avencão. Grosse A. Adiantum caudatum L. Filices.

A vencão da terra. Einheimische A. Cheilanthes rhlorophylla Sw. Filices.

A ven qu in ha. Kleinste A. Gymnogramme calomelanos Kaulf. Filices. A rinhado vide Merindiba bagre.

Avirama. Tupiname für Salgueira de Rio.

Axi. Indianische Benennung für Capsicum annuum L.

Axiotle vide Urucú.

Axúa. Unbestimmt. Bauholz im Staate Amazonas.

Ayapana. Eupatorium triplinerve Vahl. Compositae. Antidot bei Schlangenbiss.

A yapana venenosa. Euphorbia cotonoides Miq. Euphorbiaceæ. Milch und Rinde giftig.

A ymontabou. Montabea guyanensis Aubl. Ebenaceae. Bauholz. A you. Nectandra raga var. vulgaris Meissn. Lauraceae. Bauholz. A ypim ride Aipim.

Asmará-posoqueri. Posoqueria longifolia Aubl. Rubiaceae.

Frucht essbar. Bauholz.

A yri vide Brejauba.

A za de morcega. Fledermausflügel. Martia parvifolia Bth. Caesalpineae. Die purpurrothen Hülsen Heilmittel.

A za de morcega de folha de grande. Grossblätterige Fl. Malanea Martiana Müll. Arg. Rubiaceae. Wurzel Volksmittel.

Azedas. Sauerpflanze. Hibiscus digitatus Cav. Malvaceae. Zum Ansäuern der Speisen.

Azedinho. Sauerampfer. Rumex acetosella L. Polygonaceae. Speise. Wurzel Heilmittel. 
A zed in h!o ass ù. Grosser s. Begonia paleata A. DC. Begoniaceae. Heilmittel.

Azedinho do brejo. Sumpf S. Begonia acida Mart. Begoniaceae.

Heilmittel. '/um I’utzen von Metal.

A zedinho do campo. Präie Sauerklee. Oxalis hirsutissima Mart.

Oxalideate. Heilmittel.

Azedinho grama. Gras Sauerklee. Oxalis calva Prog. Oxalidear.

Die kleine Wurzelknolle wird roh gegessen.

A zedinho grande. Cirosser Sauerklee. Oxalis Aeripiens Prog.

Oxalidear. Heilmittel.

A zedinlo dos () urives. Goldschmidtsauerklee, vide Azedinho de brejo.

A zeite de cabumba. Balsam von Isiropsis insignis Engl. Burseraceate.

A zeitona da terra. Einheimische Olive. Hipluso on nitidus lot.

Lythracear. Frucht benutzt.

I zoito cavallo. Pferdepeitsche. Lnlwa grandiflora Mart. Tiliarear.

Rinde officinell. Blätter zum Schwarzäiben. '/weige zu Ruthen. A zoito cavallo de varetas. Pferderuthen. Luhea divaricati

Mart. Tiliacear.

A zougue. Quecksilber, vide Tayuya.

A zougue dos pobres. Quecksilber der Armen, vide Velame.

Azougue vegetal. Vegetabilisches Quecksilber, vide Manacan.

A zulao. Nicht bestimmt. Violetröthliches Möbelholz.

Bâba. Speichel, vide Coquinho bâba.

Baba de boi. Ochsenspeichel. Cocos Martiana Dr. et Gl. Palmae.

Frucht essbar. Blätter liefern Faser.

Baba de boi de campina. Malvaviscus rorlatus DC. Malvaceae.

Malwenansatz.

Baba de boi do grande. Grosse O. vide Maria Rosa.

Baba de moza. Jungfrauenspeichel. Eine delicate sïsse Dessertspeise aus dem Safte des Fleisches der Cocusmuss, Eier etc.

Babado. Datura suaveolens B. Br. Solanaceae. Giftpflanze.

Baboatan. Bauholz im Staate Pernambuco.

Babosa. Aloe Barbadensis Mill. Liliaceae.

Baboso vide Babado. Beide Wörter bedeuten Geifer, wie beim tollen Hunde.

Baboré vide Laranjinha do mato. Tupiname. 
Babunha. Guilielma speciosa Mart. Palmae. Frucht Nahrungsmittel.

Bacaba. Oenocarpus Bacaba Mart. Palmae. Fruchtpulpe zur Nahrung. Samen zu Oel.

Bacaba-aky ride Bacaba mirim.

Bacaba-assú. Grosse B. vide Bacaba.

Bacaba-i. Oenocarpus multicaulis Spruc. Palmae. Samen Oel liefernd.

Bacaba mirim. Kleine B. Oenocarpus minor Mart. Palmae. Fruchtpulpe zu erfrischendem Getränk.

Bacabada. Speise aus dem Fruchtfleische der Bacabafrucht.

Bacaiúba. Acrocomia glaucophylla Dr. Palmae. Frucht essbar.

Samen Oel liefernd.

Bacam arte unbestimmte Heilpflanze.

Bacamicha. Bumelia obtusifolia R. et R. var. excelsa Eichl.

Sapotaceae. Rinde bitter als antifebril.

Bacayuva. Acrocomia glaucophylla Dr. Palmae.

Bacayma do bryo. Acrocomia odorata Barb. Rodrig. Frucht essbar.

Bacomisca. Sirleroxylon vastum Fr. Allem. Sapotaceae. Bauholz. Bacana. Pflanze gegen Rheumatismus.

Bacopari. Platonia insignis Mart. Clusiaceae. Frucht essbar.

Bauholz.

Bacopari amarello. Gelbe $B$. vide Buranhem.

Bacopari arvore. Baum B. vide Larangeira do mato.

B a copari assì. Grosse B. Salacia crassifolia Peyr. Hippocrataceae.

Frucht essbar. Blätter officinell enthalten Salacin oryst.

Bacopari assí vide auch Aymará-posoqueri.

Bacopari bravo. Wilde B. Salacia arborea Peyr. Hippocratareae. Fruchtpulpe essbar. Blätter officinell.

Bacopari do campo. Steppen B. Salacia dulcis Bth. Hippocrataceae. Frucht essbar.

Bacopari da capoeira. Gebüsch B. Salacia campestris Walp. Hippocrataceae. Frucht essbar.

B a copari cipo. Lianen B. Salacia sylvestris Walp. Hippocrataceae. Frucht essbar. Blätter Heilmittel.

Bacopari do mate. Wilde B. Salacia micrantha Peyr. Hippocrataceae. Frucht und Blätter Heilmittel. 
Bacopari miudo. Kleine B. Coccocypselum canescens Willd. Rubiaceae. Frucht essbar. Blätter Heilmittel und Rheedia

Gardneriana Planch. et Trian. Guttiferae. Frucht essbar. Bacopari da Serra. Gebirgs B. vide Salacia crassifolia Peyr.

Bacor $\widetilde{\iota} o$ vide $A$ potia-coraca.

Bacori vide Bacopari.

Bacuri. Rheedia macrophylla Planch. et Fr. Guttiferae. Frucht essbar.

Bacuri-membeca. Die Früchte des Baumes dienen den Indianern zur Speise.

Bacurubú und Bacuruby vide Bandarrá.

Bacurúpari vide Bacopari.

Bago de alfarroba. Johannisbrodbeere vide Canella do veado branco.

Bago de anil. Indigobeere. Solanaceae. Zum Färben baum wollener Zeuge.

Bago azal. Blaue Beere. Psychotria pleiocephala Müll. Arg. Rubiaceae. Bauholz. Beeren zum Färben.

Bago de chumbo grosso vide Bauna. Grober Schrot.

Bago de chumbo miudo. Feiner Schrot. Cardiospermum parviflorum St. Hil. Sapindaceae. Heilmittel bei Keuchbusten. Die Benennung beider zufolge der Bleikorn ähnlicher Samen.

Bago de louro amarello. Gelbe Lorbeerbeere. Nectandra nitidula Nees Lauraceae. Bauholz und aromatische Frucht.

Bago de periquito. Sittigbeere. Unbestimmtes Bauholz in Santa Catharina.

Bago da praia. Küstenbeere. Coccoloba uvifera Jacq. Polygonaceae. Frucht essbar. Samen adstringens.

B aguassì. Orbignia Martiana Barb. Rodr. Palmae. Corrumpirtes Guaraniwort: Uá-Frucht, uassú-gross.

Bafureira. Wilder Feigenbaum vide Carrapateira.

Bainha de espada. Säbelscheide. Acanthinophyllum strepitans Fr. Allem. Urticaceae. Bauholz.

Bakopary. Rheedia floribunda Pl. et Fr. Guttiferae. Frucht essbar.

Balatá. Couratari Estrellensis Radd. Myrtaceae. Bauholz. Samen oelreich.

Balatá assu. Grosse B. Mimusops balata Gaertn. Sapotaceae. Milchsaft. Bauholz. Spec. Gew. $=1,062$. 
Balatá branca. Weise B. Conratari guianensis Aubl. Myrtaceae. Bauholz. Schiffsholz.

Balieira vide Capiximgui.

Balieira branca. Weisse B. Cordia rylindrostachys Fr. Allem-

Cordiaceae. Blätter officinell.

Balsamo. Myrospermum erythroxylum Fr. Allem. C'aesalpinceae. Vorzïgliches Bauholz. Rinde und Balsam officinell vide auch Oleo de mico.

Balsamo de Carana. Caranabalsam. Protium Carana March.

Burseraceae. Frucht essbar, Harz officinell, Bauholz.

$\mathrm{Balsamo}$ vermelho, vide Oleo vermelho.

Balsamona. Cuphea balsamona Cham. A. Schl. Lythrareap. Antifebril.

Bamb a vo vide Melancia da praia. Gleiche Benennung hat auch der

Fruchtzapfen der Jacafrucht vide Jaca.

Ba mboré vide Laranginha do mato.

Bamborral vide Herva de Santa Maria.

Balsedo. Benennung im Staate Marauhao für Aquapé.

B a mbú. Bambusà vulgaris Schrad. Grumineae.

Bambú de Bahia. Bambusa gigantea Walp. Gramineap.

Bambú da China. Bambusà arundinaceae Walp. Gramineae.

Bambú do mato. Nastus barbatus Rupr. Gramineae.

B ambusinho vide C'arizó.

Banana. Musa paradisiaca L. Musaceap. Frucht etc.

Banana brava. Wilde B. Heliconia Bihai Sw. Musaceas. Frucht. Banana do bryo. Sumpf B. Caladium striatipes Schott. Araceae.

Frucht Abführmittel.

Banana do campo. Steppen B. vide Colher de vaqueiro.

Banana imbé vide Banana de macaco.

Banana do mato. Wald B. vide Pepina de papagaio.

Banana de macaco. Affenbanane. | Philodendron bippinBanana de morcega. Fledermausbanane. $\zeta$ natifidum Schott. Araceap. Frucht essbar. Lieblingsfrass der Affen und Fledermäuse. Banana de papagaio. Papageien B. Apidosperma australis Mull. Arg. Apocynaceae. Bauholz. Rinde Heilmittel.

Banana de sementes. Samentragende B. Musa rosea Jacq.

Musaceae. Samen bohnengross.

Banana de S. Thomé vide Banana.

Banana sylvestre. Wilde B. Canna brasiliensis Spr. Cannaceae. Samen als Halsschmuck. 
Banana da terra. Einheimische? B. Musa sapientum L. Musaceae. Bananeirinha. Kleine B. Canna aurantiaca Rosc. Cannaceae. Wurzel und Same.

Bananeirinha do mato vide Albarà.

Bandarrà. Schizolobium excelsum Vog. Caealpinieae. Holz zu Flössen.

Barabú. Bauholz im Staate Pernambuco.

Baracatuto vide Orelha da onça.

Barahuna. Melanoxylon Braúna Schott. Caesalpinieae. Unzerstörbares Bauholz.

Barajúba. Bauholz im Staate Parà.

Barba de barata. Schabenbart. Caesalpinia pulcherrima Sw. Caesalpinieae. Vorzügliches Bauholz.

Barba de bode. Bocksbart. Aristida pallens Cavan und Ctenium (irrhosim Kth. Gramineae, ferner Picrosia longifolia D. DC. Compositae.

Barba de bode do campo. Steppenbocksbart. Thylothrasya petrosa Doll. und Ctenium cirrhosum Kth. Gramineae.

Barba de boi. Ochsenbart. Eriantlus asper. Nees. Gramineae und Ramirea maritima Aubl. Cyperaceae.

Barba de cabra. Ziegenbart. Parinarium obtusifolium Hook. fil. Rosaceae. Rinde febrifugum.

B arba de camaroa. Krabbenbart. Strychnos rubiginosa A. DC. Loganiaceae. Samen und Wurzel officinell.

Barba de Lagôa. Seebart. Enhydra integrifolia Gardn. Conpositae. Heilmittel.

Barba de S. Pedro. Petersbart. Polygala paniculata L. Polygalaceae. Hustenmittel.

Barba de velho. Greisenbart. Tillandsia usneoides L. Bromeliaceae. Matratzen-Füllung.

Barbante vegetal. Vegetabilischer Bindfaden. Rhipsalis teres Steud. Cactaceae.

Barbasco. Wollkraut. Buddleia brasiliensis Jace. - B. brachiata Cham et Schl. B. elegans Cham et schlecht. Scrophularinae. Emolliens.

Barbasco do mato. Wildes Wollkraut. Pterocaulon spicatum DC. Compositae. Heilmittel.

Barbasco de peixe. Fisch Wollkraut. Jacquinia armillaris Jacq. Myrsinaceae. Zum Betäuben der Fische. 
Barbatimao. Stryphnodendron Barbatima Mart. Mimoseae.

Rinde zum Gerben. Bauholz.

Barbatimao branco. Weisse B. Stryphnodendron polyphyllum

Mart. Mimoseae. Pulpe der Schote Abfïhrmittel; Rinde zum Gerben; Bauholz.

Barberinha vide Aperta.

Barboleta. Schmetterling. Zierpflanze in Pernambuco.

B arbusano. Apollonias canariensis Nees. Lauraceae.

Baririço vide Maririço.

Baririço do campo xide Batatinha do campo.

Barriguda. Dickbauch, zufolge des angesihwollenen Stammes.

Chorisia crispiffora Kth. Bombaceae. Samenwolle. Samen ölreich. Barriguda do sertao vide Embiratanha.

B arnù. Dipțeris alata Vog. Papilionaceae. Aromatische Samen und Rinde.

Basilicao. Ocimum basilicum L. Labiatae. Aromatisch. Anthelmintivum.

Bastro de brejo. Richardsonia brasiliensis Gomes. Rubiaceap.

Wurzel als falsche Ipecacuanha.

Batata do ar. Luftknolle. ; Cissampelos fasciculata Benth.

Batata brava. Wilde Kartoffel. $\}$ Menispermareae. Wurzel officicinell. Antidot bei Schlangenbiss.

Batata de caboclo. Indianer Kartoffel. Bignonia exoleta Vellos.

Bignoniaceae. Knollen werden von den Indianern genossen.

Batata do campo. Steppenknolle. Gesnera alagophylla Mart.

Gesneraceae. Knolle Heilmittel.

Batata doce. Süsse Kartoffel. Ipomoea batatas Ian. Convolvulaceae.

Batata doce amarella. Gelbe süsse K. Ipomoea hatatas Lam.

var. xanthorhiza. Chois. Convolvulaceae.

Batata doce de Demerara und B. d. de Angola. Ipomoea batatas Lam. var. porphyrorhiza Grieseb. Convolvulaceae

Batata de enfieira. Aufgereihte Kartoffel. Ipomoea argentea

Meissn. Convolvulaceae. Knöllen als Heilmittel.

Batata do mar vide Salsà da praia.

Batata ovos. Eierknolle vide Trapoerava-assú.

Batata de purga. Purgier Knolle. Operculina altissima Meissn.

Convolulaceae. Ersatz der Radix Jalappae.

Batata da terra. Einheimische Kartoriel vide Batata doce. 
Batatá von yba-Frucht, tatífeueroth. Lncmma Beanrepairei R. et Glas. Sapotaceae. Bauholz. Frucht essbar.

Batatinha. Kleine Knolle. Ipomoea I'eckoltii Meissn. Convolvinlareae. Knolle als Schlangenantidot.

Batatinha ca a peba. Knöllchen des grossen Blattes vide Batata brava.

Batatinha do eampo. Steppenknöllehen. Polılia bonariensis Per. Iridaceae. Abführmittel.

Batatinha contra. P'olygalil violoirdes st. Hil. I'olygalareate. Schlangenantidot.

Batatinha do mato. Waldknöllehen. Ipomoral geranioirles Meissn. Convolvulacease. Abfïhrmittel.

Batatinha de purga. Abfïhrköllchen. Ipommia rissoirles Grieseb. Convolvulaceas.

Baté testa vide Camapú.

Batinga. Eugenia clinocarpar. DC. Myrtaceae. Bauholz.

Batinga branca. Weisse 13. Engenia apiocalpa Bg. Myrtaceae. Bauholz.

Batinga rasteira. Kriechende B. Eugenia polycarpa var. bimarginata $\mathrm{Bg}$. Mrrtaceae. Adstringens.

Batinga vermelha. Rothe B. Eugenia durissima Fr. Allem. Myrtaceae. Bauholz.

Bati-puta. Ouratea coccinea Engl. Ochnaceae. Oel der Samen officinell.

Bati-puta mirim. Kleine B. )

Bati-puta bravo. Wilde B. $\}$ vide Jabotapita mirim.

Battaua. Oenocarpus Batawa Mart. Palnae. Samen liefern Speiseöl.

Battan. Andradaea floribunda Fr. Allen. Nvctagineae. Bauholz.

Battaw a vide Battaua.

Batuéra. Enthïlster Maiskolben in Tupisprache.

Ba u b vide Ambaiba branca.

Bauna. Cardiospermnm corindum L. Sapindaceae. Keuchhustenmittel.

Baunilha. Vanilla planifolia Andr. und Vanilla aromatica Sw. Orchidaceae. Vanille liefernd. Officinell.

Baunilha da Bahia. Tanilla palmarum Lindl. Orchidaceae. Vanillon liefernd.

Ba unilha brava. Wilde V. Vanilla Chamissonis Klts. Orchidaceae. 
Ba unilhao. Grosse Vanille. Vanilla pompona Schied. Orchidaceae. Vanillon liefernd.

Bavana. Unbestimmte Pflanze. Zu Bädern bei Rheumatismus.

Bayacú. Heilpflanze im Staate Bahia.

Baxiúba. Iriarrtea exorhiza Mart. Palmae.

Baxiúba barriguda. Bauchige B. Iriartea ventricosa Mart. Palmae.

B a ýu curú. Liliaceae. In Staate Rio Grande do Sul bei Wassersucht. Becuiba. Myristica heruyba Schott. Myristicaceae. Saft als Kino, Rinde, Macis und ölreiche Samen officinell. Bauholz. Spec. Gew. $=0.776$.

Becuiba branca. Weisse B. Myristica Gardneri A. DC. Myristicaceae. Frucht und Bauholz.

Becuiba cheirosa. Wohlriechende B. Myristica theiodora Spruc. Blätter, Frucht und Holz.

Becuiba do Pará. Myristica surinamensis Rol. Blätter, Früchte und Holz.

Becuiba redonda. Runde B. Myristica officinalis Mart. Blätter, Früchte, Rinde und Holz.

Becuib-assú. Grosse B. Terminalia lncida Hoffmsegg. Combretaceae. Friichte und Bauholz.

Beeberú vide Bibirú.

Begonia de batata. Knollen B. Gloxinia atteuuata Hast. Gesneraceae. Knollen.

Begonia grande. Grosse B. Begonia platanifolia Grah. Begoniaceae. Blätter officinell.

Beijo do mato. Wilde Kussblume. Phaseolus bracteolatus Nab. E. Papilionaceae. Zierpflanze.

Beijo de moça. Mädchenkuss. Bid ns bipinnatus L. Compositae. Zierpflanze.

Bejoeiro. Benzoebaum. Pamphilia anrea Mart. Styraceae. Harz und Rinde.

Beijú. Tupibenennung für Kuchen von Mandioccamehl.

Beijú-cica. Kleine Mandioccakuchen.

Beiju-curuba. Mandioccakuchen mit dem Nusskern von Bertholletia excelsa.

Beijú-guassì. Stark geröstete Mandioccakuchen.

Beijú-membecca. Wenn die Masse der Mandioccakuchen durch Gährung ein poröses Gebäck liefert. 
Beijú-puquequa. Mandioceateig gesalzen und auf Bananenblättern elwärmt.

Beijú-teyca. Mandioccateig mit Stärkemehl gemischt und gebacken.

Bejuca de veado. Paragonia pyramirlarlis Bur. Bignoniaceae.

Beldroega. Portulacca oleracea. L. Portulaccaceae. Gemüse.

Bellas vide Cipo de S. Joao.

Bellas noites vide Maravilha.

Bellas sombras. Schöner Schatten. Pircunia dioica Miq. Phytolaccaceae.

Bem casado. Gut verheirathet, zufolge der doppelten Blumenkrone.

Datura fastuosa I. Solanaceae. Giftpflanze.

Bem casado sylvestre. Wilde, vide Pinheirinho dagua.

Bem me quer. Maassliebchen. Aspilial foliacal Bak. Compositale. Blätter.

Bençao de Deos. Gottessegen. Abutilon purpurascens K. Sch. Malvaceae; im Staate S. Paulo ist Abutilon Berlfordianum St. Hil. var. discolor K. Sch.

Beringela. Solanum melongonil L. Var. ovigermm Dun. Solanaceae. Frucht als Gemiise.

Beque cheiroso. Artanthe tulserculata Miq. Piperaceae. Blätter und Wurzeln Heilmittel.

Betonica. Eriope crassipes Benth. Labiatae. Arom. Heilmittel.

Betys. Artanthe colubrina Miq. Piperaceae. Blätter officinell.

Bexuquilho vide Ipecacuanha.

Bibirú. Nectandra Rhodiei Schomb. Lanrareate. Rinde antifebril,

Alkaloid Beebirin. Schiffs- und Bauholz. Spec. Gew. 1.600.

Bico de corvo. Rabenschnabel, vide Alcassus bravo.

Bico de pato. Entenschnabel. Kylosma Salzmanni Eichl. Bixaceae; ferner noch mit demselben Volksnamen: Machaerium lanatum Tul. und M. discolor Vog. Papilionaceae. Bau- und Möbelholz.

Bicorapia. Essbare Frucht eines Strauches im Amazonengebiet.

Bilimbi. Averhoa bilimbi L. Oxalidaceae. Frucht essbar.

Bilros. Amaryllis nivea Röm. et Schult. Amarillidaceae. Zwiebel Heilmittel.

Biribá. Daguetia Marcgraviana Mart. Inonaceae. Frucht essbar. Faser und Bauholz.

Biribarama. Rollinia orthopetala A. DC. Anonaceae. Bastpflanze. Biribi. Anona sericea Dun. Anonaceae. Frucht essbar.

Biriti. Mauritia flexuosa Linn. fil. Palmae. 
Biroró. Süsses Gebäck von Mandioccamehl, Zucker und Anis.

Bir ú vide Imbiri.

Bisnaga. Wilde Mohrrübe. Ammi bisnaga Lam. Umbelliferae. Sympathiemittel.

Bledo. Amaranthus spinosus L. Amaranthaceae. Gemüse.

Bôa noite. Gute Nacht, vide Acajavana.

Bôa tarde. Guten Abend. Oenothera acaulis Cavan. Oenotheraceae. Zierpflanze.

Bóbó. Speise in Bahia von gelben Bohnen, Bananen und Elaeis guinensis Oel.

Bocajà. Acrocomia totai Mart. Palmae. Frucht und Stamm. Bocay ûba vide Macahuba.

Bocea de sapo. Froschmaul. Deganira erubescens Cham. et Schl.

Gentianaceae. Ersatz für Enzianwurzel.

Bogaró. Iasminum sambac Vahl. Iasminaceae. Blätter u. Blüthen. Boia-caa und Boia-cica vide Paracary.

Boi gordo vide Alcassus bravo.

Bolsa de pastor. Hirtentasche. Zeyhera montana Mart. Bignoniaceae. Officinell die bitteren Samen und Wurzelrübe.

Bom nome verdadeiro. Elaeodendron quadrangulatum Meissn.

Celastrinaceae. Rinde und Holz.

Bom nome bravo vide Jabotabita.

Bonéca. Benennung des blühenden Maiskolbens.

Boniato. Nectandra exaltata Griesb. Lauraceae. Bauholz.

Bonina vide Maravilha.

Borbo vide Rabo de tatú.

B ord ao da velha. Greisinenstab. Pithecolobium glomeratum Benth.

Mimosaceae. Schoten officinell. Bauholz und Stöcke.

Bordao da velha de feijao. Pithecolobium campestre Spruc.

Mimosaceae. Samen, Bauholz.

Bordao sinho. Kleiner Stab. Secondatia densiflora A. DC. Apocynaceae.

Bor oré. Pfeilgift am Amazonenstrome.

Borracha. Hevea brasiliensis Müll. Arg. und H. guinanensis Aubl. Euphorbiaceae. Cautchouclieferanten.

Borragem chimarona. Echium plantagineum I. Borraginaceae. Officinell.

Boruleo. Borulea campestris Fr. Allem. Urticaceae. Milch und Frucht. 


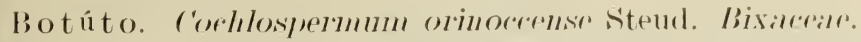

Bouquet do mato. Waldblumenstranss. I'isonia sulneorlata siw.

Nuctaginareare.

Bouquet da noiva. Brautblumenstrauss. Śpirada rhamiledrifolial

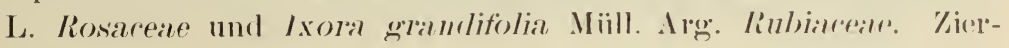
phanze.

liraço de preguica. Faulthierarm. Solimmm rermmm Vellos.

Solanareac. Heilmittel.

Brandão. Pflanze im Staate l'aura als antisyphilinum.

Branquilho. Bauholz.

Brasileto vide Paì Brasil.

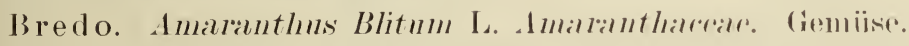

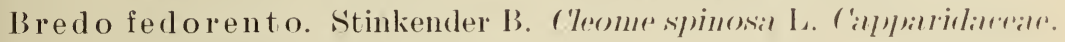

Blumen und samen.

Brejauba. Astrocarymm Ayri Mart. I'almare. Frucht essball. Holz zu Bogen der Indianer.

Breo branco. Baum in Staate I'ara. Harz und Rinde.

Brilhante. Lagenocarpus atamantinns N. ab. E. Cryeratedar.

Brincos. Vochysial gummiferal Mart. Vorhysiaceare (iummi und

Bauholz.

Brinco de coral. Stephanophytum longifolimm Pohl. Acanthactale. Brinco de Sahoim vide Abaremo-temo.

Broca. Striegel. Rhedia hrasiliensis Planch. et Fr. Gintiferar.

Frucht essbar, Milch officinell. Bauholz.

Broma. Brunfelsia obovata Benth. Seropularinar. Wurzel off.

Broma branca. Weisse B. Alectra lorasiliensis I enth. Seroplunlarinae. Wurzel emetico-cathartic.

Broma rosca. Violetrothe B. Beyrichia florilundal Benth. Siropliularinze. Wurzel.

Bubunha. Guilielma speciosal Mart. Palmax. Frueht Nahrung der Indianer.

Bucarè. Erythrina glaucal IVilld. I'apilionaceap. Rincle enthält Erythrinin. Schattenbaum.

Bucha. Luffia aegyptiaca Roxh. C'ncurbitareae. Fasergewebe der Frucht.

Bucha campista. Luffa acutangula Roxb. C'ncurbitacex. Unreife Frucht zu Gemüse, reif das Fasergewebe.

Bucha do mato. Gurania. Panlista Cogn. C'ucurbitacex. Frucht als drasticum. 


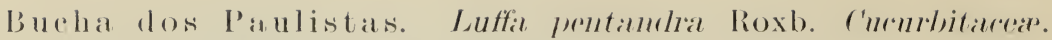

Frucht als drasticum, das Gewebe zu Flintenpfropfen.

bucha de rad vicle Camapú.

liucha de viado. Amajonal sineriforal Mart. Rubiacex. Rinde.

Buchinha. Inflir operculata Cogn. Onrmbitacear. Frucht starkes irasticum.

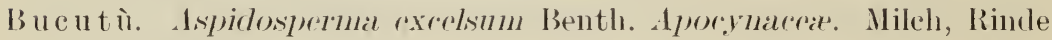
off., Bauhol\%.

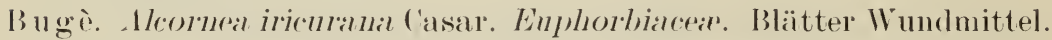
Bauholz.

Bugi und Bugio. Combretum Jacronimii Grieseb. Combretacear. Rinde. Bauliolz.

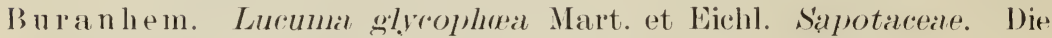

Rincle ist die off. ('ort. monesia. Friichte off. Bauholz.

Buri. Diplotheminn randescens Mart. Pahmae.

Buri-cica. Baum in Staate Pernambuco, das Holz dient zu Zuckerkisten.

Buriti. Manritia vinifera Mart. Palmae. Früchte essbar; der Saft des stammes zu P'almwein.

Buriti bravo. Wilde B. Manritial armata Mart. Frucht essbar; Stacheln ersetzen den Indianern die Nadeln.

Buritizaba. Süssigkeit bereitet aus den Buritifrüchten.

B и r r le iteira. Milchgebende Eselin. Exraecaria arguta Mïll. Arg.

Enphorbiaceare. Milch und Rinde toxisch.

Burueh. Brosimum discolor Schott. Crticarear. Früchte u. Mileh werden vom Volke genossen.

Bussì. Manicartia silcciferal Gärtn. Palmale. Faserstoff.

Butereiro. Butterlaim. Büttneria ratalpifolia Jacq. Sterculiaceae. Samen sehr ölreich.

Butia. Cocos capitata Mart. C. odoratal Barb. Rodr. Palmate. Frucht essbar.

I utia assì. Grosse 13. Cocos pulposia Barb. Rodr. Palmate. Frucht essbar.

Iintia do eampo. / Cocos eriospatha Mart. Palmae. I3 utia do Rio Grande do siul. I Frueht essbar. Aus der Pulpe wircl Geträink bereitet.

Butua. Aluta rufescens Aubl. Menispermareae. Wurzel off. liutua do corvo. Raben B. vide Algodoeira do campo. Butua legitima vide Abutua. 
Butua miuda vide Abutua miuda.

B u tu in ha. Cissampelos glaberrima st. Hil. Menispermareae. Wurzel Antidot bei Schlangenbiss.

Buxina. Schiffs- und Bauhol\% in den Nordstaaten.

Buxo de boi. Zephlera tubercnlosa Bur. Bignoniareate. Fruclit ofit.

Cá a. Tupibenennung für Blatt, Kraut, ferner and der imlianische Name für Mate und für Fumo bravo.

Caa-acapoc vide Acapurana

Caa-apia vide Contra herva.

Caa-apicum ) Rhabrlia licioirles Mart. Heliotropiesn. Pflanze als Caa-picì. $\{$ Heilmittel.

Caa-assu. Grosses Blatt. Bytsonima crissifolia Kth. Malpighliarear.

Frucht und Blätter.

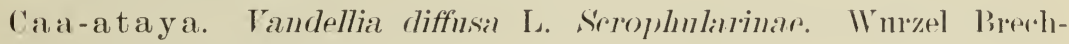
mittel, emmenogogum.

Ca a-atia vide Herva de Santa Lmyia.

Caa-cann a vide Yquetaia.

Caa-chira vide Anil miudo.

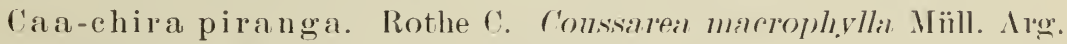

Rosaceas. Wurzel rothen Farbstofit.

Caa-cica. Enphorbia pilnliferal L. Enphorlbiareale. Pflanze ofit.

Ca a-cuis. Tupiwort fïr die Blattkmospen des Matebaumes.

Ca a-eo vide Malicia de mulher.

Caa-ghuara. Indianischer Ausdruek, wenn die Mateknospen sich vollständig geöfinet.

Ca a-gua-cuiba. Unbestimmtes IBïmohen, dessen solwarme fleischige Friichte ein Lieblingsfressem der Vägrol.

$\left.\begin{array}{l}\text { Caa-guiynyo } \\ \text { Caa-guiynyo-tó }\end{array}\right\}$ virle Aninga pari.

Caa-jandiwap vide Loco.

Caa-jassá vide Tameorana.

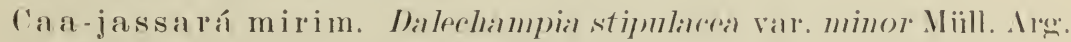

Enphorbiaresale. Bliatter officinell.

Ca a -kera vide Anil miudo.

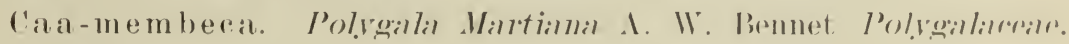

Wurzel.

Can-mendâ vide Flor de rasamento.

('a a-mirim. Kleines Mateblatt. 
Ca a-ınniana. Lophogyne helicandra Tul. Podostemaceae. Pflanze officinell.

Caa-ngai. Belbunium noxinm Sch. Rubiaceae. Wurzel zum Rothfärben.

Caa-opia. Farbiges Blatt. Vismia guianensis Chois. Guttiferae. Milch und Rinde drasticum.

( a a-opia do campo. Farbiges Blatt der Steppe. Vismia micrantha Mart., V. rufescens Pers. und I. baccifera Reich. Gnttiferae. Milch und Rinde drasticum.

Caa-peba. Grosses Blatt, vide Pariparoba. Dieselbe Benennung hat Cissampelos glaberrima St. Hil. Menispermaceae. Wurzel off. Ca a peba do Norte. Potomorphe peltata Miq. Piperaceae. Wurzel und Blätter.

Ca a peba do Sul. Potomorphe sidaefolinm Miq. Piperaceae. Wurzel und Blätter.

Ca a-peúra vide Caa-peba.

Caapi. Banisteria caapi Sprue. Malpighiareane. Wurzel off.

Ca a pia assí. Grosse C. vide Carapia.

Ca apia mirim vide Contra herva.

Caapim. Tupiname für Gras.

Ca a pim-peba. Eleusine indica Gärtn. Gramineae. Futtergras.

Caa-pomonga vide Loco.

Caa-ponga. Iresine vermicularis Mag. Amaranthaceae. Blätter als Wundmittel.

('a a-pororoca. Myrisine ovalifolia Miq.-M. parvifolia $\Lambda$. DC.M. nmbellata Mart. var. arutifolia Miq. Myrsinacease. Frïchte und Holz.

('ali-poropoca assì. Grosise C. vide Casca d'Anta.

('aa-pororoca do campo. Steppen C. vide Perobinha.

('a a-pororoea miuda. Kleine C. Mylsine flormlosa Mart. u. M.

Giardneriana A. DC. M.risinaceae. Frïchte.

Caa-potiragô. Spermarore glalbra Mchx. Rubiareae. Wrurzel ofi. Caa-puza virle Camapù.

Caa-rerù. 'Tupiname für essbares Blatt vide Baldroega.

Caa-robôa. Tupiname für Jatubà.

('a a-ryma. Trupiname für Mandioccastïrkemehl.

laa-taya. Tupiname für Herva de bixo.

Ca a-tendy. Syilantlies niens lacq. Compositae. Plaitter brennend.

Caa-teninga. Tupiname fiir Mata pasto. 
Caatigua. Trichelia Catigua A. Juss. Meliaceate. Rinde off.

Ca a-v ù vide $\Lambda$ nil.

Ca avurana. Solannm Cavmana Vellos. Solanacrae. Blïtter off.

Caa-xia. Solannm pterocanlon Dund. Solanaceale. Blätter.

Ca $a-x i x$ a vide Aguarà-quiga.

('a a-yby-assù. Dipteralcanthlus schancrianus N. alb. E. Acanthatreare. Wurzel.

Caa-ycy. Tupiname fiir Icicahar\%.

Cabaceira. Stiftia parviffora. Don. Compositar. Rinde.

Cabaceira amargosa. Lagenaria rulgaris Ser. Curnrbitacente. Fruchtschale zu Gefaissen.

Cabaceira cheirosa vide Abobora malão.

Cab a cinho. Dermoph,vlla pendulina Manso. ('ucurbitaceae. Frucht drasticum.

Cabeça de cutia. Cutiakopi. Myriaspora Egensis I)(. Melastomacease.

Cabeça de frade. Klosterbuderkopf. Echinosartus ()ttonis Lehm. und E. temispinis Leh. et ()tto. Circtareate.

('abera de monge. Mönchskopf. Lafoensial armminatal I)(. L,vthraceare. Frucht.

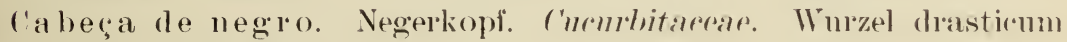
und antisyphiliticum.

('abelleira. Periickenbaum. ('arrar glahrnm Pers. Rhizoholeate. Samen fett. Bauhol\%. Spec. Gew. 1.187.

cabello de negro. Negerhaar. Erythroxylum mampestre St. Hil. Erythroxylameare. Rinde und Bauholz.

Cabello de negra. Negerinhar vide Arîriba do campo.

Cabelluda. Haarig. I'hivlloralix tomentosus Bg. Mrrtarean. Findit essbar.

Cabezuda. Dickkopf. C'oros malpitatal Mart. Palmae vide auch Acuri.

Cabiúna. Polisander. Lallorgia miseolobinm Benth. Papilionareaas. Möbelholz.

Cabiúma rajada. Gemaserter Polisanter. Dallbergia nigra Fr. Allem. Papilionaceàe. Möbelholz.

('abo de facao. Messerheft. Myriaria hrasiliensis Fr. Mllem. Mrrtaceale. Holz.

Caboatan. C'upanial vernalis St. Hil. Sapinclaceae. Bauholz.

l'a boatan de leite. Lithraea brasiliensis L. March. Anacardiaceae. Bauholz. 
$\left.\begin{array}{l}\text { Cabriuvinha do campo } \\ \text { Cabubreiù }\end{array}\right\}$ vide Cabureiba.

('abui. Enterolobinm Intescens Fr. Allem. Mimosareat. Baulıolz.

Spec. Gew. 0.672.

C'abui-iba vide Paì amarello.

Cabui-vinhatico vide Vinhatico de espinho.

Cabureiba. Balsambaum. Myrocaryns fistigiatus Fr. Allem.

Ciesalpiniaceap. Liefert Tolubalsam ähnliches Harz. Rinde off.

Vorzügliches Bauholz.

Cabuirei-cica. Balsam ron Cabureiba.

('a buy a vide Pita.

Caca-Henriqueta. Henriettea sureosa DC. Melistomaceae.

Cacao. Theobroma Cacao L. Büttmeraceae.

('aca o bravo. Wilde Cacao. Theolnoma speriosmm Willd. Büttnerareae.

Cacao do mato. Waldeacao. Theobroma subincanum Mart. Biittneraceae.

('acao miudo. Kleiner ('. Theobroma microrarpum Mart. Bïttneraceae.

Cacao do sertão. Wilder C. Theobroma hicolor Hb. et B. BüttCacaosylvestre. ${ }_{\text {C }}$ neraceate.

Cacaorana. Falscher Cacao. Theobroma sylrestre Mart. Büttneraceae.

Cacaú vide Orelha de onça.

Cacaú sylvestre vide Chicha.

Cachaoú. C'erens melanurus R. Sch. Cartareap. Otficinell, homeopathisch.

Cacharama. Bactris rlloracantha Poepp. Palmae. Frïchte essbar. C'achaxa. Zuckerrohrbranntwein.

Cachibú. Harz von Paú de porco.

Cachim. Excaecarta ilicifolia Spros. Euphorbiareate. Milch ätzent. Bauholz.

C'aço. Feingepulverter Schnupftabak.

('aculage vide Quitoco.

raet̀̀ vide Caite.

('afé bravo. Wilder Kafiee. Ginneral manirata Lindl. Haloragarea.

Frucht Volksinittel.

la fé de caboclo. Indianer Kaffee. Conssarea poroph.rlla Miill. Arg.

Rinbiareae. Frucht von den Indianern benutzt. 
Cafo eipo. Lianenkaffee. Ipomofia asaldifolia Röm. et sehleeht.

fonvolvuliueare. Geröstet zur Verfiilschung des gerösteten Kaffees.

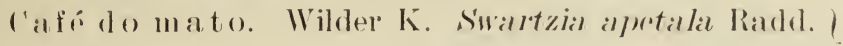

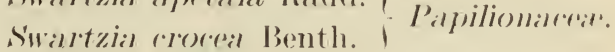

(ieroistete Simmen ein Volksmittel.

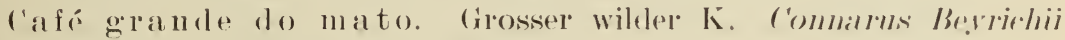

Blanch. C'omilnalraze. Bauhol\%.

Cafó mindo do mato. Kleiner willer K. Ixoral rertioillata Miill.

Arg. Rubiareale. Blätter Volksarznei. 'Zierpflanze.

('aferana. Falscher Kaffee. Tachia guianensis Aubl. Contianarate.

Wurzel specificum bei intermittirendem Fieber, sehr bitter, ent-

hiilt krystallinisches Caferanin.

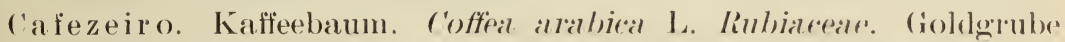

Brasiliens.

Cagaiteira. Diarrhoebaum. Stenocallix rlasentericus IBg. Myrtarar.

Der Genuss der Frïchte verursacht Drsenterie.

Cahatinga vide Piassaba.

('aha-xio. Cryptocaria guinnensis Meissn. Linnacear. Rinde aro-

maticum. Bauholz.

Cahiva. Unfruchtbarer Waldboden.

Cahuaia. Oreophoma subine. mis Dr. et O. tenue Mart. Palmate.

Caiaué. Elaeisis melinococea Gärtn. Palnae. Friichte ölreich.

laimbé. Cassapoa scabra Fr. Allem. P'apilionaceae. Baulolz.

Caimbé bravo. C'nratella americannal L. Dillemalceae. Blätter Volksinittel.

Ca in a na. Schlangenwurzel. Chiococear hrachiata R. et P. Var. acuminata Mïll. Arg. Rubiacere. Cainca. Schlangenantidot.

Caincar. Weisse Beere. Chiococcal brachiata R. et. P. rar. genuina

Miill. Arg. Rubiacrae. Officinelle Rad. Caincae.

Cainca do campo. Steppencainca. Chiococea brachiata li. et I'. var. diplomorpha Müll. Arg. Rubiaceae. Wurzel Antidot bei schlangenbiss.

Caimito und Cainito. C'hrysophyllum cainito L. Siapotaceae. Frucht essbar. Bauholz. Spec. Gew. 0.938.

Caimito da serra. Gebirgs ('. Montubea aculeata R. et P. Eloenaceae. Bauholz.

Caiotia vide Caa-cica.

Caipuna. Mvrcia tingens Bg. Myrtaceate. Blätter arlstringens, dienen zum Färben baumwollener Zeuge. 
l'a ipunga vide Coerana.

L'airussú. Malonetia sessilis Müll. Areg. Aporrnaceae. Milch und Holz benutzt.

C'a irussú do brejo. Śmmpf ('. C'entella asiatica Lrban. L'mbelliferre. Tolksarznei.

C'a issuma vide Assalii. Menn zur Assahimasse gekochte Manihotoder ('araknollen bis zur' steifen Masse zugefügt, eine beliebte Speise in den Nordstaatell.

('aitá. C'anna aurantiaral Rose. C'annalceate. Wurzel Arznei. Samen als Sichinuck.

l'a ité assú. Grosse ('. Alpinia ratcemosal Vell. Zingiberareare. Wurzel Volksmittel.

l'aiuia. C'entronia crispifolia Fr. Allem. Melastomaceate. Holz.

r'a iuia-assú. Lobelia villosulus Pohl. Lobeliareate. Blätter Volksmittel.

I'a iu a bravo. Wilde C'. vide ('aiuia.

l'aiuia manso. Zahme ('. Centronia tinctoria Fr. All. Melastomareas. Rinde zum Färben baumwollener 'Leuge.

('aiuia do mato. Wilde ('. Graefenrieda macrophylla Fr. All. Melastomaceae.

C'aiuia vermelha. Rothe ('. Calyrogonimm punctatum Fr. All. Melastomaceare.

Caiuinha. Kleine C. Dichorisandrä proceral Mart. Commelinaceate. Pflanze Volksmittel.

('a ixau vide Jequitiba rosa.

('a ixeta. Tabebuia oltusifolial Bur. Bignoniareene. Holz und Blätter. C'aixeta vide Paú de tucano.

Ca a a muru. Solanum saponaceum Dun. Solanareas. Wurzel statt Radix saponariae.

Cajaty. Ardendron snaveolens Nees. Liaurareate. Holz.

C'ajarana. Schinopsis brasiliensis Engl. Anacardiaceate. Blättel und Frïclite.

('ajerana vide Canjerana.

c'ajuada. Limonade rom Fruchtsafte der cajútrucht.

C'a jú-apeba vide Grỡo de gallo.

C'agazeiro. Cajabaum vide Acaia.

Cajazeiro assu. Grosser Cajabaum vide Acaya-assu.

Cajazeiro bravo. Wilder Cajabaum. Onratea salicifolia st. Hil.

Ochnaceae. Holz. 
Cajazeiro manga. Spondias ruleis Forsh. Luarardiarear. Frucht essbar.

lajazero do mato. Wallajabamm. Talpirial Perkoltiallal Engl. Anacarliacene. Bamhol\%. Frichte Vogelfuss. Niigespaine Volksmittel. Rinde adstringens, enthailt rystallisirtes Tapiririn.

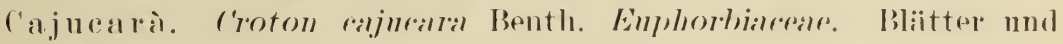
Wurzel.

l'ajueiro. l'ajùbaum vicle Acajù.

Cajueiro ba nana. Bananencajùbanm. Varfetät von Acajù. inclem

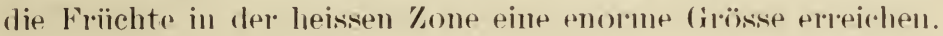

lajueiro bravo. Wilcler l'ajùbanm. Laletia ralophrilal Eichl. Bixaceae, vicle auch siambaiba.

r'ajueiro bravo ia serra. Willer (iebirgst l'. P'inzonil roriarral Mart. et \%uce. Dilleniaceas. Holz.

l'ajueiro do campo. Steppen Cajiubaum vide Acajì-y.

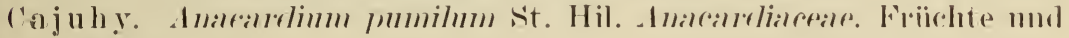
IVwrel.

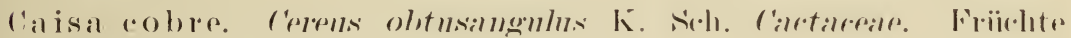
essbar.

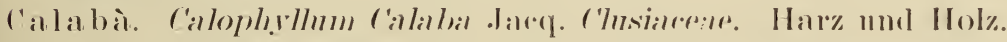

('al la bura vide Mahahujó.

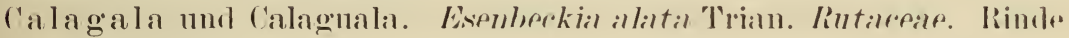
und Hol\%.

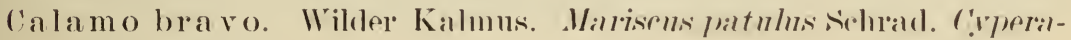
reas. Wurzel.

Calamo da terra. Einheimischer Kalmus. Hypophornm mutans. X. ab. E. Cyperareare. Wurzel.

lalsas da velha. Altweiberhosen. Inuldleia stachioirles l'h. et Schl. und B. brasiliensis .Jaeq. Fil. Srelolumlariareale. Blaittel. officinell.

Caluga. Gilibertia cuneata. E. March. Heleraceate. Wurzel.

('alumba. Simaba salubris Engl. und S. glandulifera Gardn. Simarubaceas. Rinde und Wurzel sehr bitter, officinell.

Calumbi. Mimos? reptans Benth. Mimosaceap. Bliitter.

Cal umi vide Sensitiva.

Calunga. Simaba ferrnginea St. Hil. Simarnhareae. Rinde und Wurzel Volksmittel.

Calunga do campo. Steppen (:. Simalat suflintirosal Engl. Simarubareae. 
Calunga cipo. Calungaliane. Aristolochia cynanchifolia Mart. et Zuce. Aristolochiaceae. Schlangenantidot, ete.

Camaçari und Camaçari vermelho. Falscher Tamakoarè. Caraipa gr Indiflora Mart. Ternstroemiaceae. Gummi und Holz.

Ca ma mì. Aspidosperma nobile Mï̈ll. Arg. Apocynareap. Holz und Milch.

Camandatuba mirim. Hirtflla bracteata Mart. Rosaceae. Frucht und Rinde.

Ca ma p ù. Physalis angulata L. und $P$. brasiliensis Sendt. $P$. viscosa

L. Solanaceae, vide auch Casca l'anta. Früchte essbar. Blätter Heilmittel.

Ca marà. Acroclidium camará Schomb. Lauraceae. Rinde u. Bauholz. Ca mar̀̀-assu. Conepia grandiflora Bth. Rosaceae. Bauholz.

Camarà de bibro vide Pau Pereira.

Camarà branca vide Craveira do campo.

Ca ma rà de boi. Ochsen C. Ternonia helophila Mart. Compositae. Futter für Rindvieh.

Camara bravo. Wilde C. vide Pan Pereira.

Ca ma rà de capaeira. Verbena bonariensis L. Verbenaceae. Volksmittel.

Camarì de carallo. Pierde C. vide Andrequice.

Cam a rà de chumbo. Blei C. zufolge der Früchte vide Camarà-juba. Camarà de folha grande. Grossblättrige C. vide Camarà-juba.

Camarà-japo. Hyptis fasciculata Benth. Labiatae. Blätter aromaticum.

Camarà-juba. Lantana camara L. Verbenaceae. Bliithen und Blätter für Husten.

Camara-juba a marella. Lantana mixta L. Verbenareae. Blätter Volksmittel.

Camarà de leite. Milch C. Vernonia fruticulosa Mart. Compositae. Blätter.

Camarà do mato. Leandra seabra DC. Melastomaceap. Blätter und Rinde.

Ca marà roxa. Lantana lilacina. Verbenaceae. Blüthen.

Camarà do šul. Moquinia polymorpha DC. Compositae. Blätter. Camara-tinga. Weisse (. Lantana hrasiliensis Link. Terbenaceae. Blïtter off.

C'a ma ra ubaia. Jussialea octonervia Lam. Onagraceae. Blätter. ('a ma ra nba ia-tinga. Jussiaea pilosa Rth. Onagraceae. Blätter. 
Cam arão. Krabbenkraut. Arabidata corymbitera Bur. Bignoniaceae. Blïtter und Wurzel.

Ca ma rasinha vide Camarà-juba.

Camarinhos. Eupatorinm orloratnm Link. C'ompositae. Blïthen und Bliatter.

Camarì. Physalis pubescens L. Solanaceae. Frucht essbar.

Cam a ì-l'ana. Falsche C. vide c'ouve de mato.

('a m baiba vide Cajueiro braro.

Camba rà vide Camarà.

Cambarà cipo. Cambaraliane. C'hrysanthemum scandens Vell. Compositie. Blätter.

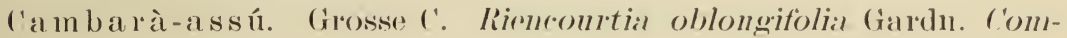
positue. Blïtter.

Cambar à legitima. Jechte C. Moquinial Ciardneri Bak. Com positae. Blätter, benutzt zur Bereitung einer viel verkauten Patentar\%nei fiir Husten ete.

Cambaratuba. Cratylia hylargyrar Mart. Papilionaceate. Rinde. rambica. Speise von der Fruchtpulpe der Brysonima verlatscifolial Rich. mit Mileh und Zucker.

Camboatá und Cambuatá. Ginarea excelsal Bth. Merliacere. Rinde und Bauhol\%.

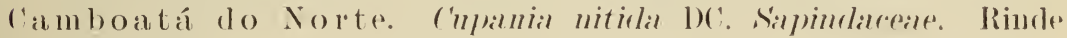
und Hol\%.

('amboint vide Acambuy, heisst anch l'amboim rasteiro. Kripchendes ('.

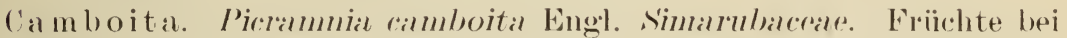
intermitens; frisch enthalten 2.843 Proc. krystall. 'Taririnsiiure. Vorzügliches Bauholz.

Camboita mirim. Kleine C ride Carobinha.

Can buca. Myrciaria plicato-rostata Bg. Myrtareate. Delikate Frucht. Cambuca do mato. Rubarlial glomeratal Bg. Mrrtaceale. Frucht essbar. Bauliolz.

Cambuci und Cambú-y vide Acambuy.

Cambuhy. Engenia pisiformis Camb. Myrtareale. Blaitter, Rinde, Holz.

Cambui vide Aroeira vermelha.

Ca mbu queira vide Abobreira grande; das (iemüse der Blattknospen wird Cambuquira benannt.

Cam butà vide Camboità. 
l'a melaú vicle l'apim côco.

l'a mendatuba mirim vide Camandatuba mirim.

famgaba und Camgamba. Brunfelsia ramosissimal Bth. var. laxiflora Schmdt. Scrophularinaceae. Blätter und Wurzel.

Camisa de Venus. Venushemde. Caladinm bicolor var. Tersrhaftii

Vin. Araceate. Knolle off. Zierpflanze.

('a m unda-y. Kleine Essbohne, wenn gekocht, von P'haseolus nallal

L. Papilionarease.

Camussim. C'araipal grandifolia Mart. Termstroemiaceate. Holz und Gummi.

Can a m ba ia. Rhipsalis phrllanthus K. sch. Cactaceate. Otficinell. l'ananga. Moristical macrophylla Kth. Mrristicaceae. Frichte, Rinde und Holz.

Candîa. Leuchte. Luchnophoral aricoicles Mart. Compositale; im

staate Ilagoas ist es Hirtella ameriallal Aubl. var. gracilipes

Hook. Rosalceale.

Candêa de cajú. Lafoensia replicata Pohl. Lythrareate. Kierpflanze. C'an dêa des angue. Blutleuchte. Cladonia sanguinea Mart. Lichenes.

Heilmittel.

Candúa. Spiloma rosenm Mart. Lichenes. Heilmittel und Farbstofi. Canapa-uba vide Mangue branco.

C'a napúr vide Herva moura.

C'andiúba vide Canella amalrella und J'bí.

C'anêla de Ema. Straussenschenkel. T'ellosial mirritimil Vellos.

V. Camrlirla Mikan. V. lanata Pohl. Vellosiareane.

Canella abacate. Ibacatenzimmt. Ivolendron floribundum Meissn.

Lantalceate. Bauhol\%.

Ganella ama rella. Gelber Zimmt. Nertandra nitirlnla Nees. Laluraceae. Bauholz. sp. Gew. 0.744.

Canella a marella de cheiro. Wohlrechender gelber \%. Avdendron tenellum Meissn. Lanraceare. Bauholz.

Canella barauna. Brauner Zimmt. Oreorlaphne spectabilis Meissn.

Lanralcear. Dunkelhoraunes, sehr dauerhattes Bauholz.

Canella batalha. Kampfzimmt. Oreodaphne rigida Meissu. Lauraceae. Da das sehr harte Holz beim Fïllen sehr Widerstand leistet.

Canella batata. Kartofifelzimmt. C'ordiada trichotoma Vellos.

Cordiaceae? Bauholz. Wurzeln enden mit knolliger Verdickung. Canella bibir ù vide Bibirù. 
(anella branca. Weissel \%. Oreodalphne rassirameal Meissll.

Lauratear. Banhol\%. Spec. (iew. 0.824.

fanella branca do brejo oler da rargem. Weisser sumpt-oder

Thal \%. Nectandra lencothyrsus Meissn. Lanmacae. Bauholz.

Canella brava. Wilder Kimmt, virle Craveiro do mato.

('anella do brejo. Sumpfrimmt. Talamma ovata St. Hil. Magno-

liaceare. Blitter Heilnittel. Bauholz; als Kierbaum angepflanzt.

('anella burra. Gellkistenzinmt, vide Isomo de Parana.

(ianella caixete. Kistenzimmt. Lydendron camellil Meissu. Lall-

raceale. Bauliol\%, bes. zu Kisten.

Canellar capitão môr vicle Canella ferlorenta.

Canella de capoeira vide c'arvallo d'agua.

fanella de cheiro. Wohlriechender \%. Mespilodiphne opiferal Meissn.

Lantareate. Blätter und Rinde officinell. Bauholz. Spec. Gew 0.870.

Ganella fedorenta. Stinkender Kinmt. Nectindral morianthal

Meissn. Lanraceate. Bliithen, Blatter, Rinde, besonders Hol\% be-

sitzen Kohlgeruch. Gutes Bauhol\%.

('anella de folha larga. Breitblätriger \%. Nectandra polyphylla

Nees. Lanlizceate. Bauhol\%.

l'anella de folha miuda. Kleinblätriger 'L. Nectandra hirsuta

Nees. und Croeppertia Cantagallana Meissn. Lanraceae. Bauholz.

('anella funcho. Fenchelzimnt. Mespilodaphne sassafiaz Meissn.

Lanrarceate. Rinde und Holz Heilmittel. Bauholz und Möbelholz.

C'anella garauna vile Cimella barauna.

Canella goiaba vide Canella parda.

Canella gosmenta vide Paú de guiabo.

Canella gua rubú vide Canella parda.

Canella in baiba vide Canella preta.

Canella da India. Cinnamomnm ceylanticum F. Nees. Lalnracear. Canella limão und Canella mirim vide Bibirù.

Canella do mato vide Anhui-pitanga.

Canella mescia vide Canella barauna.

Canella mirim. Oreodaphne odorata Meissn. Lanraceae. Blüthen,

Rinde und Holz.

('anella parda. Brauner \%. Mespilodaphne organensis Meissn.

Lauraceae. Bauholz.

Canella preta. Schwarzer \%. Nectandra mollis Nees. und N. vaga var. vulgaris Meissn. Lauraceae. Möbel- und Bauholz. 
Canella preta da serra. Sthwarzer Gebirgsimmt. Nectandra amara Meissn. Liruraceae. Bittere Rinde offleinell. Vorzügliches Möbelholz.

C'anella puante. Stinkzimmt, vide Canclla félorentil.

Canella santa. Heiliger Z. Fochysia laurifolia Warm. Vochysiacee. Gummi und Bauholz.

( a anella sassafraz vide ('anella funcho.

('a nella sassafraz da serra. Gebirgs sassafras. Mespilodaphur indecora Meissn. Laurarear. Rinde und Sïgespäne officinell. Vorzügliches Bauholiz.

C'anella sebo vide Louro de Paraná.

C'anella secca. Trockener \%. Nectandra lenrantha Nees. Lauraceip. Bauholz.

Canella da serra. Gebirgszimmt. Oreodaphne velutina Nees. Lauraceue. Bauholz.

Ca nella a tôa. Nutzloser '/immt, vide Irvore d'alho.

lanella de veado. Rehzimmt. Lctinostemum lanceolatum Sald.

Euphorbiaceae. Milch und Bauholz.

C'anella de vea do branco. Weisser Rehzimmt. Catesarial brasiliensis

E:chl. Bixaceae. Rinde Heilmittel. Bauholz.

fanella de reado bravo. Wilder R. C'aesaria rupestris Eichl. Bixaceze. Bauholz.

Canella de veado manso. Zahmer R. Eugenia rigida DC. Myrtaceae. Vorzïgliches Bauholz.

Canella do veado do mato. Amphirrhox longifolia sipreng.

Violacease. Rinde und Holz.

Canellinha. Kleiner Zimmit. Mespilodaphne pretiosa Meissn. Lauräreat?. Iromat. Blätter, Rinde und Holz Heilmittel.

C'a nema vide Coerana do Rio de Janeiro.

' 'ang'ua vide Cipo do gôta.

Canida. Licania microcarpa Hook. Fil. Rosalceate. Rinde.

C'aninana ride Cainana.

Canjerana. Cabralea canjerana sald. Meliareate. Früchte und Holz. Canjerana grande. Cabralea pilosa var. glabior DC. Meliacear.

Früchte, Bauholz.

Canjerana da serra. Lucuma : Sapotaceae. Frïchte essbar. Bauholz.

Canjerana miuda vide Issafroeira. 
Canjica. Entschälte Maiskörner, wenn dieselben grobgestossen heissen :

Canjiquinlia.

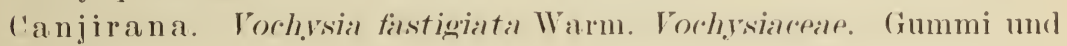
Hol\%.

Canna de assucar. Zuckerrohr. Sirchamm offirinarum L. Gramineae.

Canna brava. Wildes Rohr. Gymerimm parviflorum Nees. Gramineae. Rohr zu P'feilen, etc.

Canna brava assì. Grosses wildes Rohr. Erianthus sacrharoides Michx. var. Mirhanxii Hook. Gramineils. IBlïthenrispe zu Bonquets.

Canna de brejo. Sumpfrolır. $\left\{\begin{array}{l}\text { Caladinm striapes Schott. Araceas. } \\ \text { Heilmittel. } \\ \text { Costus igmens N. C. B. Zingiberareaf. } \\ \text { Samen und Wurzel. }\end{array}\right.$

Canna do campo. Steppenrolu. Ginerimm argentenm $\mathrm{N}$ ab $\mathrm{E}$. Graminease. Rohr zu Pfeile. 'Vierpflanze. Wrurzel Heilmittel.

Canna fistula. Röhrenkassie. Cassia gramlis Linn. Fil. Carsalpinieas. Frucht und Hol\%.

Canna fistula do brejo. Sumpirölurenkassie. Cassial sp'endens Vog. Caesalpinieae. Frucht und Rinde officinell, enthält Chrysophansäure. Bauhol\%.

Canna fistula merun. Kleine Röhrenkassie. Cassie ferruginea Schrad. Cassalpiniear. Frucht und Holz.

Ca un a flexa. Pfeilrohr vide Canna do campo.

Canna imbé. Dieffenbarhial seguine Schott. Araceap. IBlitter Heilmittel.

Canna de macaco. Iffenrohr. $\left\{\begin{array}{c}\text { Dichorisandral thyrsifolia Mik. ('om- } \\ \text { melinaceae. Heilmittel. } \\ \text { Costus discolor Rosc. Zingiberacear. } \\ \text { Wurzel. }\end{array}\right.$

Canna do mato vide Paco-caa-tinga.

Canna mepique. Scharfschneidiges Rohrgras am Amazonenstrom. Gramineae.

Canna de passarin ho. Vogelrohr. Panicum latifolinm L. Gramineas. Futterpflanze.

Canna do reino. Arundo Donax L. Gramineas.

Canna do rio. Flussrohr vide Paco-caa-tinga. 
Canna roxa do brejo. Rothes Sumpfrohr. Costus spiralis Rose. Zingiberaceae. Wurzel.

ranua de ribora. Schlangenrohr. Kunthia montana H. B. Kth.

Palmae. Schlangenanticlot.

C'annarana und Cannarona. Falsches Rohr. Paspalum pusil-

lum Vent. Gramineae. Futterpflanze.

Canninha. schwacher Zuckerrohr-Brandwein.

C'an oés vide Mangua scriba.

Canopy und Canopy-tree. Melilocea bijuga Jacq. Sapinclaceap.

Heilmittel.

Cansançao. $\left\{\begin{array}{l}\text { Jatropha urens var. osteocarpa Miill. Irg. Eujphor- } \\ \text { liaceae. Blätter brennend. Namen ölreich. } \\ \text { Crera subpeltata Miq. Crticaceas. Brennnessel. }\end{array}\right.$

Cany-caracì. Gegohrenes Getränk der Indianer aus Mandioccawurzel.

C'anudo. $\begin{aligned} & \text { Röhre. Tephrosia leptostachrs DC: var. leptoph.rlli Bth. } \\ & \text { Papilionaceae. Rinde und Blätter Heilmittel. } \\ & \text { vide Algodão sinho. }\end{aligned}$

C'anudo de lago. Seeröhre vide Mat ı cabras.

('anudo de pito. Pfeifenröhre vide sapucainha.

Canudo de pito de leite. Milch Pfeifenröhre. Mabeal fistuligeral

Mart. Euphorbiareae. Milch, Rinde und samen officinell.

Canxim. Martenus horrida Reis. C'elastinareas. Rinde und Blätter.

Canza. Flöte ron Taquararohr.

Canzenze. Pithecolobinm niopoides spr. Mimosaceate. Siamen mul

Rinde.

Cân-hỵ. Weinartiges Getränk aus dem Frnchtfleische der Enterpe edalis.

C'a ponga vide Coerana.

Caoium. Indianerbier ron gekochtem und gekautem Mais.

C'apa homem ride Cipó imleé.

Cа ра̃o. Waldinseln auf dem Camposgebiete. corrumpirtes Tuprwort ron Caa-Mald. paim-isolirt.

Capano bravo. Wilder C. Rhopala romplicata H. B. Kth. Proteacere. Rinde.

Caparosa. Vitriol. Jussaea raparosa Camb. Onagraceae. Blätter zum Färben.

Caparosa do campo. Nteppenvitriol. Neer theifera Oerst. Nurtaginaceae. Zum Färben. 


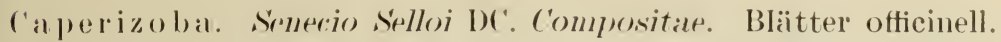

Caperizoha branca. Weisse ('.

f Senerio brasiliensis Less.

Caperizoba rheirosa. Wohlriechende C.l var. tripitrtitus Bak.

C'ompositale. P'flanze officinell. Aeth. Oel.

('aperizoba vermellat. Rothe ('. Erechthites valerianafofolia I)(. var. organensis Bak. P'flanze Heilmittel.

Capetizoba vicle Herva de bicho.

Capim. Gras, von Tupi; car-Blatt; pyim-klein.

Capim d'agua. Wassergras. Panicum alnessum Lam. Gramineae.

Viehfutter.

Capim am a lososo. Bitteres Gras. Palplophornm mucronulatum

N. ab E. Grammintere. As Heilmittel.

Capim andaca. Panicnm tricanthnm N. ab E. Gramineae. Viehfutter.

Capim andrequeci. Ichnanthus bambmsiflorns Döll. Ciramineate. Vielifutter.

l'apim de Angola. I'anirnm spectalile N. ab E. Gramineare. Futtergrass kultivirt.

l'apim arroz. vide c'apim puba.

Capim assí. Grosses Gras. Sorghmm minarnm Hack. firamineale. Eragrost is bahiensis Schult. (Viehfutter.

Capim atana. Erogrostis pilosal ['. de Beaur. Gramineare. Heilinittel.

Capim balsa. Paspalnm riparinm X. ab E. Giamineae. Viehfutter.

Capim bamló. (Jøral Horilmula Rarld. Gramineare. Viehfutter und Zierpflanze.

Capim bambú sinho. Olyral bolyzorlionles Frin. Gramineas. Viehfutter und /ierpflanze.

Capin barba de bode. Ziegenbart. Tylothrastal petrosal Iöll. Gramineila. Heilmittel.

Capim Bengala. Eragrostis intermpta Lam. Gramineae. Kultivirtes Viehfutter.

Capim de bezerro. Kälbergras. Panirnm compostum L. Gramineare. Viehfutter.

Capim brilhante. Lageno rarpus alamantinus $\mathrm{X}$. ab E. C'iperacear. Pflanze, welche auf Diamanten-haltigem 1)istrikt vorkommt. Capim bucha. Gewehrproptengras. Leptochloa srabra. X. ab E. Gramineate. 
Capim de burro. Eselsgras. Paspalum fasciculatmm Willd. Graminear. Futtergras.

Capim de camellao. Kyllingia pungens Lind. Cyperaceat. Aromatische Wurzel officinell.

('apim canela de Ema. Saccharmm holcoides Hack. Gramineae. Blüthenähre zu Bouquets. Wurzel Heilmittel.

C'apim de cheiro. Wohlriechendes Gras. Andropogon reriferus

Hack. Gramineate. Blätter wohlriechend, Heilmittel.

Capim cheiroso. Kyllingia olorata Vahl. Cyperareate. Blätter

Heilmittel, liefern 0.15 Procent aeth. Oel.

Capim cidrilho und Capim catinga. Andropogon densifforus

Steud. Gramineare. Heilmittel.

Capim de Colonia. Koloniegras. Paspalum densum Poir. Gramineae.

Panicum maximum Jacq. Kultivirtes Viehfutter.

Capim commun, vide Pé de galinha.

Capim de galinha. Hühnergras. Poa annua L. Gramineae. Futter.

Capim de estrella. Sterngras. Paspalum stellatum. Fluegg.

Gramineate.

Capim de fogo. Feuergras. Vilea arguta Nees ab E. Graminear. Brennmaterial.

C'apion de frechas. Pfeilgras. Tristachya leiostarhya N. ab E. Gramineate.

('a pim Frei Luiz vide Capim melarlo.

Capim gengibre. Ingwergras. I'arialla zingiberina Iöll. Gramineae. Samen als Heilmittel.

Capim gengibre burro vide Capim burro.

Capion gengibre rasteiro. Paspalum fialcatum N. ab E. Gratmineae. Futtergras.

Capim gigante. Riesengras. Panicum megisto: Schult. Gramineat. Futtergras; die alten Blätter zum Poliren.

Capim de Guiné. Guineagras. Panicum jumentorum Pers. Gramineae. Kultivirtes Grass.

rapim jasmin da serra. Eleonarus lilingins Hack. Gramineate.

ITurzel aromatisch.

Capim bixa. Polirgras, vide Capim gigante. 
('apim mato de sapo. Froschhandgras. Paspalum extematum. N. ab E. Gramineate. Viehfutter.

Capim melado. Honiggras. P'anicmm melinis Trin. Gramineate. Kultivirtes Gras.

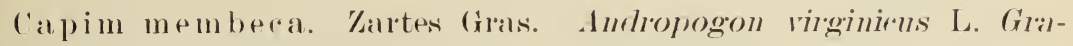
mincile.

Capim mular. Maulthiergras, vide Capim de Colonia.

Capim navalha. Barliermessergras. Hypolitrmm Schrarlerianmm N. al) E. C'rperalreate. Heilmittel.

Capim orvalho. Tangras. Eragrostis rufescems sohult. Graminear. Heilmittel.

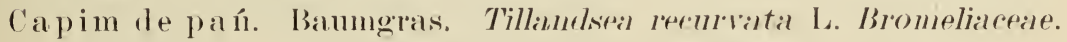
Heilmittel.

Capim de papuan. Kugelgras. Stenotaphrum glabrum 'Trin. var. americantum Döll. Graminear. Wurzel als Ersatz der Rad. graminis.

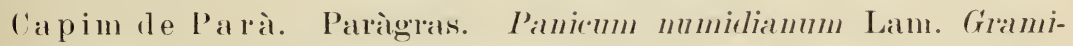
near. Kuntivirtes Futtergras.

Capim de pavao. P’auengras. Panirum crus galli L. val. echinatum Döll. Ciraminturae. Viehfutter.

Capim-peba. Plattes Gras, corrumpirtes Tupiwort von Caa-Blatt, pyim-schmal und apeba-platt. Andropogon bicornis L. var. gemuinus Hack. Gramineate. Wurzel Heilmittel.

l'apinı pé de galinha. Hiihnertussgras. Eleusine indical Gaertu. Gramineate. Unkraut etc.

('apim pé de pavao. Pfauenfussgras vile Capim pavao.

C a pim de Pernambuco vicle Capim de Angola.

Capim picao. Kilettengras, vide Capim pé de galinha.

Capim da planta.| Panicum maximum .Jaeq. Var. gongylodes Döll. Capim da praia. $\int$ Gramineae. Kultivirtes Viehfutter.

Capim-puba. Weiches Gras. Panicnm crus galli L. var. silbulicolum Döll. Granineae. Viehfutter.

Capim rabo de raposo. Fuchsschwanzgras. Panicum scandens Trin. Gramineite. Heilmittel.

Capim rasteiro. Kriechendes Gras. Pasyalum falcatum N. ab. E. Gramineat. Futtergras. 
Capim rei. Königsgras. Alialbodal poarchon Seub. Trridaceae. Wurzel Heilmittel.

Capim da roza. Feldgras. Syartina brasiliensis Radd. Granineae. Viehfutter.

('a pim rosete. Rosettengras. Cenchrus triluloides L. Giranineac. Capin de sapo vide Capim mã de sapo.

Capim taquari. Rohrgras. Panicum sanguinale L. var. longiglume Döll. Gramineae. Viehfutter.

Capim taquarisinho. Kleines Rohrgras. Audropogon spathiftorus Kth. Gramineae. Wurzel Heilmittel.

Capim da tartaruga. Schildkrötengras. Panicum elephantipes N. ab E. Gramineae. Frass der Schildkröten.

Capim-tinga. Weisses Gras. Panicum discolor Trin. Giranineae. Vielıfutter.

Capim ubatim vide Milho bravo.

Capim vassoura. Besengras, vide (aipin-peba.

Capinân. Mẹrtaceate. Frucht essbar.

('api-poatinga. Paepalanthus speciosus Koke. Eriocanlaceare. Heilmittel.

('apitão do mato. ) T'erminalia brasiliensis Camb. C'ombretaceae. Capitão do campo.j Heilmittel.

Capitão de Pernambuco. Hydrorotyle lirta $\mathrm{R}$. Br. var. sessilis

Urb. Cmbelliferae. Heilnittel.

Capitaro sinho vide Ameixeira de espinha.

Capitenga vide Capin millıın.

Capitiú. Mollienrla lanrina Tul. Moninniaceale. Heilnittel.

Capitura. Panicum Beanrepairei Hack. et Glas. Gramineae.

Capivara. Aristolorhia hirostris Duch. Aristolochiaceae. Wurzel officinell.

('a pichingu i. C'roton echinocarluns Mïll. Arg. Euphorbiaceace. Rinde officinell.

Capichingui de sangue. C'roton Horihundus Mart. Euphorlialceate. Rinde liefert blutrothes Kino ähnliches Secret.

l'a poeira branca. Solannm lencadendron Sendt. Solanaceate. Heilmittel. 
Capororoca vide Cia-pororoca.

Capororoca de Alagoas vide Azeitoma do mato.

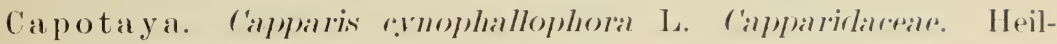
mittel.

Caprenva vide labureiba.

Capueira. Waldnachwuchs, mach Rodung des Trwaldes, Wemn sich schon grössere Bäume gebildet, damn ('apmeirã).

Capupuba vide Capim-peba.

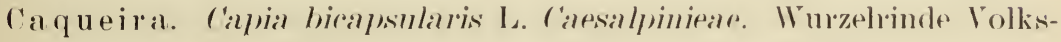
mittel.

Caquibosa vide Guaxima macho.

Carà bedentet in der Tupisprache essbare Knolle.

Carà amarella. (ielbe C. Dioscorea hatatas Dev. Dioscorrarrar. Kultivirt. Proteinstoffe 1.319, Stiirkemehl 14.747 Proc.

('a rà de Angola. Angola ('. Inoscorea vulgaris Miq. Inioseorranreare. Kultivirt, hat Proteinstoffe 1.:386. Stärkemehl.

Carì do ar. Luft C., vide Cara sapateiro.

Car’a assír. Grosse (', vide Cara sylvestre.

Car à barbada. Bärtige (:. Diosrorea dorleranenrar Vell. Iloscoreacep. Kultivirt, hat Proteinstoffe 1.505, Stäremehl 18.460 Proc.

Carà branca. Weisse C. Diosrorea sativa L. Dioscoreareate. Kultivirt, hat Proteinstoffe 3.228 , Stärkemehl 10.410 Proc.

Car ì brava. Wilde C., vide Caratinga brava.

Carà de caboclo. Indianer ('. Bomaria salsilloirles Röm. Alstroemeriaceae. Indianerspeise.

Carà côcô. Cocosnuss C. Diosrorea hastata Vell. Diośrorearear. (Gegessen, doch nicht kultivirt.

Carà doce. Süsse C., vide Cara mimosa.

Carà de folha pintado. Gefleckte Blatt C. Dioscorea rliversifolia Grieseb. Dioscoreaceae. Zierpflanze.

Carà de espinha. Stachel '?, vide Cara Sapateiro.

Carà de Guiné. Guinea C. Diosrorea aculeata L. Mioseorearcale. Proteinstoffe 2.092, Stärkemehl 20.505 Proc.

Car à in hame. Inhame C. Dioscorea alata L. Diosroreareare. Kultivirt, hat Proteinstoffe 1.25, Stärkemehl 14.747 Procent. 
Carà mandiocca. Mandioka C. Dioscorea purpurea Roxb.? Diosroreaceae. Kultivirt.

Carà mandiocea brava. Wilde Mandioka C: Dioscorea farcta

Grieseb. Dioscoreaceae. Wild, wird genossen.

Carà do mato. Wald C. Bomaria spectabilis Śchenck. Alstroemeriaceae. Zierpflanze und Indianerspeise.

Carà mimosa. Zarte C. Dioscorea brasiliensis Willd. Dioscoreacer. Kultivirt, hat Proteinstoffe 1.22, Stärkemehl 7.94 Procent.

Carà de Para. Dioscorea cayennensis Lam. Diosroreaceae. Kultivirt, hat 16 Proc. Stärkemehl.

Carà pé de anta. Tapirfuss C. Dioscorea filiformis Grieseb. Dioscoreaceae. Kultivirt, hat Proteinsubstanzen 2.666, Stairkemehl 10.03 Proc.

Carà cascos. Huf C. Diosconea laxiflora Mart. Dioscoreaceae.

Wild, wird gegessen.

Carà de pelle branca. Weisshäutige C. Dioscorea conferta Vell. IDioscoreaceae. Mit rothen pelionla als C. de pelle vermelha, eine Varietät. Beide werden kultivirt. Proteinsubstanzen 4.379, Stärkemehl 26.059 Procent.

('a rà s a pa te i ro. Schuster C. Dioscorea bulbiferal L. Dioscoreaceap. Kultivirt.

Luftknollen haben Proteinstoffe 1.834, Stärkemehl 14.860 Proc. Erdknollen " " * " $\quad 1.415, \quad$ "

Carà sem bar ba. $\left\{\begin{array}{c}\text { Bartloser C. Dioscorea subhastata Vellos. Dios- } \\ \text { coreaceate. } \\ \text { Dioscorea piperifolia Willd. var. legitima Grieseb. } \\ \text { Dioscoreaceae. Kultivirt, hat Proteinstoffe } \\ 2.27, \text { Stärkemehl 18.21 Proc. }\end{array}\right.$

Ca rà sylvestre. Wilde C. I Rajania brasiliensis Grieseb. Liliaceae. Ca rà salza. Sassaparill C. I Wurzel Heilmittel.

Car à na mby. Ohren C. INioscorea adenorarpa Mart. Dioscoreaceas.

IVild, wird gegessen.

Car à-ch ichú vide Herva moira.

C'aracol. Phaseolus caracalla L. Papilionaceae. Kultivirte Bohne. Caraguatá. Nidularia bracteatum Metz. Bromeliaceae. Zier- und Bastpflanze.

Caraguatá assú. Fourroya cubensis Hak. Agaveae. Bastpflanze. 
Carahia vide Abobora d'agua.

Caraiba. Simaruba rersicolor St. Hil. var. angustifolia Engl. Simarubareae. Heilmittel.

Caraiba do campo vide Páu d'arco do campo.

Caraibo. Licania microcarpa Hook. Rosacear. Heilmittel.

Caraipe. Mornilea utilis Hook. Fil. Rosareare. Heilnittel n. Banluolz. Caraipé das aguas vide Turiuva.

Caraipé vermello. Cydista aequinortilis Miers. Bignoniandeate. Heilmittel. Heisst auch Caraipi.

Cara-jurì. Astroemeria montirnlal Mart. Astroemerialeale. Wur\%el dient den Indianern zur Nahrung.

Ca r a-jur ì vide auch rarapitaia.

('a ra jù ru. Arrabirlapa Chiral Verl. Bignoniareate. Rothen Farbstoff liefer'nd.

Carambola. Arerrhoa carambola L. Oxalirleae. Kultivirt. Friichte essibar.

Ca ramburì. Erfrischendes Getrïnk aus Maisabkochumg.

Caraná vide Muhi.

Caraná vide Jará und Caranai.

Caraná do Río Negro vide Cahuaia.

Caranai. Manritia aculeata H. B. Kth. Palmae.

Caranai do mato. Lepiclocarymm teme Mart. Palmate.

Carana-i. Mauritia pumila Mart. Palmap.

Carana ú ve vicle Carnauba.

Carandá. Trithrinax brasiliensis Mart. Palmae.

Carandai. Copernicia rerifera H. B. Kth. Palmae.

Carangé-catá vide Gravata do mato.

Caranná. Protimm rarana Mar.h. Burserareae. Balsam, Harz und Bauholz.

Carapá. Carapa guianensis Aubl. Meliaceae. Oelreiche Samen, Rinde und Holz.

Carapacú-peteca vide Herva de veado.

Carapana-assù. Grosser C. ) Bauhölzer in Pará, noch nicht beCarapana mirim. Kleiner C. $\}$ stimmt.

Carapato. Desmodium barbatum Bth. Papilionaceae. Homeopatisches Heilmittel. 
Carapeirana vide Turiuva.

Carapepe vide Abobora d'agua.

Carapiá. Dorstenia multiformis Miq. Lrtiraceae. Wurzel officinell. Carapiá in Ceará vide Apihi.

Carapiá in Rio Grande do śul. Dorstenia arifolia Lam. var. pinnatifida. Vrticaceae. Wurzel officinell.

Carapitaia. Carlotea formosissima Arrud. Amarvllidareae. Zwiebel Heilmittel.

Carapuça de marinheiro. Matrosenmiitze. Friflericia speciosa Mart. Bignoniaceae.

Ca ra tinga. Dioscorea piperifolia „; triangularis Willd. Dioscoreacex.

Wild. Wird genossen.

Caratinga brava. Wilde Caratinga. Dioscorea sinuata Vellos.

Dioscoreaceae. Wird genossen. Proteinsubstanzen 2.318, Stärkemehl 8.0 Procent.

Caraúba vide Caraiba.

C.a raurá vide Caróa.

Caray po. Coleophora gemmiflora Miers. Thymeliaceae. Heilmittel. Cardamomo do mato vide Assuceno do brejo.

Cardamomo sylvestre. Alpinia nutans Rose. Zingiberaceap.

Wurzel und Samen.

Cardo und Cardo anano. Cerens setarens Salm lyy. Cactaceap. Cardo amarello vide Argrimonda.

Cardo Coceta. Cereus macrogonus Salm Dyk. Cactaceae.

Cardo bravo. Wilde Distel. Sil.rbum marianum Gaertn. Compositre. Heilmittel.

Cardo de Santa Helena. Ilanthinm spinosum L. Compositae.

Cardo santo. Heilige Distel. Centaurea ralcitropa I. C'ompositae. Cardo santo am arello vide Agrimonda.

C'arerú vide Carurú.

Caribé. Getränk von in Wasser gelïsten Beijúkuchen; ferner eine Speise von der Fruchtpulpe der Abacate.

Carimân. Speise aus der in Wasser eingeweichten Mandioccawurzel. Carimô. Frisches, noch feuchtes Mandioccastärkemehl.

Carnauba. Wachspalme. Copernicia rerifera Mart. Palmae. Wachs der Blätter, Friichte, Stamm und ITurzel. 
rarne de varca. Kuhfleisch. Rhopalia elegans Schott. Protealrear. Bauholz.

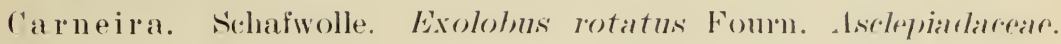

Frucht und Blätel.

Carnicula. Mimosal polyclartyla Hb. et 13. Mimosareaf. Heilmittel. Carôa. Neoglazioria rariegata Metz. Bromcliareare. Faserstoff- mur Zierpflanze.

Caroarí-cá. P’iperomia hederaceal Miq. P'iperareale. Heilnittel. Ca roba. I Jararanda brasilianal Pers. I Bignoniareale.

Ca roba. ¡ Jacaranda subrhombea $\Lambda$. I)C. I Blätter antisyphiliticum. Caroba-assì. Gousse ('., vide Copraia.

Caroba brancar vide Cinco follas.

Caroba do campo. Steppen ('. Jalrallancla rufil Mans.; Jalcaranta paucifoliata Mart. Bignoniareale. Antisyphiliticum.

Caroba falsa. Falsche C. Memoral glaberima K.Soh. Bigmoniarear. Heilınittel.

Caroba de flor verde. Grïnblühige C. Colostas antiśryllilitiral

Mart. Bignoniareae. Intisyphiliticum.

Caroba guyra. Vogel ('. Bignonia purgans Ried. Bignoniareale. Abfïhrmittel.

Ca roba do mato. Wilde C. .acaranda micrantha Cham.; .Jacaranda semiserrata Cham. Bignoniareate. Antisyphiliticum.

Caroba miriun. Kleine C. .Jacaranda macrantha Cham.; Jacaranda puberula Cham. Bignoniaceae. Antisyphiliticum.

Caroba miuda. Kleine C., vide Caroba mirim.

Caroba preta. Schwarze C. Jacaranda obovata R. Br. Bignoniaceae. Vorzügliches antisyphiliticum. Enthält Carobin cryst. und Acid. carobinicum eryst.

Caroba salsa. Sassaparill C. Jacarancla cuspiclifolia Mart. Wurzel und Blätter Ersatz des Sassaparille.

Carobinha. Kleinblättrige C. Jacaranda caroba R. DC. Bignoniareae. Kräftiges antisyphiliticum.

Carobinha do campo. Steppen C. Jacalandi oxyphilla Cham.

Bignoniaceae. Gleiche Benutzung.

Carolina. Adenanthera pavonina L. Mimosareae. Samen als Schmuck. Blätter, Rinde und Wurzel Heilmittel.

Carqueija amargosa. Bittere ('. Barcharis genistelloirles Pers. Compositae. Heilmittel. 
Carqueija amargosa do campo. Steppen C. Baccharis stenorephala Bak.; Baccharis genistelloides Pers. var. trimera Bak. Compositae. Heilmittel.

Carqueija doce. Süsse C. Baccharis articulata Pers. var. Ganrlichiana Bak. Compositae.

Carrapateira vide Mammono.

Carrapeta vide Assafrôa.

Carrapichinho. Kleine Klette. Trinmfetta longicoma St. Hil. Tiliacere.

('a r'rapicho. Klette. Birlens pilosus L. Compositap.

Zornia diphylla Pers. Papilionaceap.

Urena sinuata L. Malvaceae. Die Brasilianer benennen alle Pflanzen, deren Frïchte, Ilätter etc. an den Kleidern festhaften: carrapicho.

Trinmfetta longicoma st. Hil. Tiliaceae.

Triumfetta heterophylla Lam. , Tiliaceap.

Triumfetta longicoma St. Hil. I Faserstoff.

Carrapicho de agulha. Nadelklette. Biclens chrysanthemoirles Michx. Compositae.

Carrapich o beiço do boi. Ochsenlippenklette. vide Arruda campina.

C'arrapicho de boi. Ochsenklette. Trinmfetta eriocarpa St. Hil.;

Triumfetta rhomboidea Jacq. Tiliaceae. Faserstoff und Heilmittel.

Carrapicho de calçada. Wegeklette. Triumfetta sepium St. Hil.;

Triumfetta semitriloba L. Tiliaceas. Vorzïgliche Faser.

Carrapicho do campo. Steppenklette. Barcharis tarchonan-

thoides DC. Compositale.

Carrapicho cheiroso. Riechende Klette. Acanthospermum hirsutum DC: Acanthospermum xantloirles DC. Compositae. Wurzel Heilnittel.

Carrapichocarapato. Zeckenklette. Desmodium barbatum Benth. Papilionaceae. Als Herlysarum Ildefonsii homeopathisches Heilmittel.

Carrapicho de cavallo. Pferdeklette. Trinmfetta nemoralis St. Hil. Tiliaceae. Faserstoff.

Carrapicho de Cearà. Krameria tomentosa St. Hil. Polygalacer. ITurzel officinell.

Carrapicho grande. Grosse Klette. Mimosa Velloziana var. glabra Mart. Mimosaceae. Blätter. 
Carrapicho miudo. Kleine Klette. Trinmfetta ralicnlata Vell. Tiliaceae. Heilnittel.

Carrapicho rasteiro. Kriechencle Klette. IJesunorlinm triflormm DC. Papilionaceae. Heilmittel.

Carrapicho do sertao. Wiistenklette. Irhthrothere hirsnta Garr.

Compositar.

Carrapicho vassouras. Besenklette, vide Carapato.

Garapicho vermelho. Rothe Klette. Cosmos ramlatus HBKth.

Compositile. Heimittel.

Carrasco. Steppenwald mit zwergartigen Biiumen.

Ca rrascó. Cambessidesia rorrmbosa I)C. Melastomareare. Heilmittel. Carrascó do campo vicle ('arrapicho do campo.

Caruá-iba und Curuá-iba. l'ordia excelsa 1. DC. Cordiareae.

Rinde offic.nell, enthält krystallinisches ('ordianiu. Bauhol\%.

Ca rum-bam-bamba vile Rutin.

Caruto. Genipa caruto HBkth. Rubiaceae. Frucht, Rinde, Holz. Carvalho. Eiche (bras.) Rhopala lucens Meissn. Rhopala heterophylla Pohl. Rhopala Gardneri Meissu. Rhopala longepetiolata Pohl. Rhopala brasiliensis Kl. Proteareae. Banholz.

Carvalho d'ag'ua. Wasserreiche Inrlripetalum multiflornm Schott.

Proteaceae. Bauholz.

Carvalho secco. Trockene Eiche. Irrlentron Horihundum Meissn.

Lanrareae. Bauholz.

('arvalho da serra. Gebirgseiche. Rhoplalir P'ohlii Meissn. Proteaceae. Bauholz.

Carvalho do sertao. Rhopala Martii Meissn. Proteaceae. Bauholz. Carvalho vermello. Rothe Eiche. Popppigia procera Presl. var. conferta Bth. Caesalpiniaceap. Vorzügl. Bauholz.

Carvoeira. Kohlenbaum. Callisthene maior Mart. var. pilosa

Warm. Tochysiaceae. Kohlen zu Sprengpulver.

Carurú (von caa - Blatt, rerú - essbar). Euxolns viridis Moq. Amaranthareae. Gemiise.

Carurín d'agua. Wasserspinat. Neptunia oleraceat Lour. Mimosaceae. Gemüse.

Carurú a marello. Gelber Spinat. Amaranthus flavns L. Amaranthaceae. Gemüse.

Carurú amargoso. Bitterer Spinat, vide Caperizoba vermelha.

Carurú assì. Grosser Spinat. Phytolacca thyrsifolia Fenzl. Phytolaccaceae. Gemüse und Heilmittel. 
Carurú azedo. Saure Spinat. Hibisens sabdariffa L. Malvaceae. Gemiise.

Caruró bahiana. C'orchorns olerarens L. Tiliareae. Ciemuise und Faserstoff.

Carurú bravo. Wilder spinat. ('entropogon smrinamensis Presl.

Lobeliaceate. Wird von den Indianern genossen.

C'a rurú guassì. Grosser Spinat. Phytolacer decandra I. Phytolaccaceae. Gemüise, Farbstoff und Heilmittel.

Carurú miudo. Kleiner Spinat. Enxolns olerarens Miq. Amaranthaceare. Gemiise.

Carurú perdrix. Rebhuhnspinat, vide Carurú guassì.

Carurú preto. Śchwarzer Spinat, vide $\Lambda \mathrm{g} u$ arà-qui ya.

Carurú de sal vide Herva de sal.

Carurú de sapo. Froschspinat. Oxalis Martiana Zucc. Oxalidacer. Carurú selvagem vide Carurú assù.

Carurú de vea do. Rehspinat. Solannm inapquale Vellos. Solanaceap Heilmittel.

Carurú verde. Griiner Spinat. Enxolus ranrlatum Miq. Amaranthaceae. Gemiise.

Carvao branco. Weisse Kohle. Bauholz in Matto Grosso.

Casca d'Anta. Tapirrinde. Irvmis Winteri Forst. var. reroluta.

Winteraceae. Offlcinell.

Casca d'anta brava. Rauwolfiia Bahiensis A. DC. Iporvnarear. Heilmittel.

Casca branca. Weisse Rinde, vide Coto-coto.

Casca doce. Siisse Rinde, vide Buranhem.

Casca doce do mato. Wilde süsse Rinde. Andradea dnlcis Fr.

Allem. Nyc aginaceate. Heilmittel und Bauholz.

Casca de empigem. Flechtenrinde. Cybianthus detergens Mart.

Mvrsinaceae. Heilmittel.

Casca de lagarto. Eidechsenrinde, vide Pau de lagarto legitimo. Casca de Maran a vo vide Casca preciosa.

Casca para tudo. Rinde für Alles. Cinnamodendron axillare Endl.

Canellaceas. Hortia arborea Engl. Rutareae. Heilmittel und Bauhölzer.

Casca preciosa. Kostbare Rinde. Mespilorlaphne pretiosa Mart. Lanraceae. Gewürz.

Casca de tatú. Gürtelthierrincle, vide Tatú. 
('ascavel. Klapperschlangenkraut. C'rotillarial striatar D)( I'apilionaceae. Schlangenantirlot. Die schoten machen Geräusch wie Klapperschlange.

Cascavelleira (aus casco-Hornhaut und cavilha-stift). Klapperpflanzen, ebenfalls Antidote. C'rotalarial flil ricomia lith. Crotalaria holosericeal v. griseal Mart. Crotalirria andgyroirles HBKth. Crotalar a sublecurrens Mart. I'apjilionalerare.

Casco de cavallo. Pferdehuf. Sacarandir molnlata steud. Bignomiaceare. Heilmittel. Die Form der Frucht.

Cascos. Dioscoreal laxifforal Mart. Dioscoreaceate. Kinolle esshar. Blätter Heilmittel.

Cassatinga de espinlo virle Catota de espinho.

Cassatinga mansa. Zahme ('. Solanum indigoferum St. Hil.

Solanaceace. Farbstoff und. Heilmittel.

Castanha de bugre. Indianerkastanie, viole Jatolà.

Castan ha de Ceari. Pachira alyatioa Aubl. Streuleaceale. Oel-

reiche Samen essbar.

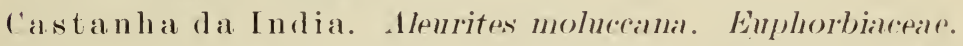

Castanha de Maran häo. I Bertholletia exrelsad Humb. Myrtareare. l'astanha le Parì. I Essbare Samen. Baulolz.

Castanha le purga. P'urgir Kastanie. Ompllalea ciandral L. var.

gennina Miill. Arg. Euphorhiareate. Frïchte abfïhrend.

Catacaem vide Gramixameira.

('atacanhem. Rhopali Sellewii Krr. Proteareate. Bauliolz.

Catağôa und ('atigoa (von ('aa - Blatt, tagôo - blutroth) vide Caatigua und auch Angelim rosa.

('atagua. Metrodorea mbescens St. Hil. Rutaceap. Rinde rothen

Farbstofi. Mangalo hat auch dieselbe Beriennung.

Cataia von caa - Blatt, taia - Peffer. Drymi- Winteri Forst. var. granatensis Winteraceate. Otficinell. Bauholz.

('a fanduba (corrumpirt von Caa-Blatt, van-(lunkel, juba-gelb).

Piptadenia moliniformis Benth. Mimo:alrear. Bauholz.

Cat a ì ba vicle Acatauba.

Caticôa Spiloma rosenm Mart. Lichenes. Rother Farbstoff und Heilmittel.

latimpû́ra. Getränk rer Indianer. Mandioccabier, versüsst mit Honig der II aldbienen.

Catinga (von caa - Blatt, tinga - weiss). Teromal catinga Bur. et K. Sch. Bignoniaceae. Bauholz. 
Catinga de bode. Bocksgeruch. Hypericum brasiliense Chois. Guttiferae. Heilmittel.

Catinga branca. Zinthoxylum rigidum H. et B. Rutaceate. Bauholz. Linharea tinctoreal Arrud. Laurareate. Bauholz und Heimittel.

Catinga de formiga. P'ectis aporlerephlala Bak. ('ompositae. Heilmittel.

Catinga de macaco vicle Gitirana.

Catinga de mulata. Tanacetum balsamitum L. Compositae. Heilmittel.

Catinga de negro. ('teome demdroides schult. Calpparidaceate. Heilmittel.

Catinga de paca. Eliteagnus ratinga Fr. All. Elateanguareate. Heilmittel.

Catinga de poreo. Síxifraga Horibunda HBKth. Saxifiagaceate. Heilmittel.

Cat ingueira. Stinkbaum. C'aesalpinia hracteosa Tul. und C. Gardneriana Benth. Caesalpiniaceale. Bauholz und Heilmittel.

Catingueira brava. Croton mians var. argyrogiossum Müll. Arg. Euphorbiaceale. Heilmittel.

Catingueira do mato. Zanthoxylum amagense Tul. Rutaceae. Bauhol\%.

Catolé vide Caqueiro Catolé.

Catotá. Anreliana lucidal Sendt. Solanaceale. Heilmittel.

Catota de espinho. Solannun hexandrun Vellos. Solanarcale. Heilmittel.

Catuába. Enothoxylum nitirlunn siprue. Erythoxylaceate. Heilmittel. Catuiba vide Acatauba.

Caúa. Abführende Pflanze in ('ear'̀̀.

Cauassú. Threkaldial bracteata Fr. Lll. Salsolareale. Heilmittel.

Ca ú-hy. Prestonia tomentosia. Apocynaceale. Heilmittel.

Cauim. Gegohrenes Getränk der Isdianer von gekochter und gekauter Mandiocea. cauim, cai-Essig, cauim piranga-Rothwein, cauim tatì - Zuckerbranntwein.

Caúna. Bauholz in Santa Catharina.

Caúré. Bauholz in Parà.

Ca ustico vegetal. Acalypha pruriens Nees. et Mart. Euphorbiacere. Ca úvi. Piptadenia colubrina Bth. Minosareae. Blätter Heilmittel.

Rinde zum Gerben. 
Cavallina. Equisetnm pyramidale Goldn. Equisetalceale. Heilnittel. Po'instengel.

Caxaporra de gentio. Terminalial fagifolial Mart. Combretareae. Bauholz und Heilmittel.

Caxaporra do sertaó. Terminalial argentea Mart. et Inec. Combretalorile. Baulol\%.

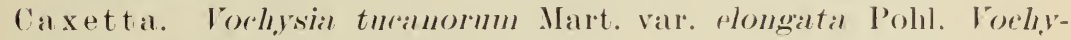
sialcele. Gummi und Bauhol\%.

(axicoem ville (Huxica.

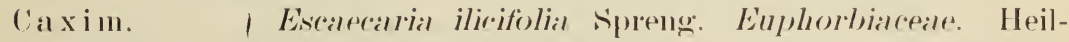
Caxilguba. i mittel.

Caxiri. Inclianerspeise von Mandiocea.

Caxixi. Schwarcher, schlechte Qualität Zurkelbranntwein.

Cay a ia vile T'apia do mato.

Cayarana. Cabloblea lineris ('. I)('. Meliareate. Bauhol\%.

C'ebià vide ('erneira.

Cebola branca. Weisse \%wiebel, vide cebola cerern.

Cebola brava. Wilde Zwiebel. Clusia Hilariana Sichlecht. Cintti-

ferae; Panrlatium guianensis Ker. Amarrllidacear. Heilnittel.

Cebola brava grande. Crosse wilde Zwiebel. Crimum scabrum.

Sims. Amiryllidaceare. Heilmittel.

Gebola cecem. Amaryllis belladommil Vellos. Amaryllidacear. Heilmittel.

Gebola do mato. Wallzwiebel. Viriffinial harldinthina Ker. Amaryllilaceare. Heilmittel.

C'ebolinlia do mato, virle Alho do mato.

('edro. C'edrelal Glassiorii. (C. I)C. Meliaceare. Gummi, Riurle und Hol\%.

Cedro batata. Knollen Ceder. I Cedrela fissilis Vellos. Meliareare. Cedro branco. Weisse Ceder. i Bauholz und Heilmittel.

Cedro rinzento. Graue Ceder. Oreodaphne splendens Meissn. Lanraceae. Bauholz.

Cedro rosa, vide Cedro.

Cedro serpa. Cedrela paraguariensis Roem. Mriaceat. (immui, Rinde officinell, Kistenholz.

Cedron. Lippia lycioides Stend. Verbenacease. Heilmittel. vide Paù amarello.

Cega machado. Axtstumpfer. Physocalymna scaberrimum Pohl. Physocalymna floridum Pohl. Lythraceae. Sehr hartes Bauholz. 
C'ega olhos. Blindmacher. Ascleptias chrassavical L. Asclepiadaceac. Heilmittel.

Celidonia. Frisis divaricata Spreng. var. auriculata DC. Compositae. Heilmittel.

Centaurea, vide Bocca de sapo.

Centaurea do inato. Wildes Trausendgüldenkraut. Dejanira nerrosa (ham. et Schlecht. Gentianaceae. Ersatz für Herb. centaurii.

C'entaurea minda. Kleine C. Schultesia angustifolia Grieseb. Gentianaceae. Bitteres Heilmittel.

Centaurea da terra. Einheimisches Tausendgiilden Kraut. C'entaurea melitensis L. Compositae.

Cepa cavallo. Xanthinm spinosum L. Compositap. Heilmittel.

Cereiba vide Mangue branco.

Cereiting a vide Mangue vermelho.

Cereja do mato. Waldkirsche. Trianosperma trilobata Cogn.

Cucurbitaceae. Heilmittel.

('ereja da purga. Purgiskirsche. Caraponiat perlata Cogn. Melothria

fluminensis Gardn. Cururbitaceate. Früchte drastisch.

Cerejeira. Kirschbaum. Mrrcianthes edulis Bg. Myrtaceas. Friichte essbar.

Cerejeira brava. Wilder Kirschbaum. Gilibertia monogyna E.

Mareh. Araliaceare. Frïichte.

Cerejeira da terra vide (ingeira brava.

Cerneira. Mimosa raesalpiniafolia Bth. Minosaceae. Holz.

Ceryba. Guillielma Mattogrossensis Barb. Rodrg. Palmae. Früchte.

Cevada. Gerste. Hordeum rulgare L. Gramineae.

Cevada santa. Heilige Gerste. Hordenm hexatichon D. Grannineare

Chá do Brasil. Brasilthee. Verbena erinoides Lam. Verbenaceate. Geträink.

Chá de bugre. Indianerthee. Rudgeal viburnoides Bth. Rubiaceae.

Chá cravo. Nelkenthee, vide C'raveiro ro mato.

C'há de frade. Mönchsthee. Lippia psendothea sch. Verbenaceat. Getränk und Heilmittel.

Chá inglez. Englischer Thee. Sirla rhombifolia L. Malvaceap. Emolliens.

C'há do mato. Waldthee. Buchnera rosea HBKth. Sicrophulariareare.

Chá sem medo. Furehtloser Thee. Lippia origanoides HBKth.

Verbenaceae. Heilmittel. 
Chá pedestre. Soldatenthee vide Chá de frade.

C'há rlos taboleiros. Lippia asperifolia Rich. Terbenaceate. Heilmittel.

C'há da terra. Tnrneral advocata Rich. Turnerareap und auch ricle Veronica da terra. Getriink und Heilmittel.

Chachim vide Mato olho.

Chagas. Troparolnm majus L. Troplueolareap Officinell.

Chagas mindas. Kleines Windkraut. ('hrmorarpns pentaphyllns

Don. Tropaeolaceae. Heilmittel.

Chagueira vide Barba de barata.

Cluam alot te vide Monjolo ferra.

Chamburì. C'ariral digitatil Aubl. P'apajalceare. Milch und Firucht.

Chanana. Turnera opiferal Mart. Tnrmerarrate. Heilmittel. Vide auch Nove horas.

Chapario bravo. Rhopalir romplicata HBKth. Protearoate. Holz.

Chapeo de couro. Lederhut. Erhinorlorus marrophrllus Mïll. Alismaceae. Heilmittel.

Chapeo de frade. Mönchshut, vide Bolsa de pastor.

Chapeo de sol. Schirmbaum. Anrelianal fisciculatil Sent. und Caavurana. Solanaceae. Heilmittel.

Chauá vide Massaranbuba branca.

Chiagari. Cnphea antisyphilitica HBKth. Lythrareate. Heilmittel.

Chibatan. Astroninm gravolens Jacq. var. hrasiliensis Engl. Anacarcliacea?. Heilmittel. Bauholz.

Chibui. Bauholz im Staate Amazonas.

Chi ca vide Carajúru.

Chich a vide Ararixá.

Chicha-rana. Falsche Chicha. Stermlia lasiantha Mart. Stermliaceae. Friichte essbar.

Chicla. Mandioccabier der Indianer.

Chigùa. Nectandra Turbagensis Nees. Lanraceae. Bauholz.

Chilca. Empatorimm dendrioisles Spreng. var. xyllophylloides Bak. Compositae. Heilmittel.

Chimbó vide Timbahyba.

Chique-chiqui. Crotalaria vitellina Kar. Papilionareap. Heilmittel.

Chiqui-xique. Opmntia brasiliensis Haw. Cartareate. Heilmittel.

Vide auch P'iassaba de Parì. 
Chirimoya. Anona cherimolea Müll. Anonaceae. Frucht essbar.

Chitotò vide Mahayjó.

Chocalha. Klapperpflanze. Crotalaria striata IC. Papilionaceae. Heilmittel.

Chonta. Guillielma insiguis Mart. Palmae. Frucht Nahrungsmittel.

Chopè. Gustavia longifolia Popp. Myrtaceae. Samen essbar.

Chorao. Amaranthus panicnlatus Mog. Amaranthaceae. Zierpflanze. Choyne vide Cuiéte.

Chrysiuma. Chusquea oligophylla Rupr. und Ch. capituliflora Trin. Gramineae. Rohr.

Chucalha. Aeschynomene racemosa Vog. Papilionaceae. Schlangenantidot.

Chuchú. Sechium edule Sw. Cucurhitaceae. Frucht Gemïse.

Chuchú bravo. Wilde C. Nierembergia hippomanica Meissn. Solanaceae. Heilmittel.

Chuco. Acanthorrhiza Chnco Dr. Palmate.

C'hupá vide Chopé.

Chupa ferro. Eisenverderber. Galipea jasminiflora Engl. Rutacea. Holz.

Churú. Couratari lineata Bg. Mrrtaceae. Banholz.

Chuvirangana. Tallesia chioccocroides Kth. Aporvnaceae. Heilmittel und Holz.

Cicantaa-ihua. Harz einer Protiumart, welches den Indianem zu Fackeln dient.

Cicatan. Protium divaricatum Engl. Burseraceae. Balsam und Harz als Elemi.

Cicuta. Schierling. Soliva anthemidifolia R. B. Compositale. Heilmittel, in den Nordstaaten hat Acarissoba diese Benennung.

Cidreira. Citronenbaum. Citrus merlica Risso Rutaceae.

Cidrilha. Citronenkraut. Lippia citriodora Kth. Terbenaceae. Heilmittel.

Ciguá vide Chiguá.

Cinco chagas. Fünfwundenblatt.) Sparattosperma vernicosum Bur. Cinco folhas. Fünfblatt. $\quad$ et K. Sch. Bignoniaceae. Enthält Alkaloid Sparattospermin.

Cinco folhas do campo vide Paú d'areo do campo.

Cindy-capetó. Tallesial punctata Spreng. Apocynaceae. Heilmittel. 
('inheirinlo to cam po. Cambessirlesia aspera DC. val. chamatrifolia C'ogn. Melastomaceae. Heilmittel.

('inll an omo. Melia azerlarach L. Melialceale. Heilmittel.

Cinzeira. Aschenbaum. Vochysia tucanormm Mart. var. microphylla Warm. Vochysiaceae.

Ciparabo vide Abutua.

Cipo d'Allo. Knoblauchbaum. Segnira floribunda Btlı. Phytolaccalceate; in Pernambuco Adenocalymna Sagotii Bur. et. K. Sch. Bignonialrears.

('ipo a mara gigante. Riesenbinderliane. Mnennal altisima DC. I'apilionaceare. Heilmittel und Bindemittel.

Cipo almessega. Elemiliane. Mikania biformis DC., M. hinsutissima I)C. Compositar. Officinell, sehr harzreich.

Cipo almiscar. Mikania ——? Wohlriechend. Compositar.

('ipo a marello. Gelbe Liane. Cuscntal tinctoria Mart. Cuscutacese. Heilmittel.

Cipo amargoso. Bittere Liane. Alunta radicans Rich. Menispermaceale. Simaba trichiloirles st. Hil. Simarubaceae. Heilmittel.

Cipo amara giqui. Fischreusenliane. Legiphila Mutisii HBKth. V.rlenareare.

l'ipo angico. I'iptarlenial mirranthil Bth. Mimosaceare. Heilmittel. Cipo d'a reo. Bogenliane. Galphimia brasiliensis Juss. Malpighiacex. Cipo a rimaru. Strychnos cogens Sclomb. Loganiaceate. Giftig. Cipo azul vide Viuva.

C'ipo ba una vide Bauna.

('ipo branco. Weisse Liane. Arlenoralymma hrarteatmm I'. DC. var. macrademum Bur, et K. Sch. Bignoniaceae Heilmittel und Flechtwerk.

Cipo branco de caboclo. Weisse Indianerliane. Adenocalymna macrophyllum P'. DC. Bignoniaceae. Flechtwerk.

Cipo branco de cerca. Zaunliane. Gouania discolor Bth. Rhamnaceae.

Cipo branco de rego. Coccoloba ilhensis Wedd. Polygonaceae. Heilmittel.

Cipo branco de S. Jo a vide Cipo branco.

Cipo caa opia. Marcgravia umbellata L. Marcgraviaceae. Heilmittel. 
('ipo caa razaca. Menora magnifica Bur. Bignoniaceae. Heilnittel und Flechtwerk.

('ipo caboclo. Indianerliane. Tetracera rolubilis L. Dilleniaceae. Heilmittel.

('ipo camaihúa. Panllinia thalictrifolia Juss. Sapindaceate. Heilmittel, Fischgift.

( i ipo canella de Jacù. Salacia Huminensis Peyr. Hippocrateaceae. Frucht.

Cipo de canôa. Idenocalrmma foreolatum Bur.: Memora consanguinea Bur. et K. Śch. Bignoniaceae. Flecht- und Bindematerial. C’ipo de carijó. Tetracerá oblongata DC.: Davilla rngosa Poir. Dilleniaceae. Heilmittel.

('ipo de carijó do campo. Inarlla elliptiral st. Hil. Hilleniaceate. Heilmittel.

Cipo carneira. Schafliane. Haemadictıon Gaudichaudii A. DC: Apocrnaceae. Heilmittel.

('ipo carneiro. Hammelliane. Inchieta exaltata Eich. Violacere. Heilmittel, vide auch Cipo de paina.

Cipo ca rurì. S'iphocampelus longepedunculatus Pohl. Lobelidceate. Heilmittel.

Cipo carurì-timbo. Odantadenial speciosal Bth. Apocinalceate. Giftige Milch.

Cipo catinga de paca. Elategurs trisperma Fr. All. Elatalgnaceat. Heilmittel.

Cipo catinga de porco vide Catinga de porco.

C'ipo de cerca vide Cipo branco de cerca.

Cipo de cesto. Korbliane. Arrabiraea coleoralyx Bur. et. K. Suh. Bignoniaceae. Flechtwerk.

Cipo de cesto grande. Hirtella riliata Mart. et Zuce. Rosaceae. ('ipo chato. Murnna altissinia DC. var. pilosula Bth. Papilionaceze.

Heilmittel.

Cipo cheiroso. Wohlriechende Liane. Arrabidaea subfastigiata Bur. Bignoniacear. Heilmittel.

Cipo de chumbo. Cuscuta racemosal Mart., C. umbellata HBKth. Cuscutareae. Heilmittel.

Cipo de cobra. Schlangenliane. C'issampelos Pareira L. Menispermaceae. Heilmittel und schlangenantidot. 
lipo roparcaballa vile Riacupari-cipo.

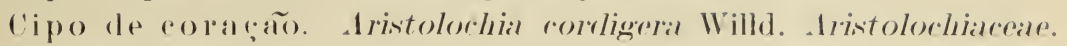

Heilmittel.

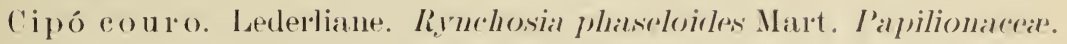

Binclemittel.

cipó rravo. Nelkenliane. Truanthes elegans Miers. Bignonialrade.

Heilmittel.

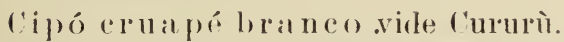

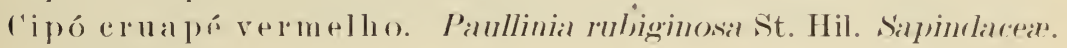

Fischlogit.

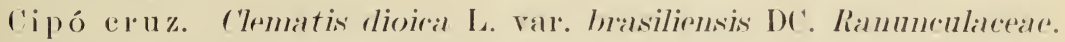

Heilmittel, virle auch ('aineal.

('ipo runaman. Euphorbial phosphorral Mart. Émphorbiareale.

He lmittel.

Cipo enruról. Serjand morlosal Radk. Silpindarpae. Gitig.

Anisolobus cururú Müll. Arg. Apocymareat. 'Toxisches

Heilnittel.

Cipo em vide Salsaparilha und Alaassus.

Cipo de escada. Bauhinial smilacina Steud. Mimosalrear. Industriell. C'ipo de gota. Vitis termatil Bak., I. pulcherrima Bak. Ampeli-

clacrate. Heilmittel.

Cipó guimgombó vicle Bucha campista.

Cipo guvra. I'leonotoma jasminifolinm Miers. Bignoniarease. Heilmittel.

Cijo-i. Davilla Incidil Presl. Dilleniareae. Heilmittel.

Cipo icica. Mikania glomerata Spreng. Compositae. Heilmittel.

Cipo imbé. Philoderlron imbé Schott. Arareare. Aeusserliches Heil111ittel.

Cipo imbiral. Canavalia pilrvifforal Bth. Papilionaceate. Binclenittel.

Cipo imbiri. Dioclea violacea Mart. Palpilionaceae. Heilmittel.

Cipo de inpigen. Flechtenliane. Stadtmannia depressa Fr. All.

Sapindaceae. Aeusserliches Heimittel.

Cipo issica vide Cipo icica.

Cipo Jaboty vide Fava de S. Ignacio.

Cipo de Jarrinha. Aristolochia brasiliensis Mart. Aristolochiacex.

schlangenantidot.

Cipo de lavandeira. Reifserkia smilacina Fr. All. Smilaceae. Heilmittel.

Cipo de leite. Milchliane, vide Teajú. 
C'ipo de macaco. Affenliane. Stenolobium velutinum Bth. Pappilionaceae. Heilmittel.

Cipu mainibú. Heilmittel in den Nordstaaten.

Cipo ma de sapo. Vitis Tweediana Bak. Ampelidacere. Heilmittel.

Cipo marmelinho. Adhatoda cydoniaefolia N. ab E. Acanthaceae. Heilmittel.

Cipo matador. Baumtödter. Lrostigma amazonicum Miq. Crticaceae.

Uipo molle. Weiche Liane. Pisonia aculeata L. Nyctaginaceae. Heilmittel.

C'ipo mororó vicle (ipo de escada.

Cipo mulatinha. Tetracera rotundifolia Smtlı. Dilleniaceae. Heilmittel.

Cipo mutà-mutì vide Cipo de escada.

Cipo orange. Memoral fulgens Bur. Bignoniale:ele. Heilmittel und Flechtarbeiten.

Cipo de paina. Wolliane. Araujia sericifera Brot. Asclepiadaceae. Heilmittel und Pffanzenwolle.

Cipo de pennas. Federliane. Stiperoma pelticera Müll. Arg. Apocrnaceae. Heilmittel.

Cipo quadrado. Serjania pancidentata DC. Sapindaceae. Fischgift. Ui po queimadeira. Brennliane. Dalechampsia Peckoltiana Müll. Arg. Euphorliaceae. Heilmittel.

Cipo quina. Chinaliane. Smilax syringoides Grieseb. Smilaceae. Heilmittel.

Cipo racha. Arrabidaea Blanchetii P. DC. Bignoniaceate. Bindemittel.

Cipo rego. Arrabidica Agnus castus I'. 1)C. Bignoniaceate. Heilmittel und Flechtwerk.

('ipo roxo. Doliocarpus Rolandri Gml. Dilleniacede. Heilmittel.

Cipo de s. João. Johannisliade (blüht am S. Johannestage.) Pierostegia vennsta Miers. Bignoniaceae. Heilmittel und zur Zierde in allen Gärten.

Cido de sapo. Froschliane, vide Cipo de paina und Rhinvinha do campo.

Cipo suma ride Piraguaia.

Cipo de tayuya ride Tayuya do Rio de Janeiro.

Cipo de timbo do campo. Serjania tristis Radlk. Sapindaceae. Giftig. 
Cipo de trindade. Dreieinigskeitliane, vide Cipo cravo.

Cipo tripa de galinha vide Tripa de galinha und Crotiga cipo. Cipo una. Clematis dioica L. Var. australis Eichl. Rannmrnlacpar. Bindemitiel und Heilpflanze.

Cipo una grande. Arrabirlaea plat.rphrylla var. acuminatil Bur. Bignoniaceae. Flechtwerk.

Cipo de vaqueiro. Viehhirtenliane. P'etastoma lencopogon Bur.

Bignoniareae. Heisst auch Cipo de fogo, als Ersatz der Stricke. Cipo vermelho. Rothe Liane. Melima tigarea Eichl. Dilleniareap. Heilmittel.

Claro d'ovos. Eiereiweiss. Cordial rufit-fisca Taub. Corliaceap.

Fruchtpulpe ähnlich wie rohes Hühnereiweiss, wirl genossen. Blätter Heilmittel.

Coagérécú. Xylopia fintescens Lubl. Anonaceap. Früchte Gewiirz. Coaginguva oder Coajinjuva und Coapoiba und Coaxinduba. Pharmarocycea anthelmintica Miq. Crticareas. Heilmittel.

Coahy. Getränk aus dem Fruchtfleische der Issahypalme.

Coari-íva. Tochysia grandis Mart. Vorhysiaceae. Heilmittel und Nutzholz.

Coaró bravo. Tagetes minnt:a L. Compositae. Heilmittel.

Coatindiva vide Corindiúva.

Cobió de Pará vicle Cubiós.

Coca vide Ipadú.

Coca-coca oder Coco-coca. Anlomyrcia Laroutteana Bg. Mrrtaceae. Frucht und Rinde.

Cocalleira vide Alcamphoreira.

Cacáo a marello und branco. Bauholz in Pernambuco.

Cochenilha vegetal. Vegetabilische Cochenille, vide Caticôa.

Coco de cachorro. Hundsnuss. Cocos Romanzoffiana Ch im. Palmae.

Coco de capim. Grassnuss. Cyperus esculentus L. Cyperareap. Speise.

Coco de purga: Purgirnuss, vide Anda-assí.

Coco de quaresma. Ostermnuss. Cocos picrophylla Barb. Rodr. Palmae. Frucht.

Coco de sapo. Froschnuss, heisst in Cearì die Baba de boi.

Coco da serra vide Acumão.

Coco de vassoura. Besennuss. Diplotheminm lencoralyx Dr. und I. campestre Mirt. Palmate. Bliithenrispe zu Besen. 
Coco de vinagre. Essignuss, vide Coqueiro vinagreiro.

Coentrilho. Pimpinellbaum. Zanthoxylum hiemale s. Hil. und

Z. culantrillo HBKth. Rntaceap. Starkriechende Blätter und Rinde Heilmittel.

Coentro da Colonia. Kolonie Coriander. Erynginm foptiolnm L.

Cmbelliferae. Heilmittel.

Coentro in Maranhã. Eryngium, juncenn Chum. var.juncifolinm

Urb. Lmbelliferas. Heilpflanze.

Coerana (von cui - Pfeffer, rana - fulscher). Cestrum Sendtneriannm Mart. Solanaceae. Heilmittel. In Alagoas ist Malouetia cestroides Mïll. Arg. Apocynaceae; in Minas Cestrum euanthes Schlecht.; in Rio de Janeiro Cestrum laevigatum schlecht.; in Rio Grande do Sul Cestrum Parqui L’Herit. Solanaceae.

Coérana brava. Wilde C. Solannm asperum Vahl. Solanaceae. Heilmittel.

Coerana do campo. Steppen C. Cestrum rorymbosum Schlecht.

Solanacear. Heilmittel.

Coerana de folha grande. Grossblätrige r. Cestrum sessiliflorum Schott. Solanaceae. Heilmittel.

Coerana pimenta. Pfeffer C., vide Coerana brava.

Coerana do Serra. Gebirgs C. Cestrum brarteatum Link et Otto.

Solanaceae. Heilmittel.

Coeté do mato (corrumpirt von caa - Blatt, eté-umfangreich).

Gonolobns macrocarpa Fr. All. Asclepiadareae. Giftig.

Coffó do diabo (cofo ist ein langer spitzer geflochtener Sack, wegen des langen Schlauches der Bliithe, und diabo - Teufel, in Folge des schlechten Geruches). Aristolorhia cymlifera Mart. var. abbreviata Duch. Aristolochiaceas. Wurzel Heilmittel und Schlangenantidot.

Cogo-logo vide Jequitiba branca.

Coité vide Cujeté.

Colher de vaqueira. Hirtenlöffel. Salvertia convallariorlora St. Hil. Vochysiaceae.

Colhos de gallo. Hahnhoden. Coltis arnleata Sw. Crticacear. Friichte essbar.

Comanda-giura. Vogelschote. Desmolium nncinatum DC. Papilionaceae. Heilmittel.

Comanda-hiba. Baumschote. Sophora tomentosa L. Papilionaceate. Heilmittel und Hol\%. 
C'omanda-merim mul Gomanduba-merim. Kleine Schote. Hirtella loractrata Mart. Rósalreale.

('om a rim vile ('mmarim.

Combetali vide Mamminha, de poreo.

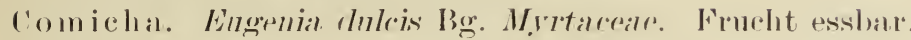

Comumbá bravo. Bamlolz in den Norrlstanten.

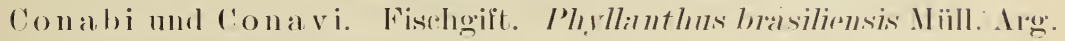
Enllorlialreale. Heilmittel.

Conambai merim vide Avenguinlas.

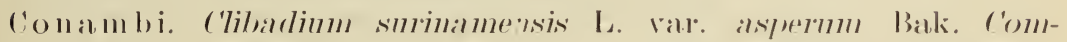
positare. Heilnitiol.

Condoé vide (iuariaro.

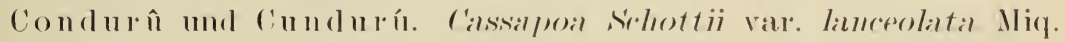
lloticalceale. Heilmittel. Holz.

Congonlad. Villanesial romgonhar Miers. Olarinalerar. In Rio de

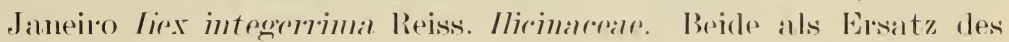
Mate.

Congonha brava. Wilder Mate. Rurlgral stemanth. Miill. Argeg. Rubiareaze. Blätter.

Congonha do campo. Steppenmate. Ilex Almotira Mart. Mlirinaceare. Bläitter.

Congonla de folla grande. Grosshliattriger Mate. Symploros varialiblis Mart. Śmblocalceare. Blïter.

Congonha de gentio. Indianer-Mate, vide Chà de bugre.

Congonha de Matto Grosso. Villaresial nunc. onata Rg. et Pr. Olicinaceale. Blätter.

Conta de cabra. Dorstenia bahliensis Klotsch. Drticareare. Heilmittel.

Contra cobra. Schlangenantidot. Aegiplitar salntaris HBKth. Verluenareale.

Contraherva. Gegengift. Dorstenia opifiral Mart. Urtirareae. Heilmittel und Schlangenantidot.

Copaia. Jacaranda copaia I). Don. Bignoniareare. Antisyphiliticum. Copaiba und Copaihiba. Copaivabalsamlieferanten. Caesalpineaceae. Im Staate Amazonas-Copaifera multijuga Hayn.

Im Staate Bahia - Copaifera coriacea Mart.

Im Staate Goyaz - Copaifera marginata Bth.

Im Staate Matto Grosso-Copaiferal elliptical Mart. und C. nitida. Mart. 
Im Staate Minas - Copaifera Langsdorffii Desf.

Im Staate Parà - Copaifera guyanensis Desf. und C. Martii Hayn.

Im Staate Paranà - Copaifera oblongifolia Mart.

Im Staate Piauhy - Copaifera confertiflora Btl.

Im Staate Rio de Janeiro-Copaifera officinalis Vell. und C. Langsdorffii Desf.

Im Staate S. Paulo - Copaifera trapezifolia Hayn.

Copaiba branca. Weisse C. Copaifera Langstorffii Desf var. grandifolia Bth. Caesalpineaceae. Weisses Holz.

Copaiba vermelha. Rothe C. Copaifera utilissima Fr. All. Ciresalpineaceae. Rothes Holz.

Copaí. Lucuma psammophila A. DC. Sapotaceae. Früchte und Bauholz.

Copa-ú va vide Copaiba.

Coqueiro. Cocospalms. Attalea princeps Mart. Palmae.

Coqueiro acumão vide Acumao.

Coqueiro acumao rasteiro. Kriechende Acumonpalme, vide Acumao rasteiro.

Coqueiro acuna vide Acuna.

Coqueiro acuri vide Acuri.

Coqueiro alicui, aliculi, alicuri, airi vide Aracuri.

Coqueiro amargoso. Bittere Palme, vide Paty amargoso.

Coqueiro an ajà vide Anajà.

Coqueiro anaja merim. Kleine Anajapalme, vide Anajà merim.

Coqueiro aricanga vide Aricanga.

Coqueiro aricar i vide Coqueiro uracari.

Coqueiro assahy-ai vide Assai-ai.

Coqueiro assahy merim vide Assai merim.

Coqueiro atitarà vide Rutin.

Coqueiro Ayri vide Brejauba.

Coqueiro azedo vide Butia.

Coqueiro baba de boi vide Baba de boi.

Coqueiro baba de boi grande vide Maria Rosa.

Coqueiro bacaba-i vide Bacaba-i.

Coqueiro bacaía a vide Bacaiúba.

Coqueiro de Bahia. Cocosnusspalme. Cocos nucifera Riss. Palmat.

Küstenkultur.

Coqueiro bocajá. Acrocomia Tatai Mart. Palmae. 
Coqueiro cabeçudo. Bauchige l'alme. Coros coronata Mart. Palmae.

Coqueiro cabeçudo do campo. Cocos leiospatha Barb. Rodr. I'almae.

Coqueiro caranì virle Muhi.

Coqueiro do Campo. Steppenpalme. Coeos campestris Mart.

Palmae. Wird auch Acumao benamnt.

Coqueiro Catalaa vide Bacaiuba.

Coqueiro de catarho. Śchleimpalme, vide Macaúba.

Coqueiro catolé. Cueos comosa Mart. I'almate.

Coqueiro catuté. C'oros botryophoral Mart. I'almale.

Coqueiro ehonta vide Turum-nassì.

Coqueiro corrozita vide Corrozita.

Coqueiro Datil vicle Tamareira da terra.

Coqueiro Dendé. Elapis guinensis L. Palmale. Kultivirt.

Coqueiro gangonzù vide Coqueira oauassù.

Coqueiro guriry und gury. Diplothemium campestre Mart.

Palmate.

Coqueiro imbury. Miplothemium candescens Mart. Palmae.

Coqueiro Indaya rasteiro. Atalea exigua Dr. Palmae.

Coqueiro Jaraiúva. Leopoldinia pulchra IVall. Palmae.

Coqueiro Jatitarà. Desmoncus riparius Spr. Palmate.

Coqueiro Jú. Astrocarymm lnumile Wall. Palmae.

Coqueiro Jubati. Schildkrötenpalme. Raphia vinifera P. de B.

var. taedigera Dr. Palmae.

Coqueiro Jupaty. Iriartea setigeral Mart. Palmae.

Coqueiro Jureva. Cocos acrocomioides Dr. Palmae.

Coqueiro macupi vile Curuâ-tinga.

Coqueiro maripa. Maximiliana maripa Mart. Palmae.

Coqueiro mocajà. Acrocomia sclerocarpa Mart. var. Wallaceana

Dr. Palmae.

Coqueiro munbuca. Astrorarym gynacanthum Mart. Palmae.

Coqueiro cauassú und úa ussú. Attalea speciosa Mart. Palmx.

Coqueiro piassaba vide Piassaba.

Coqueira da praia. Kïstenpalme. Diplothemium maritimum Mart.

Palmae.

Coqueiro paxiúba vide Coqueiro Jupaty.

Coqueira paxiúba majerona. Martinezia caryotifolia HBKth. Palmae. 
roqueiro de quaresma. Cocos flexuosa Mart. Palmae.

Coqueiro de sombra. Dachpalme. Copernicia tectornm Mart. P'alluale.

Coqueiro tarampabo. Oenocarpus tarapanbo Mart. Palmae.

Coqueiro tiassé vide Coqueiro yayúa.

Coqueiro tiú. Attilea romptia Mart. Palmate.

Coqueiro ubim. Geonoma paniculigera Mart. Palmae.

Coqueiro ubim assù. Calyptronoma robusta Trl. Palmae.

Coqueiro ubim merim. Geonoma acaulis Mart. Palmae.

Coqueiro ubussù. Manicaria saccifera Gaertn. Palmae.

Coqueiro uricari vide Aracuri.

Coqueiro uricari-iba. Attalea excelsa Mart. Palmae.

Coqueiro uvarii vide Coqueiro ubussù.

Coqueiro vinagreiro. Essigpalme. Bartris maior luce. Palmax.

Coqueiro Yayúa. Attalea Hmmbolltiana.

Coqueilo Yatai. Cocos Yatay Mart. Palmate.

Coquilho. Aldina discolor Spruc. Caesalpiniaceate.

Coquinho. Phyllanthus distichns Müll. Arg. Enplorlbiaceate. Heilmittel.

Coquinho babà. Desmoncus setosns Mart. Palmae.

Coração de boi. Ochsenherz, vide Miloló.

Coração de Jesus. Mikania officinalis Mart. Compositap. Heilmittel.

Corac;a de negro. Negerherz. Lonchorarpms serirens HBKth. Papilionaceate. Heilmittel. Albizzia Lebbek Bth. Minosiareale. Splint schwarz. Samen Heilmittel.

Coraçao da rainha. Königinherz, vide Cherimolia.

Coração verde. Grünharz, vide Bibirù.

Coral. Jatropha multifirla L. Euphorliareae. Wundbalsam.

Coral azul. Blauer Coral. Faramea Nettoana Mïll. Arg. Rnbiacear. Heilmittel.

Corda vegetal. Vegetabilischer Strick. Rhipsalis Lindluergiana K. Seh. Cartalcale.

Corda verde. Griner Strick. Rhipsalis grandiflora Haw. Cactacep. Cordão de frade. Mönchsgiurtelstrick. Leonitis nepetapfolia R. Br. Labiatae. Asthmamittel.

Coldaro de S. Francisco. Lencas martinicensis R. Br. Aromaticum. Coré bravo. Ipomopa fistigiata Sw. var. vulgaris Meissn. Convolvulaceare. Heilmittel. 
Corimbó. (Corrumpirt von curuba-wohlriechend, imbe-Sohling-

pflanze.) ('romastus pulcher Bur. Bignoniaceap. Heilmittel.

Corindiba. Celtis hrastiensis Garel. Drtirareale. Fiebermittel.

Corizó. Rohr. Gualna exalata Doell. Giraminrale.

Corneiba vide Aroeira vermelha.

Corôa vide Cróa.

Corôa de frade. Mïnchstonsur. Erhinorartus rontreteins Lehm.

Cartarrate.

Corôa de frade do Sertao. Erhinocartus plarentiformis K. Seh.

rilctilceale.

Coronha. Flintenkolben. Diorlea rufescens Bth. Papilionareate.

Corobó. Chloris testichophyllir Lagasc. Cramincale.

Corona-chris vide Espongeira.

Corongo. Complnena lenrorephala Mart. Amarantarear. Heilmittel.

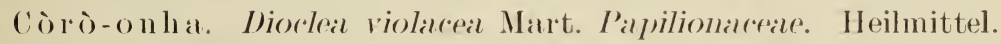

Corrente. Telanthora firoirlea Moq. Amarantareate. Heilmittel.

Corrozita. Cocos Orinocensis spreng. Palmae.

Cortica. Korkbaum. Piptarlomial falrata Benth. Mimosacear. Apeiloa

asperä Aubl. Tiliarrase. Korkersatz.

Cortiça raiz. Wurzelkork, vicle Tabebuja.

Corticeira. Erythrina rista galli L. Papilionareae. Heilmittel.

Cossa-cossa brava. Wilder Kratzer. Solamm apiculatum Sendt.

Solanacare. Blitter.

Cossa-cossa mansa. Milder Kratz r. Solanmm lanceaefolium Jace.

var. volubile Sendt. Solanilreate.

Cotó-Cotó vide Chà de bugre.

Cotó mirim. Ruclgea Frankavillama Müll. Arg. Ruliareae.

Couma rú vide C'úmarú.

Coumarú-rana. Falsche Tonkabolnne. Dipteryx oppositifolia Willd.

Palpilionilceale. Simen.

Couve de mato. Waldkolıl. Poropliyllum ruderale Cass. Compositae. Heilmittel.

Couve do mato Virgem. Trwaldkohl. Psyrhotria colorata Miill.

Arọ. Ruliaceare. Heilnittel.

Couvinha craveiro. Nelkenkohl, vide Craveira de urúbú.

Coxi-coem vide Guaxica.

Craveiro do campo. Steppennelke. Falyptranthes variabilis Bg.

Myrtareate in Rio (irande. Isostigmal pencerlanifolinn Less. Compositiae. 
Craveiro de Ma ra n hao. Nelkenbaum. Decypellium raryophyllatum

Nees. Lanraceap. Aromatische Rinde officinell.

Craveiro da terra. Gemeiner Nelkenbaum. Calyptranthes aromaticus st. Hil. Myrtaceae. Die aromatischen Früchte officinell. Craviua de lagarto. Eidechsen Federnelke. Jussiaea linifolia Vahl.

Onagrareas. Heilmittel.

Cravo bravo vide Coaró bravo.

Cravo de defunto. Todtennelke. Tagetes erecta L. und T. patula

L. Compositae. Heilinittel.

Cravo sylvestre. Wille Nelke vide Coaró bravo.

Cravo de urubú. Aasgeiernelke. Porophyllum lineare DC. Compositire. Heilmittel.

Cravorana. Falsche Nelke. Ambrosia artimisiaefolia L. Compositæ.

Heilmittel.

Crindiúva. Sponia mirerantha DC. Hrticaceap. Heilmittel.

Crista de gallo. Hahnenkamm. Celosia ristata Moq. Amar.ın-

thacpae, vide auch Aguarà-cuinha assì.

Crista degallo da terra. Celosia brasiliensis Moq. Amaranthacex. Crista de mor;a. Clitoria glyrinoirles DC. Papilionaceae. Heilmittel und zierpflanze.

Crista de negra. Clitoria glveinoirles DC. var. aurantiaca Bth. Papilionaceae. Zierpflanze.

Criúva. C'lusia Crinva St. Hil. Guttiferae. Harz und Rinde.

Crixiúm a vide Chrysiuma.

Cróa, Crúa a, Curúra, Curúlıa. Sicana odorifera Naud. Cucurbi-

taceas. Der sehr wohlriechende Kïrbis benutzt.

Crúapé vide Cururú.

Cruzeiro. Kreuzbaum. Meclienxia cordigera Mart. et Zuer. var. gennina Müll. Arg. Rubiaceare. Heilmittel.

Cuambá, Cuambí. Biclens graveolens Mart. Compositae. Heilmittel.

Cuaró, Quaro. Throgallis brachrstachys Lindl. Malpighiaceae. Heilmittel.

Cuarú-batinga, Curúbatinga. Centrolobium robustum var. macrochaete. Papilionacene. Vorzïgliches Bauholz.

Cubios. Solanmm sessiliflornm Don. Solanaceae. Heilmittel.

Cubyo. Lucuma laterifolia Benth. Sapotaceae. Früchte.

Cucurú. Anisolobus cncuru var. grandifolins Mïll. Arg. Apocynaceæ. Giftig. 
Cuguassú-remiú vide Aypim.

Cuhura-quam vide Paì Brasil.

Cuiarana, Cuirana. Secondatia arborea Müll. Arg. Apocynaceae. Heilmittel.

Cuiéte, Cujeté. Kalebassenbaum. Crescencia cujete L. Bignoniaceas. Zu Gefässen. Kultivirt.

Cuíte do mato. Anclira humilis Mart. Papilionareare. Friehte Heilmittel.

Cui-hem-jurimú. Capsicum annum L. var. grossum Steud. Solanaceale. Gewïrz.

Cu i-hem-ozú. Capsicum annum L. var. corrliforme Steud. Solanarear. Gewürz.

C'ui-hem-peia. Cui-hem-cuiepia. Capsicum cerasiforme Vellos. Solanaceae. Gewïrz.

Cui-hem-saban. Capsicim ovatum Vellos. Solanarean. Gewürz. Cuipana, cuipuna vide Caipuna.

Cu i-peúna. Tibouchina mutabilis Cogn. Melastomaceae. Heilmittel. Cuitello. Camptosema rubicundum Hook. et Arn. Palpilionaccale. Cujamarioba vide Fedegosa.

Cujumary. Aydendron cujumary Nees; in •den Nordstaaten Oreodaphne guyanensis Meissn. Lanraceae. Heilmittel und Nutzholz.

Cuma-á vide Sorva.

Cumamery vide Sorveira.

Cum anda-taia. Dolichos Lablab L. Papilionaceae. Nahrungsmittel. Cum and a-uassì. Grosse Schote. Nissolial fruticosa Jacq. Papilionaceae. Bauholz.

Cumari. Torresi Cearensis Fr. All. Caesalpiniaceate. Bauholz und Heilmittel.

Cumarú. Dipterix odorata Willd. Papilionaceale. Lieferant des Tabac tonco.

Cumati. Allemanda Aubletii Pohl. Apocynaceae. Wurzel u. Blätter. Cumaty. Psidium albidum Camb. Myrtaceae. Früchte.

Cumazah i. . Aspidosperma Gomezianum A. DC. Apocynaceae. Rinde, Bauholz.

Cumbai-merim vide Sepipira.

Cumbarú. Dipterix tetraphylla Spruc. Papilionaceae. Tabac tonco beigemischt.

Cumbarú-rana. Falsche Tonkabohne. Dipterix oppositifolia W. Papilionaceae. 
Cumbeba. C'erens triangularis Haw. Cactaceae. Heilmittel.

C'umbery vide Bartì.

Cumbira. Mimosops subserirea Mart. Sapotacear. Milch, Rinde, Frïichte, Holz.

Cum deira vide Paù situto.

Cumichá und Cumúchá. Erythroxylum passarinum Mart. Errthroxylaceate. Holz und Rinde.

Cunabi. Ichthyothere cumabi Mart. C'ompositare Fischgift.

Cunami vide Conambi.

Cunca. Knollige saftreiche Answiichse an den horizontalen Wurzeln

des Imbuzeirabanmes, dienen in der trockenen Zeit dem Vieh zur Lïschung des Durstes.

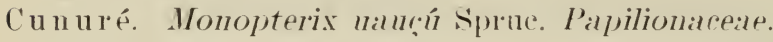

Cunuri. Enphorbia genirulatil. Enphorliareate. Heilmittel.

Cupauba-rana. Falscher Copairal. Eperua purpurea Bth. Casesalpiniaceate. Bauholz.

C'upay und C'upuiba vide Copaiba.

Cuphea. Cuphea aperta Kophn. Lrthrareale. Ofticinelles Heilmittel. Cupiero vide Ćaruró hranco.

Cupiuba. Zanthoxylum nigrum Mart. Rutaceate. Bauholz. Blätter. Cupiuba branca. Weisse (". Mrrcial errthroxylon Bg. Mertaceau. Bauholz.

Cupú-assì. Meltonia Intea Fr. All. Malvaceate. Heilmittel.

Curabi. Die kleinen Giftpfeile der Indianer.

C'urali vide Ehano.

Curalleira. Croton antisylhiliticnm var. gemninus Müll. Arg. Enphorbiaceae. Wurzel Heilmittel.

Curamari. Arrabidaea inarqualis Baill. Bignonialceate. Heilmittel. Curaré. Pfeilgift, vide Urari.

Curata. Cocroloba nitida HBKth. var. cortata Meissn. Polrgonaceae. Heilmittel.

Curáu. Guaranibenennung für Canzica.

Curau a vide Caróa.

Curititiba und Curatatina ride Cutitiriba.

Curi-y und Curi-úva. Tupibenennung für Arancarlal brasilianal

Richt., vide Pinheiro.

C'urumbà vide Sambongo.

Curuâ und Curuâ-juquira. Salz C. Attalea spectabilis Mart.

Palinae. 
Curuâ-piranga. Rothe ('. Attalea spertabilis Mart. var. typlial Dr. Pallilate.

('uruâ-pixuиa. Schwarze (\%. Attaleal spertabilis Mart. var. polyandral Dr. Palmile.

Curuâ-tinga. Weisse (\%. Attilea spectabilis Mart. var. monospermal Barb. Rodr. Palmae.

('uruâ-y. Wasser ('. Attalea mirrorarplal Mart. Palmile.

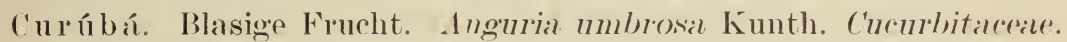

Heilmittel.

Curuba i-m irim vile Sepipira.

Curubatinga vicle Cuarí-batinga.

Curuè̀ und ('urueui vide Taeamalaacal.

Curui-caa. Brickellial diflusial A. Grey. Compositae. Heilmittel.

Curuiri. Phrlloralyx Luschmathianus Bg. Mytareate. Heilmittel und Friichte.

Cururé. Apinagia fincoirles Tul. Porlosteminceare.

Cururi vide (Ma-rerì.

Curupica-iba vide Cajazeiro do mato.

Cururì̀. Froschkraut, vide Cipo cururù.

Curvelha vide Bolsa de pastor.

Cutia. Esenbeckia leiorarya Engl. Rutaceate. Bauholz.

Cutiará. Bauholz in Parà.

Cuticaem. Arlenostephamus Sellowii Kl. Proteaceae. Heilmittel.

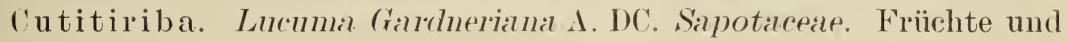
Nutzholz.

Cutucanhé. Rhopala insignis Fr. Alle. Protenceae. Nutzholz.

Cuturubá vide Cutitiriba; corrumpirt von Oiti-turuba.

Cuspe de tropeira vide Espinho de cachorro.

Dachoché vide Milho d'ag'ua.

Da in a de lagos. Seedame, vide Igrupé.

Damina. Thrnera riffisa Willd. Tnrmeraceate. Heilmittel.

Datil vide Tamareira da terra.

Dedal. Fingerhut. Lafoensia densiffora Pohl. var. anculata Koch. Lythraceae. Heilmittel.

Dendé vide Coqueiro Dendé.

Dente de Leão. Lörvenzahn. Smilax phylloloba Mart. S'milaceae. Allemanda Doniana Müll. Arg. Apocynaceae. Heilmittel.

Dendé da terra. Einheimische Elä̈s melanocoeco Gaertn. Palmae. 
Diconroque. Indianerbohne. Saguinea Peckolti sch. Urticacteat.

Früchte. Nahrungsmittel der Indianer Coroados.

Dicuri vide Aracuri.

Didy de porteira. Commelinal Pohliand sueub. Commelinaceate. Heilmittel.

Doido. Strauch in Ceará, wenn rom Vieh gefressen wirkt tödtlich. Dormideira vide Feiao de arvore.

Douradinh a. Goldkraut. Psychotria xanthophylla Müll. Arg. Ruliaceae. Waltheria Douradinha St. Hil. Büttneriaceae. Heilmittel. Douradinha do campo. Steppen-Goldkraut. Psychotria rigida var. aurata Müll. Arg. Rubiaceae. Heilmittel.

Doura dinha falsa. Falsches Goldkraut. Brrsonima verbascifolia Richt. Malpighiaceae. Heilmittel und '/ierpflanze.

Don Bernardo. Psychotria tetraphylla Müll. Arg. Rubiaceae. Heilmittel.

Dragao fedorenta. Stinkende Drachenpflanze. Monstera pertusa De Vries. var. lanata Schott. Araceae. Wurzel officinell.

Ebana. Brasil. Ebenholz. Tecoma leucoxylon Mart. var. salpingophora K. Sch. Bignoniaceae. Nutzholz.

Efó. Speise von Carurú, Krabbe und Capsicum, etc.

Egrió. Kresse. Nasturtium pumilum Camb. Cruciferae.

Eguarà. Gyneriuni saccharoides HBKth. Gramineae. Futterpflanze. Elemi vide Alnuessegueira.

Embauvarana. Bauholz.

Embeaxiò vide Membú-iaxió.

Em beguaca. Heilpflanze in Alagoas.

Embigo do porco. Schweinsnabel. Xanthoxylum rinereum Engl. Rutaceae. Bauholz.

Embigo da freira. Nonnennabel. '/uckerplätzchen mit Nandioccamehl.

Embira. Bastbaum. Funiferal fusciculatit Meissur. Thymelialceat. Faserstoli.

Embira branca. Weisser Bastbaum. Funiferal utilis Leand. Thymeliaceae. Apeiba cymbalania Arruda Tiliaceae. Daphnopsis lirasiliensis Mart.; Daphnopsis Martii Meissn. Thymeliaceae. Faserstoff. vide auch Pindahyba.

Embira de caboclo. Indianerbast. Schoenobiblus daphnoides Mart. Thymeliaceae. 
Embira de folha lisa. (ilattblittriger Bastbaum. Bombax gracilipes sch. Bombaceale.

Embira guass ì und Embir-assì vide Imbir-assì.

Embira do mato. Waldbasthaum. Helirteres ovata Lam. Sterculeaceate. Faserstoff.

Embira da serra. Gebirgsbastbaum. Gnatteria apodocarpa Mart. Anonaceare.

Embiriba preta. Schwarzer E. (Conratari macromarpa Mart. MYrTEmbiriba vermelha. Rother E. f tareate. Banholz und Feserstoff. Embirofanha vide Barriguda.

Eubiń a marello. (ielber F. Inaguetia Pohliana Mart. Anonareae. Möbelhol\%.

Eumbiú branca. Weisser E. Dagnetial brarteosal Mart. Inonaceale. Bauholz.

Emburá-be mbó. Ipomoea fistigiata Sw. var. platanifolia Grieseb. Convolvulaceare. Heilmittel.

Emburana mansa vicle Acajabeira.

Embury vide Imburi.

Emby ì. Guatteria alba Sald. Anonaceas. Möbelholz.

Endacia. Baum. Heilmittel.

Endro. Dille. Anethum graveolens L. Umbelliferae.

Enforcadinha. Orchidareare. Heilmittel.

Engà vide Inga.

Engasca vacea. Kuhsticker. Lucumal montona Fr. All. Sapotacex.

Früchte werden vom Vieh genossen und verstopfen die Speiseröhre. Enquiri. Chrysophyllnm tomentosum Fr. All. Sapotaceae. Bauholz. Rinde Heilmittel.

Enxerto de passarinho. Vögelsetzling. Strutanthus Salimanni

Eichl.; Psittacanthus biternatus Blum. Loranthacear. Heilmittel. Ervaca. Heilpflanze in Alagoas.

Ervilha. Erbse. Pisum sativum L. Papilionaceae.

Ervilha de Angola. Angolaerbse, vide Guando.

Escadinha. Erythroxylum ochranthum Mart. Erythroxylaceae.

Heilmittel und Nutzholz.

Escobá. Bürstenkraut. Heterothalamus spartioides Hock et Arn.

Compositae. Heilmittel.

Esconde fogo. Melastomaceae. Nutzholz.

Esfola bainha. Xylopia ochrantha Mart. Anonaceae. Heilmittel. Esparto da terra. Brasil. Spartogras. Pharus glaber HBKth.

Gramineae. Viehfutter. 
Espelina. Perionthopodus espelina Manso. C'ururbitaceae. Wurzel Drasticum und Epilepsiemittel.

Espelina falsa. Falsche E. C'litoria guianensis Benth. Papilionacoare. Heilmittel.

Esperta. Tabernaemontana lapta Mart. Lpocrnaceae. Rinde und Blätter Heilmittel, enthalten Alkaloid.

Espiga de sangue. Blutkolben. Helosis brasiliensis Sichott. Balanaphoraceate. Heilmittel.

Espiga secca. Trockner Kolben. Lathrophrtum Perkolti Eichl. Belanaphoraceae.

Espig̨ão. Pedilanthus padifolinm Poit. Euphorliacean. Heilmittel: enthält Alkaloid.

Espigão bravo. Perlilinthus retusus Benth. Euphorliareap. Beide wirken toxisch.

Espinha de agulha. Nadeldorn. C'huquiragual vagans Bak.; C'huquiragua tomentosa Bak. Compositae. Heilmittel.

Espinha branca. Weissdorn. Machaoniat spinosa ('ham. et schlecht. Heilmittel.

Espinha de cachorro. Hundsdorn. Soliva sessilis R. et P. Compositar. Heilmittel.

Espinha de carneiro. Schafsdorn, vide Cepa cavallo.

Espinha do diabo. Teufelsdorn. Lesmoncus horridus spl. et Mart. Palmap. Flechtarbeiten, und Wurzel Heilmittel.

Espinha de Judeo. Judendorn. Mrlosma Salzmanini ('h. Bixareap. Heilmitteì.

Espinha de Maricá. Mimosa sppiaria Bth. Mimosarease. Blätter Asthmamittel.

Espinha miuda. Kleindorn. C'aliiandrar Peckolti Bth. Mimosaceap. Gummi und Blätter.

Espinha roxa. Violetrother I)orm. Piptarlenia polvptera Bth. Mimosaceate. Rinde.

Espinha de rintem vide Mamminha de porco.

Espinha vermelha. Rothdorn. Barnarlesia rosea Lindl. Compositae. Heilmittel.

Espinheiro am arello. Gelber Dornbaum. Mimosil sulsericea Bth. Mimosiareate. Nutzholz.

Espinheiro ameixa. Pflaumendornbaum, vide Ameixeira da terra. Espinheiro ameixa brava. Wilder Pflaumendornbaum, vide Ameixeira de espinho. 
Espinheiro das bordas da estrada. Wegedornbaum. Mimosa pudica L. forma hispirlior Bth. Minosareap. Heilmittel.

Espinheira da cerca. Kaundornbamur. Mimosa olongarla Benth

Minosaceate. ('artenumziiumung.

Espinheiro corne de bode. '/iegenhorndornbaum. I Minosia miriEspinheiro corne de veado. Rehlorndornbaum. I adina Benth.

Mimosiareate. Rinde Heilmittel.

Espinilho. Kleiner Dornbaum. Glerditsolitia amorphoirles 'Taub. Caesalpineareale.

Espiradeira vide Alandro.

Esponja de rede. Netzschwamm. Initophorar rampanulatal Nees. Finngi. Giftpil\%.

Esponja da terra. Erdschwanm. Sylalinm fingosmm schott. Balanophoraceae. Heilmittel.

Espongeira. Acacia Farmesiana Willd. Minosalcate. Blïthen und Samen.

Espora de gallo. Hahnensporn, vide (irão de gallo.

Estaca cavallos. Pferdeberuhiger. Gratiola pernviana L. Śrophularaceate. Heilmittel fïr 'Thiere.

Estalador. Rutireae. Bauhol\%.

Estanca sangue. Blutstiller. Piptorarplir leprosa Bak. ('ompositae. Heilmittel.

Estoraque. Storaxbaum. Strrax mervornm I. DC. Styraceae. Heimittel und Holz.

Estramonia. Stechapfel. Datnral Stramonium L. Solanaceae.

Estrella do Norte. Nordstern. Ramrlia latifolia Lam. Rubiaceap. Zierpflanze.

Estremosa. Lagerstroemia indica L. Lytliraceae. Gartenpflanze. Evarate-seń. Heilpflanze in Alagoas.

Fa cheira. Fackelpflanze. Epiphyllum acnminatum K. Sch. Cactacex. Fachina. Reisholz. Cassupa verricosa HBKth. Rubiaceae. Heilmittel.

Faia. Buche. Cordia sylvestris Tres. Cordiaceae. Nutzholz.

Farinha secca. Trockenes Mehl, vide Paú rei und Mangue de mato. Fava. Bohne. Vicia sativa L. Papilionaceae. Nahrungsmittel.

Fava bico de papagaio. Papageischnabelbohne. Phaseolus panduratus Mart. Papilionaceae. Nahrungsmittel.

Fava branca. Weisse Bohne. Vicia faba L. Papilionacene. Nah rungsmittel. 
Fava branca miuda. Kleine weisse Bohne. Phaseolus nana L. Papilionareae. Nahrung.

Fava do café vide Pos de mico.

Fava cheirosa. Wohlriechende Bohne, vide C'umarù.

Fava cobra. Schlangenbohne. ) Canavalia gladiata DC. Papilio-

Fava contra. Antidotbohne. $\}$ naceas. Same. Antidot bei Schlangenbiss.

Fava figado de galinha. Huhnleberbohne. Papilionaceae. Heilmittel in Alagoas.

Fava de impigem. Flechtenbohne. Crudya obliqua Grieseb. Caesalpineaceae. Same. Heilmittel.

Fava juca. Heilmittel in Cearà.

Fava do mato. Wilde Bohne. Pitherolobinm multiflorum Benth. Mimosaceae. Heilmittel.

Fava monjoló. Stampfbohne. Psophocarpus longepedunculatus Hask. Papilionareae. Heilmittel und Nutzholz.

Fava olho de peixe. Fischaugenbohne. Papilionaceae. Heilmittel in Alagoas.

Fava de quebranto. Hexenbohne, vide Fava contra.

Fava rama. Zweigbohne. Canavalia bonariensis Lindl. Papilionarear. Heilmittel.

Fava de rap̀̀. Schnupftabakbohne, vide Cumarì.

Fava rim de paca. Pakanierenbohne. Papilionaceae. Volksmittel in Alagoas.

Fava riscada. Gestreifte Bohne, vide Feijão miudo.

Fava sangue de boi. Ochsenblutbohne, vide Feijão roxo. Varietät. Fava Santo Ignacio. St. Ignazbohne. Sicydium monospermum Cogn. Fenillea delticlea Cogn. et $F$. trilobata L. Cucurbitaceae. Samen Heilmittel.

Fava de sete seinanas. Bohne in sieben Wochen reif. Varietait von Phaseolus nana L. Nahrungsmittel.

Fava tonco vide Cumarù.

Faveira. Bohnenbaum. Bauholz. Mimosaceae.

Faveira brava. ! Bauholz in Matto Grosso.

Favinha. Kleine Bohne. Cleobnlia diocleoides Kth. Papilionaceae. Heilmittel.

Fedegoso. Cassia ocridentalis L. Caesalpineaceae. Samen und IVurzel. 
Fedegoso grande. Cassia quinqueangulata Rich. Caesalpineacear. Wurzel.

Fedegoso legitimo. Aechte F. Cissial affinis Bth. Cinesalpineàcear. Wurzel officinell.

Fedegoso do mato. Wilde F'. C'assial pubescens Iacq. C'aesalpineaceae. Rincie und Wurzel Heilmittel, vide auch Mata pasto.

Fedegoso do mato virgem. Urwald F. C'assial speciosia Schrat

Caesalpineaceae. Cassial bijugal Vog. Rinde otficinell, enthïlt ('hrysophansäure.

Fedegoso do Parà vide Aguará-cninha-assí und Mata pasto. Fedegoso de Pernambuco. Heliophrtum elongatum D(\% Heliotropiear. Heilmittel.

Federal. Oyclaea Bonplandiana Kth. C'ompositae. Volksmittel. Fedorento. Stinker, vide ('ipo cı'1\%.

Feijão anã: Kwergbohne, vide Fava branca miucla.

Feijao de arvore. Baumschminkbohne. Sesbania l'aulensis Barb.

Rodr. Papilionaceate. Volksheilmittel.

Feijao de boi. Ochsenschminkbohne, vide Comandahiba.

Feijao de boi da capoeira. Fapparidaceap. Samen sollen giftig sein.

Feijao branco. Weisse Bohne. Phaspolns vulgaris L. Papilionacear. Nahrungsmittel.

Feijao branco da sopa. Weisse Suppenbohne. Varietait der Vorhergehenden.

Feijao bravo. Wilde Bohne. C'anavalia obtusifolia DC. Papilionacrae. Samen Heilmittel.

Feijao de caboclo. Indianerbohne, vide Diconroque.

Feijao de capoeira. Gebüschbohne. Crotolaria stipularial Desv. Papilionaceate. Samen Volksheilmittel.

Feijao caracol. Schneckenbohne. Phaseolus caracalla L. Papilionaceae. Nahrungsmittel.

Heijao carapato. Holzbockbohne. Holichos sphiserospermuns I)(. Papilionacere. Nahrungsmittel.

Feijao de castanha. Kastanienbohne, vide Fava branca miuda. Feijao côco. Cocosnussbohne, vide Barù.

Feijan fava bravo. Wilde Schotenbohne. Canavilia versicolos Barb. Rodr. Papilionaceae. Samen Volksmittel.

Feijao fradinho. Pfäffleinbohne. Dolichos monachalis Brol. Papilionaceate. Nahrung. 
Feịao gitirana. Laternenträgerbohne. Zornia diphrylla Per's var.

leptophylla Bth. Papilionaceae. Vielifutter.

Feijao grande. Grosse Bohnenschote. Adenocalvnnna miarginatum

P. DC. Bignoniaceae. Volksheilmittel.

Feijao da India vide Cumanda taia.

Feijao do mato. Waldbohne. Cassia exsudans Bth. Caesalpineacea. Volksmittel.

Feijao merim. Kleine Bohne. Clitoria ternata L. Papilionaceate. Volksmittel.

Feijao miuda. Winzige Bohne. Phaseolus inanoenus L. Papilionacere. Essbar.

Feijao da praia. Strandbohne, vide f'omanda hiba.

Feijao preto. Schwarze Bohne. Phaseolus derasus Schrank. Papilionaceae. Allgemeines tägliches Nahrungsmittel der Brasilianer.

Feijao roxa. Violetrothe Bolnne. Phaseolus lunatus L. var. macrocarpus Bth. Papilionareae. Nahrungsmittel.

Feijao de sete semanas. Siebenmonatshohne. Centrosema platycarpum Bth. Papilionareae. Essbar.

Feijao timbó. (Giftbohne. Phaseolus semierectus L. Papilionarcae. Roher Same soll giftig sein.

Fel da terra. Erdgalle. Lophophytum mirabile Mart. Balanophoraceae. Tejanira erubesiens Cham. et sichl. Gentianaceae. Heilmittel, vide auch C'afferana.

Feto macho. Farnkrautwurzel (männliche). Polypodium incanum

L. var. squalidum B. Polypodinm percussum Cav. Polypodinm crassifolinm L. Polypodium lepiclopteris Kunze. Aspleninm serratum L. Polypodiaceae. Wurzel Bandwurmmittel.

Fiandeira. Guttiferae. Heilmittel in Alagoas.

Figado de galinlı. Huhnsleber. Bauholz.

Figueira. Feigenbaum. Ficus carica L. Lrticaceae.

Figueira brara. Wilder Feigenbaum. Pharmacostrea rermifingal

Miq. Crticacere. Milch anthelininticum. Vicie Pharm. Rundschau. Figueira do inferno. Feigenbaum der Hölle, zufolge der furchtbaren Stacheln. Opuntia brasiliensis Haw. Cactaceae. Vide auch Estramonia.

Figueira do mato. Waldfeigenbaum. Trostignna eximiunn Miq.

Lrticaceae. Milch officinell. Vide Ph. Rundschau.

Figueira terrestre. Erdfeige. Dorstenia bryoniaefolia Mart.

Dorstenia brasiliensis Lam. Dorstenia opifera Mart. Crticaceae. Wurzeln officinell. Vide Ph. Rundschau. 
Flecha. Pfeil, vide Uba.

Flecha do diabo. Teufelspfeil Bartric acanthocarpa Mirt. var. crispata Dr. Palmae.

Flor de abril. Aprilblume. Dillenia speciosa Thun. Dilleniaceae. Gartenbaum.

Flor d'agua. Wasserblume. Pistia stratiotes L. var. obcordata Engl. Arareae. Heilmittel. Vide Pharm. Rundschau.

Flor a marella. Gelbe Blume. Bauholz. Bignoniaceae?

Flor de babado. , Geiferblume. Macrosiphonia longiflora Müll.Arg. Flor de babeiro. A pocvnaceae. Volksheilmittel, toxicum.

Flor de baile. Ballblume, vide Rainha da noite.

Flor de baroneza. Baronessblume. Schwammia elegans Iuss. Malpighiaceae. Steppenpflanze. Wurzel Heilmittel. Zierpflanze der Gärten.

Flor de caixão. Sargblume. Allemanda cathartica L. Allemanda angustifolia Pohl. Apocynacere. Wurzel und Blüthe.

Flor de côco. Cocosnussblume. Agaricus gardneriana Berkl. Fungi. Heilmittel.

Flor de casamento. Hochzeitsblume. Dipladenia fragrans A. DC. Apocynaceae. Knollen Heilmittel. Blüthen als Schmuck.

Flor de maio. Maienblume. Cassia aponuita Aubl. Caesaıpıneaceae. Heilmittel und Zierpflanze.

Flor de pavão. Pfauenblume. Caesalpinia pulcherrima Swartz. Caesalpineaceae. Gartenpflanze.

Flor de quaresina. Osterblume. Tibouchina Bergiana Cogn. Tibouchina stenocarpha Cogn. Tihouchina Gaudichiana Baill. Tibouchina Maximiliana Baill. Melastomaceae. Rinde Heilmittel. Blumen Farbstoff.

Flor de S. Miguel. Heilige Michelsblume. Petrea subserrata Cham. Verbenaceae. Heilmittel und Zierpflanze.

Flor de sapateiro. Schusterblume. Canavalia picta Mart. Papilionaceae.

Flor de veado. Rehblume. Forsteronia Gardneri Müll. Arg. Apocynaceae. Heilmittel.

Flor de velludo. Sammtblume. Ligeria gesnerifolia Hast. Gesneriaceae. Heilmittel.

Folho de bolo. Klossblatt. Platysciamus Regnelli W. Miconia Chamissonii Naud. Melastomaceae. Volksmittel. 
Folha branca. Weisses Blatt. Solanum megalochiton Mart. Solanaceae. Grünes Blatt zur Vertreibung der Hülınerläuse.

Foll a s de cagad o. Schildkrötenblatt. Vide Folha larga. (Vochysia). Folha de comichão. Juckblatt, vide Bucha de reado.

Folha de costa vide Folha de fortuna.

Folha de figado. Leberblatt. Acanthaceae. Volksmittel.

Folha do diabo. Teufelsblatt. Solanum Peckoltii Dam. Solanaceæ. Früchte u. Bl.

Folha de fonte. Quellenblatt. Philodendron cordatum Kth. Araceae. Ph. Rdsch., 1892, p. 281.

Folha de fortuna. Glücksblatt. Bryophyllum calycinum Salisb. Crassulaceae.

Folha de figado. Leberblatt. Ebermaiera elegans N, ab E. Acanthaceae. Heilmittel.

Folha grossa. Dickblatt, vide Sayao.

Folha de inferno. Höllenblatt (zufolge der vielen Stacheln), vide Folha de diabo.

Folha de lagarto. Eidechsenblatt. Casearia melliodora Eichl. Flacourtiaceae. Heisst auch Flor de mel. Ber. d. D. Ph. G., 1899. Schlangenantidot.

Folha larga. Breitblatt. Vochysia elliptica Mart. Vochysiaceae Nutzholz.

Pterocarpus violaceus Vog. Papilionaceae. Bauholz.

Alcornea triplinervia var. Janeirensis Müll. Arg. Euphorbiaceae. Heilmittel.

Vide auch Angelim rosa und Cajá do mato.

Folha larga do campo. Steppenbreitblatt. Vide Colher de vaqueiro. Folha miuda. Kleinblatt. Psychotria sessilis Vellos. Rubiaceae. Heilmittel.

Folh a de pagé. Blatt des Heilkünstlers der Indianer. Vide Fedegosa. Folha santa. Heiliges Blatt. Xanthosema auriculatum Reg. Araceae. Killmeyera speciosa St. Hil. Ternstroemiaceae.

Folha de Santa Anna. Vernonia macrophylla Less. Compositae. Heilmittel.

A bkürzungen. Ph. Rdsch. New York-Pharmaceutische Rundschau v. Dr. Fr. Hoffmann. New York.

Ph. Rev. Milw. - Pharmaceutical Review v. Dr. Edward Kremers, Milwaukee.

Ph. Arch. Milw. - Pharmaceutical A rchires v. Dr. Edward Kremers. Milwaukee.

Arch. d. Ph. - Archiv der Pharmacie des Norddeutschen Apotheker-Vereins.

Ber. d. D. Ph. G. Berl - Berich te der Deutschen Pharmaceutischen Gesellschaft, Berlin.

Ztschr. d. Oestr. A p.-Ver. - Zeitschrift des Allgemeinen Qesterreichischen Apotheker. Vereins. 
Folha de urubú. Aasgeierblatt. Philodendron laciniatum Engl. Araceae. Ph. Rdsch., 1892, p. 282.

Folha de viola. Miconia gratissima Bth. Melastomareae. Als Theeersatz.

Folha de vintem. Pfennigsblatt, ist in den Nordstaaten Benennung für Acarizoba.

Formgueira. Ameisenkrau. Triplaris noli me tangere Wedd. Polygonaceae. Ph. Rdsch., 1895, p. 95.

Forno. Ofen. Victoria amazonica Planch. Nympheaceat. Ber. d. D. Ph. G., 1897, p. 284.

Frecho de capoeira. Waldpeil, vide Assa peixe.

Frei Jorge. Cordia alliodora Cham. Cordiaceae. Heilmittel.

Friena und Frigúa. Fyngonium Vellosianum Schott. Araceae. Ph. Rdsch., 1892, p. 281.

Froco. Flockenkraut. Lycopodium cernumm Vell. Lycopodiaceac. Heilınittel.

Foca cheiroso. Wohlriechendes F. Microlicia graveolens DC. Melastomaceae. Heilmittel.

Fructo amargoso. Bittere Frucht, abführend. In Minas.

Fructo de Arára. Papageienfrucht. Vide Anda-assú.

Fructo de burro. Eselsfrucht. Capparis flexuosa Vellos. Capparidaceae. Ber. d. D. Ph. G., 1898, p. 45.

Fructo de cachorro. Hundsfrucht. Excaecaria liaematosperma Müll. Arg. Euphorbiaceae. Giftig. Vide auch Jasmin do mato. Fructo de caxinglé. Eichbörnchenfrucht. Nectandra Srhottii Meisc. Lauraceae. Ph. Rev., 1897.

Fructo de Conde. Grafenfrucht. Anona obtusiflora Tuss. Anonaceae. Ber. d. D. Ph. G., 1897, p. 456.

Fructo de Conde do mato. Anona acutiflora Mart. Anonaceeae. Ber. d. D. Ph. G., 1897 , p. 454.

Fructo de Condessa. Gräfinfrucht. Anona squamosa L. Anonaceae. Ber. d. D. Ph. G., 1897, p. 457.

Fructo de Cutia. Vide Anda-assú und Sapucainha.

Fructo de Gambá. Gambafr. Solanum aurantiacum Sendt. Solanaceae.

Fructo de gentio. Indianerfr. Cayaponia hirsuta Cogn. Cucurbitaceae. Abführmittel. 
Fructo de Jacù. Jacùvogelfr. Duranta Plumeri Iacq. Verbenaceæ. Fructo dos Jesuitas. Vide Buchinha.

Fructo de lobo. Wolfsfrucht. Solanum grandiflorum Ruiz et Pav. var. pulverulentum Sendt. et S. iycocarpum St. Hil. Solanaceae.

Fructo de macaco. Affenfrucht. Clavija macrophylla Miq. Myrsinaceae. Moutabea guianensis Aubl. Moutabea longifolia Popp. var. organensis. Vide auch Banana de macaco. Ph. Rdsch., 1892, p. 282.

Fructo de macuco. Macucohuhnfrucht. Licania glabra Mart. Licania heteromorpha Bth. Rosaceae. Chrysophyllum macoucou Aubl. Sapotaceae.

Fructo de morcega. Fledermausfr. Artanthe geniculata Miq. Piperaceae. Ph. Rdsch., 1894, p. 286.

Fructo de pao. Brodfrucht. Artocarpus incisa L. Urticaceae. Ph. Rdsch., 1891, p. 220.

Fructo de pavao. Pfauenfrucht. Schmidelia edulis St. Hil. Sapindaceae.

Fructo de po mba. Taubenfucht. Erythroxylum columbinum Mart. $E$. subrotundum St. Hil. E. anguifugum Mart. E. tortuosum Mart. Erythroxylaceae. Tapirira guianensis Aubl. Anacardiaceae. Ber. d. D. Ph. G., 1898, p. 157.

Fructo de pombo do campo. Myrciaria tenella Bg. Myrtaceae. Fructo de sabao. Seifenfrucht. Sapindus divaricatus St. Hil. Sapindaceae.

Fructo de tucano. Pfefferfiesserfr. Erythroxylum campestre St. Hil. Erythroxylaceae.

Fruita. Süsses Getränk mit Capsicum gewürzt.

Fruteira de burro. Eselsfruchtbaum. Uvarı trigyna Mart. Anonaceae. Capparis pulcherrima Iacq. Capparis flexuosa Vellos. Capparidaceae.

Fruteira de cachorro. Hundsfruchtbaum. Cupania marginata Camb. Sapindaceae. Arch. d. Ph. (1862), Bd. 110, p. 134.

Fruteira de perdix. Rebhuhnfruchtbaum. Byrsomina variabilis Tuss. Malpighiaceae.

Fuba. Benennung für Maismehl.

Fumo. Taback. Nicotiana tabacum L. N. brasiliensis Lk. et Otto. Solanaceae. 
Humo bravo. Wilder T. Solanum Vellosianum Dun. S. auriculatum Ait. Solanaceae. In Cearà Chamissoa macrocarpa HBKth. Amaranthaceae; Ph. Rdsch., 1895, p. 91. Polygonnm hispidum HBKth. Polygonaceae; Ph. Rdsch., 1895, p. 34. In Pernambuco Elephantopus angustitolins Sw. E. scaber L. Compositae. Arch. d. Ph. (1860), Bd. 102, p. 165.

Fumo do caboclo. Indianertaback. Nicotiana Langsdorffiii Weinm. Solanaceae.

Fumo do mato vide Herva de Collegio.

Furrundú. In S. Paulo eine Dessertspeise von Citronat und Ingwer.

Gabebeú. Heilmittel in Minas.

Gabinabo. Campomanesia aurea Bg. Myrtaceae. Frucht.

Gachipâes. Vide Pupunha.

Gachi-rama. Vide Cachirama.

Gafanhota. Heuschrecke, vide Raiz de cobra.

Gaibi-pocaiba. Vide Abaremotemo.

Galhas de almessegueira. Elemigalläpfel. Protium ovatum Engl.

Burseraceae. Ber. d. D. Ph. G., 1898, p. 440.

Gajurú vide Goajerú.

Galimetta. Dipholis salicifolia A. DC. Sapotaceae. Frucht.

Galipâes. Vide Pupunha.

Gallinha choca. Bruthenne. Erythoxylum suberosum St. Hil.

Erythoxylaceae. Rinde Farbstoff.

Gamelleira. Wannenbaum. Urostigma Doliarium Miq. Urticaceæ. Gamelleira branca. Weisser W. Doliarin, Specificum bei Anchylostema. Arch. d. Ph. (1858), Bd. 94, p. 364; Ph. Rdsch., 1891, p. 166.

Gamelleira in Minas. Brosimum discolor Schott. Urticaceae. Ph.

Rdsch., 1891, p. 219.

Gamelleira brava. Wilder W. Urostigma eximium var. glabrum

Miq. Urticaceae. Ph. Rdsch., 1891, p. 219. Clusia Burchelli Engl.

Guttiferae. Ber. d. D. Ph. G., 1897, p. 240.

Gamelleira trepadeira. Kletternder W. Vide Mata paò.

Gangao. Ein atropischer Maiskolben.

Gang o u zù. Vide Coqueiro cauassù.

Ganha-acolà. Centropogon surinamensis Presl. Lobeliaceae. Blätter Heilmittel.

Ganha saia. Vide Apanha saia. Ber, d. D. Ph. G., 1897, p. 103. 
Garaita. Chrysophyllum flexuosum Mart. Sapotaceae. Rinde Früchte, Holz.

G arajuba. Terminalia acuminata Fr. All. Combretaceae. Holz für Wasserbauten. Corrumpirte Tupibenennung von Muyra-yuba, gelbes Holz.

Garapa. Benennung des gegohrnen Zuckerrohrsaftes, entlehnt der Tupinambasprache, welche ein gegohrnes, mit Waldhonig versüsstes Getränk benennen.

Garapa. Dialium divaricatum Vahl. Caesalpineaceae. Bauholz. Vide auch Guarapeapunha.

Garapiroca. Noch nicht bestimmtes Bauholz.

Gara-qu y inh-assú. Vide Càaxia.

Gararóba. Bauholz.

Garauna. Vide Barahuna.

Garauna ruiva. Sclerolobium paniculatum Vog. var. rubiginosum Bth. Caesalpineaceae. Vorzügliches Bauholz.

Garfuana. Centrosema dasianthum Bth. var. pubescens Wawra.

Papilionaceae. Heilmittel.

Garubú. Vide Guarubú preto.

Garuiba. Vide Gurijúba.

Garupé. Aus der Asche dieses Baumes bereiten die Indianer Salz zur Speise.

Garyroba. Vide Paty amargoso. Corrumpirtes Guaraniwort, von Naryrob-Palmknhl, rob-bitter.

Gaviroba. Cocos plumosa. Hook. Palmae.

Gazerú. Vide Abajerú.

Genciana. Enzian. Lisianthus uliginosus Grieseb. |Gentinaceae. Genciana da terra. Lisianthus pendulus Mart.

Genciana do sertão. Lisianthus coerulescens Aubl. Ges., 1899. Gendiroba. Vide Nhandiroba.

Geneúna. Vide Canna fistula und Mari-Mari.

Genipabeira. Genipa americana L. Rubiaceae. Frucht essbar. Genipapeira do muto. Genipa brasiliensis Mart. Rubiaceae.

Zeitsch. d. a. öster. Apt.-Ver., 1896, p. 227.

Genipapo. Frucht des Genipabaumes.

Geniparana. Falscher Genipabaum. Japarandiba augusta Ok. Genipua-rana. var. brasiliensis Rg. Lecythidaceae. Früchte Genuparana, J und Holz. 
Gequirity. Vide Jequirity.

Geratacaca. Vide Manacã.

Gerebita. Vide Mandurèba. Auch Benennung der Neger für 'Zuckerbranntwein.

Gerema. Vide Jurema.

Gergelim. Sesamum orientale L. Bignoniaceae. Kultivirte Oelpflanze.

Gergelim bravo. Wilde G. Crotalaria stipularia Desv. Papilionaceae. Samen.

Geribà. Corrumpirtes Guaraniwort, von yary-klebrig, úa-Frucht.

Vide Baba de boi. Ph. Rdsch., 1889, p. 90.

Gericò. Vide Ambar vegetal. Vide Ph. Rdsch., 1894, p. 240.

Geriquiti. Vidi Olho de pomba.

Gertrudes. Apium ammi Urb. Umbelliferae. Kultivirt.

Gerumar̀̀. / Capia centijuga Wawra. Caesalpineaceae. Rinde, Gerumari. $\}$ Fruchtpulpe und Samen.

Gervão. Vide Aguará-ponda.

Gervã de folha grande. Grossblättriger G. Bouchea pseudogervão Cham. Verbenaceae. Heilmittel.

Gessará. Vide Assai. Ph. Rdsch.. 1889, p. 167.

Getica. Vide Batata doce.

Gicão. Serpaea cearensis Fr. All. Cruciferae. Ber. d. d. ph. Ges., 1897 , p. 288.

Gigógo. Nymphaea Rudgeana G. F. W. Meyer. Nymphaeaceae.

Ber. d. d. ph. Ges., 1897, p. 285; Arch. d. Ph., 1860, Bd. 102, p. 168.

Gigogo in Rio de Janeiro. Nymphaea dentata Heut. Nymphaeaceae. Gigoia. Vide Canna do brego (Costus). Ph. Rdsch., 1893, p. 289. Gilbarbeira. Ruscus aculeatus L. Smilaceae. Verwildert. Ph.

Rdsch. 1893, p. 81.

Giló. Solanum melongona L. Solanaceae. Frucht, Gemüse. Gindiroba. Vide Nhandiroba.

Gingeira brava. Wilder Kirschbaum. Prunus sphaerocarpa Sev. Rosaceae. Zeitsch. d. a. öster. Apt.-Ver., 1865, p. 393; Arch. d. Ph., 1858, Bd. 94, p. 364.

Gingeira de Jamaica. Malpighia glabra L. Malpighiaceae. Gingeira da terra. Solanum pseudocapsicum L. Solanaceae.

Früchte. 
Gingibre. Ingwer. Zingiber officinalis L. Zingiberaceae. Kultivirt. Vide Ph. Rdsch., 1893, pp. 287, 289.

Ging ibre d'o urado. Goldingwer. Curcuma longa L. Zingiberaceae.

Kultivirt. Vide Ph. Rdsch., 1893, pp. 287, 289.

Ginguba und Ginhyba. Negerbenennung von Amendoim.

Ginjá. Vulgärname für Kirsche.

Gingúiba. Heilpflanze in Bahia.

Gipio. Heisteria brasiliensis Engl. Olacinaceae.

Gipio-rana. Bauholz in Amazonas.

Gique und Giqui. Vide Acaya. Ber. d. d. ph. Ges., 1898, p. 153.

Giquirity. Vide Jiquirity.

Girasol. Sonnenblume. Helianthus annuus L. Compositae. Kultivirt. Giriba. Cocos Romanzoffiana Cham. Palmae. Ph. Rdsch., 1888, p. 91.

Giiribú. Vide Jureva.

Girimato. Vitex Gardneriana Schauer. Verbenaceae. Heilmittel. Girimú. Vide Abobora.

Girimú paó. Vide Paó de girimú.

Giruba. Vide Giriba und Girúva.

Giruva. Cocos acrocomoides Dr. Palmae. Ph. Rdsch., 1888, p. 89. Gitahy. Caesalpineaceae. Nutzholz in Alagoas.

Gitahy amarello. Gelbe G. Thomasia pieudolutea Fr. Allem.

Büttneriaceae.

Gitirana de florbranca. Weissblütige G. Prevostia spectabilis

Meiss. Convolvulaceae.

Gitirana de leite. Milch G. Ibatia quinquelobata Fourn. Asclepiadaceae. Heilmittel. Vide auch Bordãosinho.

Gito. Guarea alterans C. DC. Meliaceae. Früchte und Rinde.

Gito in Pernambuco. Guarea tuberculata Vell. var. purgans DC.

Meliaceae. Rinde und Wurzel.

Gito utauba. Vide Assafrôa.

Go a jer ù. Papageienfrucht (Tupy). Chrysobalanos icaco L. Rosaceæ.

Bl. Frucht und Wurzel.

Goarana. Vide Gurana.

Goerana. Chrysophyllum perfidum Fr. Allem. Sapotaceae. Samen, giftig. Bauholz.

Gogo. Negerbenennung für Carurú Bahiana. Vide Ber. d. d. ph. Ges., 1898, p. 281.

Goiaba. Frucht der Guiabeira. 


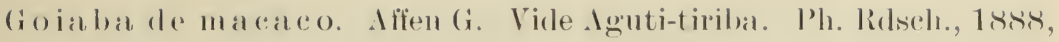
p. $(i$.

(iobabada. Marmelada der Goiaba.

(ioiabeira. Virle Guiabeira.

(ioiabinlıa. Vicle Iraça do mato.

(ioiabi-pocaca-iba. Mimoseare. Amazomas.

Goitoroba mol Goiti-turuba. Vide Iguti tiriba.

Golpho. Lisianthus purjurestems Aubl. Gentianderale. Ber. d. d. ph. Ges., 1899.

(iolplo grande. Grosser (i. Virle Gigogo.

Gomma de lagrymas. Hary ron draxima.

(

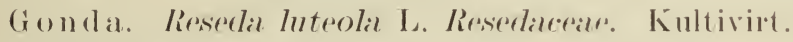

Gonó. Vide Tayuyá de quiabo.

(iоnи. Vicle Tayuyá miuda.

(ionzalo.

Gonzalo Alves. Iide ('hibatan. Ber. d. d. ph. (ies., lsas, (ionzalo do mato. I p. $16: 3$.

Gorana-timbó. Von Tupy-uarana-(ietriunk, timbo-(iift. C'amptosema pinnatmm Bth. Cassalpinealrearr. Rincle Heilmittel. Wurzel Fisclogift.

Gorarema. Gallisial Gorazemia Moq. I'hitolateraterate. P'h. Relsch., 1895, p. 21..

(iororóba. Vide Araribà rosa.

(ioya-ibira. Virle Iraribà.

Goyana-timbó. Vide Gorana timbó.

(ioyti. Vide Oity.

Gragojó. In den Nordstaaten Benennung fïr Abobora d'agua.

Grama. Quecke. Triticnm repens L. Gramineae. Vide Ph. Rolsch., 1894, p. 109.

Grama de Maranhao. Vide Tabaquinha.

(i r a ma de Pará. Vide Sap̈̈.

Grama da praia. Küstengras. Stenotaphrmm gliblum Trin. var.

multiflormm Gramineae in Bahia. Grmmothrix nervosa N. ab. E.

Viehfutter.

Grama ruiva. Rothes Grasblatt. Machaerium brasiliense Vog.

Papilionaceae. Bauholz. Blätter Viehfutter.

(iramonde. Ville Guarabú preto.

Giranonde piqueno. Melastomaceale. Nutzhol\%. 
Grao de bico. Kichererbe. Leguminosite. bauholz. Firer arietimum L. Papjilionareale. Kultirirt.

Grao de carallo. Vide Piquia.

Grao de gallo. Hahnhoden, zufolge der Fruchtorösse. C'eltis glırocarfua Mart. Irticareae. Vide P'h. Rdsch., 1892, p. 3.).

Coronema spinosa Reiss. Rhamnaceale. Frucht essbar.

In Minas, Lucuna tortal A. D( '. Silpotaceare. Ph. Rdsch., 1s8s, p. 7.

In Parà, Chinchona raprifolia Lacerd. Rubiaceae.

Vile auch Vara-apea.

Grao de gallo dos grandes. C'eltis aculeatasw. Crticareate. P'h.

Rdsch., 1892.

Cordia platryhrlla siteud. Cordiacrae. Frucht essbar.

Grao de macaco. Affenhoden. Montabea gruanensis Iubl. Ehonaceae.

Grao de porco. Schweinshoden. Phrsocalımna scaberinum Pohl. Lytracpate. Cordia grandifolia A. DC .: Cordia hebecarlua DC. Corrliaceae. Vide auch rega machado.

Groas de chumbo. Bleischrot. Vide Banua. Aehnlichkeit der samen. Graos de chumbo grosso. Grober Bleischrot. Cardiospermum Corindum L. Sapindareate.

Grapia punha. Apulea praterox Mart. Catesalpineareate. Bauholz. Grapiapunha branca. Apulea polrgama Fr. Allem. Caresalpineaceae. Bauholz.

Grapihi. Bauholz in stat. Catharina.

Grauna. Vide Barahuna.

Gravatá. Vide Caraguatá. | Ville Hh. Rolsch., 189.j, Gravatá assí. Vide Caraguatá assí. f p. 2:37.

Graratá de agulha. Vicle Inanas de aǧulha. Vide Ph. Rdsch.. 1895, p. 2:39.

Gravatá bravo. , Brumelia fistusosil Lindl. Bromeliaceate. Graratá do mato. i Vide Ph. Rdsch., 1895, p. 237.

Gravatá de rede. Allanas sativus schult. var. bracteatus Lindl. Bromeliacede.

Gravatá de tingir. Aechmeal tinctoria Metz. Bromeliaceae. Ph. Ridsch., 189.5, p. 239.

Graviola. Anona Cearensis Barb. Rodrg. Anonaceae.

Graxa. Wichse. Vide Mimo de Venues.

Greuhitinga. Bauholz in Paraná. 
Gritadeira. Schmerzplanze. Psychotria rigida Willd. var. gennina Miill. Arg. Ruhbiareale. In s.. P'aulo I'syrehotrial rigirlal Willd. var. hirterlla Miill. Arg. Rullialreare.

(iritadeira do campo. Steppen s. Psirehotrial rigirlal Willd. var. strepens Mïll. Arg. Rulliacrate.

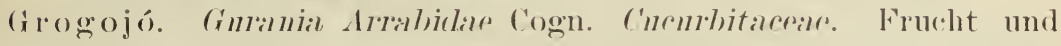
Winzel.

Groselheira da India. Indische Stachelberere. Vide Bilimbi. Ber. 1. d. ph. (ies., 18!99, p. 4!).

(irujahi. Apeiba membranarma simue. Tiliarpare. Ber. d. d. ph. (ies., 1898, p. 284.

(irlumb.

1 Aspirlosperma mompormm Miill. Arog. Aporyarease. (ir umamel. f Baulolz. Milch Heihnittel.

Gir 1 ma a mé. Vide Mata olhos.

(ir umarim.

Grumary. i Vicle Páó setim.

(irumixama. Frucht von:

Grumixameira. Nach Dr. Martius corrumpirtes Tupywort von curumin-kleine, cama-Brustwarze; nach Beaurepaire Rohan. Yba-mixana-Schwarz-rothe Frucht. Stenoralyx brasiliensis Bo. Myrtaceate. Frucht ist gross und rund, dunkelroth, keine Aehnlichkeit mit einer Brustwarze. Wohlschmerkend.

Grumixama branca. Weisse (x. Stenocalyx brasiliensis var. leneocarpus Bg. Myrtaceale. Frucht weiss, wohlschmeckend.

Grumixama brava. Wilde G. Stenoralyx silvestris Bg. Myrtacere. Grundeuva. I Astroninm nonndenva Eingl. Anarardiareate. Ber. d. (irundiúba. ) d. ph. Ges., 189s, p. 16:3.

(iuabŕbía. Vide Gabebeì.

Guabijù. Vicle Guabira-guassì.

Guabijù mirim. Kleine G. Engenia Itacolnmensis Bg. M!rrtareap. (iu a bijù. Vidle Guabira gouassù.

Guabi-poca-cahiba. Vide Abaremotemn.

(iu abiraba. Cordia.glabrata A. DC. Corliaceae. Frucht und Holz.

Abbevillea maschalantha var. ovata Bg. Frucht.

Guabir á-guassì. Grosse G. Corrumpirtes Tupywort: Gua-Beere, yrob-bitter. Eugenia Guabijú Bg. Myrtaceae.

Guabira mirim. Kleine G. Vide Guabiroba merim.

(iuabiroba. Tupywort. Süisse Beere. Camponanesia rorymbosa

Bg. Myrtilceae. 
In Minas Camponlanesia olversa Bg. Mrrtareale.

In Parà Eugenial mivrohalana DC. Mrrtareale.

In Pernambuco Campomanesial ciliata Bg. Mrrtalceate.

In Rio Grande do sul Campomanesia reticulata Bg. cf. C. Varlilbilis Mart. et. C. craneal var. orata Bg. Mrrtaceae.

In s. Paulo Campomanesia guazumatolia var. rubiginosia Bg. Mrtaceae.

Gu a biroba ass ù. Grosse G. Camponnanesia repanda Bg. Mrrtacear. Guabiroba brava. Wilde G. Campomanesia xanthocarla Bg. et

C. desertorum Bg. Mrrtaceae. Frucht sauer und bitter.

Guabiroba de eachorro. Hunds G. Abberilea Ginabiroba Bg. Mirtaceae.

G u a bi roba do campo. Steppen G. C'anpomanesia microcilpra Bg. Mrrtaceae: $r^{\prime}$. vireprens. Bg.: ('. Klotschiana Bg. Mrrtareate. Vide auch Gabinaba.

(iuabiroba doce. Süsse G. Abberillea Fenzliana var. intermerlia Bg. Mrrtaceae.

Guabiroba felpuda. Haarige G. Campomanesia discolor var. oppositifolia Bg. Mrrtaceae.

Guabiroba grande. Grosse G. Campomanesia rrenata Bo. ('. transalpina Bg. et Abberillea Fenzliana Bg. Mrrtaleale.

Gu a biroba g u assù. Vide Guabira-guassù.

(iuabiroba J a mbó. Rosenapfel (i. C'ampomanesia gutazunaefolia

Bg. Mrrtaceae.

(i u a biroba lisa. Gilatte G. Cannpomanesia obscura Bg. Mrrtaceir.

In Minas Campomanesia obrersal Bg. Mrrtareaze.

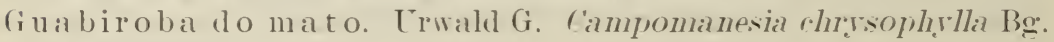
Mrrtarcane.

Guabiroba mirim. Kleine G. Campomanesia aprisa Bu. et Mrrtus mulcronatum Camb. Mrrtareae.

In Rio Grande do sul Mrrtus mucronatus Camb. var. opaca Bg. Mrrtaceate.

Guabiroba roxa. Violetrothe G. Camponanesia fusra Bg. Mrritareaze.

('uabiroba do sertão. Wiisten (i. Campomanesia multiflom Bg. Mrrtaceae. Heisst auch Araca branea.

(i u a bir ú. Vide Guabira assì.

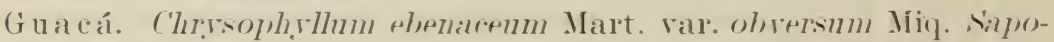
taceile. 
(i u a cá assì. Grosse (i. Protimm Riecleliammm Engle et P. marro-

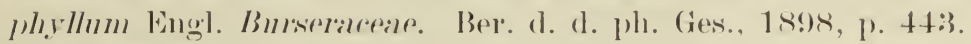

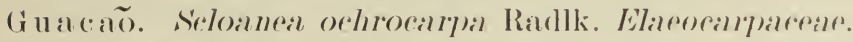

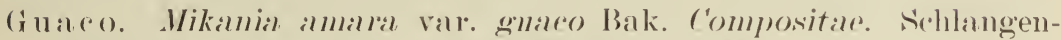
antidot. Areh. d. Ph, 1857, B3. 91, p. 108.

(iuacury. Scheelia minceps valr. ('ormmbensis Barb. Rodr. I'almar. corrumpirtes Tupywort: F́a-Frucht, kuriv-\%apfen.

(xuadua. Gmadua angustifolia Kth. Graminfare. Rohr. Ph. Rolsch., 1894, p. 169.

Guadua mirim. Kleine G. Guadua marrostarlyyal Rupr. Gramimeap.

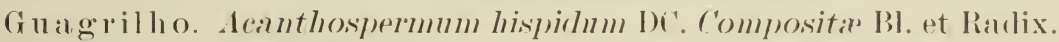
Guaguassí. Vide P’alha branca. Ph. Relseh., 1889, p. 1:3:3.

Guaiavita. Combretum framgulifolimm KBKth. ('ombretarear.

(iuaibi-poeaiba. I'ithecolobium salmam var. arentifolial Bth. Mimoseae.

(iuaimbe. I'hilodendron squamifermm Poepp. et var. arerifirum

Engl. Araceate. Vide Ph. Rdsch., 1892, p. 282.

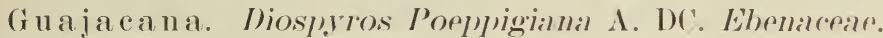

Guajaná-timbo. 'Tupybenennung' fïr Anil.

Gua jará. Viıle Garajúba.

Guajara branca, mirim und vermelha. Weisses, kleines und rothes $\mathrm{k}$. Bauholz in Maranhao und P’à̀.

(iuajará-timbó.

Guajarahy.

(u i jerí.

\} Vicle (ioajerú.

Guajubeia. Baulıolz in Rio Crancle do Sul.

Gu a juvira. Myrticeae. Bauholz.

Guamarim und Guamerim pintado. Cusparia ovatia Engl.

Rutaceae. Vicle auch Sabia-cica. Ber. (1. 1. ph. Ges., 1899, p. —. Gu a mbé. Vide Guiambé.

Guam ix inga. Vicle Chupa ferro. Vicle Ber. (1. d. ph. Ges., 189!), p. - . Guanabano. Vide Taqueira dos pobres.

Guanandy. Vide Anany.

Guando. Cajanus indicus Spreng. Papilionaceate. Nahrungsmittel. Guaparaiba und Guapariba. Vide Mangue vermelho.

Guapeapunha. Vide Grapiapunha.

Guapeba. Frucht. I Lucumal lanrifolia 1. I)C. Silpotaceate. P'h.

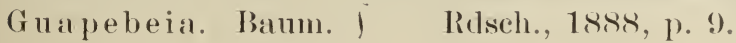


Guapera. Feuillea albitlora Cogn. et F. trilobata cogn. Cucurbitaceile.

fiuapera legitima. Aechte (i. H.rpanthera Guaperal Manso. ('ncurbitaceine.

Guapicopaiba. Vide Flor de Maio.

Gua poranga, Gua puronga, Guapurunga. Marliera tomentosa Camb. Mertareae.

Guapuam. Bauholz in Paranà.

Guapur. Stizophyllum perforatum Miers. var. dentata Bur. Bignoniaceas.

Guapuica. Engenia Vantheriana Bg. Mrrtaceae.

Gua rabú. Viđe Guarúbú.

Guaracica. Lucuma fissilis Fr. Allem. Sapotareas. Bauholz.

fuaraitì und Guaraitó. ('hrosophrllum Cisueiri Fr. Allem. Silpotaceae. Bauholz.

Guarajúba. Vide Garajúba.

Gua rand. Guaranapaste aus den caffeinhaltigen Samen der Guaranàpflanze. Genussmittel.

(iuaraná-ura. Guaranapflanze. Panllinia sorbilis Mart. Sinpindaceap.

Guaranhem. Vide Buranhem. Ph. Rdsch., 1899, p. 30.

fua rantan. Matarba arborescens Radlk. Sapindaceae. Bauholz. Gua rapa. Vide Garapa.

Gua rapa ré. Weinmannia linta Mart. Saxifragaceale.

(iuar a parm vermelho. Bignoniaceae. Baulıolz in Parani.

Guarapeapunha. Vile Grapeapunha.

(iuaraquim. Ville Herva moura.

(iuara-puymia. Fitrauch in Ilagoas. Rinde Wurmmittel.

(iuararema. Vide Ibirarema.

Guar riroba. Vide Guariroba do campo.

(iuararoba. Vide Araribá.

(i uarassahy. Moldenhanera floribunda Nichrad. C'assalpinealcear. Bauholz.

Guarataia do morro. Vide Arapoca anarella.

Guaraúna. Vicle Barahuna.

Guarauna luiva. Vide Garauna ruiva.

(i ua rdião. Melothria punctatissimal Cogn.: M. Anminensis Gaertn.

Cucurbitaceap. Samen und Wurzel.

(i ua lé. Vide Issafrôa. 


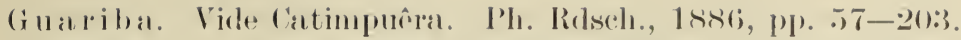

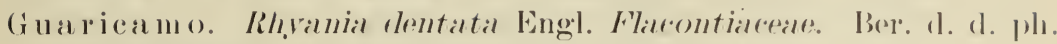

(ies., $18999, \mathrm{p}$. -

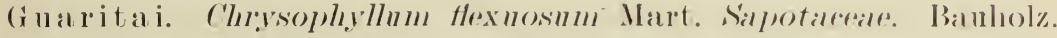

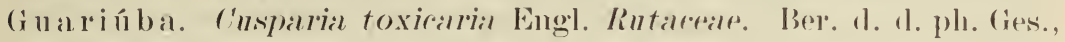

$1899, \mathrm{p}$. -

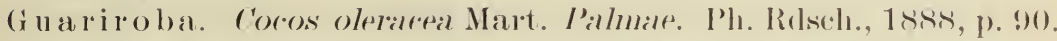

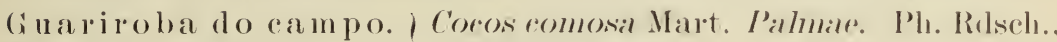
(iuarirobinha. $\quad$ f 1888, p. 90.

(

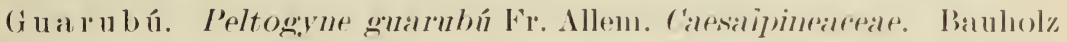
und Farbstoff.

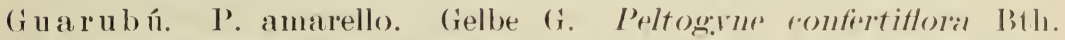

Cassalpineaceate. Bauholz.

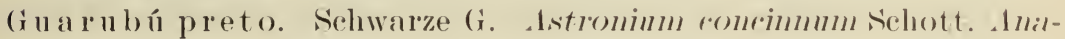

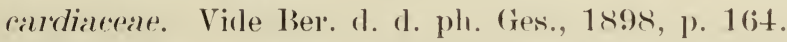

(i u a rub ì rogo. Fuchsiothe (x. Lallnareate? Baulıoly.

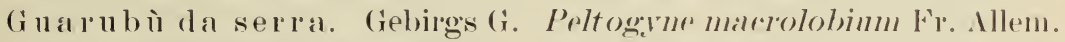

Causalpineaceare. Bauholz.

(i u a r íma. Vide Lrubá de caboclo.

Gu a rum ima. Serjania rnspirlatal ('anb. Nilpintlatrale. Fischgift.

Guarpêma. Vicle Urupêma.

Guatambí. Vicle Pequia amarella.

(tuaviroba und (x. do ceimpo. Vide Butia do campo).

Guaxica. Rhopala brasiliensis Polıl.; R. tomentosia l'olıl. Proteaceate. Bamholz.

(ivaxima. Vicle Carrapicho. Ber. 1. 1. ph. Ges., 1898, p. 282:

Arch. d. P'h., 18:5:3, Bd. 12:3, p. 114.

Guaxima branca. Weisser (i. Helicteres P'ermamlnoensis drud.

Malvaceale. Faseistoff.

Guaxima macho. Männlicher (i. Vlena lobata ('av. Malvaceap. Faserstoff.

Guaxima mangue. Manga (i. Hibiscus I'ernammlncensis Arrud.

Malvaceae. Faserstoff.

Gux ima do mato. Vide Guaxima hranca.

Guaxima preta. Schwarze G. Pharmacosycea radnlil Miq. Lrticaceae. Ph. Risch., 1891, p. 16.).

Guaxima roxa. Violetrothe G. Vide Guaximo macho.

Guaximba preta. Vide Guaxima preta. 
(iuaxindiba. Kedia brasiliemsis Barb. Rodr. Malvareate. Rinde off. (i ua xinduba preta. Vide Guaxima preta.

(i u a y a can. Lafoensia speciosa DC. Lythrareate. Heilmittel.

Gua yambé. Heilmittel in Amazonas.

Guazúma. Guaznma ulnifolia Lam. var. tomentosal Sieh. Sterenlialceate.

Guazuma macho. Männliche (i. Grazuma nhmifolia Lam. var. tomentilla sch. Sterculialceate.

(i 1 a zuma torcida. Docht (i. Gnaznma nlmifolia Lam. Varr. Velutina sch. Sterculiaceae.

Guela le pato. Entenkehle.

(ivembé. Vile Imbá.

( Guereroba. Aspidosperma australe Miill. Arg. Apocynaceae. Banholz und Rinde. In Maranhao heisst: Cascal amargosa-Bittere Rincle.

Guereroba de remo. Ruder G. Aspidosperma anomalum Müll. Arg. Apocinaceate.

(iuiabarı. Virle Bago da praia. Ph. Rolsch., 189.), p. 3.5.

Guiaba da or. (Guayaba. Marmelade von der Frucht I'siclinm guayava Radd. In den Nordstaaten heisst: Doce de arassi und in Portugal: Hoce de tijolo-Backsteinconfekt.

(iniaba-rana. Falsche (i. Psidinm acutangulum I)(. Myrtaceate. (i u iabé-ambé. Kräitziger G. Psidinn Paraense Bg. Myrtalceate.

Guiabeira und Guyabeira. Gajavenbaum. Corrumpirtes Guaraniwort. Psidium guyala Radd. Myrtaceae. Arch. d. Ph., 185:3, Bd. 12:3, p. 114. In S. Paulo wird Araca felpudo und in Pernam. buco Araça piroco, auch Guyabeira benannt.

Guiabeira brava. Wilde (A. Psidinm densicommun Mart.; Myrcia allceps Bg. Myrtaceae.

Guiabeira de cutia. Iguti (i. Eugenia Mikaniana Bg. Myrtaceie. Guiabeira de macaco. Affen G. Vide Guitiroba-assì.

Guiabeira do mato. Vicle Guiabeira brava.

(iviabeira de paca. Paka G. Mrrcia Alagoemsis Bg. Mrrtalceate. (iuiabeirana. Vide Guiabà-rana.

Guiabinha. Myrtaceae. Nutzholz.

Gu iambè. Philodenclron squamifermu Poepp. Araceate. P'h. Rdsch., $1892,1) .2 \times 2$.

(iuiabi). Virle (iniragombò. Aroh. (l. Ph., 1857, 91, p. 110; 1860, 102 , p. 16.5 . 
Guiaruna. Virle Barahmun.

(inimbis.

(inimbi-rana. I Vide Imbr da maia.

(iuimbì da praia.

finimberana. Vicle anch folla da fonte.

(iuiue contra cobra. I'olygala fimbriatil A. IY. Bennet. Molygalacear. Wmoel sichlangenantiulot.

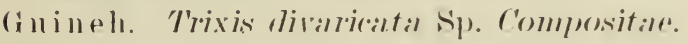

(inimlla. Vide (2nina de l'ermambureos.

(illilis. Vicle (invia tepoty.

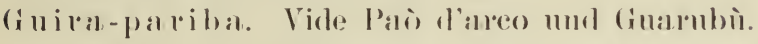

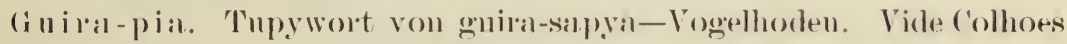
de grallo. P'h. Rals(-J., 18!)2, p. 34 .

(iniso de cascavel. Klapperschlangenklapler. Virle Cascavel.

(xuiti mol (initi-guasusu. Virle Oiti.

Guiti mirim. Kleine (i. Virle Oiti da plaiis.

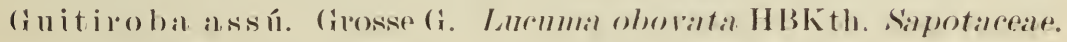

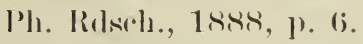

(ruity. Vide foureto de silbiño.

(iuity-rogra. Virle ()iti-('mor).

(iuity-iba und (iuity-toloba. Vinle Aguti-tiriba. Ph. Ralseh.,

$18 \times 8$, ]). (i.

(iniya-asisí. Virle Axi.

(iuiyabada. Virle (iniabarlas.

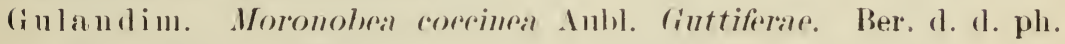
(ies., $1 \mathrm{~s}) \mathrm{T}, \mathrm{p}$. 24t.

(i) ranhem. Vinle Buramhem.

(iulauil. Vellosial aloaefolia Mart. Irllosiareare Faserstoff.

(iurataia poca. Vicle Arapoca amarella.

(i 11 rijúba. Vide (Auarińba.

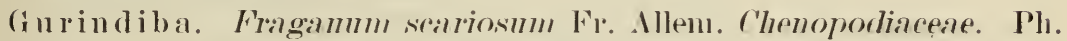

Rilsch., 1895, p. 89.

(i u r ixima. Vide Quixińme.

(iarubì. Vide Chibatan. Ber. 1. 1. ph. Ges., 1898, p. 163.

(iurupé. Moqnilea pentula. Bth. Rosaceae. Frucht essbar.

(iury. Vide Imbiri.

(iuryri do campo. Diplothemmm rampestre Mart. var. genuinum

Dr. Palmare. Ph. Rolsch., 1889, p. 111.

Guyambé. Strauch. Arch. d. Plı., 1860, 102, p. 165. 
Guyra-repoty. , Tupy. Guyra-Vogel, repoty od. tepoty-Koth. Guyra-tepoty. i Struthanthus marginatus Bl. rar. panimlata Eichl. Loranthaceae. Ph. Rolsch., 1xst.

G ypio. Vide Gipio.

Gyquirioba. Vide Jequirioba.

H a ry y. Guaranibenennung für Acumão rasteiro. Haryb-Traube, y-sehr klein. Ph. Risch., 1889, p. 92.

Herva de amor. Liebeskraut. Vile Trero.

Herva de andorinha. Schwalbenkraut. Enphorlyil roprorum Mart.

Euphorhiaceae. Arch. (1. Ph., 15.57. Bd. 8.2. 1. 9.2. Im staate

s. Paulo ist es Euphorhia brisilipnsis I anu.

Herva de anil. Vide Inil miudo.

Herva de babosa. Vide Babosa. Ph. Rdsch., 1\&9:3, p. 80.

Herva de barbonas. Pfaffenkraut. Vide Barba de relho. Ph.

Rdsch.. 1895, p. 2039.

Herva benta. Benediktenkraut. Gienm nrbannm L. Rosarpae.

Heisst anch Ilface de cordeira, Lammsalat.

Herva de bexiga. Pockenkraut. Sianvagesia tenella Lam. Sinnragesiareate. Ph. Gesch., 1897, p. 298.

Herva de bicho. Wurmkrant. Polrgonum acre HBKth. Polvgonaceate. Ph. Rakch.. 1s9.). p. 3.5: Arch. d. Ph.. 18.5:3. Bd. 12:3. p. 114 .

Herva de bicho im state s. Paulo. Vide Herva de sianta Maria. Herva de boi. Ochsenkraut. Vide Timbó de boi.

Herva de botao. Knopfliaut. Vide Lanceta.

Herva de brejo. Vide Herva de pantans.

Herva de bugre. Indianerkraut. Euphorhia romosia Vellos. Enphorliaceae.

Herra de cabra. Ziegenkraut. Euphorbia brasiliensis Lam. var. hrssopifolia Bois. Euphorbiaceae. Tödtet Ziegen.

Herva de rachos. Traubenkraut. Vide Tintureira vulgar.

Herra de callos. Hühneraugenkraut. Vide Folha de Furtuma.

Arch. d. Ph., 18.53, Bd. 123, p. 114.

Herva de canudo. Röhrenkraut. Hrptis fasriculata Bth. Lahiatae. Arch. 1. Ph., 1860, Bd. 102, p. 16:3. In Staate Bahia ist Hiptis macrostachus Bth.

Herva do canudo do campo. Steppenröhreraut. Hrptis lippioirles: I'ohl. Labliatae fromatirate. 


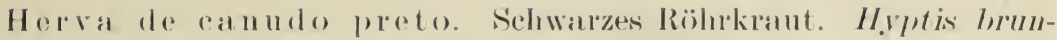
ascens Polıl. Lalliatare dromiaticale.

Herva de rapitão. Vide Acarizoba. Areh. d. Ph., 1862, Bel. 110.

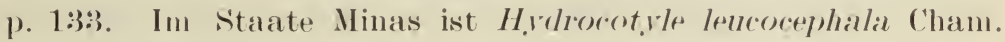
limbelliferiar.

Herva de vardo am a rello. Vide Cardo amarello. Ph. Gesch., 1898, p. 286.

Herva de ('arpinteiro. 'Kimmermannskraut. Arhillea millofolinm

1. C'ompositate.

Herva de Catinga. Stinkkraut. Vide Mrruta de campina. Areh. d. Ph., 1862, Bd. 110, p. 133.).

Herva cáuna. Blattkraut. Vide Mate.

Herva centaurea. Tausendgïldenkrant. Injanira erubescens ('ham. et schlecht. Crontianicear. Ph. Gesch., Berlin, 1899.

Herva de chumbo. Bleikraut. Avicennial alveolata Lacerela. Myosporinareate. InI Ntante Parà ist Ciassythal amerieanal Nees. Lallralceile.

Herva cirlreira. Benemung der ottionellen Melisse.

Herva cidreira hrava. Wilde Melisse. Liplpial betulatefolia HBKth.

Verbeniaceate.

Herva cidreira lo mato. Waldmelisse. Huptis seralleoloms Poit. Lalliatile. Heisst auch ('amarál-cilrilhat.

Herva cidreila da terra. Einheimische Melisse. Hyptis ralpitatal

Iacq.: H.ptis mmlnosal Salzm. var. Mikanii Sehum. Liabiatar.

In Stante Parà Lantana ranescens HBK ch. Verbenacear.

Herva de cobra. Schlangenkraut. Marsypianthes hyptoides var.

arenosil śchum. Labiatile. Mikilnial rordifolia Willd. Compositate.

Sichlangenanticlote.

Herva de cobras. Enphorbia piluliferal L. var. prormmbens Bois. Emphorliaceate.

Herva de coellı. Kaninchenkiant. Mikanial erioclarla DC. Coms. positile.

Herva de Collegio. Selunlkraut. Elrpliantopus scaller I. var. tomentosus S'chulz-Bip. Compusitate. Officinell. Arch. d. P'h., 1853, Bd. 12:3, 1. 115; 18.58, Bd. 9)3, 1. 120.

Herva ia Costa. Kiistenkraut. Srolubertia multiforal Mart. et Huce. Asclepiadiacere.

Herva da Cruz. Kreuzkraut. Vide Herva lombrigueira.

Herva do diabo. Teufelskraut. Vide Loco. 
Herva doce. Benennung für Anis.

Herva doce bravo. Wilder Anis. Errthoxylum distortum Mart. Erythoxylaceare.

Herva doce manso. Zahmer Anis. Erothoxylum mucronatum Benth. Erythoxylaceas.

Herva dutro. Rauschpflinze. Tibonchinal Martinsianal Cogn. Melastomaceate.

Herva ensete. Banauenkraut. Baccharis megalpotonica sipreng. Compositae.

Herva espia caminlo. Wegweiserkiaut. Vide Herra mijona.

Herva feiticeira. Hexenkraut. Vide Icacariba.

Herva de feridas. Wundkraut. Vide Albará (bei Albará ist Druckfehler, steht Allearí), anch ('annil latifolia Rose. ('annacear. Ph.

Rilsch., 189:3, p. 2.57; Areh. (l. Ph., 18.7, Bel. 89), p. 247.

Herva de ferro. Eisenkraut. Vide Purga de Joa Paes.

Herva de figado. Leberkraut. Vide Lingua de vacra.

Herva de frechas. Raketenkraut. Vide Cravo de defunto.

Herva de gato. Katzenkraut. Vide Narcella do mato.

Herva gorda. Fettkraut. Pilea muscosal Lindl. Irticarcear. Ph. Rdsch., 1892, p. 34 .

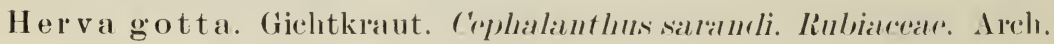
d. P’.. 18.57, Bd. 91, p. 111.

Herva grossa. Dickblatt. Virle Herva de Collegio.

Herva de Jaboti. Schildkrötenkraut. Artanthe alunci Miq. Piperacede. Ph. Ridsch., 1894, 1. 285.

Herva de impigem. Flechtenkraut. Syris latifolial Mart. Tyridaceale. Ph. Relsch., 1892, p. 104. Auch Comanditaia hat diese Benennung.

Herva de inhambú. Inhambúhuhnkraut. Virle Nhamburana.

Herva de João Feixeira. Vide Herva de s. João.

Herva de Lagarto. Eidechsenkraut. Cascartia parvifolia Willd., Casearia Clausseana Miq., C'asearia lantifolia Salzm. Flaconrtiallareae. In Bahia Caseantia oblongifolia Canb.; in S. Paulo ('asearial sylvestris Swarz. Flacontianderate. Acrh. 1. Ph., 91, p. 111 ; Ber. d. d. ph. Ges., 1 s!9, p. 171.

Herva de Lombrigas. Spulwurmkraut. Vide Arapabaca. Herva lombrigueira. Wurmkraut. Chenopodium anthelminticum L. var. glabratum Fenzl. Chenopodiaceae. 
Herva laneeta. Vide Lanceta. Laturettenklant. In S. I'anlo ist

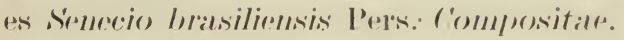

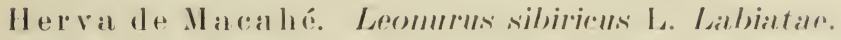

Herva contra mal de ventre. Ville Herva lumante.

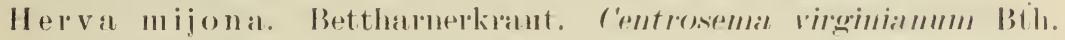
l'apilionalerar.

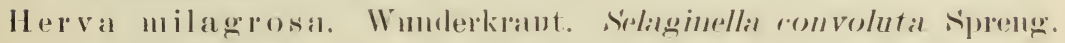
Jyropodiarratr.

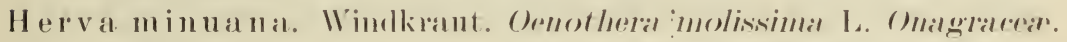

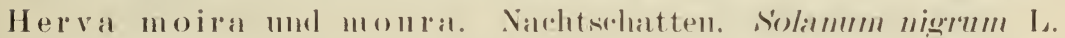
Solillialueile.

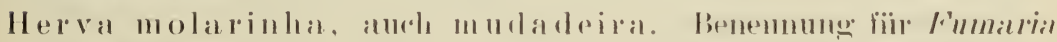
offirinalis L.

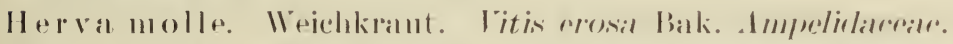

Herva molle falsal. Falsohes 11 . Cimmolurmal mollix Mart. Imat-

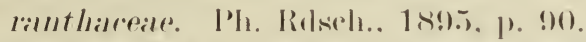

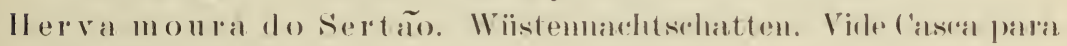
tuilo.

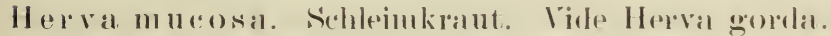

Il erva mular. Manlthierklant. Virle. Mlanmplomeinar.

Herva los mulos. Mallerkrant.

Herva dos na

Herva de Nossa Sienhora. Mutterouteskiat. Virle Cipó de colira.

Herva de pai ('atetano. Vator ('ajetamskraut. Vorlunal littoralis HBKth. Verlemileciar.

Herva de palo. Vide Mate.

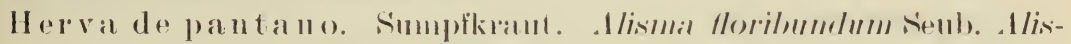

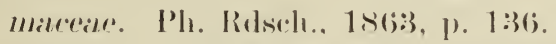

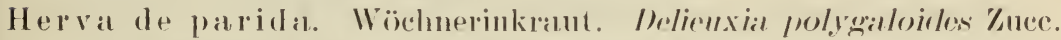

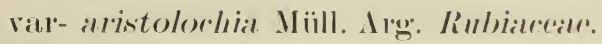

Herva de passarinho. Vogelkmut. Rsittallanthus rolustus Mart. Loraunthalceale.

Herva de passarinlo le abacate. Mbarate V. Plathirusal lorrifolid HBKth. I,orallhthileite.

Herva de passariuho de eacao. ('acan l. Phthirnsa Theobromae Willd. Loranthaceae.

Herra de passarinho de folha larga. Breitblätriges V. Phoradendron crassifolium Pohl. Loranthacear. 
Herva de passarinho de follan minda. Kleinblätr. V. Struthanthus mantrinatus Blum. Lorantharceate.

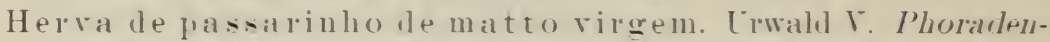
dron latitolinn Griesel. Loranthateap. Arch. d. Pharm.. 5.5. p. 22.27: 10.2. p. 169.

Herva de perdiz. Rebhnhnkraut. Vicle Fruteira de perdiz.

Herva picau. Stechkiaut. Biders pilotus L. Compositar.

Herva le pipi. Vide Raiz de (iume.

Herva de pombinha. Täubchenkraut. Plàllanthus nirniri Müll. Arg. Enpliorluiaceale.

Herva de pombinha hrava. Wilules T. Phrollantluus alcutifolius spreng. Enphorbialceale.

Helva de pombinha miuda. Kleines T. Phrllanthus diffusus Müll. Arg. Euphorlialceale.

Herva de pombinha da serra. Gebirgers. Thallanthus cladotrichlis. Miill. Arg. Euphorlialceale.

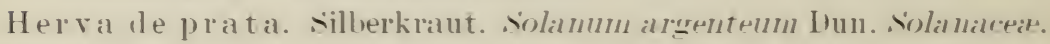
Herva de preya. i Pregakraut. nach dem Meerschweinchen Cavea Herva de préa. i aperea. Fermonial sorploides Pers. Compositar. Herva le pulgera. Flohkiaut. Poligonum hrdropiperoindes Mx.

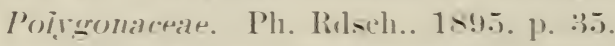

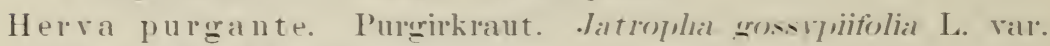

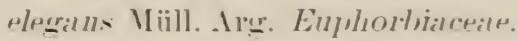

Herra de qua resma. Fastenkiant. Tetranervis Martianal Maud. Melastomialceale.

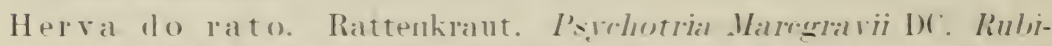

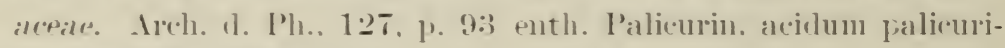
r.um unt Mroetonin.

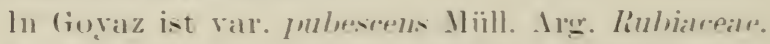

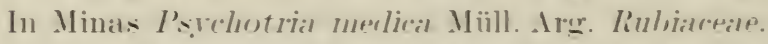

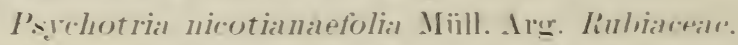

Herva de rat o a malello. Gelbes Rattenkrant. Vide Douradinho

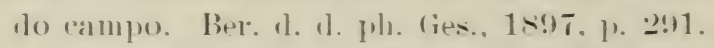

Herva de rato branra. Weisses Rattenkraut. Palicureal oluacal Miill. Iror. Rullialceile.

Herra de ratu hraro. Wildes li. Visle Herva de rato.

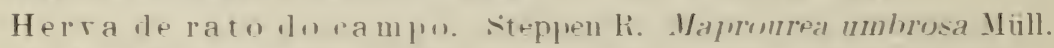

Arg. Rubiarede.

In Bahia Psichotria chloritica var. Bahiensis. Müll. Arg. Rubiacer. 
Herva do rato grande. Grosses R. Prychotria tabarifolia Mïll.

Alg. Rubiacene.

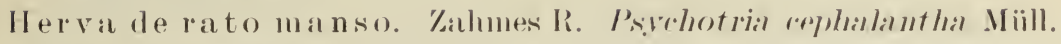

Alog. Rubialerar.

Herva de rato pelluda. 'zottiges R. Vide Herva de rato in Goyaz.

Die siimuntlichen Psychotria-Arten, mit Iusnahme von P'. cephislintha, werden als Rattengift benutzt, sind uur im frischen /ustande toxisch. getrocknet unschällich. Sach Engler und

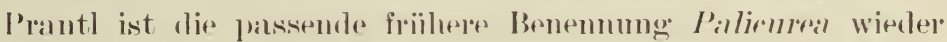
eingefiilnt.

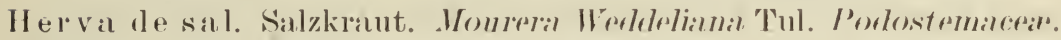

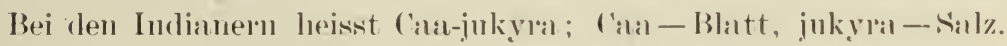

P'ı. Relsch., 18!)4, p. 2) 40 .

Herva sangue. Blutkraut. Vide Lingua de varcai.

Herva santa. Heiliges Kraut. Barrharlis orhrareal Silleng., Barrcharis vulnerartil Bak. C'ompositare.

Herva santa do campo. Steppen Heiligeskiaut. Barroharis Lambii I)(. C'ompositilr.

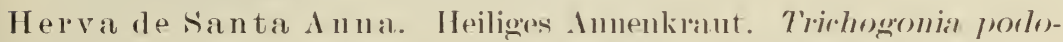
rarpa Schulz-Bi. Compositare.

Helva de śanta Cruz. Heiliges Kreuzkaut. Vernonial ammophila Crard.; Caralial forlunculatil Vellos. ('ompositare.

Herva de Santa Eugenia. Heiliges Eugenienkr. Virle ripo de lavandeira.

Helva de Sianta Helena. Heiliges Helenkr. Stevia salturcifolial

Schulz-Bi. var. hirsuta rompositar. Arolı. d. Plı., 12., p. 115: 102. P. 166.

Herva de šanta Luzia. Heiliges Lncienkr. Enphorbia hrasiliensis

Lam. Euphorbiareae. Heisst auch Caa-tia.

Herva de Sianta Maria. Heiliges Marienkr. C'henopodimu ambrosoides L. var. dentatil Fenzl. Arch. (1. Plı., 8.2, p. 9.; Ph. Ralsch., $189 \%$, р. 89.

In Bahia heisst Mentrusto; in Mlagoas-Panamà; in Maranlano Cravinho do mato und in Para-Herva santa.

Herva des. Caetano. Vile Melao de S. Caetano.

Herva de S. Joa o. Heiliges .Johanniskraut. Ageratum roniroirles

L. Compositae. Enthält Cumarin. Arch. d. Ph., 91, p. 109.

Tupy Camará-apeba. Camptosema erythrimoides Bth. Papilionalrear. 
In Bahia Ageratum melissaefolium DC. Compositae.

In Pernambuco Melampodium ramphoratum Bak. ('ompositae.

In s. Paulo vide Mentrasto.

Herva de s. Joa o do campo. Steppen H. Ikr. Grlechon organifolia Bth. Labiatae.

Herva de S. Martinho. Heiliges Martinskraut. Sauvalgesia erertil

L. Sanvagesiaceae. Ber. d. d. ph. Ges., 1897, p. 288.

Herva de s.' Panlo. Heilig. S. Paulskrant. Benennung fiir Mate. Herva de s. Pedro. Heilig. Peterskrant. Hrytis rerluenarolial

Mart. Labiatile.

Herva de sapo. Froschkraut. Bogonial hidentatal Rarll. Begoniaceae. Ph. Arch., 1899, p. 99.

Mikania rordifolia Willd. var. eormulosia Bak. Compositue.

Herva de saracíra. Wasserhuhnkmut. Begonia hirtella Link.

Begoniacease. P'l. Arch., 1899, p. 92.

Herva de Sassafraz. Rnilgeal sulsessilis Bth. Rubiaceale.

Herva de sereno. Taukrant. Conyzal Chilensis Spreng. Compositir. Herva Silvina. Polvporlinm lyroporlioirles L. I'olvporliareae.

Herva de soldado. Soldatenkiaut. Artanthe elongata Miq. Pi-

peraceas. Pls. Ridscli, 1894, p. 242.

In S. Painlo vide Ambar vegetal. Plı. lidsch., 1894, p. 240.

Herva terrestre. Vide Figneira terrestre. Ph. Rdsch., 1891, p. 291. Herva tostao. Borrhal ria hirsuta Willd.; Borrhavial paniculata

Rich. Nyetaginareale. Arch. d. I'ham., 12:3, p. 11.; Plı. Review, 1896, pp. 82 and $8: 3$.

In Minas Boerlhavia erecta I. Nyrtaginareale.

Herva de touro. Stierkraut. Vide Herva de S. Martinlo.

Herva ullicina. Conton arlipatus Ktlı. Emphorldiareae.

Herva de vida. Lebenskrant. Heimia salicifolia Link. Lythraceas. Herva de vintem. P'ennigskiant. Toyria nniffora Lam. Cien-

tianareane. C'urtia tenuifolia Knoll. Ber. d. d. ph. (ies.. 1899, p.Herva de veado. Rehkraut. Jonidinm higihlossum st. Hil. Vio-

likeas. Rer. d. 1. ph. (ies., 1897, p. 104.

Herva venenosa. Giftkrant. IMiplanlenia ilhustris Müll. Arg. Apocynaceae.

Herva de vidro. Glaspflanze. Vide Lingua de sapo.

Herva viva. Vide Sensitiva.

Herval. Ein Wald von Matehïmmen.

Hervateiro. Matehändler. 


\section{7}

Hervinha de parial. Wöchnevinnenthee. Iechenxial pollgaloirles \%uec. Rubiaceale.

Hervinha secea. Trocknes Kräutchen. Parmelia rocellit L. Lichenes.

Heymassoli. Vide Imbuim.

Hiapam und Hiapim. Tupyname fïr Herva Santa.

Himilao. Indianername fïr Pichurimbohne.

Hiva-hai. Vicle Ahomay.

Hortelaa pimenta. Menthal piperita L. Lallialtar.

Hortelaa sylvestre. Wilele Mïnze. Viele Mentrasto.

Huatea. Vide Guacao.

Huacava. Virle Anají. Ph. Rdsch., 1889, p. 1:34.

Hua imy. Virle Vami. Ph. Relseh., 1888, p. 264.

H uaichich. Benennung ler ('hiquito-Indianer fiir sialo.

Humary. Humirial florilumblil Mast. Valr. montanil Urh. Mumiriareare. In Parai eine essbale IValdifrucht (unbestimmt).

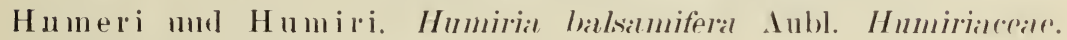
Ber. d. (1. ph. (ies., 1s!)!, p. $4+$.

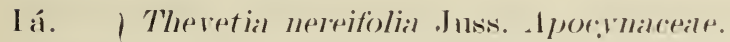

Iáca.j Heisst auch Igualiy.

Ibabiraba. Britoal trifloral Bğ. Mortalceale. Tupy ibá-Holz. beráb-Selhlenter.

Iba-cam uci. Bauholz in Permamburo.

Ila-cocaba. Vide Acapociba.

Iba-curu-pari. Ville Bacopari.

Iba-guassu. Tupiname fïr Melancia.

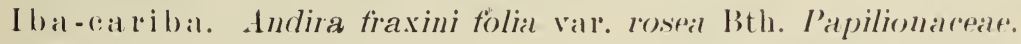

Iba-metara. Vide Acaya. Ber. d. 1. ph. (iess. 18!s. 1). 15:3.

Ila-pitanga. Vide Pitangeira.

I ba-pocaba. Vide Acapociba.

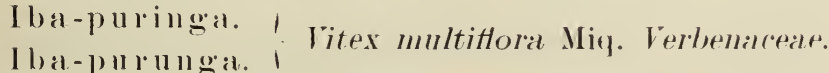

I ba-pyta. Coros alustralis Mast. Palmare.

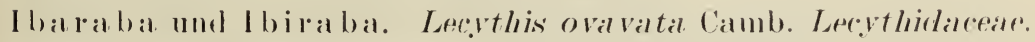

I bara ent. Periandra roceineal Bth. Papilionaceate.

I baremo. Vile Cipo alho.

Ibaró. Vide truxto de sabaio.

Ibiacariba. Vicle Iba-iariba. 
Lbira. Mylopia hrasiliensis Spreng. Anonaceae. Ber. d. d. ph. (ies., 1897. p. 464. Heisst auch Conquerecou.

Ibira-ce. Vide Buranhem.

Ibira-obi. Vide Paó ferro.

Ibira-paye. Vide Balsamo.

Ibira-pitanga.

Ibiri-pitang-assú. I Vide Paó Brasil.

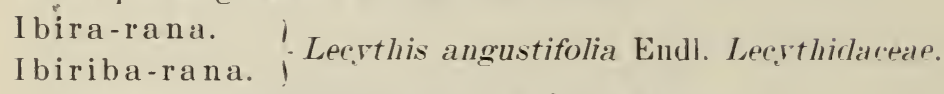

Ibiriba. Lecythis Luschnatii Bg. Lec.thidaceate.

Ibiruba. Vide Pitangueira do mato.

Ibirubé. Vide Jaracathia.

Ibixuma. Vide Mutamba.

I byra und Ibyra-quiynha. Vide Craveiro de Maranhaó.

I c̀̀. Glasiora Martiana Dr. Palmae.

I cà-assú. Glasiova insignis Dr. Palmae.

I с ас o. Vide Goajerú.

Icacoré catinga. Ardisia semicrenata Mast. Var. repandal Miq. My winaceate.

Icicariba. Vide Almessegueira. Ber. d. d. ph. Ges., 1898, p. 441. I cipó. Tetracera oblongata DC. Dilleniaceac.

Iciy. Vide Almessegueira.

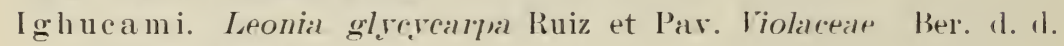
ph. Ges., 1897 , p. 104.

$1 \mathrm{mb}$ a im. Vide Ameixeira de espinho.

I m ba met al l’á und I mbemeta rá. Vide Acara. Ber. d. d. ph. Ges., 1898. p. 15:3.

I mbauba. Vide Ambaíba. Ph. Rdsch., 1891. p. 290: Alch. d. Ph.. 1857. Bd. 89 . p. 247 .

Im bé. Vide Cipo imbé. Ph. Rdsch.. 1892. p. 281.

I m bé de comer. Essbare I. Philodendron guttiferum Btlı. dractrate.

Ph. Rdsch., 1×92, pp. 279-281.

I m bé manso. Zahmer I. Monstera pertusal D. Alacear. Plı. Risch.. 1892, pp. $279-281$.

I $\mathrm{mb}$ be $\mathrm{miu}$ ilo. Kileiner I. Phiiodendron ochostemon sichott. Araceit.

Pl. Rdsch., 1\$92, pp. 279-281.

I $m$ bé de priaia. Küsten I. Montrichardia arborescens schott.

Araceae. Ph. Rdsch., 1892, pp. 279-281.

$1 \mathrm{mbé}$ des. S. Pedro und Imbé-rana. Vide Dragao fedorento. 
I mberi. Vide Panduarana.

I mberi cipo. Diorlea violacea Mart. et Bth. Papilionaceae.

I mbérova. Aspidosperma parvifolium A. DC. Aporynaceas.

Imbira. Vide Pindahyba. Arch. d. Ph., 1862, Bd. 110, p. 1:37.

Imbir-areia. Vide Mata paó de espinha.

Imbira de seda. Aranjia subhastata Fourn. Asclepiadacear.

Imbir-assí. Bombax marginatum Schum. Bombareage Bombax hexaphyllum. Vellos. Bombarreale.

In Minas Bombax tomentosum St. Hil. Bombarrate.

In S. Paulo Bombix pubescens Mart. et Luce. Bombaceale.

I mbir-assí de folha lisa. Bombax gracilipes Schum. Bombaceae.

Imbir-assí do mato. Bombax longiflorum Schum. Bombaceate.

Imbir-assú mata paó. C'horisial Riveri Schum. Bombaceale.

Imbira-tanha. Guazuma crinita Nart. 'Stermliaceap.
Eriotheca pubescens Hend. is

Imbiri. Esterhazia splendida Mik. S'rrophulariaceate.

Imbui. Vide Embyí.

Imbí. Vide Acaya.

I m burana. Bursera leptophloes Engl. Burseraceatr. Ber. d. આ. ph. Ges., 1898, p. 438.

I m burana do Campo. Bursera Martiana Engl. Burserarease.

Imburana macho. Männlicher I. Amburana Clandii Schw. et

Taub. Papilionaceas. Samen sehr reich an Cumarin.

I m buri. Diplotheminm candescens Mart. Palmae. P'h. Rdsch., 1889,

p. 110.

I mburi do cachorro. Vide Coco de cachorro.

I mbury. Vide Palha branca.

Imbuzada. Getränk aus dem Safte der Aravafrucht mit rohem

Rohrzucker und geronnener Milch.

Imbuzeiro. Vide Acaya.

Imbyra-tanha. Vide Imbira-tanha.

Immortal. Vide Córal.

I myra-quiynha. Vide Craveiro da terra.

Iñagerana. Bysonima spicata Rich. Malpighiacear.

Inajá. Maximiliana regia Mart. Palmae. Ph. Rusch., 1889. p. 134.

Inajá-guassú-iba. Tupiname für Cocos nucifera L.

Inajá-y. Vide Pupunha de porco.

Inambú-quissana. Bauholz in Amazonas und auch Inameihy. 
Incenc;o. Weihrauch. Protinm multiflornm Burserareas. Ber. d. d. ph. Gies., 1898, p. 440.

Incenço. Pittospermum coriacenm Ail. Pittosporaceae. Eingeführt. Inci. Virle Icó.

Indaya. Harte Frucht (ron ainda-hart, ya-Frucht). Attalea Indi.la I)r. Palmae. Ph. Rilsch., 1889, p. 112.

Indlä-asíl. (irosise. Attalea rompta Mart.; Orbignea macro(arpal Barb. Rodr. Palmar. Ph. Rdsch., 1889, p. 112.

In d a ya do cam po. Steppen. Attalea Cearensis Barb. Rodr. Palmar. Ph. Rdsch., 18х9, p. 112.

Indaya mirim. Kleine. C'oros longebracteata Barb. Rodr. Palmir. Ph. Rrlsch., 1889. p. 112.

Indaya redonda. Runde. Coros campestris Barb. Rodr. Palmap. Ph. Relsch.. 18x!), p. 112.

Indigo sylvestris. Wilder Indigo. Solanum indigofera St. Hil. Solanareite.

Infallivel. Enfehlbar. Gomphrena Pohlii Miq. Amarantaceap. Ph. Ralsch.. 189.), p. 90. Piptorarplia rotumrlifolia Bak. C'omjositar.

Ingá. Inga affinis I)( Minnosareate. Arch. d. Ph., 91, p. 241.

I nga a a marella. Gelbe I. Inga fagifolia Willd. var. perlicellaris I3th.

Mimosaceae. lonnbretun Lopffingii Eichl. Combretareas.

Inğ a margosa. Bittere I. Sirartzia elegans sichott. C'apsallinfacear.

Ingá assú und I. ẹuassí. Grosse I. Ingar rinnamomea śprue. Minnosicpile.

Ing̣a boi. Ochsen I. Swartzial Flamengii Radd. C'acsalpineareate. Ingá hol da da. Gesänute I. Vide Ingá minda.

Inga branco. Weisse I. Inga allo Willd. Mimosaceae.

Inga bra ro. Wilder I. C'alliandra Perkoltii Bth. Minnosaceate. I ngá cabelluda. Hailloges I. Virle Ingá.

In Ingai Cangallıa. Lastrattelholz. Inga Thibandiana I)C. Mimosarear. Inga chichi und xisci. Inga sertulifera Dr. Minnosaceate.

Ingá chichi branco. Vide Inga branca.

Ingá сіро́. I Lianell I. Ingal elulis Mart. Mimosalcear.

Ingá doce. i sïisser I. 
Ingá doce da serra. Inga hiarbata Bth. Mimosalcaile.

Ingá fava. Bohnen I. Inga faral Willd. Mimosalleale.

Inga fava-hy. Kleine Bohnen I. Ingal ror listipmlil Malt. Mimosilceae.

I no á do foga. Ingá ——

Ingá do fogó-hy. Viøle Ingá fava-hy.

Ingá mimosa. Karte I. Ingal filgifolial Willıl. Mimosiarease.

Ingá mirim. Kleine I. Ingar rolindriral Mart. Mimosiareate.

Ingá miuda. Ingan maroinata Willd.: Calleandral reanrensis Taub.

\section{Mimosalceale.}

Ingá opeapiiba. Inga maritima Bth. Mimosaceale.

Ingá de quatro follas. Vierblätrige I. Vide lngá amarella.

Ingá de rio. Víle Ingá mimosa.

Ingai vermelha. Rothe I. Ingar Mnleis Mart. var. Jarvifolia Bth.

Mimosareale.

In haiba. Vide Canella preta.

Inhame. Colorasia antiquormm Sch. Iraceate. Pls. Rdsch., 189:3.

Inhame branco. Colorasial antiquornm valr. trpica siols. Aralreare.

Ph. Rdsch., 189:3.

In ha me de ('ariola. Vide Carí de Angola.

In lı me da costa. Afrikanische I. Vile Cára de (ininea.

In li me cigarra. Dioscorea Lalrerdilei Grieseb. Mioseorearreale. Ph.

Risch., 1894, p. 189 .

Inhame nambì Inosroreal trifoliata Kth. Iioscoreareate. Ph.

Rdsch., 1894, p. 189.

In hame roxo. Rothbrame I. Rajania hastata Lam. Liliarear.

Inhame rermelho. Lothe I. Colocasia antiquornm Sich. var. areris.

Arareate.

In ha pé-canga. Virle Japecanga.

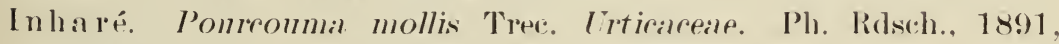

p. 289.

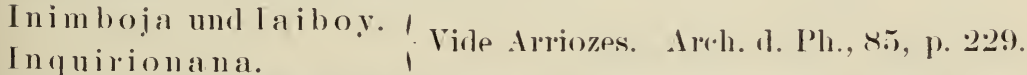

Inquiri. Vide Malicia de mulher.

I pa ca muci. Vide Jaracathia do mato.

I pa dì. Erythroxylom rora Lam. Erythroxylaceap.

Ipad ì mirim. Kleine cora. Erothroxylum catarartornm Spruc.

Erythroxvlatceile.

I pé. Tecoma ipé Mart. Bignoniareale. 
Ipé a marello. (ielbe 1. Teroma orhrareal ('ham. Bignoniarease. Arch. d. Ph.. 91, p. 240.

I pé assín. Vide Ipé tabaco.

I pè batata. Knollen I. Tecoma tuherculosa Bur. Bignoniaceae. Ipé boia. Vide Mossetaiba.

I pé braneo. Weisse 1. Patagonula rulneraria L. var. hirsuta Tria.

Cordiaceate.

In Bahia Patagonula bahiensis Moric. Cordiaceae.

In Minas Teroma lorgiflora Bur. et K. Schum. Bignoniaceale.

In Rio Grande do sul Patagonulit americana L. var. hirsuta Tra. Cordiaceae.

In Rio de Janeiro Cassia micranthera DC. Caesalpineaceae.

I pé do campo. Steppen I. Vide Ipé ipeura.

I pé contra sarnas. Krätzeheil I. Tecoma impetiginosa Mart.

I pé de flor roxa. Violetrothe I. Sparattosperma nenroralix Bur.

et K. Sich.

I pé ipeura. Teroma araliacea P. DC.

I pé mirim. Keleine I. Tecoma splendida spr.

Ipé roxa. Vide Ipé contra sarnas.

Ipé tabaco. Tabaks I. Tecoma chrisotricha Mart.: Teromal peclicellata Bur. et $\mathrm{K}$. sich.

I pé tinga. Weisse I. Vide Ipé braneo in Minas.

Ipé varzea. Thal I. Vide Ipé ipeura.

Ipé verde. Griine Ipé. Vide Caroba de flor verite.

Ípé-caa-goene. Tupybenennung für Iperarluanlial.

I pecacuanha.

I pecacuanha branca.

Ipecacuanha branca.
Ipecacuanha da praia. Side Poaya.
Ipecacuanha preta.

I pecacuanha falsa. Euphorbia Ipecaruanha V. Enphorliaceate.

I реи́ra. Vicle ('inco folhas. Areh. 1. Pharm.. 32. pp. 166; d 239: 102 , p. 166.

I pobacaba. Vide Acapociba.

I p̀̀. Vide Batata de purga. Areh. d. Ph., 18.52, p. 316: 18.55, p. 93. Iraca. Vide Jijiịapa.

Iraiba. Vide Paty amargoso.

Iririba. Vide Arariba.

Irucurana. Vide Brejauba. Ph. Rdsch., 1888, p. 262.

Isca do mato. Waldzunder. Botritis fomentaria Mart. Hrphomycetes. Areh. d. Ph.. 110, p. 134. 
Issicarloiba. Vide Icicariba.

Issitantan. (Tupy Yeica - Har\%, antan - klebend.) Protium pubescens Engl. Burseraceate. Ber. d. 1. ph. (ies., 189\%. p. 440.

I tabì. Vide Itaubì.

Itaborana. Bauholz in Innazonas.

I tam $\mathrm{um}$ beca. Ditto.

I tap icurú amarllo. Bauholz in Magoas. Forhysiarear.

I tapicurí. Vide Guarubú.

It apiúna. Callisthene maior Mart. Foch.ssiacear.

Itatiba. Bauholz in Parà.

I taйba. Acrodiclirlium Itaubar Meissn. Laurarear. Ph. Rev., 1897: Ph. Rdsch., 1896.

I taíba amarella. Gelbe I. Var. amarello Meissm. Lantrarear.

Ph. Rev., 1897; Ph. Rdsch., 1896.

I táb ú branca. Acrodiclidinm guyanensis Nees. var. ran filtum

Meissn.; Acrodiclirlinm oppositifolinm Nees. Lannaceate. Ph. Rev., 1897 ; Ph. Rdsch., 1896.

I taи́ba peninga. I Rothe I. Acrodiclidinm anacardioides siprux. I taíba piranga. i Lanraceate. Ph. Rev., 1897; Ph. Rdsch., 1896.

Heisst auch Cedro pardo.

I t á ba preta. Vide Cedro cinzento.

I taúba vermellıa. Vide Itaúba pirangáa.

I ta ubú. | Noisettia longifolia HBKth. Violacear.

I taubú amarello. i Ber. d. d. ph. (ies., 1897, p. 101.

I tú. Vide Paò ferro.

Itúa. Gnetum nodiflorum Ad. Brog. Gnetaceate.

I tu bú. Hybanthus ipecacuanha Taub. Violaceae. Ber. d. d. ph. (ies., 1897 , p. 100 .

I vatinga. )

Ivantinga. Tide Azoito carallo branco. Ber. d. d. ph. Ges., I vitinga. 1897 , p. 28.5 .

I va-u mbu. Bamm in den Nordstaaten, essbare samen.

J aborandi. Ein rom Volke adoptirtes Tupiwort, welehes vielen schweisstreibenden Gewächsen beigelegt wird, als: Artanthe Lusnathiana Miq. Artanthe geniculata Miq. Enkia cyanothifolia Kth.

Piperaceae.

Pilocarpus pinnatifolius Lam. Rutaceae. in Pernambuco: 
Zanthoxylum elegans Engl. Rutacede. Ber. d. 1. ph. Ges., 1899, p. 327. Vide Alfavaca hrava und $\mathrm{A}$. de cobra.

Vide Jaguarandi und Jaurandi.

Jaborandi bravo oder do mato. Wilde J. Ottonia anisum

Spreng. Piperaceae. Ph. Rdsch., 1894.

Jaborandi do brejo. Sumpi J. Pilocarpus giganteus Eudl. Ru-

taceae. Ber. 1. d. ph. Ges., 1899, p. 327.

Iaborandi cepoty. Darmwurzel J. Artanthe cernua Presl. I'iperaceale. Ph. R Isch., 1894.

Jaborandi manso. Zahme J. Artanthe mellicoma Miq.; Ottonia proquinqua Kth. Piperaceae. Ph. Rdsch., 1894.

J a botá. (Von Tupi jaboty-Bindepflanze.) Anisosperma passiffor:a Mans. C'ucurbitaceae.

Jabotá pita. (Von jaboty-binden, pita-Bast.) Ouratea Jabota pita Engl.; Ouratea hexasperma Baill. var. Planchonii Engl. Ochnaceae.

Jabotá do campo. Steppen J. Ourateal hexasperma Baill. forma typica Engl. Ochnaceae.

Jabotá cheirosa. Wohlriechenle J. Ouratea suaveolens Fingl. Ochnaceale.

Jabotá mirim. Kleine J. Onratea parviflora Bail. Ochnaceate.

Jaboticaba. Frucht ron Jaboticabeira.

Jaboticabeira. (Von jabotim-Schildkrötensamen, da die Frïchte an der Rinde des stammes befindlich.

In Minas Myrciaria cauliflora Bg. Myrtaceae. Areh. d. Pharm., 123, p. 115 .

In Rio de Janeiro Myrciaria Jaboticaba Bg. Myrtaceae.

Jaboticabeira branca. Weisse J. Myrciaria tenella Bg.; Myrciaria pallida Bg.; Gormidesia reticulata Bg. Myrtaceae.

Jaboticabeira de bugle. Indianer J. Vide Buranhem.

Jaboticabeira cabelluda. Haariger .J. Myrciaria delicatula Bg.

Myrtaceile.

Jaboticabeira de cabinho. Vide J. in Rio de Janeiro.

Jaboticabeira de campina. Vide J. in Minas.

Jaboticabeira de mato. Wald J. Myrciaria äistichal var. Bathiensis Bg. Myrtaceae.

Jaboticabeira miuda. Kleine J. Myrciaria trunciflora Bg. Myrtaceae.

Ja bo ticabeira pelluda. Vide J. cabelluda. 


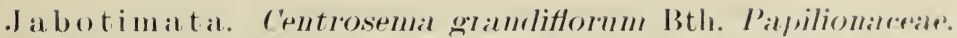

Jaboti membecal. I Karte.J. Piperomia pellurirlal H. B.Kth. PipertJaboti memberi. i aceare Ph. Rolscis., 1894, 1\%. 240.

Jaboty. Erisma calraratum IVarm. Jorlyssiarear.

Jabuticaba und Jabuticabeira. Vide Jaboticabeira.

J a ca. Artocarpus integrifolial I. Irtiralreate. Aroh. 1. Ph. 102. p. 16;

Plı. Rdsch., 18!)1, 1) 221.

Jaca dos pobres. Brodfucht der Armen. Vide Biribá, heisst arkelt

Bibibá-1’ana. Ber. (1. 1. ph. Ges., 1897, p. 4ti2.

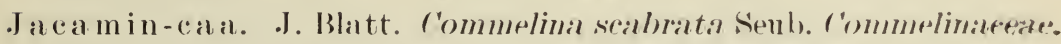

l'h. Rolsch., 1892. 1. 2..s8.

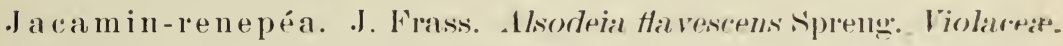

Ber. 1. 1. plı. Ges., 1897, 1) 104.

Jac:anine-cal. Vide Marianinha.

J acapé. Vide ('apim cheiroso. Ph. Rokch., 1894, p. 1הт.

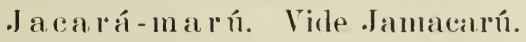

Jacalandá. (Von Ya und (araná.) Marlaxerinm legale Bth. V'apilionaceate.

Jacaranda antan. Fester .J. Marharinm lamatum Ful. Pari-

lionaceare.

J acalanda banana. Bananen .J. zufolge der gellen bananenihn-

lichen Schote. Swartzia Langsslorffii Radd. Mimosialreas.

Jacarandá branca. Weisse. J. Platiporlinm elegans Toug. rar. maior Bth. Palpilionareare.

J a carandá Cabiúna. Dunkle .J. Marhaerium incorruptibole Fr.

All.; Dalbergia miscololium Bth. Papilionaceate.

J a car andá de c a mpina. J. der Ebene. Swartzia gramtifolia Bong:-

Mimosaceae.

Jacarandá do campo. Nteppen .J. Hecisstophrllum monetaria

Peis. Papilionaceale. Ville auch Jacarandá antan.

Jacaraudá de espinho. Dorniger .J. Mardharinm lencoptertrrs

Vog. Papilionacene.

Jacarandá macho. Männlicher J. Vide Jacarandá branca.

Jacarandá pacova. Grosser Bananen .J. Suartzia Flemmingiz

Radd. Mimosacene.

Jacarandá parda. Bramuer J. Marhaerinm rillosmm Vog. Mazpilionareae. 
Jacarandá pirangạ. Rother J. Vide Jacarandá rosa.

Jacarandá preto und J. pixuna. Schwarzer J. Dalbergial nigrä

Fr. All. Papilionacear. Polisander. Arch. d. Ph., 93, p. 119. Jacarandá preta in S. Paulo. Vide Carobinha do campo.

J acarandá pui-tan. Vide Jacaranda tan.

Jaca ra ndá rosa. Rosen J. Drenocarpus microphrllus Wawra. P’a-

pilionaceae. Wohlriechendes Möbelholz, liefert 0.37 Proc. aeth. Oel.

J acarandá roxa. Violetrothes .J. Macharerium firmmn Bth. P’apilionaceae.

Jacalrandá dos taboleiros. Hochebenen J. Marhaprium murronulatum Mart. Papilionacear.

Jacarandá-tan. Hartes J. Marhaerium Allemanni 13th. Palpi-

lionaceae.

J a ca ratéa. Indianische Benennung für vide Mamoeira.

Jacará-tinga. Weisser Eidechsenbaum. Engenia inorarpal I)(.

Mirtaceae.

Jacaré. Kaimanbaum. Vide Ifongoló.

Jaca ré-arń. Grosser Eidechsenbaum. Vide C'aferana. Ber. 1. 1. ph. Ges., 1899, p. 226.

J a ca ré-ca tinga. Stinkender Kaimansbaum. Anlon!rcla leuradendron Bg. Myrtacease.

J ac a ré-opaiba.

Jacaré-huiba.

Jacaré-uba und yba. I Ber. d. d. ph. Ges., 1897, p. 238.

Jaca ré-cui-taua. ) (Von Jacré-Kaiman, cui-Beere, taua-gelb. Jacaréy-ataua. I Reisserckia rordifolia Steud. Rhamnaceae.

Jaca ré-mirim. Vide Itipiúna.

J a catirao. Miconia thearams ('ogn. Melistomaceale.

J a c a tupé. Vide Jacutupi.

Јасє. Guaraniname für Melancia.

Jacitarì. Vide Titarà.

Jacúa. Bauholz in Alagoas.

Jacúa-cangà. Vide Aguarà-cuinha.

Jacúba. Erfrischendes Getränk aut Reisen, von geröstetem Matismehl (rapadura) und Wasser.

Jacunda. Calathea ornata Rche. var. lineata Hub. Marantareate. Jarutupé. Parhyrrhizus. angulatus var. integrifolia Bth. Papilionarpate. 
Jaca pucaya. Tupiname. Vile Sapucaia.

Ialao und Jambelao und Jamelao. Sizyginm .Jambolanum I)(.

Myrtacease.

J ago irana. Virle Juguarana.

J aguarandi. Pilocarpus Jaboramdi Holm. und vile Jaborandi do brejo. Rutaceae.

Jala pa. Ipomoea purga Wendr. Convolvulareate.

Jalapa do Matto Grosso. Calvstegia palmato-pinnatil Meissn. Convolvulaceate.

In Minas Ipomoea punicea Chois. Convolvulacear.

In S. Paulo Ipomoea echioides Chois. Convolvulaceate.

Jalapa branca. Weisse .J. Macrosiphonia longifloral Miill. Arg.;

Diplodenia illustris val. Rubescens Miill. Arg. Apocinaceae.

Ja lapa do campo. Steppen.J. Marrosiphonia punicea Chois. Var. rariftora Meissn. Apocinareate.

Jalapat eomprida. Lange J. I Mirabilis .Jalallal L. Nyctaginalcer. $\begin{array}{ll}\text { Jalapa domato. } & \text { Wald J. j } \\ \text { Jalapa nabic口. } & \text { Riiben J. Ph. Rer., 189(;. 1). } 37 .\end{array}$

Jalapa vermelha. Rothe .J. Dipladenia illustrus var. hirsutil

Miill. Arg. Apocinaceate.

Ja la pao. Grosse .J. Ipomoea jalapal Pursh. C'onvolvulaceate.

Ja lapinha. Kleine J. Ipomoea utilis Chois. Convolvulaceate.

Dipladenia spigeliaeflora Miill. Arg. Apocvnaceate.

Jalapinha comprida. Kleine lange .J. Diplarlenia ntroviolarear

Miill. Arg. Apocvnaceat.

J a ma car r. Cereus .Jamacaru Salzm. Cactaceate.

J a m a car rí do sertao. Cereus geometrizans Mart. Ciactureale.

J a m a rá -caír. Vide Mandacarú.

Ja marú. Benennung der Pflanze und des aus der Fruchtschale bereiteten Trinkgefässes von Lagenaria idolatrica Ser. Cucurbitaceate. Jambó. ，Jambosa vulgaris D(: Mvrtaceae. GewöhnJambó a marello. j licher und gelber $J$.

J a mbó branco. Weisser. J. Jambosa sammangensis I)(. MIrtaceae.

J a m bó de bugre. Indianer.J. Tavomita lencantha Planch. et Triar.

Cinttiferae. Ber. d. 1. ph. Ges., 1897, p. 242.

J a mbó encarna 
Ja mbó da India. Jambosa domestica Rumph. Myrtaceae.

J a m bó vermelho. Rother J. Jambosa malacensis DC. Myrtaceae.

Sämmtlich nur kultivirt in Brasilien.

J a m b ú. Vide Agriao de Parà.

J a mbú-assí. Grosser J.

J a m burana. Falscher J. Vide Imburana.

J andiroba. Vide Andiroba.

J anapuca. Vide Puça.

J a na úba. Melastomaceare. Heilmittel.

Jangada brava. Wildes Flossbontholz. Heliocarpus annericanus

L. var. typica Schum. Tiliacear. Ber. d. d. ph. Ges., 1898, p. 283.

J anga deira. Flossenbootholz. Apeiba Tibourbon Aubl. Tiliaceate.

Ber. d. d. ph. Ges., 1898, p. 2x:3.

J ango. Linum selaginoides Lam. Linaceae. Ber. d. d. ph. Ges., 1899 , p. 45 .

J a niburana. Cotula coronopifolia L. Compositae.

Janipabo und Janipapo. Vide Genipabeira.

J a nip a r a n di ba, Janiparanduba, Jandiparana. Vide Japaranduba. Janua-uva. Plumeria attenuata Bth. Apocynaceae.

Japa. Indianische Benennung der Matte ron geflochtenen Palmblättern.

Ja pa-camin. Securidaca ? Scrophulariaceae.

Japarandiba und Japorandiba und Japoaranduba. Gustavia

brasiliana DC. Myrtaceae.

J a pecanga, Joapitanga, Jupicanga. ('Tupi von Jepo-canong-sich

heilen.) Sinilax japicanga Grieseb. Smilaceae. Arch. d. Ph., 123,

p. $115 ; 103$, p. 38; Ph. Rdsch., 1893, p. 82.

Japecanga miuda. Smilax brasiliensis Spreng.

J a pecanga em S. Paulo. Vide Arrebenta.

J a qua. Lucuma gigantea Fr. Allem. Sapotaceae.

J a queira. Vide Jaca.

Jará. Mauritiana Martiana Spruc. Palmae. Vide Mahi.

J a ŕá assí. Grosse J. Leopoldina maior Wallace. Palmae.

Jar ácathia. Cereus melamerus K. Schum. Cactaceae.

J á ra cá tia. Jaracatia dodecaph.lla A. DC. Caricaceae. Arch. d. Ph., 91, p. 111. 


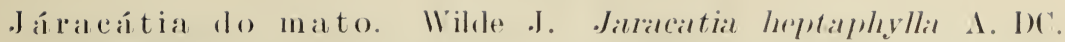
('arialeale.

Jarámacarú. Vide Mandacarú.

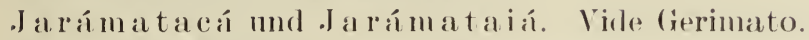

J a raná. Schiffsbauholz in P’àì.

Ja raudina. Bauhol\% in P'arà.

Jararacá und .Jararaca-taia. Dracontinm aspermm ('. Koch. Araceate. Arch. d. P'ı, 8.5, 1). 228.

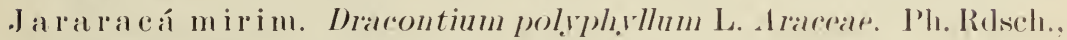
$1892,1 \% .280$.

Jararacá miudo. Kéleine .J. Stanrostigmal Lnschnathianum ('.

Koch. Araceate. P'h. RAlsch., 18!)2, p. 280.

J a rarana. Gooros aequatorialis Barb. Rodr. I'almane.

Jarbac. Vide Aguará-pondé.

Jarere. Vide Amendoim. T'upiname.

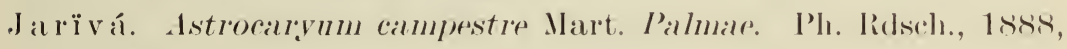
p. 261 .

Ja roba. Solannm C'arolinense L. Solinnareale.

Jarinha. Kleine Krugpflanze. Bristolochia Cilasiovii Mart. - 1. macromia Gomes.

In Bahia Aristolorlhia tribolata $\mathrm{L}_{\text {.; }}$;

In s. Paulo ) Aristolochial Burchelli Mart.-A. (hilmissonis Duch.

11. in Minas i 1. brasiliensis Mart. var. parvifloral Duch.-1. (renatil Mart. Aristolochiaceat: Ph. Rdsch., 189:3, p. 181.

Jarrinlı batatinha. Kileine Knollenkrugpflanze. Aristolochia

filipendula Inch. Aristolochiaceite. Ph. Rdsch., 189:3, p. 181.

Jarinha do campo. Steppen K. Aristolochia Clausseni Inch.

Aristolochiaceale. Ph. Rdsch., 1893, p. 181.

Jarinha cheirosa. Wohlriechende K. Iristolochia odora steud.

Aristolochiaceate. Plı. Rasch., 189:3, p. 181.

Jarrinha miuda. Kleine K. Aristolochia ramicifolia Steud. Aristolochiaceate. Plı. Rdsch., 1893, p. 181.

Jarro. Krug. Vide Mil homem.

Jarro de capacete. Helmkrugpfl. Aristolochia galeata Mart. et

Zuce. Aristolorhiaceare. Ph. Rolsch., 1893, p. 181.

Jarro manchado. Gefleckter Krug. Vide Járaracá.

Jaruma. Ville Imbaitinga. Ph. Rolsch., 1891, p. 289.

Jasmin bravo. Wilder Jasmin. Tabermaluontana rubro-striclatal

Mart. Apocinareale. 
Jasmin da cerca. Hecken .J. Jasminum azoricum L. Jasminaceat. Kultivirt.

Jasmin galego. P’ortugiesischer .J. Jismminnm offirinale L. Jasminareate. Kultivirt.

Jasmin da Italia. Italienischer .J. Jasminnm angustifolinm IV. Jasminilceale. Kultivirt.

Jasmin larania. Orangen .J. Mnrralal exotical L. Rutareate. Kultivirt.

Jasmin de leite. Milch .J. Aranpa hortornm Fourn. Asclepiarlacear. Jasm in m a ngí. Plumeria rubra L. Apocrnaceat. Verwildert.

Jasmin do mato. Wald J. Basanacantha spinosa var. pulsescens Schum. Rubiaceate. Arch. d. Ph., 110, p. 135.

In Rio Grande do Sul. Guettarlar nrugnensis Cham. Rubiateale. Jasmin do reino. Vide Jasmin da Italia.

Jasm in de S. José. Plnmeria loranthifolia Mïll. Arg. Apocynarear. Jasmin de soldado. Soldaten .J. Vide Cinnamomo.

Jasmin dos taboleiros. Hochebenen J. Spriranthera odoratissimal st. Hil. Rubiaceae.

Jassahi. Vide Jatoba.

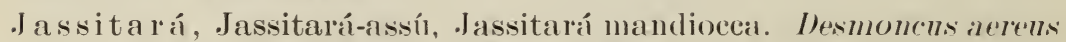
Dr. Palmae. Ph. Rdsch., 1888, p. 26.5.

Jassitará merim. Kleine J. Desmoncus mitis Mart. Palmite. Ph. Rdsch., 1888, p. 265.

J a ss ita rá-pui. Desmoncus pumilus Trl. Palmae. Plı. Rolsch., 1888, p. 26.5 .

Jassitará-tiputi. Desmoncus macrocanthus Mart. Palmae. Ph. Rdsch.. 1888, p. 265 .

Jatá. Cocus Sragrus Dr. Palmae. Plı. Ridsch., 189:3, p. 38. J at aboca. Arundinaceae in Pernambuco.

Jataiba. Virle Tatagiba.

J a ta ua-rana. Hymenaea splendida Vog. Citesalpineaceate.

Jataúba und Jata-úva. Vide Jatí. (úva= Baum.)

Jatay, Jatahy. Vide Jetahy.

.J a tay-mirim. Kleine .J. Vochysia ferruginea Mart. Vochysiacear. Heisst auch Data mirim.

Jatitará. Desmoncus polyacanthus Mart. Palmae. Ph. Rdsch., 1888.

Jatobá, Jatuba. Hymenaea stillocarpa Hayn. Caesalpineaceae. Ztschr. d. a. ister. Apoth.-Ver., 1868; Arch. d. Ph., 82, p. 95. 
Ja toba do campo. Steppen .J. H.menapa rhaparlensis Barb. Rodr. Cilesalpineareale.

Jatobá da Sierra. Gebirg's J. Hymenaea Coreana Barb. Rodr. Casalpineacear.

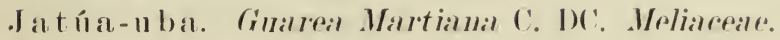

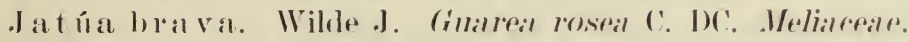

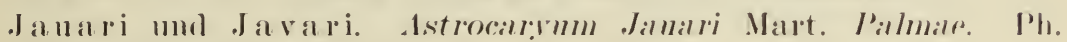
Rrksch., 1888, ю. 26:3.

J a ína. Solammm pteleaptolimm Sendt. Solinarrear.

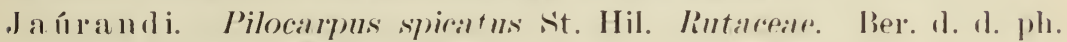
(ies., 1899 , р. 334.

Jebarí. Vide Cupaubà-rana.

Jembé. Nationalspeise im Staate Minas. Gesilzenes Schweinefleisch mit Quimgombófrucht und Maismehl.

Jeneúna. Vide Canna fistula.

Jenipabo, Jenipapo. Vide Genipabeira.

Jenipapada. ('onfekt der Genipafrucht.

Jenipapinha. Kleine Genipafrucht. Torognal Solloanal Sichum.

Ruliacease.

Jenipapo bravo. Wilde Genipapo. I Tocoyma tormosia sibum. Jenipapo do campo. Steppen (i. j Rubiaceate.

Jenipapo do nato. Ulwald (i. Tocorna hrasilimsis Mart. Rubiaceae.

Jenipapo miudo. Kleine G. Virle Jenipapinha.

Jequerioba. Vile Juciri.

Jequirity. Ibrus precatorins L. Papilionareale.

Jequitibí. Conratari romestica Mart. Mortarear.

Jequitibá de agulheira. Nadelbïchsen J. Vide Jequitiba rermelha.

Jequitibá assí. Grosse J. Lerrthopsis rufescens lig. Mrrtarear. Zeitsch. d. a. ister. Apt.-Ver.. 1868.

Jequitibá branca. Weisse .J. Conratari legalis Mart. Mrrtarear.

\%eitsch. d. a. ister. Apt.-Ver., 1868.

Jequitibai cedro. Vide Balata.

Jequitibá rosa. Conratari macrocarpa Mart. Myrtaceale.

Jequitibá vermelha. Rothe J. Comratari estrellensis Radd. Myr-

taceae.

J equitin-guassí. Tupybenennung fïr fructo de sabao.

J eram a taia. Virle Girimato. 
Jerema. Vide Jurema branca.

Jerataca und Jeratacaca. Vide Manacã.

Jerebita. Vide Mandureba.

Jerico. Aristolochia hrasiliensis Mart. et Zuce. Aristolochialcante.

Ph. Rdsch., 188:3, p. 181. Vide auch Angelicó.

Jeriva. Vide Baba de boi. Ph. Ridsch., 1888, p. 90.

Jetahy. Hymenaea Conrburil L. Caesalpineaceae. Arch. A. Ph., 82, p. 95.

Jetahy in Minas. Hymenaea Martiana Hayn. Caesalpineaceate.

Jetahy in Parà. Cynometra Sprnciana Benth. C'aesalpineareae.

Jetahy in Pernambuco. Hymenaeal stignorarpa Mart. Carsalpineareae.

Jetahy in Piauhy. Hymenaea stignocarpa Mat. var. pubescens Benth. Caesalpineaceap.

Jetahy-peba. Glatte J.

Jetahy-peba assí. Grosse glatte J. | Varietäten von Jetahy-peba mirim. Kleine glatte J. \ Hymenaeal Conrbaril L. Jetahy preto. Schwarze.J. Hymenaea eriogyne Bth. Caesalpineacerp. Jetaiba. Vide Jetahy.

Jetai-cica. Harz von Jetahy.

Jetica und Jetuca. Vide Batata doce.

Jeticucú. Ipomoea marrella Meissn. et I. hederarea L. Convolvulaceas.

Jetivy. Gegohrnes Getränk der Indianer von süssen Kartoffeln, Honig etc.

Jetua-rana. Vide Batata doce.

Jevura. Wird Phaseohns nana benannt, wenn zur trockenen Jahreszeit gepflanzt.

Jigoio. Costus igneus N. E. Brown. Zingiberaceane. Ph. Rdsch., 1893, p. 289.

Jijijapa und Jijipapa. Carludovica palmata S. et F. Cyclanthacers. Ph. Rdsch., 1893, p. 134.

Jilo. Vide Gilo. Afrikanische Benennung.

Jimbelé. Benennung im Staate S. Paulo für Canjica.

Jiquiri und Jiquirioba. Vide Juciri.

Jiquitaia. Reisegewiirz der Indianer. Masse von Capsicum barcatum und Mandioceamehl.

Jiracá und Jiraracá. Vide Jararacá mirim.

J Jirim ú und Jurum ú. Tupibenennung fïr Abobora grande. 


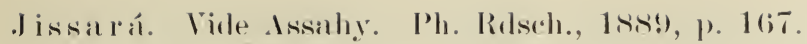

Jitalı amarello. Vide (iitaly amarello. IBer. 1. d. ph. Ges., $19(0), 1 \% .12: 3$.

Jito mul .Jitaiva. Vide (Xito in Pernamburo.

Jituiva. Vide Gito.

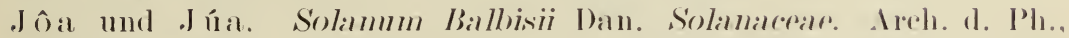
89, p. 247. I) 'Tupinamen Júa, Jôa. C'ui, Gíi. Gía fiir jerle fleischige Frucht.

Jôa der Nordstaaten. Solammm agrarinm Stend. Solanareare.

Jô a ma rella. Gelbe.J. Solammm ambrosiarmm Vellos. Solamarrar. Jôa a rebenta. Vide Arrebenta cavallo.

Jôa capote. Mantel J. Mhisalis pubescens L. Solanareane.

Jôa-colré-catinga. Stinkende rambe .J. Arrisial semirrenata var. repanclia Mart. Myrtareare.

Jôa da rosta. Kïsten .J. Vide Arebenta boi.

Jôa grande. Grosse J. Vide fructo de gambá.

Jôa garande do mato. Wilde grosse J. Vide Tuha de gambá.

J ôa manso. Lyeropersirum cerasiforme Dun. Solinirrar.

Jôa do Norte. Vide Melancia da praia.

Jôa-túa. Vide Jurumbeba do Norte.

Jôa-y. Virle Jôa.

Jôa-nesia. Vide Inda-assí.

Joao Gomes. Portularea mueronatal link. Portularcareale. Pli. Rev., 1896, p. 1.;).

J oao molle. I'isonia tomentosal Casalr. Ierctaginareap. Plu. Rev., $18.96,1) .81$.

Joao Puca. Essbale Waldfucht in Maranhao.

Joa pitanga. Vide Jeticúcí.

Joazeiro und Juazeiro. Zyzyphus joazeiro Mart. Rhammareate. Arch. d. Ph., 89, p. 247.

Jobotá. Vide Jabotí.

Jocará. Vide Jucoarí.

J oeirana. Acaria adharens Bth. Mimosareate.

Joeirana branca. Weisse J.

Joeirana preta. Schwarze .J. $\}$ Bauholz. Lanrareae.

Joeirana verinella. Rothe.J.

Jonquilho und .Junquilho. I Lansbergia junrifolia Klatt. IridaJonquitho do campo. i reate. Plı. Risch., 1892, 1) 132. Jorova. Vide Maria Rosa. 
Jí. Bactris marrocarpa Wall. Palmae. Ph. Rdsch., 1888, pp. 202 11. 264. Astrocarinm acaule Nart. A. humile Wall.

Juá-assí.
Jú-uva.

Juá-poca. Vide Camapú.

Juati. Virle Arrebenta carallo.

Jubai. Vide Tamarindeiro.

Jubati. Vide ('oqueiro Jubati. Ph. Rdsch., 1888, p. 12!).

Jubeba. Solanum spectabilis. Steud. Solanaceas.

J и́ca. C'aesalpinia Gardneriana Bth. C'alesalpineareale.

J u capé. Vicle Sapè.

Juciri. Solanum Juciri Mart. Solanaceae.

Jucury-assí. Vide Junqueira-assú.

J и́-i. Bactris arundinaca Trl. Palmae. Ph. Rdsch., 1888, p. 202.

J í-i-úba. Bauholz in Parà.

Juiva. Astrocarrum campestre Mart. Palmae. Ph. Ridsch., 1888, p. 264.

Jujúba. Zyzyphuss Jujuba Gaertn. Rhamnaceae.

J $u \mathrm{mbeba}$. Cereus variahilis Pfeiff. ('actareap. (Von kum-ausgestreckt, peba-glatt.)

Junça. Crperus elegans Schrad. Crperaceae. Ph. Rdsch., 1894, p. 187 .

J un ra a grande. Grosse J. Crperus articulatus Schrad. Crperaceae. Ph. Rdsch., 18:94, p. 187.

Junça piquena. Ǩleine J. C'rperus gracilescens R. et S'chult. C'Yperaceate. Ph. Rdsch., 1894 , p. 187.

Junço. Vicle Calamo bravo. Ph. Rdsch., 1894, p. 188.

Junço de cobra. Sichlangenrohr. Vide Calamo da terra.

Jundiahyba. Terminalia glabresrens Mart. Combretareate.

Junqueira. Evolvulus filipes Mart. Convolvulaceae.

Junqueira-assí und Juqueira-assí. Grosse J. Entarlil polystachya DC. Mimosaceae.

Junta de cobra. Schlangengelenk. Beloperone nodicaulis N. ab. E. Acanthaceae.

J u u te i r o. Cielenkpflanze. Dichorisandra tuherosa P. Commelinarear. Ph. Rdsch., 1892, p. 257.

Jupalla. Eryngium paniculatum Car. I'mbelliferale.

Jupana. Heilmittel in Parì.

Jupeba. Vide Jubeba. 
Jupicay. Vide Herva de impigem.

Jupiede und .Jupiedi. Yyris laxifolia Mart. Yrvidareale. P'r.

Rilsch., 18!)2, p. $1(i 4$.

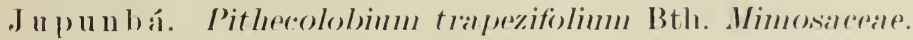

Juqua-rana mul .J urrana. Bamholz in Permambnoo).

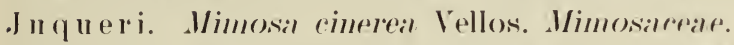

J uqueri manso. Neptmuial olerareal Lour. Mimosiaceate.

J uquerioba. Vide Juciri.

J uquery-onano. ! Ville Arriozes.

J nq u irianano. i Vile Mrriozes.

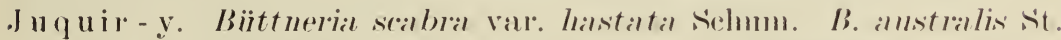

Hil. B. filipes Mart. ('Tupi jú-Stachel, ker-betäubend, y-klein.)

sterenliarpale. Ber. (. a. ph. (res., 1!)()(), p. 12:3.

J urati. Vide P'epepena.

J urema. Mimosa verrucosal I3th. Mimosialrarr.

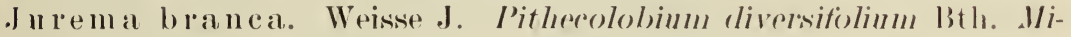

mosilceile.

J urema bordata. Geschmïckte J. Vicle Inga bordata.

Jurema preta. Schwarze.J. Acilcial glomerosil Bth. Mimosilmale. Jurema de remo. Ruder .J. Vile Jurema bramca.

J ureva. Vide Maria Rosa.

Juricurar̀. Wundheilpflanze in Mlagoas.

Juripari-iba. Teufelsbaum in Imazonas. Strochnos toxiferal Bth.

Loganiaceate.

Ju ripari-roka. Teufelskralle. Clussia ralumnaris Engl. Guttiferar. Juripari-pirera. Schuppige Teufelspflanze. Strrchnos subcorlatil

Spruc. Loginiaceae.

J uripeba. Solanum insidiosum Mart. Solanalreate.

Juripeba in Pernambuco. Solanum pantrulatmm L. Símlanareat.

Areh. d. I’h., 102, p. 16.5.

Juripeba in Par̀̀. Solanum. Inrijebar Rich. Solanaceate.

Juripeba do campo. Steppen J. Solannm firstigiatum Willı.

Solanaceae.

Juripeba mirim. Kleme J. Solanmm paniculatum L. Varr. arenti-

lobum Sendt. Solanaceate.

J uriquity. Vide Jequirity.

Jurity-ubim. Vicle Assai-rana.

Jurubeba da pharmacia. Otficinelle J. Vide Juripeba.

Jurujúba. Verbena chamaedryfolia .Juss. Verbenacear. 
Jurumbeba. Solanum subscandens Vell. Solanalceate.

Jurumbeba de P'ernambuco. Vide Juripeba in Pernambuco.

Jurumbeba in Rio de Janeiro. Vide Juripeba.

Jurupeba. Vide Jubeba.

Jurupeba brava. ,

Jurupetinga.

\} Solanum megalonyx Sendt. Solanaceale.

J urupetinga mir im. Kleine J. Solannm asperum Vahl. Solanacear, J uríp a ri-heua. Teufelsstachel. Vide Flecha do diabo. I'h. Rdsch.,

1888, p. 203.

Juruté. Essbare Frucht einer ('ordia.

Juruva. Vide Baba de boi.

J u tay. Vicle Jetahy.

Jutay merim. Kleine J. ， Hymenama microphylla Barb. Rodr. Jutay pororoca. Platyer J.j Caresalpineareate.

Jutay-peba. Gilatte J. Calesalpineaceate.

Jutahy. Vide Tamarindeiro.

Juta y-rana. Falscher J. ('rudyal Parivoal DC, P'alpilionaceate. Jutica-rana. Vide Batata brava. Ph. Areh., 1898, p. 188.

Jutúa-ïba. Guarea Martianal C. DC. Ber. d. d. ph. Ges., 1901.

J u veva. Solanum insidiosun Mart. var. pubescens Sendt. Solanacear.

Kaa-boby. Tupiname fïr Anil.

Kaachaby. Vide Urumbeba.

Kaa-có. Tupiname fïr Sensitiva.

Kaa-hyra. Tupiname fïr Mata pasto.

Kaboanta. Vide Camboita. Ber. d. (l. ph. (ies., 1898, p. 4:3:3.

Kabure-yba. Tupiname fiir Cabureiba.

Kachang. Negerbenennung fïr Guanto.

K a mba-namby. T'upiname fïr Tamburi.

Kambuy de restinga. Vide Comandahiba.

Kanali-cucher.y. Tupiname fïr Craveiro de Maranhao.

Karacalla. Euphorbia Tirucalli L. Euphorbiaceate. Von Afrika importirt.

Karagalla. Norantea guianensis Mubl. Marcgraviaceate.

Karapa. Vide Carapa.

Kilo. Chrysocona annara Schum. Compositae. Negerwort, von den

sclaven eingefïhrt und verwildert.

Kisafí. Negerbenennung für Vrucú.

Kiwjá. Tupiname fïr Paó cravo. 
Komandaliyba. Vide Comandahiba.

Kopú. Goupea tomentosa Aubl. Celastrinaceae.

Koyhab. Aus diesem Tupiworte ist durch Corruption die portugiesische Benennung Guiabeira oder (Hoiabeira entstanden.

Kuarubá-uva. Vochysia olasyantha Warm. Vochysiaceae.

Ku ambri. Tupiname für Carapicho.

K umakaa-y. Claytonia odorata Barb. Rodr. Portulacaceae.

Ku mibarí. Vicle Cumarú.

Kupuassí. Tupiname fïr Cacalo sylvestre od. Casertão. Ber. d. d. ph. Ges., 1900, p. 119.

Kyinha. Tupiname für Capsicum.

Labaça. Rumex brasiliensis Lenk. Polygonaceae. Ph. Rdsch. (N. Y.), 1895, p. 34 .

Lab-lab. Qualea rosea Aubl. Vorhysiaceae.

Lacre branco. Weisser Lack. Melastomaceae.

Lacre vermelho. Rother Lack. Vide Caaopia do campo und

Caaopia. Ber. d. d. ph. Ges., 1897, pp. 235, 236.

Lagarteiro. Möbelholz im Staate Parahyba.

Lagrima de moça. Mädchenthräne. Vide Lirio suzena.

Lagrimas da Nossa Senhora. Mutter Gottesthräne. Coix larrima L. Gramineae. Ph. Rdsch., 1894, p. 166.

Lagrimas de Santa Maria. Vide Arriozes.

Lanceta. Eclipta alba Hasck. Compositae. Schlangenantidot. Arch. d. Ph., 1862, p. 137.

Lanceta in Rio Grande do sul. Solidago microglossa DC. Compositae.

Landirana. Auch Jandirana. Bauholz.

Lantim und Lanty. Viłle Jacaré-huiba.

Lapacho. Vide Paó d'areo.

Lappa vermelha. Rothe Klette. Sicla densiflora Mock et Arn.

Malvaceae. Ber. d. d. ph. Ges., 1900, p. 265.

In Minas Xanthoxylum acutifolium Engl. Rutaceae. Ber. d. d. ph. Ges., 1899, p. 345.

Laranja boceta. Schachtelorange. ('itrus melitense Risso. Rutaceae. Ber. d. d. ph. Ges., 1899, p. 355.

Laranja da China. Chinesische O. Citrus aurantium Risso, Ru. taceae. Ber. d. d. ph. Ges., 1899, p. 355. 
Varietäten: Laranja branca. Weisse 0 .

Laranja Cametá. Cametá 0 .

Laranja Campista. Campos 0 .

Laranja Lima. Limonen 0 .

Laranja Natal. Weihnachts $O$.

Laranja oro. Eierorange.

Laranja péra. Birnen O.

Laranja prata. Silber 0 .

Laranja de saude. Gesundheits O.

Laranja selecta. Vorzügliche $\mathrm{O}$.

Laranja de umbigo. Nabel $\mathrm{O}$.

Laranja tangerina. Kleine Orange. Citrus deliciosa Risso. Mit den Varietäten:

Laranja cravo. Nelken 0 .

Laranja mexirica. Klatschschwester O.

Laranja da terra. Einheimische O. Citrus vulgaris Risso. Mit den Varietäten :

Larania da terra branca. Weisse einheimische 0 .

Laranja da terra doce. Süsse einh. O.

Laranja turanga. Citrus pomum Adami Risso. Mit den Varietäten:

Laranja turanga azeda und Larangao. Grosse 0 .

Laranja do mato. Waldorange. Allophylus sericeus Radlk. Silpindaceae.

Larania Cacupari. Bakupari O. |Laetia suaveolens Bth.

Laranja miuda do mato. Kleine wilde O.f Flacontiaceae. Ber. d. d. ph. Ges., 1899, p. 167.

Larangeira a marella. Gelber Orangenbaum. Rutaceae. Bauholz. Spec. Gew. 1,148 .

Larangeira brava. Wilder O. Xanthoxylum monogynum St. Hil.

Rutaceae. Ber. d. d.ph. Ges., 1899, p. 345. Vide auch Tinguciba.

Larangeira do mato. Wald Orangenbaum. Esenbeckia felurifuga

A. Tuss. Rutaceae. Ber. d. d. ph. Ges., 1899, p. 336. Spec. Gew. 1,076 .

La ranginha. Vide Cabacinha.

Laranginha in Pernambuco. Kleine Orange. Vide Jenipapo bravo.

Laranginha in Rio. Vide Limaosinho.

Laranginha brava. Wilde kleine 0 . Vide Larangeira brava, 
Laranginha do campo. Kleine Steppenorange. Vide Bacopari da capoeira.

Laranginha do mato. Kleine Waldorange. Monnina cuneata St. Hil. Polygalaceae.

Laranginho. Zuckerbranntwein mit Orangenschale aromatisirt.

Larangitas de Quito. Solanum Quiloense Lam. Solanaceae.

Lascadinha. Bauholz in Alagoas.

Lava prato. Tellewascher. Solanum torvum Schw. Solanaceae.

In den Nordstaaten Cassia quinqueangulata Rich. Caesalpineacea.

In den Südstaaten vide Mangerioba.

Lechetriz. Vide Maleiteira.

Legação. Vide Salsaparilha.

Leitariga. Vide Maleiteira.

Leite de coco. Der ausgepresste Saft des geriebenen Fleisches der Cocosnuss. Wurmmittel.

Leiteira. Milchbaum. Tahaemaemontana Salzmanni DC. Apocy-

naceae. Auch Maleiteira. Arch. d. Ph., 1855, p. 94.

Leiteira de graõ de gallo. Hahnhoden-Milchbaum. Ambelania laxa Müll. Arg. Apocvnaceae.

Leiteira do mato. Wald Milchbaum. Vide Massaranduba.

Leiteira do Mato Virgem. Urwaldmilchbaum. Sideroxylon

crassipedicellatum Mart. Sapotaceae. Bauholz. Sp. Gew. 1,222.

Lentilha d'agua. Wasserlinse. Vide Flor d'agua. Ph. Rdsch., 1893.

Levante. Dichondra macrocalyx Meissn. Convolvulaceae.

Liam bo. Vide Pango.

Licari. Vide Craveira de Maranhao.

Licari-kanali. Vide Paó de cravo.

Licorana und Licurana. Vide Urucú-rana.

Ligadeira. | Klebepflanze. Phrygilanthus cuneifolius Don.

Liga grudadeira. $\int$ Loranthaceae. Pharm. Arch., 1900, p. 143.

Liga-liga und Liga paó. Phoradendron Perottetii Eichl. Loranthaceae.

Lig a-osso. Knochenleimer. Dorstenia brasiliensis Lam. Urticacer. Ph. Rdsch., 1891, p. 291.

Lima doce. Süsse Limone. Citrus limetta Risso Rutaceae. Ber. d. d. ph. Ges., 1899, p. 360.

Lima da Persia. Persische L. Citrus limettia maior Risso Rutacea. Ber. d. d. ph. Ges., 1899, p. 360 . 
Lima do umbigo. Nabel L. Citrus limetta minor Risso Rutaceae. Ber. d. d. ph. Ges., 1899, p. 360.

Limao und Limao azedo. Saure Limone. Citrus limonum Risso

Rutaceae. Ber. d. d. ph. Ges., 1899, p. 360.

Limao bravo und L. francez. Citrone. Citrus medica Risso

Rutaceae. Ber. d. d. ph. Ges., 1899, p. 360.

Limao bravo. Wilde Limone. Citriosma apiocyce Mart. Monimiaceae. Ber. d. d. ph. Ges., 1896, p. 94.

Limao do mato. Vide Jasmin do mato.

Limaosinha. Kleine Limone. Acanthocladus brasiliensis St. Hil. Polygalaceae.

Limo. Flechten, welche die Felsen bekleiden.

Limo do rio. Algen in den Flüssen und Bächen.

Limoeira do nıto. Waldlimonenbaum. Vide Catagúa. Ber. d. d. ph. Ges., 1899, p. 339.

Linda flor. Schöne Blume. Mutisa speciosa Hook. Compositae.

Lingua de araçari. Vide Lingua de tucano.

Lingua de boi. Ochsenzunge. Vide Cipo de escada.

Lingua de coelho. Kaninchenzunge. Elephantopus riparius Gard. Compositae.

Lingua de cutia. Agutizunge. Sida linearifolia St. Hil. Malvaceae.

Ber. d. d. ph. Ges., 1900, p. 266.

Lingua de fiú. Fadenzunge. Vide Chá de frade.

Lingua de sapo. Froschzunge. Piperomia transparens Miq.

Piperaceae. Ph. Rdsch., 1894, p. 240.

Lingua de tatú. Gürtelthierzunge. Heilpflanze im Staate Bahia. Lingua de teiń. Eidechsenzunge. Vide Herva de lagarto in S. Paulo. Lingua de tucano. Pfefferfrassvogelzunge. Eryngium tristis Cham.

Umbelliferae. Arch. d. Pharm., 1857, p. 240.

Lingua de vacea. Kuhzunge. Chaptalia nutans Hensl.; in S. Paulo Chaptalia integrifolia Bak. Compositae. Arch. d. Pharm., 1857, p. $111: 1860$, p. 168 .

Lirio. Vide Tukyra.

Amarillis regia L. Amaryllidaceae. Ph. Rdsch., 1893, p. 135.

Lirio d’agua. Wasserlilie. Vide Agoapé. Ber. d. d. ph. Ges., 1897, p. 284.

Lirio amarello do campo. Gelbe Steppenlilie. Allophia Sellowiana Klatt. Iridaceae. Ph. Rdsch., 1892, p. 132.

Lirio cardino. Distellilie. Iris Xyphium L. Iridaceae. Kultivirt. 
Lirio da India. Indische Lilie. Vide Cinnamoni. Ber. d. d. ph. Ges., 1901.

Lirio do mato. Waldlilie. Cypella coerulea Seub. Iridacere. Ph. Rdsch., 1892, p. 132.

In Alagoas Amaryllis silvatica Mart. Amarylliclaceae.

Lirio roxo. Violette Lilie. Lanshergia purgans Klatt. Iridaceae. Ph. Rdsch., 1892, p. 132 .

Lirio suzena. Hedychinm coronarium Koen. var. maximum Eichl. Zingiberaceae. Ph. Risch., 1893, p. 287.

Lirio verde. Grüne Lilie. Cypella Northiana Klatt. Iriclaceae. Ph. Rdsch., 1892, p. 133.

Litschi. Nephelium Litchi Don. Sapindaceae. Kultivirt.

Lixa. Polirpflanze. Equisetum gigalntenn L. Equisetaceae und Lippia urticoides Steud. Verbenaceae. Areh. d. Ph., 1862 , p. 131. Lobo-lobo. Alsodeia physiphora Mart. Violaceae. Ber.d. d. ph. Ges., 1897, p. 104.

L obo-lobo. Vide Arapa baca.

Loco. Plumbago scandens L. Plumbaginaceae. Ber. d. d. ph. Ges., 1901.

Loiro. Lorbeer. Cordia hypolema A. DC. Cordiaceae.

Longana. Nephilium longana Hook. Sapindaceae. Kultivirt.

Losna de Maranhao. Wermuth im Staate Maranhao. Ambrosia microcephala DC. Compositae.

Loucura. Lagerströmia nitida L. Lythraceae. Eingeführter Zierstrauch.

Lourel. Nectandra laurel Kl. Lauraceae.

Louril de Paraná. Nectandra angustifolia var. falcifolia Nees. Lauraceae. Pharm. Arch., 1898, p. 27.

Louro. Lorbeer. Vide Loiro und Carúa-iba.

Louro abacate. Bauholz in Pará und Amazonas. Lauraceae.

Louro amarello. Gelber Lorbeer. Persea splendens var. chrysophylla Meissn. Lauraceae. Pharm. Arch., 1898, p. 1.

Louro amarello cheiroso. Wohlriechender L. Aydendron tenellum Meissn. Lauraceae. Bauholz. Sp. Gew. 0,091.

Louro amarello de cheiro. Vide Canella de cheiro. Pharm. Arch., 1898, pp. 12 u. 13.

Louro batata. Knollen L. Vide Canella batata. Pharm. Arch., 1898, p. 32.

Louro amargoso. Bitterer L. Vide Canella preta da serra. Pharm. Arch., 1898, p. 24. 
Louro anhoiba. Vide Annuiba do brejo.

Louro bahiana. Bauholz in Bahia. Lauraceae. Sp. Gew. 0,836.

Louro besuntão. Stinkender L. Vide Canella fedorenta. Pharm.

Arch., 1898, p. 27.

Louro besunto. Schmutziger L. Vide Louro amarello.

Louro branco. Weisser L. Persea alba Nees.; Oreodaphne suaveo-

lens Meisn. In Paraná Oreodaphne acutifolia Nees.; Oreodaphne velutina Nees. Lauraceae. Pharm. Arch., 1898, pp. 1 u. 19.

Louro canella. Zimmtlorbeer. Bauholz in Pernambueo. Lauracex. Louro canga de porco. Schweinsjoch L. Persea alba Nees. Lauraceæ. Louro cedro. Cedernlorbeer. Bauholz. Sp. Gew. 0,688.

Louro cergeira. Kirschenlorbeer. Vide Gingeira brava.

Louro chu in bo. Bleilorbeer. Bauholz.

Louro faia. Buchenlorbeer. Bauholz.

Louro de folha larga. Vide Canella de folha larga. Pharm. Arch., 1898, p. 24.

Louro giboia. Schlangenlorbeer. Bauholz.

Louro manteiga. Butterlorbeer. Bauholz. Sp. Gew. 0.753.

Louro marfim. Vide Marfim.

Louro do mato virgem. Urwald L. Vide Fructo de caxinglé.

Pharm. Arch., 1898, p. 20.

Lo u ro pardo. Brauner L. Cordia alliodora Cham. var. tomentosa

Fresc. Cordiaceae. Bauholz. Sp. Gew. 0,353-0,401.

Louro passarinho.

Louro periquito.

Louro pimenta.

Louro preto. Schwarzer L. Cordia obscura Cham. Cordiaceae.

Nectandra mollis Nees. var. villosa Meisn. Lauraceae. Pharm.

Arch., 1898, p. 21 . Vide auch Canella preta da serra.

Louro sylvest.re. Wilder L. Nectandra canescens Nees. Lauracex.

Pharm. Arch., 1898, p. 20.

Louro thi. Auch Carvalho de capoeira. Nectandra leucantha Nees.

var. attenuata Meisn. Lauraceae. Pharm. Arch., 1898, p. 22.

L o uro verdadeiro. Aechter Lorbeer. Vide Canella fedorenta.

Louro vermelho. Rother L. ) Bauholz. Sp. Gew. 0,622-0,848.

Louro virote. Bolzen L. $\quad \int$ In den Nordstaaten.

Luminaria. Allerlei. Confect von Cocosnussfleisch etc.

$\left.\begin{array}{l}\text { Luzeiro. Leuchte. } \\ \text { Luzetro. Wulfsmilch. }\end{array}\right\}$ Vide Maleiteira.

L y copodio da terra. Einheimischer Bärlapp. Vide Froco. 
Mabouia. Morisonia americana L. Capparidaceae.

Macaa de cobra. Schlangenapfel. Vide Araticum do Crejo. Ber. d. d. ph. Ges., 1897 , p. 455 .

Macaa do mato. Waldapfel. Lucuma Sapotaceae. Ph. Rilsch., 1888, p. 33.

Maca-apa-ipí. Vide fructo de sabao.

Maca-ca-cipo. Heilpflanze im Staate Amazonas.

Maca-ca-uba und ïva.

Maca-ca-uba branca. Weisse M. Baulölzer in den Staaten Maca-ca-upa do matto. Wilde M. Amazonas und Parà. Maca-ca-uba preta. Schwarze M. Maca-ca-uba da varzea. Thal M. Maca-ca-iba-ipon. Indianische Benenumg für Buranhem. Ph. Rev., 1897, p. 454 .

Macachy. Guaraniname für Trevo rasteiro.

Macaiba und Macaub. Acrocomia slerocarpa Mart. Palmae. Ph. Rdsch., 1889, p. 34 .

Macaiauba. Bauholz in Parà. Leguminosae.

Macajuba. Indianische Benennung für Mandiocea.

Macaluma. Vide Bacoparido campo.

Macambira. Bromelia laciniosa Mart. Bromeliaceae. Ph. Rdsch., 1895 , p. 237.

Macambira branca. Weisse M. Cereus Hiabelliformus Mill. Cactaceae.

Macambira de cachorro. Hunde M. Cereus Hildemannidnus K. Sch. Cactaceae.

Macambira de flechas. Pfeil M. Vide Macambira.

M a.caná. Kriegskeule der Indianer, gewöhnlich von Eisenholz.

Ma caqueiro. Guarea multiflora A. Juss. Meliaceae. Ber. d. d. ph. Ges., 1901.

Ma caxéra. Tupybenennung der Aipim (Mandioca). Ph. Rdsch., 1886, p. 57.

M acega. Das hohe Grass des Camposgebietes, wenn trocken zum Brande.

Macella. Kamille. Gnaphalium macella Vellos. Compositae; Achyrocline saturoides DC. Compositae.

Macella branca. Weisse K. Vide Macellinha.

Macella do campo. Steppenkamille. Achyrocline saturoides DC. var, mathiofolia Bak. Compositie. 
Macella do mato und braro. Wilde K. Achrrocline saturoides DC. var. vargasiana Bak. Compositae: Telanthera brasiliana IIoq. und $T$. ramosissima Moq. Amaranthaceae. Ph. Rdsch., 1895, p. 89.

If acella do sertao. Wiisten $\mathrm{K}$. Achrrocline saturoides DC, var. albicans Bak. Compositae.

Macella da tabobeira. Hochebenen K. Convza Blanchetii Bak. Compositae.

Ir acella da terra. Einheimische K. Egletes riscosa Lep. Compositae.

Macellinha. Kleine K. Gonphrena jubata Moq. Amaranthaceae. If ehicha und Maxixa. Cucumis anguria L. Cucurbitaceae. Kultivirt.

M a cohim-kaka. Brennkraut. Dalechampia Peckoltiana Müll. Arg. Eupliorbiaceae.

Macoucou und Macúcú. Chrrsophyllum macoucou Aubl. Sapotaceae; Ilex macoucoua Pers. Ilirinaceae.

M a cujá. Couma rigidia Müll. Arg. Apocrnaceae.

Macujé und Macuji. Sapotareae. Bauholz.

Il a cúma. Vide Coqueiro do campo.

М а с upi. Vide Curúa-tinga.

Ma depueira. Graefenrieila crsptocarpa Fr. All. Melastomaceae.

II a re cravo. Nelkenmutter. Epaltes hrasiliensis DC. Compositae. Madre silva. Waldmutter. Alstroemeria carrophyllea Jacq. Alstroemeriaceae.

Madre silva do mato. Wilde W. Alstromeria Cunha Vellos. Alstroeneriaceae. Ph. Rdsch., 1892. p. 162.

Ma dupitiú. Arachis tuberosa Bong. Papilionaceae.

Maduro. Benennung des gegohrenen Zuckersaftes ror der Destillation.

I a e boa. Hülfreiche Mutter. Titis sulcicaulis Kak. Ampelirlaceae. Tide auch Abutua.

Irae de familia. Familienmutter. Vide Margaridinha.

Mae de sapateiro. Schustermutter. Borreria argentea Cham. Rubiacease.

Ma fahú. Grosse Cajúfrucht in den Nordstaaten.

Il agnolia brara. Wilde M. Clusea Arrudea Blanch, et Trian. Guttiferae. Ber. d. d. ph. (Ges., 1897, p. 239. 
Magnolia do mato. , Einheimische M. Vide Canella do brejo. Ilagnolia da terra. $\quad$ Rer. d. d. ph. (ies., 1896. pp. 157, 159). Magnolia miuda. Keine M. Michelia camparal L. Magnoliacear. Kiltivilt.

Mahйbа. Mahnreal grandiflora Fr. All. Ginttiferate.

Majangue. Indianerspeise von Schildkröteneiern, Mandioccamehl, etc.

Ma imbá. Schlingpflanze. Bignoniaceate. Mrch. ı. I’h., 1860, p. 166.

Ma imbí. Vitis rorecineal Bak. Ampelislaceate.

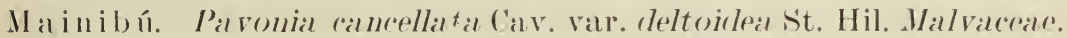
Ber. (1. (1. p)!. (iess., 19)()(). p. 271.

II a iocrana. Indianische Benemmung für Algorloeira brava.

Macachy. Guaraniname fïr Oxalis corniculatal L. Oxalidalcoate. Ber. (1. d. ph. (ies., 1899, ). 46 .

Makokim-eaka. Leucaena glancal Bth. Mimosalrear.

Mal casado und Mal cassí. Gebäck ron Tappocea und Cocosmussmilch.

Malambó. Vide ('asca d'anta. Ber. d. d. ph. Ges., 189), p. 161.

Ilaleiteira. Schlimmer Milchbam. Énphorbia papillosal St. Hil. Emphorlialreale.

II alhado. Vide Incenco (I'ittosprorum).

Malicia de gato. Katzenbosheit. Vide Melosa de gato.

Malicia de homem. Mämnerbosheit. Vide Calmmbi.

Ilalicia de muller. Weiberlist. Mimosal invisa L. Mimosaceac. Vide Sensitiva.

Ma lmequer. Ringelblume. Lantana mixta L. Verbenacrar. Mreh. d. Ph., 1857, Bal. 91, p. 2:39.

Malmequer am a rello. Gelbe R. Vide Malmecuer dos jardins.

II a l 1 equer do brejo. Sumpe R. Aspilia foliarea. Bak. Compositae. Malmequer de campina. Wiesen R. Wedelia paludosal DC. var. vialis Bak. Compositale.

II almequer grande. Grosse R. Vide Andrequice.

Malmequer dos jardins. (iarten R. C'hrysanthemum carinatum Shlı. und Ch. coronarium L. C'ompositale.

Malmequer sinha de campina. Kleine Wiesen R. Epipactis camporum? Orchiclaceate.

II alva und Malva da C'otica. Apothekenmalve. Malva sylvestris L. var. Mauritiana Bois. Malvaceate. Ber. d. d. ph. Ges, 1900, p. 265. Malva de bexiga. Harnblasemmalve, Vide Malva diuretica. Ber. l. d. ph. Ges., 1900 , p. 265. 
Malra branca de campina. Weisse Wiesenmalve. Sidia Gaudichiana G. Don.: Gava Gaudichiana st. Hil. Malvaceae. Ber. d. 1. ph. Ges., 1900, p. 265.

Malra branca macia. Weisse zarte M. Sirda cordifolia L. Malvaceae. Ber. d. d. ph. Ges.. 1900. p. 265.

Malra brava. Wilde M. Abutilon melanocarpun st. Hil. Malvaceace. Ber. 1. d. ph. Ges.. 1900, p. 265.

II a Ira do campo. Steppen M. Kielmegra speriosa St. Hil. Guttiferae. Ber. d. d. ph. Ges.. 1s97, p. 229.

Malra diuretica. Paronia sidifolia Kth. var. dintetical liirk. Malraceae. Ber. d. d. ph. Ges.. 1900, p. 270.

If alra de folha grande. Grossblättrige M. P'arouial viscosil J. Juss. Malraceate.

Malra grande. Grosse M. Vide Rachador.

Malra de Judeo. Judenmalre. Vide Vassonra (Corchorus).

Ifalra lanceta. Lancetten I. Sïld spinosal L. var. angustifolial Gris. Malvaceae.

If lra macaa. Aepfelriechende M. Pelargonium orloratisimum Ait. Geraniaceae. (iartenptlanze.

Malva d’óuralo. Gold M. Gááa aurea st. Hil. Mallaracae.

Malra rosa. Rosen M. Hibiscus rosal sinemsis L. Milliaceate.

Malra da terra. Einheimische M. Sïda glomerata Car. Malvaceate. If lraisco. Althea. Sphatralea cisalpina; Paronia malicophrilla Gürk. Malvaceae. Vide Guaxima macho.

Malraisco in Pernambuco. Arthante scrlopioides Miq. Pipera-

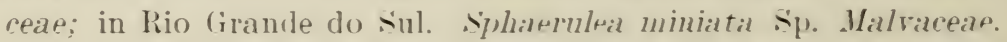

II avalistro. Ge treifte M. Sida rhombifolia L. Malrareat. Siehe diese Familie in den Ber. 1. 1. ph. Ges.. 1899. p. 265.

Ir a manarana. Benennung ter Indianer im staate Amazouas der Jaracatia.

Mamao femea. Weibliche.

I a mao macho. Männliche. Frichte ron Mamueira.

Il a mao melao. Melonen.

II a mao merim. Kleine Papayafrucht. Carica heterophy-llia Popu. et Endl. Caricaceae.

Ma mbú. Yegerbenennung für Bambì.

If a meluca. Bauholz im staate Alagoas.

If a ma de cachurra. Hündinzitze, Phollosalix formosus Be. Mrrtaceat. 
II a mma cocea. Springfruchtzitze. Errthoxyhm mamacoca Mart. Erythoxylaceae.

Il a mma de porca do campo. Sauzitze der steppe. Santhoxylum tuberrulatum Engl. Rutaceas. Ber. d. d. ph. Ges., 1899, p. 345.

II a mma de porca in Menàs. Sauzitze. Yanthoxylum cinerenm Engl. Rutaceas; in L. Paulo. Xanthoxylum latespinosum Engl. Rutaceae. Ber. d. a ph. (ies., 18!9), p. 345; in Rio de Janeiro. Machaeriun aculeatum Radd. Papilionaceas und Buranhem.

II a minha de cadella. Kleine Hïndinzitze. Rutaceae. Bauholz. Mamminha de cachorra. Höndinyitzchen. Brosimum Gaudichandii Tres. from longuis pednnculitil Trticaceap.

Mamminlıa de porca. Sauzitzchen. Xinthoxylum rhoifolium Lam. Rutaceae. Ber. d. d. ph. (ies., 1899, p. 346.

M a moeira. Papayabaum (Weiberbrust). Carica papara L. Caricacere. Ztschr. d. ̈̈ster. Ap. Ver., 1879, No. 24 und 2.,

Il a moeira do mato. Wilder P. Vide Jaracatia.

Mamoerinha. Kleine P. Carica quercifolia Solnn. Caricaceae.

Mamona. Ricinussame. Ricinus communis L. Enphorbiaceae.

Mamona branca. Wesser R. Ricinus communis L. var. genuinus Mïll. Arg. Euphorbiaceae.

Mamona branca de espinho. Weisser stachlicher R. Ricinus communis L. var. microcarpus Müll. Arg. Euphorbiaceae. Arch. d. Ph., 1857, Bd. 91, p. 249; 1860, Bd. 102, p. 159.

Ma mona vermelha. Rother R. Ricinus communis L. var. brasiliensis Müll. Arg. Euphorbiaceae.

Mamona vermelha de espinha. Rother stachlicher R. Ricinus commnnis L. var. radius Müll. Arg. Euphorbiaceae.

Mamona brava. Wilder R. Mabea fistuligera Mart. Euphorbiaceae. Mamona do mato. Wald R. Micrandra elata Mïll. Arg. Euphorbiaceae.

Mamotá und Mamoté. Indianische Benennung für Jaracatia.

II a mum und Mamunga. Gynandropsis pentaphylia DC. Capparidacexe. Vid. auch Lava prato do Norte.

Ma náca. Enterpe oleracea Mart. Palmae.

M a nacaa (Manacan). Brunsfelsia. Hopeana Bth. Scrophulariaceae. Ach. d. Ph., 1860, Bd. 102, p. 167; 1862, Bd. 110, p. 134.

Man acaa-an acon. Casselia integrifolia Ness. Verbenaceae.

II anacaa do mato. Wald.M. Nehlsonia Pohlii N. ab C. Acanthaceae. 
Ma nac aa-rana. Falsche M. Paypayroha grandiflor: Tul. V'iolaceae. Manacaa da serra. Gebirg's M. Brunsfelsia ramosissima Bth. Scrophnlariaceae.

Manacarú. Vide Mandacarú.

M a n ampansa. Vide Beijú, wenn mit Anis aromatisirt.

Manapusca. Mouriria princeps Naud. Melastomaceae.

Manaué. ('iebäck von Maismehl, Honig etc.

Mancinilha. Hippomane mancinellal. L. Euphorlialeeale.

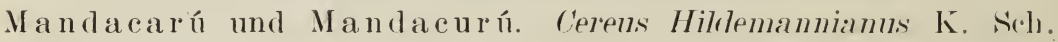

Cactaceae.

M a ndak. Vide Paratudo do mato.

Mandapúca. Vide Jaboticabeira cabellucla.

Mandarina. Citrus nobilis Lour. Rutaceale.

Mandiba. In den Nordstaaten Benennung fiir Mandioca.

M andibi. Vide Imbé miudo.

Mandibi das juntas. Knoten M. Vide Cipó Imbé.

Nandigii. Indianische Benennung für Baumwolle.

Mandiocahy. Didymopanax Morototoni Done. Araliaceae.

Mandiocea od. Mandiocea amargosa. Manihot utilissima Pohl

Euphorbiaceae; lat 30 Varietäten. Ph. Rdsch., 1886, pp.57-203.

Mandiocea. Aypin. Vide Aypim, hat 15 Varietäten. Ph. Rdsch.,

1886, pp. 57-20:3.

Mandiocea branca do mato. Weisse Wald M. Manihot Pohlii

Wawra; Manihot flabellifolia Pohl. Euphorbiareae. Plı. Rdsch.. 1886, pp. $57-20: 3$.

Mandiocea brava. Wilde M. Jatropha Pohliana var. divergens

Mïll. Arg. Euphorbiaceae; C'leome gigantea L. Cappariclareale.

Ber. d. d. ph. Ges., 1898, p. 41.

Mandiocea vermelha. Rothe M. Vide Mandiocea.

Mandiocea vermelha do mato. Rothe Wald. M. Manihot paviafolia Pohl. Enphorbiaceae.

Mandiocquinha. Kleine M. Manihot pubescens Pohl. Enphorbiaceae.

Mandiocquinha in Minas. Croton lobatus var. Manihot Müll. Arg. Euphorbiaceae.

Mandiocquinha bravo. Kleine wilde M. Vide Pimenta de macaco und Catinga do negro.

Mandiocquinha do campo. Kleine Steppen M. Vide Bolsa do pastor. 
Mandiocquinha falsa. Falsche kleine M. Vide Mandiocahy.

If a ndigŕ. Guaranibenennung fïr Algodoeira.

Mandobi, Mandubi, Mendobi, Mindobi. Vide Amentom.

Mandobi assí. Grosse Eidnuss. Vide Pinhao da purgá.

II andobi do mato. Wald E. Vide Tuha de gato.

Mandobi-rana. Falsche E. IJesmorlinm axillare DC. P'apilionareane.

Manduba-rana. Vide Ararixa.

Mandubi-gnassí. Guaraniname fïr Pinhao da purgar.

Mandupava. Vide (quina do Remigio (Tuprname).

II a nu reba. Versiisster Zukerbranntwein.

Ir anga. Frucht der Mangueira.

Manga do mato. Vide Mangue do mato.

Manga da praia. Küsten M., heisst anch Abano. C'lnsia fluminensis Trian et Planch. Guttiferar. Ber. 1. 1. ph. Res., 1897, p. $2+1$. Mangaba. Frucht der Mangabeira.

Mangaba-rana. Falsche M. Hancornia speriosal valr. Girrlnerii Miill. Arg. Apocynaceap.

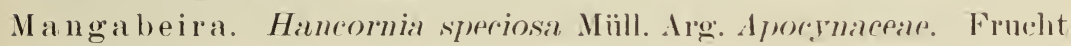
essbar.

Mangabeira in Menàs. Neriandra Martiana Müll. Arg. Aporynaceae. Caoutchouclieferant.

Mangabeira brava. Wilde M. Hancornia speciosa var. pubescens Mïll. Arg. Apocynaceae. Und Marmelleiro do campo.

Mangaiba. Vide Mangabeira.

Mangâló. Vide Angelim rosa.

Mangalô. Platycyamus Regnellii Bth. Papilionareare.

Mangalô da costa. Vide Fava de cobra.

Manganilha. Anthemis cotula L. C'ompositae.

Mangarâ. Das knollige Ende des Bliitenkolbens del Banane.

Mangarà-mirim. Vide Mangarito.

Mangarì-taia. I

Mangarà-tia. i Vide Gengibre, Tupyname.

Mangara-tyba. Vide Tinhorao.

M a ngaraz, Mangará-hy, Mangará-peuna. Caladium violareum Desf. Aracene. Hat 3 Varietäten. Arch. d. Ph., 18.5, Bd. 94, p. 263.

M angarito. Xanthosema sagittifolinm Schott. Araceae. Ph. Rdsch., 1893.

Mangarobeira. Vide Mangue de espeto. 
II angavi. Vide Comandahiba. Heisst auch Comanda-tiá.

Manga-y. Guaranibenennung der Mangaba.

Manga-ycy. Guaraniname ter Mangabeiramilch. (Harz der Mangabeira.)

Mangericao. Basilienkraut. Ocimum basilicum L. Labiatae. Kultivirt.

Mangericao de folha embaçada. Dunkelblätriges B. Virle Alfavaca do campo.

Mangericao de foll a larga. Breitblättriges B. Vide Mangericaro. Mangericao grande. Grosses B. Vide Alfavaca de cheiro.

Mangericao miudo. Kleines B. Orimum minimum L. Labiatae. Kultivirt.

Mangericao das moøas. Mädchen B. Vide Mathias.

Mangerioba und Manjerioba. Cassin sericea Swartz. Cassalpineaceae. Arch. d. Ph., 152, p. 166.

Mangerona. Majoran. Origanum majorana L. Labiatæ. Kultivirt. Mangerona do campo. Steppen M. Glechon spathnlata Bth.

Labiatae.

Mangerona grande. Vide Alfavaca.

Mangerona do mato. Wald M. Vide Serpão do mato.

Mangle. Vide Mangue verdadeira.

Mangue. Lagunucularia racemosa Gaertn. Combretaceae.

In Parà Tapura amazonica Popp. et Endl. Dichapetalaceae.

In Cearà Cassapourea macrophylla DC. Rhizophoraceae.

Mangue am a rella. Gelbe M. Avicennia nitida Iacq. Verbenaceae.

Mangue botao. Knopf M. Vide Mangue do mato.

Mangue branco. Weisse M. Vide Mangue seriba.

Mangue do brejo. Sumpf M. Gomidesia Chamissoana Bg. Myrtaceae.

Mangue canoe. Kanoen M. Bucida buceras L. Combretareae.

Mangue de espêto. Bratspies M. Stalagmites Mangle Fr. All.

Guttiferae.

Mangue manso. Milde M. Vide Mangue amarello.

Mangue do mato. Wald M. Onratea castaneafolia Engl. Ochnacer. Mangue pendao. Hängende M.

Mangue preto. Schwarze M. i Vide Mangue verdadeiro.

Mangue rasteiro. Kriechende M. Scaevola Plumeri Lam. Ciodeniaceae.

Mangue da praia, Kïsten M. Vide Manga da praia. 
Mangue sapateiro. Schuster M. Vide Mangne verdadeiro. In

Alagoas ist es Mangue de espôto.

Mangue seriba. Avicennia tomentosal lace. Verhenaceate.

Mangue vermelho. Rothe M. | Rhizophom Mallgle I. RhizoMangue verdadeiro. Nechte M. I phoraceap.

Mangueira. Mangabaum. Mangifera indiea L. Anarardiareac.

Ber. d. d. ph. Ges., 1898, p. 165.

Mangueira do mato. Vide Mangue do mato.

Ml a ng

Manguito. Manguimbá, Manico. Indianische Benennung fïl

Mangue in Parà. Vide Tapura amazonira P'. et Endl. Ber. d. d.

ph. Gies., 1901, p. 206.

Mani. Indianische Benennung des Harzes von Gulandiun.

Il aniba. In den Nordstaaten Benemnung der Mandiocra.

II a nica. Inipteracanthus visci ulus var. glomerata N. ab. E. Acanthaceate.

Maniģoba. Manihot Giasiovii Müll. Mrog. Euphorliateate. Caoutchouc-Lieferant. Arch. 1. Ph., 1.51, p. 287.

Maniçoba brava. Wilde M. Jatrophal Polliana var. mollisisima Müll. Arg. Énphorbiaceale.

Manigoba do sertao. Wüsten M. Essbare Knolle einer Enphorbiaceate.

Il a njan-gôme. Vide Maria Gomes. Indianische Benennung.

Il anjar grando. Delikate Speise. Licania deallata Hook. fil. Rosiceate.

Manimbú. S'chilfrohr zu Matten im Staate Parahyba.

Manipueira und II anicuerà. Das rom Stärkemehl getrennte, durch Kochen rom Gifte befreite Wasser der geriebenen Mandiocearï̈se.

Manipiń und Manipié. Excaecartia biglandulosil var. Klotschiana Mïll. Arg. Euphorbiaceate.

Il an iqueira. Indianische Benennung fiir Mandioceabier.

Manissoba. Blatt der Mandioccapflanze.

Maniva. Tupybenennung für Mandiocea.

Manobi. Tupyname für Amendoim.

Manoca. Haufen von Tabaksblättern zum Trocknen.

Mantimento de alraponga. Nahrung des Vogels Araponga (Chasmorhynchus nudicollis). Eugenia adstringens ('amb. Myrtaceae. 
Mantimento do pobre. Armennahrung. Virle Ilureci.

Ia io de mono. Affenhand. Vide Velame.

II á risinho. Böser Nachbar. Vide Carrapicho grande.

II a paranjúba. Baubolz. Sapotaceen.

Mapareyba. Indianische Benennung für Mangue verdadeira.

Mapichi. Mrrcia lanceolatil var. angustifolia Camb. Myrtaceate.

Mapieninga. Baum in Alagoas, liefert pfefferartige Frïchte.

Mapiguim. Brasiltabak, schwarzer, in feinen Rollen.

Mapirunga und Pirunga. Kleiner Baum in sergipe mit essbaren

Früchten. Mrrtaceae.

Mapirunga brara. Eugenial tinctorial Bgy. Mrrtalceale.

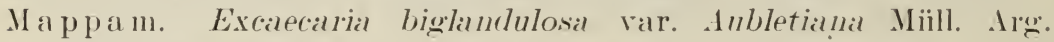

Euphorbiaceae.

Maqueira. Indianische Hängematte ron den Fasern der Tucumpalme.

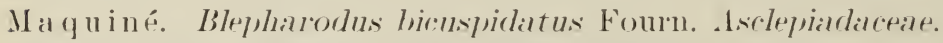

Maracutiará. Baum in Parà. Möbelholz.

Maracujá (corrumpirt von Tupyörter: maraca-Klapper, cui-iba,

- Beere). Passionsblume. P'ilssifforal alatal Klatt. Passifforacceate.

Ma racujá assu. Grosse P. Vide Maracuịa mamão.

II a racujá assì do inato. Grosse Wald P. Passifforal quadrallgularis $L$.

Il aracujá azeitona. Oliven P. Passifforal sublerosal L.

Il aracujá azeitona branea. Weisse Oliven P. I'assifforal trtamdliat Vell.

Maracujá azeitona mansa. Meisse zahme ()liven P'. Passifforal sicyoides Cham.

Maracujá branca. Weisse P. P'alssifforal rancemosal Brot.

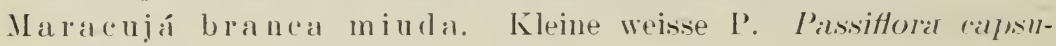
laris $\mathrm{L}$.

Maracujá cereja. Kirschen I'. Pabssiffora suberosia L. var. herteraceal Mart.

Maracujá la chapalla. Hochebenen P. Passifforat Mansoi Mart. Maracujá cobra. Schlangen P. Piassiftoral gracilis Iacq.

In St. Páulo Passifforal Eichleriania Gardn.

Maracujá coruga. Eulen P. Passiffloral cornutia Mart.

Ma racu já encarnado. Purpurrothe P. Passiffora speciosal Gardn.

II aracujá de estalo. Kinall P. Passiffora villosia Vellos.

If a racujá da estrada. Wege P. Prassiffora Vellosii (iard. 
Maracujá garapa. Kanckersaft P’. Vide Maracnjá de suspiro.

Maracujá gratude. (irosse l'. Passifforalamothystina Mart. Inch

Maracujá-assì.

Maracujá la ranjá. ()rangen I'. Pasisifforal linlifiolial 1.

Maracujá maraa. Aepfel P’. Vicle Maracujá.

Maracujá mamao. P'apaỵa P'. Passifforal milrerorallyal Mart.

Maracujá da matta. Irwald P. Passifforal solvestris Vellos.

Ma racujá melao. Melonen I’. Vicle Mararnjai cle P’arà.

Maracujá-mi. Passifforar Cormmbarensis Barb. Rodr.

M aracujá-mirim. Kleine P’. I’assifloral Walmingii Mart.

Maracujá moreega. Fledermaus P. Passifforar ranthratir Mart.

Maracujá muxilla. Vide Maracuịi de estallo.

Maracujá de pá. Holz P. Vicle Toma rocla.

Maracujá de P’arà. Passifforar maliformis Is. Passifforareane.

Maracujá perluxo. Passiffora violalreal Vellos. Passifforancearte.

Maracujá peroba. Passifforal pirodermal Barb. Rodr. Passifforalrar.

Maracujá piranga. Rothe P’. Passifforar rullar 1. Passifforalcarn.

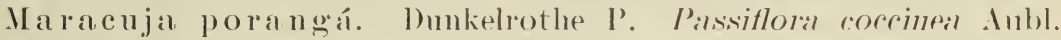

Passifforareare.

Maracujá puante. Śtinkende l'. P'assifforal fopticlal L. I'assifforalreap.

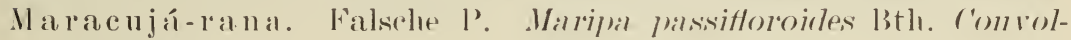
vularease.

Maracujá de rato. Ratten I'. Rasiviftoral Rarlonsile Barb. Rodr. l'assiflorarceare.

Maracujá redonda. Runde P'. Pasisifforal iodocantra Barb. Rodr. Maracujá roxa. Violette P'.

Maracujá de sapo. Frosch P'. Passifforal sylvestris Barb. lioblr.

Vellos hat auch eine $P$. silvestris.

Maracujá da serra. (iebirgs L'. Passiffora organemsis Gardn.

Maracujá desuspiro. Confekt P'. P'assiflora edlulis Sims.

Maracujá sururí. Śchmecken P. Passiflora nncronita Lanc.

Maracujá sururí assí. Grosse Schnecken P. Passiftoral arvensis Vell.

Maracujá sururíca. Sieb P'. I'asisifforal setarea 1)C.

Maracujá sylvestre. Wilde P. Vide Maracujá branca.

Maracujá de tres pernas. Dreiästige P. Passiffora-? in Bahia. Maracujá vermella. Vide Maracuja piranga.

Maraja. Astrocarinm aculeatun Wall.; in Pernambuco Bartris

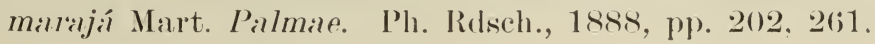


Ma rajá assì. Vide Coqueiro munbuea.

Marajá-iba. Bartris setosal Mart. Palmar. Ph. Rdsch., 1888, pp. 202, 261.

II arajá piranga. Bactris piranga Trl. P’ahmae. Ph. Rrlseh., 1888, pp. 202, 261.

Marajá rana. Bactris marajá Mart. var. Solbralensis Trl. Palnale. Ph. Rdsch., 1888, pp. 202, 261.

Marajá-y. Bactris cuspirlata Mart. var. marajá-y Barb. Rodr. Palmae. Ph. Rdsch., 1888, pp. 202, 261.

Ma ra jo ará. Curatella americana L. Dilleniaceae. Dicella bracteosa Gr. Malpighiaceae.

Marajubeira. Indianische Benennung eines Baumes, welcher zur Anfertigung von Canoen benutzt wird.

Maraka. Vide Cascavel. (Maraka Tupywort fïr Klapper.)

Maranacaba und Marangaba. Vide Coqueiro Yatai.

Maranha grande und Maranịa grande. Vide Maracujá assì. M a rapaub. Bäume in Parà. Möbelholz.

Marapuama. Liriosma ovata Miers. Olarinarceale. Ber. (l. d. ph. Ges., 1901, p. 41.

II a raquitaia. Bauholz in Parì.

Maratarì. Vide Capim de Angola.

Maratataba und Marataua. Vide Tataiba.

Maravaia. Vide Capim camellão.

Maravilha. Wunderblume. Mirabilis dichotoma L. Nyctaginaceae. Ph. Arch., 1897.

Maravilha do campo. Steppenwunderblume. Vide Capipoatinga.

Ber. d. d. ph. Ges., 1901, p. 211.

II a r can a hy ba. Cassia multijuga Rich. Caesalpineaceae.

Marcella. Volkcolruption von Macella.

Marco Gonzalo. Bauholz in Parà.

M arfim de follh a grande. Grossblattriges Elfenbeinholz. Melochia umbellata Fr. All. Sterculiaceae. Ber. d. d. ph. Ges., 1900, p. 60. I arfim falsa. Falsches Elfenbeinholz. Melochia hirsuta Cav. Sterculiaceae. Ber. d. d. ph. Ges., 1900, p. 60.

Marfim vegetal. Vegetabilischen Elfenbein. Phytelephas macrocarpa R. et P. Palmae. Ph. Rdsch., 189:3.

Margaraz. Caladium violaceum Sch. Araceae. Arch. d. Ph., 144, p. 26.5. 


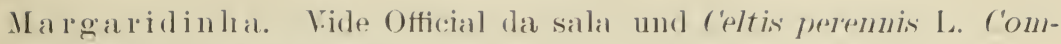
positale. Gartenpflanze.

Ma ri. Geoffioral superlua HBKth. Papplionaceace.

II a ri-m a ri. Vide Camna fistula. In den Noldstanten Cassibil leianrlral Bth. Caesilpineareare.

Maria branca. Weisse Maria. Viule Claro d'ovo. Heisst auch (iosmenta.

Maria catingente. Unangenehm riechende M. M.vroiaria mirrantha Bg. Mrrthaceae. Aeolanthus suavis Mart. Lahiatae.

Maria cheirosa. Wohlriechende M. Melosanthus longespricatus

Pohl. Lalbiatale.

Ma ria congo. Negerin Maria. Ascleplias rnlassavir.y L. Asclepiadareale. Virle auch Carurí bravo.

Maria da costa. Vide Herva da costa.

Maria (iomes. Visle ()ra pro nobis mindo.

Maria gorda. Fette M. Ocimmm rarnosmm Link et Otto. Lalliatir. II aria leite. Milehgebende M. Vide Herva de Santa Luzia.

II r ria molle. Weiche M. Virle Umbì.

Maria nica. Kleine M. Vicle Capericoba.

Maria Pereira. Birnbaum M. Posoqueria macropus Mart. Rnhiacease.

Ma ria preta. Schwarze M. Blanchetial heterotrirha I)('. Compositir. Conoclidinm masibolinm DC. C'ompositae. Ferner vide Mossetaiba, Barahuna und Marmeleira da terra. In Pernambuco Vitex polarg*ima Blanch. Terbenacere.

Maria preta de campina. Vide P’á de cavallo.

Maria preta do mato. Mald M. Eupatorium ballotaefolium HBKth. Compositue.

Maria Rosa. Cocos Procopiana Dr. Palmae. Ph. Rdsch., 1888.

II a rianeira. Acnistus cauliflorus Schott. Solanaceae. Arch. d. Ph., 141 , p. $240 ; 143$, p. 119.

Marianinha. Kleine M. Commelina deficiens Hook. In Menas Commelina elegans Humb. Commelinareae. Ph. Rdsch., 1892, p. 2.58 .

II a rianinha de folha la rga. Vide Marinheiro de folha larga. Maricá. Vide Unha de gato.

Maricá assì. Grosser Bauch. Canarillesia arborea K. Sch. Bombaceae. Ber. d. d. ph. Ges., 1900, p. 170.

II arinheiro. Matrosenpflanze. Benennung für Assafrôa, Gito, in s. Paulo für Macaqueiro. 
Ir a riulueiro do campo. Steppen M. Trichelial cathartical Mart.

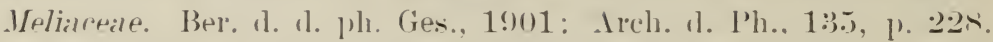

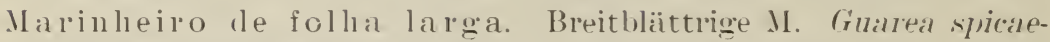
Horal d. Tuss. Melialceale. Ber, d. d. ph. Ges.. 1901: Areh. d. Ph., 1:35. p. 2.2.2x.

Ir a rinheiro de folha miuda. Kleinbliittrige Ir. Trichelia emar-

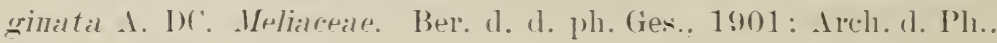
13.5. 1. 2.2s.

Il aripá. Maximiliana Marifua Mart. Palmale. Ph. Radseh., 18st), 1. $1: 34$.

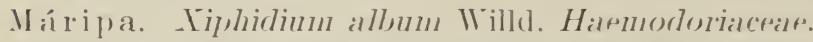

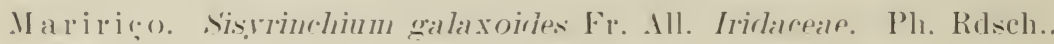
$18: 1 \cdot 2,1) .13 \cdot 2$.

Il aripico braro und M. syluestre. Wilde M. Hipoxis deramhetis L. Hypoxidaceap. Areh. d. Ph.. 160, p. 1335.

If aripico do mato. Wald M. Hrpoxis decumbens L. var. maior. sent. Hrpoxirlaceale. Areh. 1. Ph.. 160. p. 135.5.

If armajuda brara. Wilde M. Vỉe Lrucú braro. Ber. d. d. pl. (ies.. 1890) p. Tt.

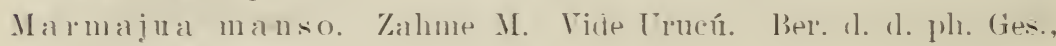
$1 \times(s)(1), 74$.

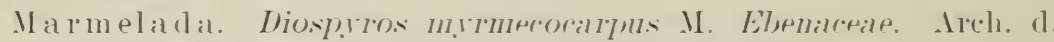
Plı., 160). P. 136.

If armeleiro. (quittenbaum. Crdonial rulganis Pers. Rosalceale. Kultivirt.

Ma rmeleiro (do Brazil). Viale Marmelatla.

In Alagoas Dirhapetalum orloratum Baetl. Dirhalpetalareate.

In Rio (irande do sul Guidonia ulnifolial ('amb. Flarourtiareate.

In Rio de Janeiro vide Maria preta do mato.

Il a rueleiro do campo. (quittenbaum der steppe. und II armello

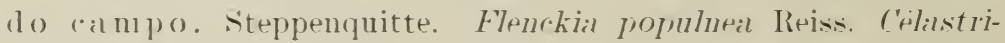
niaceale.

If armeleiro de cheiro. Wohlriechencle Q I Vide (quelua faras. Marmeleiro do mato. Wilder (2. Casearial Cambesserlesii Engl.

Flacourtiaceate. Ber. d. 1. ph. (ies., 1899, p. 172.

In ameleiro dosertao. Wiisten Q. Phrllanthus graveolens Miill.

Arg. Euphorbiaceare.

Il armeleiro da terra. Einheinischer (2. Vide Marmelada. 
Il amelinho. Kleiner (quittenbaum. Tournefortial laverigatal Lam.

Ineliotropaceale. Arch. I. Ph., 1(i), ). 1:3(;.

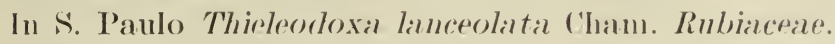

Il armelinho do campo. KI. (2. (ler Steppe. Maprounral hrasiliensis St. Hil. Enpliorliacear.

In S. Paulo Alibertia ronrolor Schum.; Alihertial sessilis sichum. línbiaceare.

Marolinho. Aberemoil tioica Barb. Rodr. Anonicrale.

II arolinho do campo. Anonal relalensis Barb. Rodr. Anonareale. II orôlo und M. an arello. (ielbe M. Anona Rodrignesii Rarb. Rodr.

Anonarear. Ber. d. d. ph. (ies.. 1s:)7, p. 4 (i9.

Io öoró. Vide Jarí.

Ilaroio. Andorn. Myptis spirata Pois.; Leonnrus sibirimes L. Lilliatile.

Marroio do nato. Vide Herva cirleira do mato.

Martello. Vide Rasteiro.

II aruaruna. Indianische Benennung für Ilgodoeira brava. IBer. d. d. ph. Gies., 1900, p. 421 .

Marubá und Marupá. Simaruba amaral Aubl. var. oparaa Englel.

Simaruharene. Ber. (1. (1. plı. Ges., 189), pp. 4:31 1. 429), 4:37.

Marubá mirim. Kleine M. Simaba glandnlifera Gard. Simarn-

biaceale. Ber. d. d. ph. Ges., 1898, pp. 4:31 11. 429, 4:37.

Marúpa und Marupa-y. Marnpa Franroana Miers. Simarularrear.

Ber. d. d. ph. Ges., 1898, pl. 4:31, 11. 429). 4:37.

Marupa-hy. Vicle Gito.

Marupaúba. Bignoniareare. Heilmittel.

Marupeúba. Eriocaulon Kunthii Koke. Erioranlareare.

Marupíeba. Vide Cajazeiro do mato. Ber. d. d. ph. Ges., 1898, 1. 1.58 .

Ma ry roba. C'oros malcrocarlyil Barb. Rodr. Palmae.

M assambarí. Sorghum halepense Pers. Gramineale. Plı. Ridsch., N. Y., 1894 .

In Matto Grosso Desmoncus ruyahensis Barb. Rodr. Palmate.

II assa randiba. Eugenia Mrignifica Sp. Myrtaceae.

II assaranduba. Mimmsops alata Fr. All. Sapotaceate.

In S. Paulo Chrysophyllum ebenaceum Mart. Sapotaceae.

In Parà Chrysophyllum brasiliense A. DC. Sapotaceae.

Massaranduba assì. Grosse M. Minnusops excelsal. Fr. All. Sápotaceae. 
Massarancluba branca. Weisse M. Lucumia procera Mart. Salpotirrare. Ph. Rulsch., N. Y., 1§s8, p. 7 ; \%eitsch. d. oest. Ap.-Ver., $186(;, p .: 3+7$.

Uassaranduba de leite. Milch M. Mimmsops Horibmola Mart. Silpotilceile.

Massaldanduba das taboleiras. Hochebenen M. Mimmsops triffora Fre. All. Silpotaceale.

Massaranduba vermelha. Rothe M. Chrysophyllum obtusifolinm

Fr. All. Silpotaceare.

Massaracuri. Palme am Amazomenstrom. Palmae.

Mastruco. Kresse. Semmebiera pinnatificla D)C. Crnciferae. Areh. d. Ph., 110, p. 1:36; Ber. 1. d. ph. (ies., 1897, p. 2хт.

In Bahia vide Herra de Combrigueira.

In Parai vide Agrião le P’ài. Areh. 1. Ph., 116, p. 22.27.

In Rio Cirande do Sul Sennelicral serratal Poir. Finciferace

Mastruço d’agua. Wasserkresse. I IMymaria cordatal Willd. Mastruco do brejo. Sumpfiresse. I ('aryoplirllaceate. Ber. d. d. pl. (ies., 1!)1, p. 211.

Mastruco da terra. Einheimische K. Lapirlinm ruderale L. C'rucifirite.

Mataba-iba. Matallal gunianensis Aubl. Silpindaceane.

II a tabaratas. Schabentödter. Andiral —-" I'alpilionarrale.

II a ta cablas. Ziegentiolter. Ipomoeal roseal Chois. Convolvulacear.

In Parì vide Algodausinho.

- Mata cachorro. Hundetödter. Bernardinia Hnminemsis Panch.;

Romreal glabral HBKth.; C'ommarus cymosus Planch. 11. C'. Ileanus Gilg. C'onnaraceate. Ph. Areh., 1901.

Borjonial racemosil Don. Asrlepladalceale.

II ata cães. Hiindetölt r. Vide Angelica de rama.

Mata calada. Schneetöltend. Wurzel einer Mandiocea ähnlichen Enyliorlincear.

Il at a canna. Kuckerrohrtödter. Virle Caa-ataya.

In Bahia Lindernia riffissal Wettst. Sorophulariaceae.

II a a fome. Hungerstillend. Benemung einer Mandiocca Varietat. Il at a fome brava. Wilder H. Serjanial rommmmis Radl. und $S$. lethalis St. Hil. Śaplinklarepar.

Il a ta-mata. Schildkrötenbaum. Lecothis roriacea D) Myrtarease. Arch. (l. Ph., 102, p. 16;.

I a ta ollios. Blinchmachender Banm. Opthalnopliston pedunculare Miill. Arg. Enphorbiaceae. 
Mata olhos manso. Milder B. Lucuma nereifolia Hook. et Arn. Silpotaceale. I'h. Rdsch., N. Y., 1888 , p. 3:3.

Mata paó. Baumtödter. Struthantus orbicularis Bhum. Loranthaceae. Ph. Arch., :3, p. $1+1$.

In den Nordstaaten Renggeria comams Meiss. Guttifeme. Ber. d. 1. ph. Ges., 1897, pp. 241, 242. Virle $\Lambda$ puy.

Ir ta paó de espinha. Stachliger B. Ceiba Riviera Schum. Bombaceae. Ber. 1. d. ph. Ges., 1900, p. 161.

Mata pasto. Weidentödter. Cassial retienlatal Willd., ('. alatal L. und $C$. bicapsularis L. l'alesalpineafeate. Vide auch Mangerioba. Mato pasto vermelho. Rother IV. Cassial stipulatal ('arsalpineacèae.

Matapi. Fischreuse von Taquararohr ler Indianer.

Mata-rana und. Matata-rana. Costus lanceolatus C. G. Petersen. Zingiberaceas.

Matata ub. Vide Sambacium.

Mate. Nationalthee Südamerikas. Ilex paraguarensis st. Hil. Aquifoliaceae. Arch. d. Ph., 82, p. 951.

In Minas Ilex domestica Reiss. Ztsch. d. ist. Ap.-Ver., 1881, p. 257. In s. Paulo Ilex sorbilis Mart.

In Rio de Janeiro Ilex theezans Mart. Virle auch Congonha.

Mate bravo. Wilder M. Ilex affinis Gard.

Mate do campo. Steppen M. Luxemburgia polyandra St. Hil. Ochnaceare. Vide Chá de bugre.

I ate me embora. Calystegia sepinm $R$. Br. var. maritima Chois. Convolvilaceae.

Mathias. Moquinial flavescens (Aard. C'ompositar.

M a tico. Vide Herva de soldado.

M a tiri. Indianische Ienenmung des Presssackes der Tucumfaser zur Pressung der geriebenen Mandiocca.

Maturi. Der unreife Kern der Cajúfrucht zu Confekt.

Máu visinho. Böser Nachbar. Kanthoxylum stelligerum Engl. Rutaceale Ber. d. d. ph. (ies., 18:)!), p. :34(;.

Má́ba branca, M. preta und M. da varzea. Bauhölzer.

Ma úba do mato. l'ommarus norlosms K. l'ommaracear.

Mà̀e molle. Weicher M. Vide (otficial da sala.

Maxixe. Vicle Machicha.

II axixenho. Volksmittel in Mlagoas.

Ma yaca. Xyris pallicla Mart. Kyridaceate. Ph. Redsch., 18!)2, p. 164. 
J beerú, Beery und Birú. C'anna elulis Ker. Cannareale. P'lr. Rdsch., 1893, p. $25 \%$.

Mea pé. Tuprbenennung für Brod ron Mandioccamehl.

Medicineiro. Arzneinuss. Jatropha elliptica Mïll. Iro. Enphorbiarreate.

Meimendro branco. Weisses Bilsenkrant. Hroscramms allums L. Solanaraze. Kultirirt.

Meimendro preto. Schwarzes B. Hyosrarmms niger L. Solinnarer. Kultivirt.

Meín. Meír preto, Meirú preto, Meum preto. Gruatterial macropus Mart. Anonaceale. Ber. d. d. ph. Ges., 18!)T. p. 4(j:3.

Meladinlia. Honigpflanze. Stemodia thrantifolia Niw: Scroplunlariaceae. Vide auch Paracary.

Meladinha falsa. Falsche H. Mipteraranthns jorrigens Nab. E. Acanthareare.

Melado. sirrup des Zuckerrohrs.

Ifelambo und Il alambo. Irims's winteri Forst. var. granademsis Eichl. W"̈ntheraceare. Ber. d. d. ph. Ges., 1s96, p. 161.

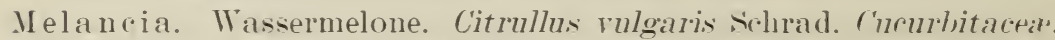
Melaneia do campo. Steppen II. Melancimm rampes"re Naur. C'ucurlitaceale.

Melancia de cobra. Śrhlangen IV. S̈̈ros quinquelobatus Cogn. l'ueurbitaceate.

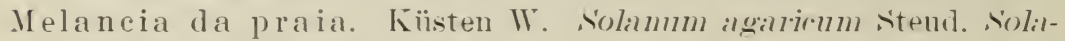
naceae.

If elar. Melone. r'ncmmis melo L. Cucurbitactare.

Melao de beiga flor. Kolibri M. Vide (ereja da purara (Melotria). Ifelao de caboclo. Indianermelone. Viđle Crôa.

Ielao de si. Caetano und M. de s. Vincente. Momorliria rharantia L. Cincurbitareate. Arch. d. Ph., 94. p. :3(;3).

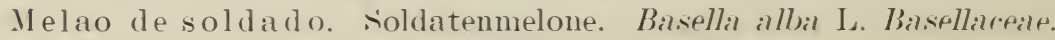
Kultirirt.

Melindro und ('abello de moca. Liliacere. ('artenpflanze.

Ifella pinto. Stellaria media Vill. Alsinaceate.

Mello pinto. Vide Herva tostão.

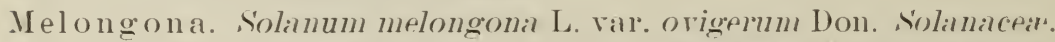
Melosa. Kleber. Wissidula periplocifolia Prsl. Malvacear. Rer. 1. d. ph. Gies.. 1900, p. 267 .

In C'earà Ruellia asperulil Lind. Acinthaceale. 
Membui-axio. Rohrflöte der Indianer. (Von Membú-Flöte, iaxo -weinen.)

Mendanha. Abutilon inaequale K. Schum. Malvaceae. Ber. d. d. ph. Ges., 1900, 1. 266.

In Espirito Santo und Rio vide Tres follhas vermelhas.

In S. Paulo vide Cuaiarana.

In Minas Schweiggeria florilunnda St. Hil. Violaceae. Ber. d. d. ph. Ges., 1897. p. 99.

Mendobim. Vide Amendoim.

Me nd ocá. Taligalial campestris Aubl, val. puniceal Brig. Verhenacea. Menjuba. Bauholz.

Mentrasto. Vide Herva de S. Joaõ commun.

Mentrusto. Vide Mastruço.

Mentruz. In Bahia für Herva de lombrigneira.

Mercurio dos pobres. Quecksilber ler Armen. Vicle Manacâ.

Mercurio vegetal. Vegetabilisches (quecksilber. Viđle Manacâ.

Mercurio do campo. Steppen (i. Vide Gallinha choca.

Merendiba und Mirindiba. Terminalia Ianuarieusis DC. und $T$.

Riedelii Eichl. Combretaceae.

In Cearà vide Amendoeira brava.

In Pernambuco Buchenavia rapitata Eichl. Combretaceae.

Merendiba bagre. Terminalia Riedelii Eichl. C'ombretaceae.

Merendiba rosa. Lafoensia glyptocanpa Koehn. Rosaceae.

$\left.\begin{array}{l}\text { Merendiba roxa. } \\ \text { Merendiba tinta. }\end{array}\right\}$ Terminalia phaeocarpa Eichl. Combretacex.

Meri, Miri und Miró. Dipholis salicifolia A. DC. Sapotaceae.

Merú, Мurú und Mecrú. Vide Albarí. Ph. Rdsch., 1893, p. 257.

Merù a und Merú-caã. Spermacoce longifolia Lacerd. Rubiaceae.

Meruana, richtiger Merú-rana. Falsche Merú. Cnestidium lasio-

carpum Bak. Connaraceae.

Métará. Runde Holzscheibe als Lippenschmuck der Indianer.

Mexerica. Leucothoe breviflora Meissn. Ericaceae.

Mexerico. Staphidium pauciflorum var. stellatum Naud. Melastomaceae.

Mexerico branco. Weisse M. Leandra scabra DC. Melastomacea. Mijo de cavallo. Pferdeharn. Agaricus cretacens Agaricaceae.

Mil homen. Tausendmännerkraut. Aristolochia cymbifera M. var. cymbifer: Mart. Aristolochiaceae. Ph. Rdsch., 1893, p. 182; Arch. d. Ph., 82, p. 92. 
In Rio Grande do Sul Aristolochia triangularis M. Aristolochiaceæ.

Ph. Rdsch., 1893, p. 182; Arch. d. Ph., 82, p. 92.

Mil homen arvore. Tausendmännerbaum. Sclerolobum aureum Bth. Papilionaceae.

Mil homen miudo. Kleines T. Vide Jarrinha.

Mil em rama. Schafgarbe. Achillea millefolium L. Compositae. Kultivirt.

Milho. Mais. Zea Mais L. Gramineae. Arch. d. Ph., 91, p. 107; Ph. Rdsch., 1894, p. 165.

Milho d'agua. Wassermais. Victoria Cruziana d'Orbg. Nymphaeaceae. Ber. d. d. ph. Ges., 1897, p. 284.

Milho d'Angola. Negermais. Sorghum vulgare Pers. Gramineae.

Milho bravo. Wilder M. Tripsacum dactyloides L. var. genuinum W. Gramineae.

Milho cozido. Gekochter M. Licania incana Aubl. Rosaceae.

Milho do mato. Wald M. Vide Massambarà.

Miloló. Anona reticulata L. Anonaceae. Ber. d. d. ph. Ges., 1897, p. 458.

Mimo de Venus. Hibiscus Rosa sinensis L. Malvaceae. Ber. d. d. ph. Ges., 1900, p. 271.

Mimo de Venus da terra. Einheimische. Goldfussia anisophylla Nees. Acanthaceae.

Mindobi-guassú. Grosse Erdnuss. Vide Mundubi-guassú.

Mindobi-rana. Falsche Erdnuss. Vide Ararixa. Ber. d. d. ph. Ges., 1900, p. 52.

Mindobi bravo. Wilde E. Vide Raiz de corvo.

Minerva. Lagerstroemia indica L. Rosaceae. Kultivirt.

Mingú. Vorzügliches Bauholz in den Nordstaaten, es existiren 3 Varietäten: pardo-braunes, preto-schwarzes, roxa - violettrothes Holz.

Minnána. Oenothera indecora St. Hil. Onagraceae.

Mió-mió. Baccharis cordifolia DC. Compositae.

Miracem. Solanum? Solanaceae.

Mira-itan. Vide Marapuama.

Mirichy und Miriti. Vide Buriti.

Miroró. Vide Unha piquena de vacca.

Mium. Urostigma euomphalum Miq. Urticaceae.

Miúva. Vide Aninga pari.

Mixira. Conserve von Fisch oder Fleisch in Schildkrötenfett. 
Mocajá. Vide Coqueiro de catarho.

Mocitayba. Vide Mossetaiba.

Moco branco. Bauholz in den Nordstaaten.

Mocohim-caea. Vide Mokokim-kaka.

Mocororó. In Cearì der gegohrene.Cajúsaft; in den anderen Nordstaaten ein erfrischendes Getränk verschiedener Fruchtsäfte.

Mocoto. Elythraria tridentata var. caulescens Vahl. Acanthaceae. $\mathrm{Mocub}$-ass ù. Bauholz.

Mocury. Essbare Frucht im Staate Pernambuco.

Mof ungó. Chamissoal altissima HBKth. Amarantaceae.

Mohica. Guaranibenennung für Buranhem.

Mojica. Speise. Brei von Mandioceastärkemehl und Fleischbrïhe. Moira-pinima. Vide Mnira-pinima.

Moliana. Vide Colher de vaqueiro.

Moluca. Vide Buranhem.

Moluri. Aegiphilia obducta Vellos. Verbenaceae. Heisst auch Fructo de papagaio.

Mombaca-xió. Rohrflöte der Indianer.

Mondé und Mundé. Croton palanostigma Kltsch. Euphorbiaceae. Mondúcurú und Mandacarú. Heilpflanze.

Monguba. Frucht. \Bombax mungaba Mart. etZuce. Bombacex. Mongubeira. Baum. $\}$ Tupy von Mong-Klebestoff, yba-Baum. Ber. d. d. ph. Ges., 1900, p. 165.

Monjólo. Stampfmühlenholz. ～Pithecolobium monjolo Mart. Monjólo vermelho. Rothes St. $\}$ Mimosaceae.

Monjólo de ferro. Eisen St. Pithecolobium rhombeum Bth. Mimosaceae.

Monsenhor amarello. Pyrethrum Parthenium L. Compositae. Gartenpflanze.

Moquéca. Geröstete Speise mit Capsicum.

Morango. Erdbeere. Fragaria vesca L. Rosaceae. Kultivirt.

Morango do campo. Steppen Erdbeere. Ephedra americana Willd.

Gnetaceae.

Morcegueira. Fledermausbaum. Andira inermis HBKth. Papilionaceae.

Moringúa. Vide Milho d'agua.

Morixó. Vide Marajoará (Dicella).

Mororó. Vide Cipo de escada, Jasmin do mato und Unha de vacca. Mororó branco. Sloania monosperma Vellos. Tiliaceae. Ber. d. d. ph. Ges., 1898, p. 286. 
Mororó do sertao. Bauhinia flexuosa Morio. Caesalpineaceae.

Morototó. Didymopanax Morototoni Don. et Planch. Araliaceae Morototó bravo. Wilder M. Didymopanax mimosum E. March. Araliaceae.

Mosquiteiro. Vide Sete casacas.

Mossetaiba. Zollernia falcata Nees. Cilesillpineaceae.

Mossetaiba do campo. Zollernia ilicifolia Vog. Caesalpineaceae. Mossútaiba. Swartzia crocea Bth. Papilionaceae.

Mozes. Mimosaceae. Bauholz. Asche reich an Kali.

Moteté. Cucurbitaceae. Heilpflanze in Alagoas.

M u cambá. Verbesina glabrata Hoak. et Arn. Compositae.

M uca rá-caa. Nierembergia graveolens St. Hil. Solanaceae.

Mucátaia und Mucútaia. Nectandra incanescens Nees. Lauracex,

Ph. Rev., 1897.

Mucauba und Macauba. Acromia sclerocarpa Mart. Palmae.

Muсuси́ merim. Kleine M. Aulomyrcia amazonica Bg. Myrtaceae. М и си́ һ und М оси́i. Urwaldbaum in Alagoas mit essbaren Früchten. M uсuјá. Acromia sclerocarpa Mart. var. Wallaciana Dr. Palmae. Mucujé. Vide Macujá.

Mucúna. ， Mucuna pruriens DC. Papilionaceae. Vide Mucunã (Mucunan). $\}$ auch Olho de boi und Pós de mico.

In Matto Grosso Mucuna Matto Grossensis Barb. Rodr. Papilionaceae.

In Minas Dioclea lasiocarpa Mart. Papilionaceae.

Mucúna branca. Weisse M. Solanum asterophorum Mart. Solanaceae.

Mucúna do mato. Wilde M. Dolichos rostrata Bth. Papilionacex. Mucúna uassú. Grosse M. Vide Coró-onha.

Mucunã in Parà. Dioclea lasiocarpa Mart. Papilionaceae.

Mucungá und Mangunvá. Indianische Benennung für Canjica.

Mucurá. Vide Pipi verdadeiro.

Mucurá-caa. Vide Mucará-caa. In Tupy bedeutet Blatt von Gamba (Didelphus) Geruch.

Mucuyba. Baum mit Oel liefernden Samen in Alagoas.

II ufumbá. Combretum leprosum Mart. Combretaceae.

Mufumbó und Mofumbó da beira rio. Crotalaria anagroides

HBKth. Papilionaceae.

Muhi. Orophoma Carana Spruc. Palmae. Ph. Rdsch., 1888, p. 134, Muira. Vide Muyra. Tupibenennung für Holz etc. 
Muivá. Vide Aninga-pari.

Mulungú und Murungú. Erythrina mulungu Mart. Papilionaceæ. Arch. d. Ph., 102, p. 168.

In Minas Erythrina cristagalli L. Papilionaceae.

Munbaca und Munboca. Astrocaryum munbaca Mart. Palmae. Ph. Rdsch., 1888, p. 261.

Mundahú. Phyllanthus nobilis var. brasiliensis Müll. Arg. Euphorbiaceae.

Mundé. In Tupy = Farbe. Croton palanostigma Kl. Euphorbiaceæ. Mundubi. Vide Amendoim.

In Parà Cassia diphylla L. Caesalpineaceae.

Mundubi-rana. Vide Ararixa. Ber. d. d. ph. Ges., 1900, p. 52.

Munduy-guassú. Vide Pinhão da purga. Arch. d. Ph., 102, p. 160.

Mungabeira. Eriodendron speciosum Fr. Allem. Bombaceae.

Mungúbeira. Vide Mongabeira.

Munipúi. Vide Manipiú.

Murapicá und Murupicá. Sebastiania corniculata var. oligophylla Müll. Arg. Euphorbiaceae.

Múra-piranga. Rothe M. Haploclathra paniculata Bth. Guttiferae. Ber. d. d. ph. Ges., 1897, p. 229.

Murareina. Vide Rabo de ararà.

Murcella. Vide Rabo de porco.

Mureci, Murici, Murusi. Byrsonima verbascifolia Rich. Malpighiaceae. Früchte essbar'; in Cearà wird eine Speise davon bereitet, Cambica benannt.

In Parà Byrsonima cinerea DC. und B. crassifolia Kth., B. spicata Rich.

In Pernambuco Byrsonima nervosa DC.

M u reci a ma rello. Gelbe M. Cassia verrucosa Vellos. Caesalpineacere. Mureci cascudo. Dickschalige M.

Mureci guassú. Grosse M.

\} Vide Mureci.

Mureci de fructo miudo. Kleinfrüchtige M. Byrsonima sericea DC. Malpighiaceae.

Mureci de lenha. Brennholz M. Byrsonima verbascifolia Rich. var. leiocarpa Grieseb. Malpighiaceae.

Mureci penima und M. pinima. Gefärbte M. Byrsonima chrysophylla Rich. Malpighiaceae.

Mureci penima cipo. Gefärbte M. Liane. Heteropteris chrysophylla Kth. Malpighiaceae. 
Mureci petinga und pitinga. Brrsonima crassifolia Kth. Malpighiaceae.

Mureci da praia. Küsten M. Bırsonima arbutifolia Gr. Malpighiaceae.

Mureci da serra. Gebirgs M. Vochrsia Goeldii Hub. Voch rsiaceae.

Mureci dos taboleiras. Hochebenen M. Vide Mureci da lenha.

Muriti. Vide Buriti.

Murriao. Anagallis arrensis L. Primulaceae. Kultivirt.

Murta. Mrrte. Eugenia lucida Camb. Mrrtaceae.

In Parà Mouriria guianensis Aubl. Melastomaceae.

Murta branca. Weisse If. Campomanesia desertorum Bg. Myrtaceae.

If urta de cheiro. Wohlriechende II. Vide Jasmin laranja.

Murta do mato. Wilde M. Tide Quina de Piauhy.

If r rta pelluda. Zottige M. Mrrcia bracteata DC. Mrrtaceae.

Murta preta. Schwarze M. Aulomsrcia nigrescens Bg. Mrrtaceae.

Mu ra do reino. Europäische M. Mrrtus communis L. Mrrtaceae.

Kultivirt.

Murta vermelha. Rothe M. Eugenia macrosperma Dr. Mrrtaceae.

Murtilha. Kleine M. Mrrhinum rubriflorum Bg. Mrrtaceae.

Mlurtilla und Murtinha. Blaubeeren M. Mrrciaria disticha rar.

Bahiensis Bg. Mrrtaceae.

Murtinha. Kleine Blaubeeren M. Mrrrhinum atropurpureum Schott. Mrrtaceae.

Murtinho. Kleiner Mrrtenstrauch. Eugenia Candolleanum DC., E. oralifolia Camb. Mrrtaceae.

Murtinho carquejá. Bitterer M. Blepharocalrx amarus Bg. Mrrtaceae.

Murtinho manso. Milder M. Iossinia lucida DC. Mrrtaceae.

Murtinho da praia. Küsten M. Eugenia arenaria Camb., E. insipida Camb. Myrtaceae.

M urá. Vide Caeté.

Murucaia rana. Falsche Passionsblume. Vide Maracujá-rana.

Muruch g. Tide Buriti.

M urucú. Holzlanze mit Curari vergifteter Spitze der Murú-Indianer.

M u rugem und Morugem. Stellaria media Vellos. Alsinaceae. Eingeführt. 
Murugem bastardo. Vide Murrião.

M urumurú. Astrocaryum mirumuru Mart. Palmae. Ph. Rdsch., 1888, pp. 262-265.

Murumurú-iry. Astrocaryum farinosum Barb. Rodr. Palmae.

Ph. Rdsch., 1888, pp. 262-265.

Murumurú de Iavary. Astrocaryum Iavarense Trl. Palmae. Ph.

Rdsch., 1888, pp. 262-265.

Murumurú mirim. Kleine M. Astrocaryum minus Trl. Palmae.

Plı. Rdsch., 1888, pp. 262-265.

Murumurú da terra firme. Festland M. Astrocaryum minus

Trl. var. terra firme Dr. Palmae. Ph. Rdsch., 1888, pp. 262-265.

M u r u ré und M ur ur ù. Bichetial officinalis Heerm.?; Urostigma cysto-

podum Miq.? Urticaceae. Ph. Rdsch., 1891, p. 167; Areh. d. Ph.,

102, p. 167. Vide auch Gigogo. Ber. d. d. ph. Ges., 1897, p. 285.)

Mururé d'agua. Wasser M. Vide Apé.

Mururé-rana. Falsche M. Vide Coré bravo.

Mururé-yg. Wasserspeise. Eichhornia aznrea Kth. var. rhizantha

Seub. Pontederiaceae. Ber. d. d. ph. Ges., 1901, p. 95.

Murussú-garana. Tupyname für Maracuịa-rana.

Murutá-caa. Tupyname für Paó amarello.

M usgo de café. Kaffeemoos. Parmelia appendiculata Mart. Lichenes.

Mussambé. Dactylaena micrantha Schrad. Capparidaceae. Ber. d.

d. ph. Ges., 1898, p. 41.

Mussambé cheiroso. Wohlriechende M.

$\left.\begin{array}{l}\text { Mussambé branco. Weisser M. } \\ \text { Mussambé bravo. Wilder M. }\end{array}\right\}$ Vide Sete Marias.

Mussambé de espinha. Vide Bredo fedorento.

Mussa mbé de sete folhas. Siebenblättriger M. Vide Sete folhas.

Mussambé da serra. Gebirgs M. | Cleome rosea Vahl. Cappari-

Mussambé vermelho. Rother M. $\}$ daceae. Ber. d. d. ph. Ges., 1898, p. 43.

Mussotaiba. Vide Mossetaiba.

Mutá-mutá cipó. Tupyname für Cipó de escada.

Mutambá, Matombá, Motombá. Guazuma ulmifolia Lam.

Sterculiaceae. Ber. d. d. ph. Ges., 1900, p. 120.

Mutambá peretiqueino. Vide Corindiba. Ph. Rdsch., 1892, p. 35.

Mutambá preta. Schwarze M. Mollia speciosa Mart. et Zucc.

Tiliaceae. Ber. d. d. ph. Ges., 1898. p. 284.

Mutúm ujú. Vide Arâriba und Putí-mujú. 
Muyra-coatiará. Gefärbtes Holz. Centrolobium robustum var. microchaete Mart. Papilionaceae.

$\left.\begin{array}{l}\text { Muyra-hobi. } \\ \text { Muyra-ita. }\end{array}\right\}$ Steinholz. Vide Paó ferro.

Muyra-ketyca. Pfefferpflanze. Tupyname für Cipo de carijó (Davilla).

M u yra-pajé. Arztbaum. Tupyname für Cumarú.

M и y ra-pin ima. Gefärbter Baum. Coussapoa nitida Miq. Urticacex. Muyra-piranga. Rothes Holz. Tupyname für Paó Brasil.

Muyra-puama. Vide Marapuama.

Muyra-tan. Festes Holz.

Muyra-una. Schwarzes Holz. $\}$ Vide Barahuna.

Najá. Vide Anajá.

Najarana. Byrsonima spicata Rich. Malpighiaceae.

Nambú. In Pernambuco eine kultivirte Dioscorea-Art.

Namoym. Baum in Parì. Die gekochten Früchte Nahrungsmittel der Indianer.

Naná. Guaranibenennung für Ananas.

Nana-y. Ananaswein.

Nancibea. Manettia cordifolia Mart. Rubiaceae.

Nandiroba. Vide Andiroba seu Iabotá. Tupy Nandy-Oel, yroba - bitter.

Nani und Mani. Indianische Benennung des Harzes von Oanani.

Natal und Flor de Natal. Weihnachtsblume. Vide Manacã.

Navalla de macaco. Affenrasirmesser. Vide Capim navalha.

Navalheira dura. Scharfe Muschelschale. Hypolytum rigens

N. ab. E. Cyperaceae.

Navalheira molle. Weiche M. ) Hypolytum laxum Schrad. Navalheira miuda. Kleine M. $\}$ Cyperaceae.

Negreira. Corrumpirt von Negrurá. Schwärzlich. Iussiaea Louroutheuna Camb. Onagraceae.

Negra mina. Auch Catinga de negra. Negeringeruch. Citriosma oligandra Tul. Monimiaceae. Ber. d. d. ph. Ges., 1896, p. 93.

$\mathrm{Nhá}$ und $\mathrm{Nia}$. Vide Castanheira de Maranhaô.

Nhaguarandi. Pilocarpus Jaborandi Holms. Rutaceae. Ber. d. d. ph. Ges., 1899, p. 335.

Nhambi. Ottania Warabacoura Miq. Piperaceae. Ph. Rdsch., 1894, p. 287. 
Nhambú, Neambú, Nia mb ú. Spilanthes acmella L. var. oleracea Compositae.

Nhambú guassí. Grosse N. Indianische Benennung für Mamona (Ricinus).

Nhambú-rana. Falsche N. Cotula piper Vellos. Compositae.

Nhandi. Tupyname für Enkea ceanothifolia Kth. Piperaceae. Ph. Rilsch., 1894, p. 241.

Nhandi-papo. Tupybenennung für Genipabeira.

Nhandiroba. Vide Jabotá.

Nhandú. Ottonia eucalyptifolia Kth. Piperaceae. Ph. Rdsch., 1894, p. 284.

Nhandúbah. Vide Aroma.

Nhanica und Nianica. Eugenia nhanica Camb. Mytaceae.

Nhatinga. Vide Becuiba branca.

Nhu mbiuva. Bauholz im Staate Parana.

Niambi. Vide Pimenta dos Indios.

Nikurioba, Nikuriroba. Vide Arikuryoba.

Nikury. Vide Aliculi. Ph. Rdsch., 1888, p. 91.

Ninga. Vide Aninga. Ph, Risch., 1892, p. 281.

Noli. Indianische Benennung für Dende da terra. Ph. Rdsch., 1889, p. 167.

Nó de porco. Schweinsknöchel. Vide Imburana.

Nopal. Vide Urumbeba.

Norma. Vide Minerva.

Novato. Bauholz in Matto Grosso.

Nove horas. Drosera intermedia Hayn. var. americana DC. Droseraceae. Ber. d. d. ph. Cies., 1897, p. 289.

Noz de Cayenna. , Alenrites molucana Willd. Enphorhiaceae. Noz da India. i Sehr häufig kultivirt.

Noz moscada das $\Lambda$ ntilhas. Muskatmuss der Antillen. Mespilodaphne moschatil Meissn. Lilunalceale. Kultivirt.

Noz moscada do Brasil. Brasilianische Muskatnuss. Cryptoraria moschatal Mart. Lanlaceae. Pham. Rev., 18!8, p. 1; '/tsch. 1. allg. oest. $\Lambda$ p.-Ver., 1868.

Noz vomica falsa. Vide Quina lo campo.

Noz vomica da term. Einhëimische Brechumss. Strarhos alhifloral Prag. Loganiaceate.

Nucapurana. Bauhol\% in Parana. 
Oaca. Vide Uaca.

O a c a jú. Vide Acajú. Ber. d. d. ph. Ges., 1898, p. 168.

Ocamaty. Vide Uva do mato.

Oajará bexiga.

Oajará preta. $\{$ Möbelhölzer im Staate Parà.

Oajará vermelha.

Oajurú. Vide Goajerú.

Oami-jurú. Salacia grandiflora Peyr. Hippocratiaceae.

Oanani, Oanini, Onany. Symphonia globulifera L. Fil. Guttifere.

Ber. d. d. ph. Ges., 1897, p. 283.

Oarari-carápia. Moutabea dibotrya Mart. Ebenaceae.

Oassacú. Vide Assacù.

() a ussú. Vide Palha branca. (Von oaú-Palme, oba-Blatt, assú gross.)

Oaxima mirim. Vide Guaxima macho. Ber. d. d. ph. Ges., 1900, p. 269.

Obajá mirim. Kleine O. Tupyname für Angelim doce.

Oca. Ockerkraut. Oxalis chrysantha Prog. Oxalidaceae. Ber. d. d. ph. Ges., 1899, p. 46.

Ocotea amargosa. Vide Sassafraz do Parà.

Ocotea aromatica. Aromatische O. Vide Canella de cheiro. Pl. Rdsch., 1898, p. 13.

Ocuba. Myristica macrophylla Spr. et Bth. Myristicaceae.

Oëra Corrumpirt von Guyra. Vide Herra de passarinho. Ph. Rev., 1900.

Oëra-repoty. Vide Guyra-tepoty (Vogelkoth). Ph. Rer., 1900.

Official da sala. Auch Ipecacuanha falsa, Mata olho. Vide Cega olho.

Oirana. Vide Salgueira do Rio (Tupyname).

Oitchi. Myrcia oitchi Bg. Myrtaceae.

Oiti, Oity. Moquilea tomentosa Bth. Rosaceae. Arch. d. Ph., 100, p. 172. Moquilea tomentosa var. latifolia Bth. Rosaceae.

Oiti bravo. Wilde O. Chrysophyllum Cearensis Fr. All. Sapotaceæ. Urostigma fulvum Miq. Lrticaceae.

Oiti cica. Soaresia nitida Fr. All. Urticaceae Ph. Rdsch., 1891, p. 219. In Pernambuco Pleragina umbracissima Arrud. Rosacer. In Rio de Janeiro Moquilea tomentosa var. angustifolia Bth. Rosaceae. 
Oiti coré.
Oiti coroya. Rauher Oiti. Moquilea rufa Barb. Rodr. Rosacex.

Oiti-guassú. Grosse O. Moquilea grandiflora Mart.; Conepia racemosa Bth. Rosaceae. Arch. d. Pharm., 82, p. 95.

Oiti mirim. Kleine $\mathrm{O}$.

Oiti da praia. Küsten O. $\}$ Pleragina odorata Arrud. Rosaceae.

Oiti do porco. Schweinsoiti. Couepia Martiana Hook. Fil. Rosaceae.

Oiti-turuba. Vide Cutitiriba.

Oixima. Vide Guaxima macho.

Okena. Keulenholz. Ouratea hexasperma var. typica Baill. Ouratea hexasperma var. Planchonii Baill. Ochnaceae.

Olandy carvalho. Vide Jacaré-huiba. Ber. d. d. ph. Ges., 1897, p. 238.

Oleo de Jatahy. Jatahyöl. Peltogyne bicolor Vog. Caesalpineacex.

Oleo de Jetahy. Jetahyöl. Peltogyne discolor Vog. Caesalpineaceæ.

Oleo de mico. Affenbalsam. Mycrocarpus frondosus Fr. All. Caesalpineaceae.

Oleo pardo, Oleo de macaco. Tolubalsam. Vide Cabureiba. Ein corrumpirtes Tupywort: Kaburé-Eule, yba-Baum, in welchem vorzugsweise Strix brasiliana Lath. nistet. Arch. d. Ph., 103, p. 309.

Oleo vermelho. Rothes Oel. Perubalsam. Myroxylon peruiferum Linn. Fil. Papilionaceae. Zeitsch. d. allg. oest. Ap. Ver., 1878 u. 1879, No. 30,$31 ; 1880$, No. 9.

Olho de boi. Ochsenauge. Mucuna urens DC. Papilionaceae.

Olho de burrico. Kleines Eselsauge. Mucuna eriocarpa Barb. Rodr. Papilionaceae.

Olho de burro. Eselsauge. Vide Olho de boi.

Olho de cabra. Ziegenauge. Vide Olho de pato.

Olho de gato. Katzenauge. Vide Arriozes.

Olho de pato. Entenauge. Ormosia coccinea Jacq. Papilionaceae. Olho de onça. Jaguarauge. Vide Olho de boi.

Olho de pomba. Taubenauge. Rynchosia phaseloides DC. Papilionaceae. In Minas Soutellaria tubiflora Bth. Labiatae. Vide Jequirity.

Oleo de Santa Luzia. Vide Marianinha.

Olmo do mato. Wilde Ulme. Homalium Racoubea Sw. Flacourtiaceae. Ber. d. d. ph. Ges.. 1899, p. 166. 
Ombú. Vide Umbú.

Onnianga pixerica, Mnianaga pixerica. Clidemia hirta D.

Don. Melastomaceae.

Onianga pixerica. Clidemia hirta D. Don. Melastomaceae.

Ora pro nobis. Gebetpflanze. Peireskia Bleo DC. Cactaceae.

Ora pro nobis de espinho. Peireskia aculeata Plum. Cactaceae.

Ora pro nobis manso. Vide Ora pro nobis.

Ora pro nobis miuda. Kleine G. Talinumn patens Willd. Portulaccaceae. Pl. Rdsch., 1896, p. 155.

Orelha de burro. Eselsohr. Clusia Cambessedesii Planch..et Trian.

Guttiferae. Ber. d. d. ph. Ges., 1897, p. 239.. Centrosema roseum Benth. Papilionaceae. Oncidium lenceanum Lind. Orchidaceae.

Orelha de gato. Katzenohr. Hypericum connatum Lam. Guttiferae. Ber. d. d. ph. Ges., 1897, p. 234.

Orelha de lebre. Hasenohr. Oxalis bupleurifolia St. Hil. Oxalidaceae. Ber. d. d. ph. Ges., 1899, p. 48.

Orelha de negro. Negerohr. Enterolobium Timbouva Mart. Mimosaceae.

Orelha de onça. Jaguarohr. Cissampelos ovalifolia DC. Menispermaceae. Arch. d. Ph., 94, p. 36t; Ph. Rdsch.. 1895. Macrolobium acaciaefolium Bth. Caesalpineaceae. Ztsch. d. allg. öst. Ap.-Ver., 1868. Echites dubia Vellos. Apocynaceae.

Orelha de onça da serra. Tibouchina holosericea Baill. Melastomaceae.

Orelha de paó. Holzohr. Polyporus sanguineus Fries. Fungi.

Orelha de raposo. Fuchsolır. Inga vulpina Mart. Mimosaceae. Orelha de rato. Rattenohr. Vandelia crustacea Bth. Scrophulariaceae. Vide auch Caa-ataya.

Orelha de urso. Bärenohr. Tibouchina villosissima Cogn. Melastomaceae.

Orelha de viado. Rehohr. Vide Taioba.

Orélia. Schmuckpflanze. Allemanda cathartica L. Apocynaceae.

Orgebão. Vide Aguará-ponda.

Orgebão de folha estreita. Schmalblättrige Gervão. Elythraria

tridentata Vahl. Acanthaceae.

Origône. Geschälte trockene Pfiirsichscheiben, in Rollen von circa $22 \mathrm{~cm}$. Durchmesser gepresst von 5 bis $10 \mathrm{k}$. Gewicht, eingehüllt in Maishülsenstroh, vom Staate Rio Grande do Sul exportirt. () robá. Bauholz in Cearà und Maranhao. 
Oröi. Stenolobium galactioides Bth. Papjilionaceate.

Orselha, Urzella. Orseille. Roccella tinctoria Ach. Lichenes.

Ortellaã brava. Wilde Minze. Hyptis loplanta Mart. Laliatide. Ortelaa de campina. Wiesenminze. Cunila menthioides Bth. Labiatiae.

Ortelaa de cheiro. Wohlriechende Minze. Myrsipanthes hovptoirles Mart. Labiatae.

f Ortela de folha lagra. Breitblätrige Minze. Vide Mlevante. $\{$ Ortelaã de mato. Waldminze. Zeitsch. d.allg.öst. Ap.-Ver., 1868.

Ortelaã de mato. Schulthesia stenophylla Mart. Gentianaciat. Ber. d. d. ph. Ges., 1899, p. 224.

Ortiga branca. Weisse Nessel. Urera punn Wedd. Crticaceile. Ph. Rdsch., 1892, p. 35.

Ortiga branca do Mato Virgem. Weisse Urwaldnessel. Urera armigera Miq. Urticaceae.

Ortiga brava. Wilde N. Urera acuminata Miq. Urticaceae.

Ortiga mansa. Zahme N.

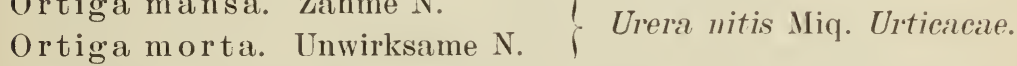

Ortiga de reino. Europäische N. Urtical nrens L. Urticaceae. Auf dem Orgelgebirge verwildert.

Ortiga vermelha. Rothe N. Urera baccifera Gaud. Urticaceale. Ortigao. Grosse N. Urera subpeltata Miq. Urticaceae.

Otoba. Myristica otoba H. B. Myristicaceae.

Pacapébuá, Pacapehía. Symplocos ciponina L’Herit. Sumplocaceae.

Pacará. Aus Palmblätter geflochtenes Körbchen der Indianer.

Pacará-tepú. Heilmittel der Indianer im Staate Amazonas.

Pacará-tucú. Tupybenennung für Jasmin do mato.

Pacari. Lafoensia Pacari St. Hil. Lythraceae. In Minas Lafoensia replicata Pohl. var. Lundii Koehn. Lythraceae.

Pacari do campo. Steppen P. Lafoensia densiflora Pohl. Lythraceae.

Pacari do mato. Polygala Gardneriana A. W. Benn. Polygalaceae. Pacariva. Securidaca Sellowiana Klzch. Polygalaceae. Pacharó-lobo. Tessaria absinthioides DC. Compositae. Pachaú, Р’ахаи, Pajahú. Triplaris pachau Mart. Polygonaceae. Ph. Rdsch., 1895, p. 36. 
Pachurú. Vide Icaco, Goajerú.

$\left.\begin{array}{l}\text { Pacoba. Tupyname für Banana da terra. } \\ \text { Pacob-assú. Tupyname für Banana. }\end{array}\right\}$ Ph. Rdsch., 1894, p. 37.

Tacoa-yre. Tupyname für Bananenstamm.

Pacoburá. Tupyname für den Baum.

Paco-caatinga, Pacova. Renealmia exaltata L. fil. Zingiberaceae.

Ph. Rdsch., 1893, p. 288. Vide auch Ganna roxo do brejo.

Pacory, Pacuriúriuva. Indianische Benennung für Bacapari.

\{ Pacova. Tupyname fuir Cardamomo sylvestre.

\{ Pacova-seróca.

Pacovà. Tupyname für Pimentão comprido.

Pacúan. Indianisches Heilmittel in Amazonas.

Pacúa-rana. Canna lanuginosa Rosi. Cannaceae. Ph. Rdsch., 1893, p. 257.

Pacúri. Pacourea guianensis Aubl. Apocynacea.

Pahni. Artanthe Velloziana Miq. Piperaceae. Ph. Rdsch., 1894, p. 285.

Pajanerioba. Heilmittel in Pará.

Paida. Brosimum Anblettii Popp. Urbicaceae.

Paina abobora. Kürbissbaumwolle (zufolge der Fruchtgrösse).

Fischeria adenophylla Fourn. Asclepiadaceae.

Paina loura. Blonde Samenwolle. Vide Cipó de pennas.

Paina melão. Melonenbaumwolle (zufolge der Fruchtform). Fischeria Martiana Fourn. Asclepiadaceae.

Paina de pennas. Federbaumwolle. Echites peltata Vell. Apocynaceae.

Paina seda branca. Weisse Seidenbaumwolle. Gomphocarpus brasiliensis Fourn. Asclepiadaceae.

Paina seda do campo. Seidenwolle der Steppe. Oxypetalum campestre Don. Asclepiadaceae.

Paina velluda. Sammtbaumwolle. | Peckoltia pedalis Tourn. Painasinha. Kleine " f Asclepiadaceae.

Paineira. Wollbaum. Bombax stenopetalum Schum. Bombaceae. Ber. d. d. ph. Ges., 1900, pp. 155-165.

Paineira anao. Zwergwollbaum. Bombax marginatum var. nana Schum. Bombaceae. Ber. d. d. ph. Ges., 1900, pp. 155-165.

Paineira amarella das pedras. Gelber Wollbaum auf Felsen. Bombax campestre Schum. Bombaceae. Ber. d. d. ph. Ges., 1900, pp. $155-165$. 
Paineira arbusto. Wollstrauch. Bombax marginatum Schum. Bombaceae. Ber. d. d. ph. Ges., 1900, pp. 155-165.

Paineira barriguda. Bauschiger Wollbaum. Chorisia insignis HBKth. Bombaceae. Ber. d. d. ph. Ges., 1900, pp. 15.5-16.5.

Paineira branca. Weisser Wollbaum. Ceiba erianthos Schum. Bombaceace. Ber. d. d. ph. ('es.. 1900, pp. 15.5-165.

Paineira branca das pedras. Weisser Felswollbaum. Bombix pentaphyllum Vell. Bombaceae. Ber. d. d. ph. Ges., 1900, pp. $155-165$.

Paineira do cainpo. Steppenwollhaum. Bombax cremulatum Schum. Bombaceate. Ber. d. d. ph. Ges., 1900, pp. 15.)-1(i;).

Paineira carneiro. Vide ('arneira.

Paineira cheirosa. Wohlriechender Wollb. Ceiba jasminodora Schum. Bombaceae. Ber. d. (l. ph. Ges., 1900, p. 161.

Paineira cipo. Lianenwollpflanze. Physianthes albens Mart. Asclepiadaceae.

Paineira de Cuba. Vide Castanha de Cearà. P. 167.

Paineira femea. Weiblicher Wollbaum. Chorisia speciosa St. Hil. Bombaceae. Ber. d. d. ph. Ges., 1900, pp. 155-164.

Paineira de harpoador. Harpunirwollb. Bombax cyathophorum

Schum. Bombaceae. Ber. d. d. ph. ph. Ges., 1900, pp. 15.)-165.

Paineira imbir-assú. Vide Imbir-assú.

Paineira macho. Männlicher Wollbaum. Chorisia Peckoltiana

Mart. Bombaceae. Ber. d. d. ph. Ges., 1900, pp. 155-164.

Paineira da praia. Küstenwolle. Vide Paineira à tôa.

Paineira seda. Seidenwollb. Oxypetalum campestre Don. Asclepiadaceae.

Paineira à tôa. Nutzloser Wollb. Bombax endecaphylhum Vell. Bombaceae.

Painerinha. Kleiner Wollb. Vide Paineira anão.

Painzo, Milho painzo, Milho miudo. Hirse. Panicum miliaceum L. Gramineae. In den Südstaaten kultivirt.

Pajo marióba. Cassia patellaria DC. Caesalpineaceae.

Pajurá. Licania triandra Mart. Rosaceae.

Pakarà. Echinopsis oxygona Zuce. C'actaceae.

Palata. Lncuma mammosa Gärtn. Sapotaceae. Ph. Rdsch., 1888, p. 6. 
Palha branca. Weisses Stroh. Attalea speciosa Mart. Palmae. Ph. Rdsch., 1889, p. 133. Diplothemium pectinatum Barb. Rodr. Palmae.

Palilho. Stockbaum. Campomanesia lineatifolia Ruiz et Pav. Myrtaceae.

P alma. Vide Catinga de mulata.

Palma do campa. Steppenpahme. Cassia uniflora Spreng. Caesalpineaceae.

Palma real. Vide Buriti.

Palma do rosario. Rosenkranzpalme. Vide Assai mirim. Pl. Rdsch., 1889, p. 193.

Palmatoria. Handschläger. Vide Urumbeba.

Palmeira. Palmbaum. Attalea compta Mart. Palmae. Ph. Rdsch., 1889.

Palmeira de defunto. Todtenpalme. Cycas revolutum L. Cycadaceae. Ph. Rdsch., 1893, p. 133.

Palmeira real. Königspalme. Oreodoxa oleracea Mart. Palmae. Ph. Rdsch., 1889, p. 194.

Palmeira real da terra. Einheimische Königspalme. Cocos pityrophylla Mart. Palmae. Ph. Rdsch., 1889, p. 92.

Palmeirim. Kleine Palme. Vide Coqueiro Catolé.

Palmito. Kohlpalme. Euterpe edulis Mart. Palmae. Ph. Rdsch., 1889, p. 191. Vide auch Assai.

Palmito amargoso. Bittere Kohlpalme. Vide Paty amargoso. Palmito molle. Zarte Kohlpalme. Vide Assai mirim.

Palové. Eperua fialcata Aubl. Caesalpineaceae.

P a mon ân. Indianer'speisevorrath für lange Reise. Masse von Fleisch,

Fisch, Mandioceamehl, etc.

Pamónha. Gebäck ron Mandioccamehl, etc.

Panacea. Vide Braço de preguiza. Arch. d. Pharm., 97, p. 103.

Panamí. Vide Herva de Santa Maria.

Pananáma. Auch Barboleta do mato. Oxalis elatior Prog. Oxalidaceae. Ber. d. d. ph. Ges., 1899, p. 47.

Paná-paná-ré und Panna-pá-nari. Quapoya pannapanari Aubl. Guttiferae.

Panduraní. Vide Pacuarana.

Paneira. Vide Tipiti.

Pang6. Negerbenennung für Cannabis asiatica L. Irticaceae.

Pani. Artanthe geniculata Miq. Piperaceae. Ph. Rdsch., 1894, p. 286. 
Pao de ló de mico. Affenkuchen. Das Mark der Friichte von Jetahy. Páo. Baum. Ausgesprochen Paú.

Páo d’alho. Knoblauchbaum. Vide Tapia und Gorarema.

Páo d'alho do campo. Steppen K. Agonandra brasiliensis Miers.

Olacinaceae. Ber. 1. d. plı. Ges., 1901, p. 47.

Pá o a marello. Gelbholz. Vochysia obscura Warm. Vochysiaceae.

Ferner haben noch folgende diese Benennung: Arapoca amarello,

Guarubí amarello, Tatagiba und Vinhatico testa de boi.

Pá o a margoso. Bitterholz. Vide Angelim amargoso und Quassia. Páo d'arco. Bogenholz. Tecoma pentaphylla Juss. Bignoniaceare.

In Amazonas Curalia toxophora Bth. et Hook. Bignoniaceae. Ist das Guira-pariba des Marcgraf.

In Pernambuco Tecoma leucoxylon Mart. Bignoniareae.

In S. Paulo Tecoma aurea P. DC. Bignoniaceate.

Páo d’arco am arello. Gelbes B. Tecoma rhrysostricha Mart.

Bignoniareae.

Páo d'arco do campo. Steppen B. Tecomal Caraiba Mart. Big-

noniaceae. Heisst auch Caraiba.

In Minas Tecoma ochracea Cham. Bignoniaceae.

Páo d'arco roxo. Vide Ipé roxo.

Páo d'azeite. Oelbaum. Vide Lantim.

In Minas Ilex acrodonta Reiss. Ilicinaceae.

Pá o de azude. Mïhlendammholz. Bauholz in Maranhão.

Pá o balla. Kugelholz (wegen des Büchsenkugelähnlichen Samens).

Guarea trichilioides L. var. pachycarpa C. DC. Meliaceae. Ber. d.

d. ph. Ges., 1901, p. 32t.

Pá o bałsamo. Balsambaum. Vide Anguay und Oleo de mico.

Pá o barriguda. Dickbäuchiger B. Chorisia crispiflora HBKth.

Bombaceae.

Pá o boaz. Flötenholz. Piptadenia falcata Bth. Minısaceae.

Pá o branco. Weissholz. Cordia oncocalyx Fr. Allem. Boraginacex.

Ber. d. d. ph. Ges., 1902. Phyllostylon brasiliensis Capan. Urticaceae. Ph. Rdsch., 1892, p. 34.

Páo Brasil. Brasilholz. Caesalpinia echinata Lam. Caesalpineacex.

Spec. Gew. 1.129. Arch. d. Pharm., 91, p. 110.

Páo Brasil falso. Falsches Brasilholz. Caesalpinia peltophoroides

Bth. Caesalpineaceae.

Páo bravo. Schädlicher Baum. Aspidosperma Spruceanum Bth. Apocynaceae. 
Páo bréo. Colophoniumbaum. Protium divaricatum Engl. Burseraceae. Arch. d. Pharm., 93, p. 119; Ber. d. d. ph. Ges., 1898, p. 443. Vide auch Peineira do campo.

Páo de cachimbo. Pfeifenbaum. Vide Sapucainha.

Páo de cachorro. Hundsbaum. Vide Ipé contra sarnas.

Páo de caninana. Hundsschlangenbaum. Vide Cainea.

Páo de caixeta. Kistchenholz. Tide Bandarrá.

Páo de camarao. Krabbenbaum. Clydostoma noterophilum Bur.

et K. Schumann. Bignoniaceae.

Páo de candeia. Leuchterbaum. Piptocarpha Vautheriana Bak. Compositae.

Pá o de canella. Zimmtbaum. Vide Canella caixeta. Pharm. Arch., 1898, p. 12.

Páo de cantil. Fässchenbaum. Cupania vernalis Camb. Sapindaceae Ber. d. d. ph. Ges., 1902.

Páo Cardazo. Distelartiger Baum. Alsophila atrovirens Presl.

Cyatheaceae. Arch. d. Pharm., 102, p. 168.

Pá o de carga. Prügelholz. | Casearia grandiflora St. Hil. FlaPá o de carne. Fleischbaum. f courtiaceae. Ber. d. d. ph. Ges., 1899, p. 173.

Pá o cavallo. Pferdebaum. Vitex multinervis Schauer. Verhenaceæ. Páo cobra. Schlangenbaum. Simaba snaveolens St. Hil. Simarubaceae. Ber. d. d. ph. Ges., 1898, p. 428.

Páo de cobra cipo. Schlangenliane. Vide Pimenta de cobra cipo. Páo de colher. Löffelholz. Tabernamontana Salzmanni A. DC. Apocynaceae.

In Minas Maytenus rigida Mart. Celastrinaceae.

Páo de coral. Korallenbaum. Erythoxylon corallodendron Fr. Allem. Erythoxylaceae.

Páo cravo. Gewürznelkenbaum. Vide Craveira de Maranhao.

Páo de cruz. Kreuzbaum. Prockià crucis L. Flacourtiaceae. Ber. d. d. ph. Ges., 1899, p. 166.

Páo do diabo. Teufelsbaum, heisst auch Cura madre. Gebärmutterheilmittel. Guarea tuberculata Vell. var. purgans D. C.; 1 richilia hirsuta C. DC. Meliaceae. Ber. d. d. ph. Ges., 1901, pp. 351, 353. Páo doce. Süsser Baum. Vochysia rufa Mart.; Vochysia tucanorum Mart. Vochysiaceae. Ferner Guaranhem and Folha larga.

Páo de embira. Bastbaum. Vide Lappa vermelha und Pindahyba. Páo de espeto. Bratspiessholz. Vide Mathias. 
Páo de espinho. Dornbaum. Maclura xanthoxylon Endl. Liticareap.

Heisst auch Amoreira de espinho-Dorniger Maulbeerbaum, und

Espinheiro branco - Weisser Iornenbaum. Ph. Rdsch., 1891,

p. 291.

Páo facho. Fackelholz in Alagoas. Bignoniaceate.

Páo faia. Buche. Bauholz in Alagoas.

Páo fedorento. Stinkbaum. Vide Canella fedorenta. Ph. Arch., 1898 , p. 27.

Páo ferro. Eisenholz. Caesalpinial ferrea Mart. C'aesalpiniaceale.

- In Amazonas Callophyllum pachyphyllum Planch. et Trian. Giuttiferae. Ber. d. d. ph. Ges., 1897, p. $2: 38$.

In Cearà Dialeum divaricatum Vahl. C'aesalpineaceae.

In Minas Machaerium sideroxylon Tul. Papilionaceae.

Páo ferro do cain po. Steppeneisenholz. Vide Arâryba do campo.

Ph. Arch., 1901, p. 53.

Páo de foquete. Racketenbaum. Marcgravia rectiflora Fr. et

Planch. Marcgraviaceae.

In Cearà Marcgravia picta Willd. Ber. d. d. ph. Ges., 1901, p. 94.

Páo de formigá. Ameisenbaum. Cordia nodosa Lan. Boragi-

nareae. Ber. d. d. ph. Ges., 1902.

Páo de forquilha. Gabelbaum. Vide Páo Pereira.

Páo de gentio. Indianerbaum. Crticaceae?

Páo gerimú. Kürbissbaum. Pisonia pubescens HBKth. Nyctaginaceae. Pharm. Rev., 1896, p. 81.

Páo de gomma. Gummibaum. Vide Araxina.

Páo de guiabo. Quingombobaum. Oreodaphne Coto Nees. Lan-

raceae. Pharm. Rev., 1898, p. 19.

Pá o homens. Männerbaum. Vide Marapuama.

Páo Guiné. Guineabaum. Vide Fructo do Conde do mato. Ber.

d. d. ph. Ges., 1897 , p. 454.

$P$ á de I angada. Vide Iangadeira.

Páo de imbira. Bastbaum. Xylopia ligustrifolia Dan. Anonaceae.

Ber. d. d. ph. Ges., 1897, p. 465.

Páo de incenço. Weihrauchbaum. Vide Incenço (Protiumı).

Páo de judeo. Judenbaum. Vide Joao molle.

Páo lacre. Firnissbaum. Vismia latifolia Chois. Guttiferae. Ber.

d. d. ph. Ges., 1897, pp. 235, 236. Vide auch Caa-opia.

Páo de lagarto. Eidechsenbaum. Apodanthes caseareae Pois.

Rafflesiaceae. 
Páo de lagarto legitimo. Aechter Eidechsenbaum. Casearia adstringens Mart. Flacourtiaceae. Ber. d. d. ph. Ges., 1899, p. 172. Páo de lagôa. Seebaum. Vide Tabùa.

Páo de leite. Milchbaum. Vide Tiú.

Páo de lepra. Aussatzbaum. Vide João molle.

Páo de letras. Buchstabenbaum. Helicostrlis Poeppigiana Trec. Lrticaceae. Ph. Rdsch., 1891, p. 219.

Páo lixa. Polirbaum. Vide Lixa.

Páo manteiga. Butterbaum. Bauholz in Pernambuco.

Pá o maqueira und II oquem. Fleischräucherholz. Eupatorium Burchelli Bak. Compositae.

Pá o Il a ria. Marienbaum. Vide Lantim.

Pán de mocó. Korbbaum. Tipuana auriculata Fr. Allem. Papilionaceae.

Páo de móco und míco. Schleimbaum. Lonchocarpus speciflorum Mart. Papilionaceae.

Páo molle. Weiches Holz. Vide João molle und Abaremo-temo. In Rio de Janeiro Vitex polygama Cham. Terbenaceae.

Páo de monjólo. Vide Monjólo.

Páo mondé. Pisonia suhcordata Sw. Nyctaginaceae. Pharm. Rev., 1896, p. 81.

Pá o de morcega. Fledermausbaum. Vide Angelim de follha larga. Páo de mulato. Mulattenbaum. Vochvsia micrantha Pohl. Vorhysiaceae.

Páo de mulato und auch Capirona. Calicophyllum Spruceanum Hook. fil. Rubiaceae.

Páo negro. Negerbaum. Heisst auch Coraçao de negro-Negerlierzbaum. ('arroeira - Kohlenbaum. Albizzia Labbeck T. Mimosaceae. Eingefiihrt, jetzt verwildert.

Páo de novato. Triplaris surinamensis Cham. var. Benthamiana Meissn. Polvgoniaceae. Ph. Rdsch., 1895, p. 36.

Páo de oleo. Oelbaum. Vide Cabureiba und Oleo pardo.

In Pernambuco Copaifera officinalis L. Caesalpineaceae.

Páo papel. Papierbaum. Tibouchina papyrifera Cogn. Melastomaceae.

Páo Parahyba. Farbiges Holz. Vide Paraiba.

Páo pedra. Steinholz. Tide Páo de serrote (Moldenhauera). 
Pá o pente. Kammbaum. | Geissospermum Vellozii Fr. Allem. ApoPáo perara. Birnbaum. f cynaceae. Arch. d. Ph., 97, p. 102 ; '/tschr. d. allg. öst. Apoth.-Ver., 1896, pp. 34, 35.

Páo pareira mansa. | Picramnia ciliata Mart. Simarubaceae. Páo perura da serra. J Ber. d. d. ph. Ges., 1898, p. 436.

Páo pilao. Stampfmiihlenholz. Callisthene minor Mart. Vochysiacese. Pá o pimenta. Pfefferbaum. Vide Craveira de Maranhao.

Páo pintado. Gefleckter Baum. Vide Angelim côco.

Páo piranga. Rothholz. Laetia apetala Jacq. Hlacourtiaceae.

Ber. d. d. ph. Ges., 1899, p. 167.

Páo pobre. Schlechtes Holz. Enphorbiaceae in Minas.

Páo podre. Faules Holz. Didodendron lipinnatnu Radk. Sápindaceae. Ber. d. d. ph. Ges., 1902.

Páo pombo. Taubenbaum. Vide Marupay. Auch Cachua branca. Matayba juglandifolia Radk. Sapindaceae; Borreria eupatorioides Cham. et Schlecht. Rubiaceae; Phoradendron P'erottetii DC. Loranthaceae. Vide Liga-Liga.

In Minas Tapirira Marchandii Engl. Anacardiaceae.

In den Nordstaaten Tapirira guianensis Aubl. Inacardiaceae.

In S. Paulo Tapirira guianensis Aubl. var. cuneata Engl. Anacardiaceae. Ber. d. d. ph. Ges., 1898, p. 157.

Páo de porco. Schweinsbaum. Trattinickia burseraefolia Mart.

Burseraceae. Ber. d. d. ph. Ges., 1898, p. 444.

In Minas Rourea induta Planch. Connaraceae. Ph. Rev., 1901, p. 46. Vide auch Páo pombo (Borreria).

Páo praga. Unkraut. Vide Cordao de frade.

Páo precioso. Kostbarer Baum. Vide Casca preciosa. Pharm. Arch., 1898.

Páo preto. Schwarzes Holz. Killmeyera excelsa St. Hil. Guttiferæ. Ber. d. d. ph. Ges., 1897, p. 229. Inga laterifolio Miq. Mimosaceæ.

-Vide auch Cabiúna und Jacaranda preta (Dalbergia).

Páo puante. Stinkbaum. Gustavia augusta L. Myrtaceae.

Páo queimadeira. Brennbaum. Stenolobium arboreum Wawra.

Papilionaceae.

Páo rainha. Königinbaum. Cetrolobinm paraense Tul.Papilionacex. Páo de rato. Rattenbaum. Guariba turbinata Poir. Bombaceae. Ber. d. d. ph. Ges., 1900, p. 170.

Páo de rato dos sertanejos. Caesalpenia microphylla Mart. Caesalpiniaceae. 
Páo rei. Königsbaum. Basiloxylon brasiliensis K. Schum. Sterculiaceae. Ber. d. d. ph. Ges., 1900, p. ј6.

Páo de remos, auch P. branca de remos. Weisses Rudernholz. Urwaldbaum in Maranhao.

Páo rosa. Rosenholz. Aydendron riparium Nees. Lauraceae. Ph. Arch., 1898. Peltogyne discolor Vog. Caesalpiniaceae. Hernandia guianensis Aubl. Hernandiaceae.

Vide ferner Grao de poreo (Physocalymna), sp. Gew. 0,894. Sebastiao de Arruda und Oraveira de Maranhao.

Páo rosado. Rosafarbenes Holz. Vide Páo Brasil.

Páo de rosario. Rosenkranzbaum. Vide Catagía.

Páo de roseira. Rosenbaum. Vide Roseira do mato.

Páo roxo. Violettrother Baum. Vide Guarnbú royo.

Páo de sabao. Seifenbaum. Sapindus saponaria L. Sapindaceae.

Ber. d. d. ph. Ges., 1902. Assafrôa hat auch diese Benennung.

Páo de sangue. Blutbaum. Vide Becuiba. Arch. d. Ph., 108, p. 14.

Sangue de drago und Urúcuba.

Páo de Santa Anna. Heiliger Annenbaum. Vide Canella preta da serra. Pharm. Arch., 1898, p. 24.

Páo santo. Heiliger Baum, auch Mahuba. Mahnrea palustris

Aubl. Guttiferae. Ber. d. d. ph. Ges., 1897, p. 228. Vide auch

Malva de campo und Ipé tabaco.

Páo santo de 7 nomens. Heiliger sieben Männerbaum. Vide

Páo preto (Kielmeyera).

Páo de Sao .Jose. Heiliger Josephbaum. Kielmeyera coriacea

Mart. Guttiferae. Ber. d. d. ph. Ges., 1897.

Páo sassafras. Mespilodaphne indecora var. canella Meissn.

Lauraceae. Ph. Arch., 1898, p. 15. Vide auch Sassafraz do Parà.

Páo de semana. Wochenbaum. Vide Mareci (Byrsonima).

Páo de seringa. Klystirspritzenbaum. Vide Borracha.

Pá o de serpente. Schlangenbaum. Vide Páo de lebras.

Páo de serra. Sägebaum. Vide Jabotapita mirim.

Páo de serrote. Handsägebaum. Vide Páo de móco. Moldenhauera cuprea Pohl. Caesalpiniaceae.

Páo de setim. Seidenholz. Aspidosperma eburneum Fr. Allem. Apocynaceae. Sp. Gew. 0,845. Esenbeckia fasciculata Barb. Rodr. Rutaceae.

Páo de sombra. Schattenbaum. Vide Anga-iba-atan. Ber. d. d. ph. Ges., 1902. 
Páo de tamancos. Pantoffelholz. Vide Fabebuia do brejo.

Páo de tanho branco. Weisses Sesselholz. Vide Páo de setim und Pequia marfim.

Páo de tartaruga. Schildkrötenbaum. Brosimum Aubletii Popp. Urticaceae.

Páo terra. Feldbaum. Qualea grandiflora Mart. Vochysiaceae.

Páo terra do campo. Steppenfeldbaum. Vide Uvapuva do campo.

Páo terra grande. Grosser Feldbaum. Callisthene maior Mart.

Vochysiaceae.

Páo terra do mato. Wilder Feldbaum. Callisthene maior Mart. Vochysiaceae.

Páo terra pequena. Kleiner F. Qualia parviflora Mart. Tochysiaceae.

Páo tingui. Fischgiftbaum. Magonia pubescens St. Hil. Sapindaceae. Ber. d. d. ph. Ges., 1902.

Páo de trombeta. Trompetenbaum. Vide Ambaiba. Pl. Rdsch., 1891, p. 289.

Páo de tucano. Vochysia Ancanorum Mart. Vorhysiaceae.

Páo de vacea. Kuhbaum. Vide Massaranduba.

Páo de varetas. Ruthenbaum. Aegiphila arboresiens Vahl. Verbenaceae.

Páo velho. Greisenbaum. Capia aponconiea Aubl. Caesalpiniaceae. Páo de viola. Guitarrenbaum. Vide Fabebuia do brejo.

Páo de violeta. Veilchenbaum. Machaerium violaceum Vog. Papilionaceae.

Páo de Yuca. Vide Páo ferro (Machaerium).

Páo de zebra. Zebraholz. Connarus guianensis Lamb. Connaraceae. Ph. Arch., 1901, p. 51.

Páolin. Coroadenindianer Namen für Attalea compta Mart. Palmae. Papaconha. Hybanthus ipecacuanha Taub. Violaceae. Ber. d. d. ph. Ges., 1897, p. 100.

Papagaio. Vide Ponceta und Tinhorao.

Papa de anjos. Engelspeise. Vide Sapucainha.

Papa guela. Schlundschliesser. Gomidesia Cascarettiana Bg. Myrtaceae.

Papam undo. Allerweltsbrei. Melicocca lepidopetala Radlk. Sapindaceae.

Papa terra. Bodenverderber. Basanacantha spinosa var. typica Schum. Rubiaceae. 
In Minas. Rhynchantera dichotoma DC. Melastomaceae.

In Parà. Douradinha (Waltheria).

In Pernambuco. Caa-ataga.

Paparajúba in Maranhao. Vide Caroa.

Paparaúba. Vide Parahyba.

Papaya. Tupyname für Mamoeira.

Papéa quassú. Luphea speciosa Willd. Tiliaceae. Ber. d. d. ph. Ges., 1898, p. 284.

Papeira. Kropfpflanze. Tournefortia hirsutissima L. Borraginaceae. Ber. d. d. ph. Ges., 1902.

Papo de gallo. Hahnenkropf. I Aristolochia cymbifera Mart. var. Papo de perú. Truthalınkropf. $\{$ genuina Mart. Aristolochiaceae. Ph. Rdsch., 1893, p. 183. Vide auch Herva de tostao.

Papo de mutum. Bauholz in den Nordstaaten.

Papo de pavao. Pfauenkropf. Combretum Loeffingii Euhl. Combretaceae.

Papôco in Ceara für Pipoca.

Papoila. Mohn. Papaver rhoeas L. Papaveraceae. Angebaut.

Papoula. Mohnblume. Hibiscus mutabilis L. Malvaceae. Ber. d. d. ph. Ges., 1900, p. 271.

Papúco. Benennung in Bahia für Batuéra.

Papúdo. Dickkröpfiger. Vide Páo de diabo (Guarea).

Paquam. Gramineae. Futtergrass.

Paquerette. Bellis perennis L. Compositae. Gartenpflanze.

Paracary. Verbena paricari Fr. Allem. Verbenaceae. Arch. d. Ph., 100, p. 42.

Paracary do brejo. Sumpi P. Herpestris gratioloides Bth. Scrophularinae.

Paracary do sertao Wiisten P. Tetratilacium veronicaeforme Turz. Scrophularinae.

Paraca-úba. Tupyname für Angelim doce.

Parahiba, Paraiba. Tupy von pará-farbig, iba-Holz. Simaruba versicolor st. Hil. Simarubaceae. Ber. d. d. ph. Ges., 1894, p. 431.

Parafuso do campo. Steppenschraube. Vide Saccarolha.

Paralá. Diospyros paralea Steud. Ebenaceae.

Paramacá. Astrocaryum Paramaca Mart. Palmae.

Paranan. Vide Imbari.

$\left.\begin{array}{l}\text { Paranuari. } \\ \text { Parapana. }\end{array}\right\}$ Bauhölzer im Staate Amazonas. 
Pará-pará. Vide Páo de sombra.

Pará-pará-iba. Triplaris macrocalyx Casar. Polygonaceae. Ph.

Rdsch., 1895 , p. 36.

Paraparauba. Vide Carôa.

Paraperiúba. Mimosa lacerans Bth. Mimosaceae.

Parari. Heilpflanze der Indianer in Amazonas.

Parati. Volksbenennung für Zuckerbranntwein.

Paratucú. Vide Jasmin do mato.

Paratudo. Gut für Alles. Gomphrena officinalis Mart. Amaranthalceae. Ph. Rdsch., 1895, p. 90.

In Alagoas vide Baína. Ber. d. d. ph. Ges., 1901.

In S. Paulo vide Casca d'anta. Ber. d. d. ph. Ger., 1896, p. 192.

In Piauhy vide Corongó.

In Rio de Janeiro vide Casca para tudo. Ber. d. d. ph. Ges., 1899, p. 352.

Paratudo bravo. Wilde. Ophrys arachnoides Vell. Orchidaceite. Paratudo do campo. Steppen. Datiscal crassifolia Datiscaceae. Paratudo da praia. Kiisten. Gomphrena polygonoides Moq. Amaranthaceite.

Paratudo do sertão. Wïsten. Gomphrena leucocepliala March. Amaranthaceae.

Paratíra. Remirea maritima Aubl. Cyperaceate.

Paraúachy, Paraúakochy, Paracuaxi. Vide Paricá (Pentaoletra).

Paraúva. Securidaca Sellowiana Klotzch. Polygalaceae.

Paricá. Pentacletra filamentosa Bth. Mimosaceae.

In Rio de Janeiro Piptadenia peregrina Bth. Mimosaceae.

Paricá-cache. Rauhe P. Pentacletra brevipila Bth. Mimosaceae. Paricá legitima. Aechte P. Mimosa acacioides Miq. Mimosaceae. Paricá preta. Schwarze P. Acacia riparia HBKth. Mimosaceae. Paricá-rana. Falsche P. Stryphnodendron floribundum Bth. Mimosaceae.

Paricá-rana branca. Weisse falsche P. Acacia polyphylla DC. Mimosaceae.

Parietaria vermelha. Rothe P. Gesnonimia boehmerioides Miq. Urticaceae. Ph. Rdsch., 1892, p. 36.

Parina. Vide Paco-caa-tinga.

Parinari, Pary-nary. Couepia chrysocalyx Bth.; Parinarium brasiliense Hook. fil. Rubiaceae. 
Pariparoba. Grosses Blatt. Potomorphe umbellata Miq. Piperaceae. Vide auch Caa-peba. Artanthe Mikaniana Miq. Piperaceae. Ph. Rdsch., 1894, pp. 241, 242.

Pariquity, Priquity. Canna glauca L. Cannaceae. Ph. Rdsch., 1893 , p. 257.

Parov-assú. Grosser P.

Parov-mirim. Kleiner junger P. $\}$ Vide Perobinha do campo.

Parova-tuum-tumune. Baum, welcher regnet. Tupyname für Barbatimão.

Parova-una, Parovuna. Tupyname für Barahuna.

Parreira brava. Wilde Weinrebe. Vide Abutua.

Parreira do mato. Waldrebe. Securidaca bialata Bth. Polygalaceae.

Parurú. Bunchosia fluminensis Gr. Malpighiaceae.

Passari. Cuphea calophylla Cham. et Schlecht. Lythraceae.

Passocá. Speisevorrath der Indianer für Reise etc., eine Mischung von geröstetem Fleisch mit Mais- oder Mandioceamehl. Im Staate Parà ein Confekt von gerösteten Nïissen der Bertholletia excelsa mit Zucker und Mandioceamehl.

Pasta. Vide Gigogo.

Patagúa. Limnanthemum microphyllum Griseb. Gentianaceae. Ber. d. d. ph. Ges., 1899, p. 232.

Patahé, Patoua, Patavía. In Alagoas ein Fruchtbaum des Urwaldes, dient den Indianern zur Nahrung.

Pataqueira. Linaria canadensis Spr. Scrophulariaceae.

Pataúa, Patovouá, Patava. Vide Batauá. Ph. Rdsch., 1889, pp. 37, 38, 90, 110, 193.

Pati, Paty. | Cocos oleracea Mart. Palmae.

Pati a margoso. $\int$ Cocos Mikaniana Mart. Palmae. Ph. Rdsch., 1889, pp. 37, 38, 90, 110, 193.

Pati doce. Süsser P. Cocos botryophora Mart. Palmae.

Patioba. $\quad$ Nicht in Bahia. Pl. Rdsch., 1889, p. 37, 38, 90,

110, 193. In Bahia vide Buri.

Patioba mirim. Kleine P. Geonoma platycaula Dr. et Trl. Palmæ. Ph. Rdsch., 1889, pp. 37, 38, 30, 110, 193.

Patioba bedeutet in der Tupysprache ein ganzes zum Dachdecken brauchbares Palmblatt.

Patima-rana. Patima guianensis Aubl. Rubiaceae.

Pati-puta. Vide Bati-puta. 
Patúarana. Vide Pacúarana.

Patúatiba. Vide Jútía. Vide Ber. d. d. ph. Ges., 1901, p. 324.

Pá-ína. Dunkles Blatt in Tupy. Cardiospermum halicacalum L.

Sapindaceae. Ber. d. d. ph. (ies., 1901, p. 150.

Paupeira. Hundskohl. Vide Chuvirangana.

Paxiúba. Vicle Baxiúba.

) Ph. Rolsch., 1889,

Paxiúba majerona. Vide Baxióba majerona. $\int$ pp. 263 u. 35.

P а уайагí. Indianergetränk von Beijúkuchen in jeden beliebigen

Fruchtsaft zertheilt.

Payérioba. Tupyname für Fedegosa.

Pé de bezerro. Kalbsfuss. Vide Timhoriõo.

Pé de burro. Eselshuf. Knolle Heilmittel in Mlagoas.

Pé de galinha. Hühnerfuss. Cynodon dactylon Pers.; Dactyloctanum mucronatum Willd. Gramineae.

Pé de macaco. Affenfuss. Selaginella flexuosal Siprg. Lycopodiacean.

Pé de mico. Aeffehenfuss. Sicyos polyacanthos Cogn. Cucurbitacese.

Pé de pato. Entenfuss. Phyllinthus lathyroides var. microphyllus Müll. Arg. Euphorloiaceae.

Pé de perdix. Rebhuhnfuss. Vide Alcanforeira und Curalleira.

In Maranhao vide Parahiba.

Pécacuem. Tupyname für Cissampelos glaberrima St. Hil. Menispermaceae.

Pecegueira. Pfirsichbaum. Prunus persica Sieb. et Zuce. Rosaceæ.

Kultivirt.

Pecegueira do mato. Vide Gingeira brava.

Pechi-y. Captalia piloselloides Back. Compositae.

Pecirica. Vide Mexerico.

Pecoso. Croton catinganus Müll. Arg. Euphorbiaceae.

Pega-pega. Doppelfalle. Mentzelia fragilis Hub. Losaceae.

Pega-pinto. Küchleinfalle. Vide Herva tostão.

$\left.\begin{array}{l}\text { Peipecava. } \\ \text { Peipezava. }\end{array}\right\}$ Tupyname für Scoparia dulcis L. Scrophularineae.

Peireiró. Vide Casca preciosa.

Pellado. Vide Araxina.

Pematim. Sclerolobium paniculatum Vog. var. rubiginosum Bth. Caesalpiniaceae.

Penaibá, Penoabú. Sapium aucuparium Jacq. Euphorliaceae. Pendao de brejo. Sumpfähre. Haynaldia thapsoides Kun. Lobeliaceae. 
Pennacho amarello. Gelber Federbusch. Stiftia chrysantha Mik. Compositae.

Penon. Caoutchoucbaum in Pernambuco. Euphorbiaceae.

Pente de macaco. Affenkamm. Pithecotemum echinatum K. Schum. Bignoniaceae.

In Amazonas Apeiba Petoumo Aubl. Tiliaceae. Ber. d. d. ph. Ges., 1898, p. 283.

Pentieira. Kammbaum. Vide Caimbé bravo.

Pepino. Gurke. Cucumis sativus L. Cucurbitaceae.

Pepino de burro. Eselsgurke. Vide Machisca.

Pepino grande do mato. Grosse Waldgurke. Melothria cucumis

Vell. Cucurbitaceae.

Pepino do mato. Wilde Gurke. Solanum sisymbriifolium Lam.

Solanaceae. Sicyos Martii Cogn. Cucurbitaceae.

In Parà Ambelania tenuiflora Müll. Arg. Apocynaceae.

Pepino de papagaio. Papageiengurke. Gurania multiflora Cogn.

Cucurbitaceae.

Pepino de S..Gregorio. Heilige Georgsgurke. Vide Buxa.

Pepino da terra. Einheimische Gurke. Vide Pepino do mato (Sicyos).

Pequea-rana. Falsche P. Bauholz. Sapindaceae.

Pequea-rosa. Rosarothe P. Bauholz.'A pocynaceae.

Peque-tanha. Vide Paó setim.

Pequi, Pequem, Pequehy, Piqui. Carycar brasiliense Camb. Rhizobalanaceae.

Pequi, Pekea in Parà. Carycar butyrosum Willd. Rhizobalanaceæ.

Pequi amarello. Gelbe P. Vide Arrore de manteiga.

Pequia. Vide Pequi in Parà.

Pequia a marella. Gelbe P. )

Pequia assù. Grosse P. f Vide Pequia de folha larga.

Pequia banana. Bananen P. Vide Pequi.

Pequia café, Pitia café. Kaffee P. Casearia dentata Eichl.

Flacourtiaceae.

Pequia de follha larga. Breitblättrige P. Aspidospermum sessili-

florum Fr. Allem. Apocynaceae.

Pequia marfim. Elfenbein P. Vide Paó setim.

Pequia-rana. Falsche P. Bombax globosum Aubl. Bombaceae.

In Alagoas Caryocar glabrum Pers. var. edule Wittm. Rhizobalanaceae.

In Amazonas Caryocar toxiferum Barb. Rodr. Rhizobalanaceae. 
Pequia da serra. (iebirgs l'. Vide Paó setim.

Pequi-lıy. Vide Vinhatico.

Pequim. Bauholz in den Nordstaaten.

Pera de advogado. Advocatenbirue. Vide Abacateiro. Pharm. Areh., 1898, p. 1.

Perdi\%. Rebhuhnkraut. Trixis brasiliensis DC. Compositae.

Perè. Tupyname für A vicennial nitida Jacq. Verbenareae.

Pereira, Peireiró. Aspidospermal pyrifolinm Mart. Apocynacear.

Alibertia edulis A. Rich. Rubiaceate.

lereirana branca. Weisse P. )

l'ereirana vermelha. Rothe I'. f Bauhölzer.

Pereirima, l'yririma. Cocos stagras Dr. Palmate.

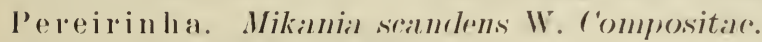

l'erikyty. Virle Jequirity.

l'erina. Vide Camna do mato mol Coqueiro tín.

Periparoba. Vide Pariparoba.

P'eririqueira. Bauholz in Bahia.

Periquiti. Benennumg in Alagoas fiir Jequirity.

Periquito. Vide Albarì.

P'eriquiteira. Vide Gurindiba.

Perlucho. Kirsche. Passifforal Parahlibensis Barb. Rodr. Passifloracrate.

Peroba. Birnenaihnliche Frucht. Aspidospermal perobal Fr. All. Apocynaceue.

In Campos vide Sobró.

In Parì Aspidosperma - - Aporynaceale.

In Pernambuco Tecomal —? Bignoniaceale.

Peroba assí. Grosse P. Aspirlospermal polyneuron Miill. Arg. Apocynacease.

Peroba branca. Weisse I'. Salpota gonocalpal Mart. et Eichl. Salpotaceae.

Peroba do eampo. Steppen I'. Sweetia elegalns Bth. C'aesalpiniacea. P'eroba mirim. Klene I'. Sweetia brachystachya Bth. Caesalpinialceap.

P'eroba parcia. Braune I'.

Peroba rajada. Gestreifte P. $\{$ Noch nicht bestimmte Bauhölzer. Peroba revessa. Knorrige P.

Peroba rosa. Rosarothe P. Chrysophyllum. Sapotaceae.

Perobinha. Vide Peroba mirim, auch Peroba do campo. 
I'erobinlia do campo. Vide Peroba do campo.

Perpetua. Telanthera brasiliana Moq. Amaranthaceae. Ph. Rdsch., 189:5, pp. 89, 90. Vide Paratudo (Gomphrena).

Perpetua do campo. Gomphrena Pohlii Moq. Amaranthaceae. Ph. Rdsch., 1895, pp. 89, 90.

Borreria scabioides Cham. et Schlecht. Rubiaceae.

P'erpetua dos jardins. Gomphrena globosa L. Amaranthaceae. Perpetua do mato. Telanthera ramosissima Moq. Amaranthaceae. Perrexil. Falsche Petersilie. Vide Amor crucido. Ph. Rdsch., 1896, p. 156 .

I'errexy. Heilpflanze in Alagoas.

Persicaria. Heilpflanze in Alagoas.

Persicaria. Vide Herva de Cicho.

I'eruci. 'Tupyname fïr I'erama hirsutid Aubl. Rubiaceae.

P'etituiba. Schiffsbauholz in Ceari.

Petumarana, Pitumarana. Tupybenennung fïr Genciana (Lisianthus).

Petum, Petume. Indianername für Fumo (Tabak).

Pety. 'Tupybenennung tïr Fumo de caboclo und Fumo bravo.

Peuva. Tecoma speciosa I)C. Bignomiaceate.

l'euva amarella. Gelbe I'. \} Varietiaten.

Peuva rosa. Rosarothe I'.

Piaca. Crudya obliqua Grieseb. Papilionaceae.

Piaca pelluda. Haarige P. C'ruclya pubescens Spruc. Mss. Papilionaceae.

Pião. Kreisel, wird in Parà .Jatropha rurras L. benannt. Ph. Rdsch., 1889 , pp. $111,261$.

Piassaba. Attalea funifera Mart. Palmae. Plı. Rdsch., 1889, p. 134.

In Amazonas Leopoldinia piassaba Wallace Palmae.

In Piauhy Arhignea Eichleri Dr. Palmae.

Piassaba brava. Wilde P'. Barcella oclora Tr. Palmae. Ph. Rdsch., 1889, p. 134 .

Piassaba fina. Feine I'. Orbignea racenosal I)r. P'almae. Ph. Rdsch., 1889, p. 1:34.

Picolıonha, Pikahim, Pukahim. Indianische Benennung, aus welcher das corrumpirte Wort Ipecacuanha entstanden.

Picao. Stachelkraut. Bidens pilosus L.; Bidens graveolens Mart. Compositae. Arch. d. Ph., 102. p. 166. 
Picao da praia. Kiisten St. Acanthospermum xanthioirles Dr:; Melampodium divalicatmm I)(: Compositae.

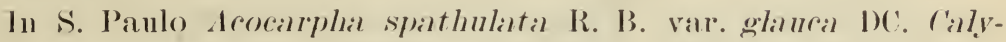
ceraceile.

Picao do tropeiro. Gomphrenal paniculatil Moq. Amaranthaceare. Ph. Rolsch.. 189.), p. 89.

Pichia, Piehúa. Euphorloia portularcoinles Spreng. Enphorliaceit. Pichuna. Schwarzbanm. Engenia gomeratal Spreng. Myrtaceate. Pichurim, Pechury, Puchury, Pexurim, Piexiri. Nectandral pnchnry maior Nees. Lanniareale.

Pichurim bravo. Wilke P.

Pichurim bastardo. Bastard P. C Lanrareale. Ph. Arch., 1898, Pichurim mirim. Kleine P. $\mid$ pp. 22, 28.

Piclurim-rana. Falsche P'. Nectamlra limbata Nees. Lanracoae. Pigni, Piqui. Evolvulvus daphnoides Mor. Convolvulaceae.

Pijericú, Pijericum, Pegrecou. Xylopia sericea St. Hil. Anonaceae. Ber. d. d. ph. Ges., 1897, p. 46.).

Pikia. Sickingia P'ikia Schum. Rubiareale.

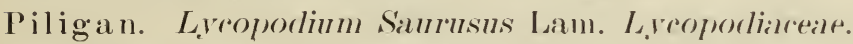

Pimbá. Vide Mangue verdadeira.

Pimenta. Allgemeine Benennung der Capsicumarten.

Pimenta do Par.i. Parapfeffer. Vide Agrião de Parà.

Pimenta d'agua. Wasserpfeffer. Polygonum acre HBKth. var. aquatile Meissn. Polygoniceae.

Pimenta de America. Vide Aroeira da capoeira. Ph. Rdsch., 1891, p. э).

Pimenta apúa. Capsirum bilveatum L. var. dichotomnn Vell. Solanaceae.

Pimenta do ar. Luftpfeffer. Piperomia repens HBKth. P'iperacer. Pimenta do cheiro. Wohlrechender P'f. ('apsicum frutescens Willd. var. odoriferum Vell. Solanaceate.

Pimenta chifre de viado. Vide Pimenta apúa.

Pimenta cobre cipo. Lianenschlangen Pf. Artanthe eximia Miq. Piperaceae. Ph. Risch., 1894, p. $2+2$.

Pimenta comarim, Cum a rim, Comar y. Tupy corrumpirt aus ciu mirim-kleine Frucht. Capsicum frutescens Willd. var. comarim Vell. Solanareae.

Pimenta Coroados. Vide Craveira da terra. 
Pimenta da costa. Afrikanischer Pf. Xylopia fintesrens Aubl. Anonacere.

Pimenta comprida. Langer Pf. Artanthe raurlata Miq. Piperacex. Ph. Rdsch., 1894, p. 241.

Pimenta fundo da garrafa. Flaschenboden Pf. (zufolge der

Form). Capsicum annunm L. var. Axi Vellos. Solanareae.

Pimenta fundo de lima. Muschelschalen Pí. Capsicum annunm

L. virr. grossum Sendt. Solanaceae.

Pimenta de galinhas. Hühnerpfeffer. Solanum nigrnm L. var. angulosim Sendt. Solanaceae. Vide auch Aguara quya.

Pimenta grande. Grosser Pf. C'apsicum annumm L. var. longum Sendt. Solanaceite.

Pimenta dos indios. Indianerpfeffer. Vide N'handí und Pijericú. Pimenta de macaco. Affenpfeffer. Cleome psoraleatolia DC:

Capparidaceae. Ber. d. d. ph. Ges., 1898, p. 4t. Auch Pijericú. Pimenta malagueta. Brennender Pfeffer (Afrikanisches Wort).

Capsicum frutescens Willd. var. malagueta Maregr. Solanaceae.

Pimenta malagueta a narella. Gelber brenn. P’. C'apsicum

barcatum L. var. quiva Piso. Solanareae.

Pimenta do mato. Willer Pfeffer. C'apsirnm campulopodium Sendt.

Solanaceae. Vide auch fructo do moreega. Ph. Rdsch., 1894, p. 286.

Pimenta miuda. Kleiner P’feffer. Vide Páo de mulata.

Pimenta ovos. Eierpfeffer. (apsicum ovatum DC. Solanaceae.

Pimenta pacoim. Vide Pimentão.

Pimenta de peixe. Fischpfeffer. Vide Pimenta comarim.

Pimenta pica de macaco. Affenzengungsgliedpfeffer. C'alpsicum frutescens Willd. var. harratum Vellos.

Pimenta pitanga. Pitangapfeffer. Capsicnm Milleri R. et Sch. Solanareae.

Pimenta. do rato. Rattenpfeffer. Solanenm oleracenm Rich.

Solanaceae.

Pimenta do reino. Piper nigrum L. Piperaceae.

Pimenta saropó. Cocosnusspfeffer. C'apsicum bacratum L. var. saropo Sob. Solanaceae.

Pimenta de sertao. Wüstenpfefier. Xylopia grandifloral St. Hil. Anonareae. Ber. d. d. ph. Ges., 1897, 1. 468.

Pimenta da terra. Einheimischer Pf. Vide Pimentão comprido und Pijericí.

Pimenta umbigo de tainha. Vide Pimenta de peixe. 
Pimenta uriquena. Sehr kleine Friichte. Var. von Pimenta comarim.

Pimentao. (irosser I'leffer. Fipsicum ammum I. Solanaceas.

Pimentao compriclo. Langer grosser ['f. C'alsicum annumm L. var. silvestre Vellos. Solillaceae.

I'imentao manso. Milder grosser Pf. C'apsicum annumm L. var. cordiforme Sendl. Solinareate.

Pimentao do mato. Cirosser wilder I'f. Amijona guianensis Aubl. liubiaceale.

Pinenteira da terra. Einheimischer Pfefferbanm. Vide Craveira da terra.

Pimenteira do mato virgen. Vorzïgliches Bauhol\%. Fragara? Rutilear.

Pimentinlıa. Kleinel P’f. C'apsicum microrarpum I)C: ('apsicum Hexuosum Sendt. Solanireate.

Pinacuns. Tupybenennung fïr Körbchen aus Palmenblattfasern.

Pindaiba, Pinclayba. Cormupirt des T'upyortes Pinda-kyba. Angelrutle. Vide Ibira.

Pindaiba do brejo. Sumpf I'. Krlopia emarginata Mart.

Pindaiba de carador. Jaiger P. Guatterial villosissima st. Hil.

Pindaiba preta. Schwarze P. Guatterial nigrescens Mart.

Pindaiba urary. Gift P'. Fuatteria veneficiormm Mart.

P’inau-juqui ŕı́. Vỉle C'urı́ı̂.

Pincel. Pinselpflanze. Emilia Himme; Cass. C'ompositap. Gartenpflanze.

P’indabuna. Vicle ('óijericú.

Pindóba, Pindóva. Corrumpirt von Tupy. Pinda-óva, Blatt für Angelruthe. Attileia rompta Mart. Pialmale.

In Alagoas vide r'oqueiro c'atolé.

In Goyaz Orbignea Eichleri Dr. Palmae.

In Rio de Janeiro vide Buri. Ph. Rdsch., 1888, p. 110.

In Matto Grosso Oenocarpus discolor Barb. Rodr. Palmae.

P'indobinha. (Pindo in Guaranisprache heisst Palme, inha portug. klein.) Geonoma ohapadensis Barb. Rodr. Palmae.

Pingo-pingó. Vide Morango do campo.

Pinguariba. Vide Páo Pereira.

Pinguem, I'inguin. Bromelia Pingnin L. Bromeliaceate. Ph. Rdsch., 1895, p. $2: 37$.

P'inguin da costa. Kïsten P. Bromelia Binoti E. Morr. Bromeliaceap Ph. Rdsch., 189\%, p. 237. 
Pinha. Tannenzapfen. Vide Fructo de Condessa. Ber. d. d. ph. Ges., 1897 , p. 457.

Pinha de brejo. Vide Canella de brejo. Ber. d. d. ph. Ges., 1896, p. 157 .

Pinlaao. Zirbelnuss. Vide Embiú bravo.

Pinhao bravo. Wilde \%. Jatropha Pohliana var. mollissima Miill. Arg. Euphorbiaceae.

Pinhao da cerca. Umzäumungs 7. ) Jatrophal C'urcas L. EnPinhao da purga. Purgir \%. $\quad$ f phorbiaceae.

Pinhao roxo. Violettrothe 7 . In Bahia vide Medicineiro.

Pinheirinha. Kleines Fichtchen. Vide Froco.

Pinheirinha d'agua. Wasserfichtchen. Myriophyllum brasiliense Camb. Haloragiaceae.

Pinheiro. Tanne. Araucaria brasiliana A. Rech. Coniferae. Ph. Rdsch., 1893, p. 133 ; Arch. d. Ph., 122, p. 219.

Pinheiro branco. Weisse T. | Varietïten, zufolge der Farbe Pinheiro vermelho. Rothe T. $\int$ des Holzes.

Pinheiro manso. Pinus pinea I. Coniferae. Kultirirt.

Pino. Brennkraut. Vide Quemadeira (Cnidosculus).

Pino-guassí. Vide Mamoeira.

Pintado. Gefleckt. Vide Angelim coro.

Piolho, Pioya. Vide Paó de lagarto.

Piolho de urubù. Vide Paó Brasil.

Piperioca. Cyperus sanguineo-fuscus Lindl. et Nees. Cyperaceap. Ph. Rdsch., 1894, p. 187.

Piperona. Vide Betys.

Pipi, Pepi. Petiveria alliacea L.

Petiveria hexaglorhen Fish. et Mey. Phrtolareaceae. Ph. Ridsch., 1895, p. 216.

Pipiri, Peripiri. Sumpfrohr (Tupy). Echinoschoenus sparganoirles Lindl. Rhynchosporaceae.

Piрово́. In siedendem Fett geplatzter Mais. Speise.

Piqui, Piquia, Piquihy. Caryocar brasiliensis Camb. Ternstroemiaceae.

In S. Paulo Carvocar Mrasiliensis Camb. var. planifolinm Ternstroemiaceae.

Piracaúba, Piracú-úba. Fischbaum. Vide Cuịumary.

Piracurú. Heilpflanze in Parà. 
Piraguaia, Piragaia, Pirigaya, P’irgual'á. Archicta sallutarlis St. Hil. Violareale. Arch. (1. Plı., 97, p. 271 ; Ber. d. d. ph. Ges., 1897, p. 97.

Piranga. In Tupy roth. Virle (arajúru. (Arabidara.)

Pirao. Dicker Brei von Mandioceamehl.

Piraútuxy. Bauhol\% in Parì.

P’iraví. Vide l’aí rei.

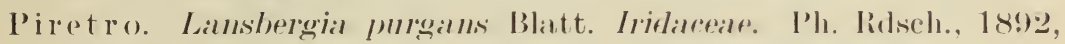
p. 1333.

Piri. Sumptrohr. Vide Junes.

Piriguao, Pirijao. Vide P'upunha.

Piriquiteira. Sittigbanm. Bauhol\% in Mlagoas.

Pirirema. Vide Pupunha de poreo.

Piriri. Malnea occillentalis var. gemnina Miill. Arg. Euphorhiarsate. Piririma. Vide Jatá.

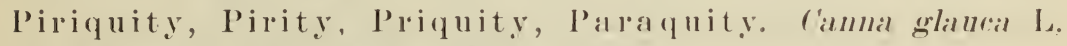
C'annilceae. Ph. Rdsch., 1893, p. 257.

l’ipiquity vermelho, Piriquitó. C'anna demulatal Rose. C'annaceae. Ph. Rdsch., 189:3, p. 257.

Pirur íca. 'Tupybenemung fïr Canjica.

Pissando, Pissandí. Diplothemium campestre Mart. var. Orbiygnii Dr. Palmale. Ph. Rdsch., 1889, p. 111.

P’ita. Vide Piteira.

Pitaica. Vide Tachi piqueno (Swartzia).

Pitaicica. Baulolz in Parà.

Pitanga. Von der Tupysprache. Yha-Baum, pitanga-rothe Frucht. Pitangueira. Benennung des Baumes. Stenocalyx Michelii Bg. Myrticeae.

In Minas S enocalyx pitanga Bg. Myrtaceae.

In Rio Grande do Sul Stenocalyx dasyblastus Bg.; Aulomytria bombycina Bg. Myrtaceae.

In Santa Catharina Stenocalyx sulratus Bg. Myrtaceate.

l'itangueira da serra. Gebirgs P. Stenocalyx sulcatus Bg. Myrtaceae.

Pitanga amarella. Varietąt von Stenocalyx Michelii Bg. Mvrtaceae.

Pitanga branca do mato. Weisse Waldpitanga. Bauholz. An Dr. v. Martius, Herbariumexemplar No. 317 gesandt. 
l'it anga de cachorro. Hundspitanga. Calvptrantes tubereulata Bg. Mvrtaceae.

In Minas Calyptrantes obscura Bg. Mrrtaceat.

In Rio de Janeiro Calyptrantes obscura var. Huminensis Bg. Mytaceae.

Pitanga do mato. Wald P. Gomidesia .Jacquiniana Bg. Mvrtilceate.

In Rio de Janeiro Stenocalyx ligustrinus Bg. Myrtaceate.

In Rio Grande do sul Gomidesia Sellowiand Bg. Myrtaceae.

Pitanga do mato virgem. Urwald P. Aulomyrcia pulchra Bg. Myrtaceae.

P'itanga miuda. Kleine I'. Aulom.rreia rubella Bg. Myrtaceae.

l'itanga pimenta. I'feffer P'. Mvrciaria longipes Bg. Myrtaceae. P'itanga preta. Schwarze P. Aulomvreia obovata Bg. Mvrtaceate. Pitanga vermellı. Stenocalyx Michelii Bg. Myrtaceae.

Pitanga-ïba, Pitangatuba. Plyyllocalyx edulis Bg. Myrtaceae. P’itar. Carludovica divergens Dr. Cyclanthaceate. Ph. Rdsch., 189:3, p. $13 . \overline{5}$.

Pitaya. Heilpflanze in Alagoas. C'ucurbitaceae.

Pitay-ya. Virle Jumbela.

Piteira. Fonrcroya gigantea Vent. Igaveat. Ph. Rdsch., 1892, p. $16: 3$.

Piteira assì. Vide Caraquatá-assì.

Pitimujú, Putumuyà. Nutz- und Tischlerholz in Mlagoas.

Pitoma nach Marcerrav. Sapindaceae?

Pitomba. Frucht. Pitombeira. Baum. Talisia escrnlenta Rarllk. Sapindaceate. Ber. d. d. ph. Ges., 1902, p. 107.

In Bahia vide Curuiri.

In Parà Simaba guyanensis Engl. Simarubaceate.

In Pernambuco vide Pitomba do sertao.

l’itomba assì. Talisia intermerlia Radlk. Salpindaceate. Ber. d. d. ph. Ges., 1902, p. 108.

I'itomba brava. Wilde l'. Tralisia cerasina Radlk.; Talisia acutifolial Radlk.; Talisial cupularis Radlk. Sapindaceate. Ber.d. d. ph. Ges., 1902, p. 108.

Pitomba de leite. Milch P. Vide Pombeba.

Pitomba do mato. Wald P' I Vide Pitomba assì.
Pitomba-rana. Falsche P. 
Pitomba do sertao. Wüsten P. Matayba heterophylla Radlk.

Sapindaceae. Ber. d. d. ph. Ges., 1902, p. 111.

Pitombó. Phvllocalyx strictus Bg. Myrtaceae.

Pitombó miudo. Kéleine P. Eugenia Luschnathianus Bg. Myrtaceae.

Pitumarana. Vide Petumarana.

Pitumbá. Casearia macrophylla Vahl. Flacourtiaceae. Ber. d. d. ph. Ges., 1899, p. 172.

Piúva. Vide Ipé.

Pixirica. Lencothoe breviflora Meissn. Melastomaceae.

In Rio de Janeiro vide Mexerica.

In S. Paulo Leandra scabra DC. Melastomaceae.

Pluma de Persea. Persische Feder. Trixis verbasiformis Less. Compositae.

Plumerilho. Vide Quebra foice.

Poaya. Ipecacuanha. Nach Tupy-schlechte oder schlimme Wurzel.

Viele Pflanzen haben bei den Indianern diese Benennung, von welchen der Genuss der Wurzeln Uebelkeit verursacht. Das Wort ist abgekürzt von capo-Wurzel, und aina-schlecht, oder pó-schlimm.

Psychotria ipecacuanha Müll. Arg. Rubiaceae.

Poaya arvore. Baum I. Psychotria pleiocephala Müll. Arg. Rubiaceae.

Poaya bastarda. Falsche I. Corynostilis hybanthus Mart. et Zucc.

Violaceae. Ber. d. d. ph. Ges., 1897, p. 97.

Poaya batata. Knollen I. Dipteracanthus tuberosus N. ab. E. Acanthaceae.

Poaya branca. Weisse I. Richardsonia scalora L. Rubiaceae. Vide Itubú.

Hybanthus brevicanlis Taub.

In Bahia Hybanthus circaeoides Taub.

In Rio Grande do Sul Hybanthus albus Taub.

Hybanthus bicolor Taub. Violaceae. Ber. d. d. ph. Ges., 1897, pp. $97-102$.

In Rio de Janeiro Richardsonia brasiliensis Gomez. Rubiaceae.

Diodia saponariifolia Schum. Rubiaceae.

Diodia latifolia Schum. Rubiaceae.

In S. Paulo Richardsonia rosea St. Hil. Rubiaceae.

Poaya branca do campo. Weisse Steppen I. Hybanthus glutinosum Vent. Vide Poaya branca de Bahia. 
In Minas vide Podya cabelluda und Manettia cordifolia Márt. Rubiaceae.

I'oaya brava. Wilde I. Dipterocanthus tomentosus N. ab. E. Acanthaceae.

Poaya de brejo. Sumpf I. Machaonia brasiliensis Cham. et Schl. Rubiaceae.

Poay a cabelluda. Haarige I. Hybanthus rillosissimus Taub.

Violaceae.

Poaya do ca in po. Steppen I. Liodia poaya DC. Rubiaceae.

Diodia polymorpha Cham. et Schl. Rubiaceae.

Borjania linearis Duc. Asclepiadaceae.

Hybanthus poaya Taub. Violaceae.

Borreria poaya DC. Rubiaceae.

Keithea denudata Bth. Labiatae.

Polygala angulata DC. Polygalaceae.

Poaya de capoeira. Hybanthus setigerum Taul. Violaceae.

Poaya comprida. Borreria emetica Mart. Rubiaceae.

Poaya legitima. Aechte I. I

Poaya do mato. Wald I. Y V.de Puaya.

Poaya da praia. Küsten I. Hybanthus ipecacuanha Taub. Violaceae.

Borreria ferruginea DC. Rubiaceae.

Borreria capitata DC. Rubiaceae. •

Machaonia brasiliensis IV. Rubiaceae.

Poaya preta. Schwarze I. Vicle Poaya.

Poaya da serra. Hybanthus communis Taub. Violaceae.

Poay a timbó. Gift I. Psychotria Peckoltiana Miill. Arg. Rubiacere.

Poaya verdadeira. Vide Poaya.

Poaya a tôa. Nutzlose I. Borreria verticillata G. F. W. Meyer. Rubiaceae.

Poay vermelha. Rothe I. Manettia luteo-rubra Bth. Rubiacere. Pobúra. Vide Angelim.

Poejó. Polei. Cunila microcepha Bth. Labiatae.

Poejo bravo. Wilde P. Glechon caparannensis Taub. Labiatae.

Poejó do brejo. Sumpf P. Vide Acarizoba.

Poejó do campo. Steppen P. Keithia denudata Bth.; Cunila

spicata Bth. Labiatae. Arch. d. Ph., 1857, 91, p. 239.

Poejó da praia. Küsten P. Vide Poejó bravo.

Pojamarióba. Tupyname für Herva de bicho. 
Polvilho. Volksname für Stärkemehl.

Polygala da terra. Einheimische P. Polygala fimbriata Bennet. Polygalaceate.

Ponceta. Euphorbia pulcherrima Willd. Euphorbiaceae. Arch. d. Ph., 94, p. 363; 99, p. 291.

Poraque-iba. In Tupy ist Poraque-electrischer Aal, iba-Baum.

Poraqueiba guianensis Aubl. Icacinaceae.

Porov-assù. Grosse P. | Indianische Benennung für Perobinha Porov-mirim. Kleiner P. $)$ do campo.

Porocó. Vide Broca.

Porongó. Varietät der $\Lambda$ bobora carneira.

Póróróca, Páróróca. Comumpirt aus der Guarani-Sprache, póróróg-mit Geräusch platzen, wenn ein frisches Blatt erhitzt wird. Vide Orelha de burro.

Pórórom. Unreife Wassermelone (Melancia).

Portulacca da terra. Vide Beldroega. Ph. Rdsch., 1896, p. 155. Pós de mico. Affenpulver. Mucuna pruriens DC. Papilionaceàe.

Pós de Santa Anna. Heiliges Annenpulver. Vide Massarandíba de S. Paulo.

Poterio. Clitoria cujabensis Bth. Papilionaceae.

Potincoba. Tupyname für Herva Pulgeria.

Potúmujú, Putúmujú. Vide Arariba.

Pqa. Pekea Couroupita Juss. Myrtaceae.

Pracachi, Procachi. Pentaclethra filamentosa Kth. Mimosacea. Pracari. Vide Vigueiró.

Pracuúba. Bauholz in Parì.

Pragúa. Heteropteris panciflora Juss. Malpighiaceae.

Pragúa do campo. Heteropteris seringaefolia Grieseb. Malpighiaceae.

Prajá. Confect in S. Paulo von eingedicktem Zuckerrohrsaft mit Eigelb.

Préha-caa. Tupyname für Herva préa.

Primavera. Frïhlingsblume. Ipomea quamochit L. Convolvulacex. Primavera da serra. Gebirgs F. Ipomoa coccinea [. Convolvulaceae.

Prituiba. Heilpflanze in Amazonas.

Ptii. Tupyname für Cipo alho.

Púba. Eingeweichte und gegohrne Mandioccaaypimrübe, aus welcher Torte und vegetabilischer Käse bereitet wird. 
Puca. Flohsamenkraut. Vitis bricaspis Bak. Ampelidaceae.

Púca brava. Wildes F. Heilmittel in Ceàrà.

Púca do mato. Vitis salutaris Bak. Ampelidaceae.

Púcahy vide Mundahir.

Pui-hem-assú, Tupyname für Pimentaõ.

Punú mirim. Kleine Nepel. Urera aurantiaca Wedd. Urticaceae.

Pupunha. Frucht. Guillielma speciosa Mart. Palmae.

Pupunheiro. Baum. $\quad$ Pharm. Rdsch., 1888, p. 204.

Pupunheiro sem espinha. Stachelloser P. Guillielona speciosa

Mart. var. mitis Dr. Palmae.

Pupunheiro marajá. Guillielona speciosa Mart. var. flava Barb.

Rodr. Palmae.

In Amazonas var. coccinea Barb. Rodr. Palmae.

Pupunheiro de Porco. Schweins P. Cocos Inajai Trl. Palmae. Ph. Rdsch., 1889, p. 92.

Cocos speciosa Barb. Rodr. Palmae. Ph. Rdsch., 1889, p. 92.

Pupunha-rana. Cocos Chavesiana Barb. Rodr. Palmae.

Pureza. Iucca gloriosa L. Liliaceae. Kultivirt.

Purga de A maro Leite. Vide Jalapa de S. Paulo.

Purga de caboclo. Indianerpurgans. Vide Purga de gentia in Minas.

Purga do campo. Steppenpurgans. Benennung für Purga de veado, Itubú, Espelina, Poaia branca (Jonidium) do campo und Rosa do campo.

Purga de carijó. Perianthopodus Weddelii Naud. Cucurbitaceae. Purga de cavallo. Pferdelaxans. Ipomoea. Silvano Chois. Convolvulaceae. Vide auch Anda-assù.

Purga de Cayapó. Cayaponia pilosa Cogn. Cucurbitaceae.

In S. Paulo Cayaponia ternato Cogn. Cucurbitaceae.

Purga de cereja. Hirsenpurganz. Cayaponia cordifolia Cogn.

Cucurbitaceae.

Purga de gentio. Indianerpurganz. Cayaponia hirsuta Cogn. Cucurbitaceae.

In Minas Cayaponia caboclo Mart. Cucurbitaceae.

In Rio de Janeiro Cayaponia fluminensis Cogn. Cucurbitaceae.

Auch Anda-assú und Cereja do mato haben diese Benennung.

Purga de Joa õ Paes. Volksname für Caa-ataya.

In S. Paulo heisst es Bucha. 
Purga de leite. Milchpurganz. Excalcaria agallocha Tr. $\Lambda 11$. Euphorbiaceae.

Purga de marinheiro. Matrosenpurganz. Vide Gité

Purga do pastor. Hirtenpurganz. Diplarlenia polvmırpha Miill.

Arg. Apocynaceae.

Purga dos paulistas. Vide Bucha dos paulistas.

Purga de quatro patacas. Allemanda violacea Gardn. et Field. Apocvnaceae.

Purga de veado. Rehpurganz. Iipladenia illustris Müll. Arg. Apocynaceae.

Vide auch Apanha seùa.

Purgueira. Abführbaum, wird in Ceara fïr Pinhaó de purga benannt.

Puruhy. Bunchosia fluminensis Gr. Malpighiaceae.

In Rio de Janeiro vide Azucena do mato.

Purùi. Alibertia edulis Rich. Rubiaceae.

Puranan. Palme in Alagoas.

Purúríca. Vide Mororóbranco.

Purúrucò. Eingeweichte, zerreibbare Maiskörner.

Putúrema. Bauholz in Parà.

Pyxerica. Auch Mnianga pixerica. Clidemia lirta D. C. Melastomaceae.

Quabiroba de cachorro. Vide Quabiroba de cachorro.

Quaparaiva. Lecythis Pohlii Bg. Lecythidaceae. Ber, d. Ph. G. 1902.

Quapoya. Vide Pana-panà-ré.

Quaronta feridas. Vierzig Wunden. Vide Espinha de Judeo.

Quaresma. Fastenkraut. Miconia tomentosa Naud. Melastomaceae. Quaresma amarella. Gelbes F. Cassia bacillaris L. Caesalpiniaceae.

Quaresminha. Kleines F. Vide Herva de Quaresma.

Quariròba. Vide Guabiróba.

Quaró. Vide Cipo de arco und Cùaró.

Quassia. Quassia amara L. Simambaceae. Ber. d. Ph. G., 1898, p. 427.

In Amazonas vide Caferana.

Quatilé. Lecythis Pisonis Camb. , Lecythidaceae. Ber. d. Ph. l.ecythis amazonum Mart. $\} \quad$ G., 1902.

Quatro patacas. Vide Purga de 4 patacas. 
Quaxindúba. Vide Gamelleira.

Quaxindúba preta. Vide Quaxima preta.

Quebêbe. Süsse Speise von Abobora melaõ.

Quebra facas. Messerbrecher. Croton carycphyllus Bth. Euphorbiaceae.

Quebra foice. Sichelbrecher. Calliandra Tweedii Bth. Mimosaceae. Quebra machado. Axtbrecher. Vide Grundeuva.

Queci-mirim. Tupyname für Capsicum fiutescens Willd. Solanaceae. Quejadilho. Primula veris L. Primulaceae. Kultivirte Pflanze.

Queimadeira. Brennbaum. Jatropha oligandra Müll. Arg. Euphorbiaceae.

J. horrida Müll. Arg. Euphorbiaceae.

In Alagoas und Parà J. ferox Miill. Arg. Euphorbiaceae.

In Ceara J. urens var. genuina Müll. Arg. Euphorbiaceae.

In Minas J. urens var. Maregravii Müll. Arg. Euphorbiaceae.

In Rio de Janeiro J. urens var. neglecta Mïll. Arg. Euphorbiaceae. Arch. d. Ph., 1860, Bd. 102, p. 166.

Queimadeira cipó. Brennliane. Dalechampsea Peckoltiana Müll. Arg. Euphorbiaceae.

Quengá. Nationalspeise im Staate Bahia, von Fleisch, Quimgombó und Capsicum.

Quengó. Trinkschale von Cocosnuss.

Queraiba. Tupyname für Pá d'arco und Pavonia rosea Schl.

Malvaceae. Ber. d. Ph. G., 1900, p. 270.

Querepére. Bauholz in S. Paulo.

Quêrêquêxê. Indianerflöte von Taquararohr.

Queretá. Heilpflanze in S. Paulo.

Query branco. Weisse Q. Bauholz in Pernambuco.

Quiabó. Vide Quimgombó.

Quiabó bravo. Wild Q. Vide Carrapichinho. Ber. d. Ph. G., 1898, 282.

Quiabósinho do campo. Kleine Steppen Q. Periqueta aurea

Urb. Turneraceae.

Quiarte. Vide Cipó amarra gigante.

Quigába. Vide Paó de colher. (Tupyname).

Quillaya. Quillaya brasiliensis Mart. Rosaceae.

Quimbôa. Vide Betonica.

Quimbôa brava. Wilde Q. Mandirola multiflora Den. Gesneraceae. 
Quimbôa mansa. Wilde Q. Achimenes rupestris Hook Gesneraceae. Quimgombó, Quingombó. Abehnoschus esculentus Mey. Malvaceae. ( Quingombó chifre de veado ) Varietïten desselhen. Ber. d. Ph. Quingombó de gomos Quingombó de Maranlấ G., 1900 , p. 420 .

Quingombó de cheiro. Wohlriechende (2. Abeimoschus moschatus Medi. Malvaceae.

Quimgombó de espinha. Stachel G. Martynia lntea var. Nelsoniana Barb. Rodr. Martyniacear.

Quina. Chinarinde.

Quina bicolorado. Zweifarbige Chinarinde. Quina de S. Paulo. Quina branca. Weisse Ch., auch Chupa ferro. Eisensauger. Galipena jasminiffora Engl. lintaceae. Ber. d. Ph. G., 1899, p. 327. Quina brana do campo. Weisse Steppenchina. Vide Angostura do campo, p. 325.

Quina do Cabo frio. Coutarea hexandra var. Huminensis Schum. Rubiaceale.

Quina de Camamú. Diplodenia sancta Dl. Apocynaceale.

Quina do campo. Steppenchina, auch Quina branca. Strychnos Pseudoquina St. Hil. Loganiaceae. Arch. d. Ph., 1856, Bd. 85, p. 227.

Quina cipó. Lianenchina. Vide Cipó quina. Ph. Rdsch., 1893, p. 82 .

In Minas Strychnos Gardneriana A. Dl. Loganiaceae.

In Rio de Janeiro Strychnos triplinervis Mart. Loganiaceae. Quina cruzeira. Vide Cipó quina.

Quina de Cuiába. Ladenbergia cuyabensis Vel. Rubiaceae.

Remigia cuyabensis Wedd.

Quina falsa. Falsche China. Vide Chupa ferro.

Quina de folha larga. Vide Quina do Rio de Janeiro.

Quina de folha minda. Vide Quina do Cabo frio.

Quina de Goyaz. Vide Quina do Porto Alegre.

Quina de S. Joã̃. Macrocnemum grandiflorum Wedd. Rubiaceae. Quina de Manaos. Ladenbergia Lambertiana Vel. Rubiaceae. Quina do mato. Wilde China, auch Cascarilha falsa. Bathysia australis Hook. fil. Rubiaceae.

Bathysia floribundum Hook. fil. Rubiaceae.

In Minas Bathysia cuspidata Hook Fil. Rubiaceae.

In S. Paulo. Alseis floribunda Schott. Rubiaceae. 
Quina do mato do Rio Grande do Sul. Cestrum pseudoquina Mart. Solanaceae.

Quina do mato da serra. Wilde Gebirgschina. Bathysia gymnocarpa Hook. Fil. Rubiaceae.

Quina officinal. Officinelle China. Chinchona officinalis I. Rubiaceae. Kultivirt auf dem Orgelgebirge.

Cinchona Carabayensis Wedd.

Quina do Pará. Vide Paó de lagarto legitimo.

Quina pavana. Gustavia augusto L. var. brasiliensis Bg. Lecythidaceae. Ber. d. Ph. G. 1902

Quina de Pernambuco. Coutarea hexandra var. speciosa Schum. Rubiaceae.

Quina de Piauhy. C. hexandra Schum. Rubiaceae:

Quina dos pobres. Armenchina. Basanacantha annae Schum. Rubiaceae.

Quina do Porto Alegre. Rustia formosa Kl. Rubiaceae. Quina do Remigio. Remigia ferruginea Dl. Rubiaceae.

Quina do Rio Grande do Sul. Discaria febrifuga Mart. Rhamnaceae.

Quina do Rio de Janeiro. Vide Quina vermelha. Rubiaceae. Quina do Rio Negro. Remigia firmula Wedd. Rubiaceae.

R. Bergeriana Wedd. Rubiaceae.

$R$. macrocnemia Wedd. Rubiaceae.

Quina do S. Paulo. Solanum pseudoquina St. Hil. Solanaceae.

Quina da serra. Gebirgschina. Remigia Hilarii Dl. Vide Quina do Remigio.

Quina serrado. Vide Quina do campo (Strychnos).

Quina de tres folhas brancas. China mit 3 weissen Blättern.

Vide Quina branca. (Galipea).

Quina vermelha. Rothe China. Ladenbergia hexandra Kl. Rubiaceae.

Quinquefolia. Fünfblatt. Potentilla reptans L. Rosaceae. Kultivirt.

Quinquina. Galipea jasminiflora var. febrifuga Engl. Rutaceae. Ber. d. Ph. G., 1899, p. 327.

Quioquió. Tupyname für Coqueiro Dendé. Ph. Rdsch., 1889, p. 165.

Quipá. Echinocactus pachycentrus Lehm. Cactaceae. 
Quiquôa und Quinnputí. Indianische Benennump fïr Batata doce.

Quiri, Quiriùri, (2uirari, C'uirùriri. Jyrtus mmbiliciltal Ciumb. Myrticease. Vide Frei Jorge.

Quitandé. Wird in Bahia Feịaõ mindo benamnt.

Quitrive. Eine indianische Benenumg fïr Biriti.

(2uiti. Paó de sabaio. Ber. d. Ph. G., 1902, p. 10:3.

Quitio. Hyptis lencocephialil Mart. Labiatie.

(2uitoco. I'lucheal quitor 1)1. C'ompositale.

(2uixiba. Bumalia sertornm Fr. All. Silpotalerile. Vide Rompe gibaī.

Quixiúme. C'lusquea anpitulifer: Trin. Gramintale. I'h. Rilsch., 1894, p. 169.

Quiya, Quiynha, Kiynha. Tupyname fïr Pimentao manso.

Quiуa-apía. 'Tupy fïr P'inenta apía.

Quiya-assii. Tupy fïr Pimenta grande.

Quiyaqui. Tupy fïr Pimenta Comarim.

(quiya-uca. Tupy für Pimentaí.

Rabaca, Rabaza. Wassereppich. Leptoclininm trichotommm Bth. C'ompositre.

Rabanete. Radieschen. Raphilnus sativus L. var. radiculil.

Rabaõ. Sclwwarzer Rettig. R. siltivus L. var niger.

Rabai de cavallo. Meerrettig. Naturtium ar- auf d. Orgelmoracia F. Schulz.

gebirge.

Rabo de Arará. Araráschwanz. Tupyname ist Arará-petui Parkia pendula Bth. Minnosaceale.

In Parà: Norantea brasiliensis Chois. Maregraviaceale. Ber. d. Ph. G., 1901, p. 94.

Rabo de boî. Ochsenschwanz. Lirianthus asper Nees var. bräsilianna Hack Gramineale.

Rabo de Cugió. Brïllaffenschwanz. Alsophilat ferox Prest. Cyaltheaceate.

In Rio de .Janeiro: Machatrinm gracile Bth. Palpilionaceap.

Rabo de cavallo. Pferdeschwanz. Krannhia Horibundil Taub. Papilionaceate.

Rabo de cavallo falso. Falscher P'ferdeschwanz vile dararanda branca. Gartenpflanze. 
Rabo de guariba. Affenschwanz. Hyptis pectinata Port. Labiatae. Rabo de Jacaré. Krokodilschwanz. Rihipsalis macrocarpa Mig. Cactaceae.

Rabo de macaco. Affenschwanz. Alsophila armata Presl. Cyatheaceae.

Rabo de porco. Schweineschwanz, auch Murcella. Dalechampia caparonioides var. genuina Müll. Arg. Euphorbiaceae.

Rabo de raposo. Fuchsschwanz. Panicum scandens Trin. Girimineae.

Rabo de rato. Rattenschwanz. ('anlarea atfinis St. Hil. Malpighiaceae.

Rabo de rojaó und roxaó. Vide Coaró bravo.

Rabo de tatú. Gürtelthierschwanz. Vide Sumaré.

Rabo de tucano. Pfefferfresserschwanz. Voch.rsia oppugnatia Warn. Vochysiaceae.

Rabugem. Räudenbaum. Platyniscium Blancheti Bth. P'appilionaceae.

Do sertaõ. Wiusten R. Cordial Sellowiana Cham. Boraginaceale. Ber. d. Ph. G., 1902, p. 131.

Rochador. Spaltbaum, ferner Envira und Guasima de Mangue. Hibiscus tiliaceus St. L. Ber. d. Ph. G., 1900, p. 419.

Rainha dos lagos. Seekönigin. Vide Mureré-yg.

Rainha de noite. Königin der Nacht. C'rreus graudiflorus Mill.

Cactaceae. Blume öfnet nur zur Nacht.

Raiz a morgoso. Bitterwurzel. Lisianthus alatus Aubl.

L. chelosoides L.

Coutoubea spicata Aubl.

Vide Céntaurea de mato.

Gentianaceale.

Ber. d. Ph. G., 1899,

p. 224 .

Raiz de Cabeira. Geiferwurzel. Amblianthera versicolor Mïll. Arg. Apocynaceae.

Raiz de brandaõ. Fackelwurzel. Excoecaria potamophila Miill.

Arg. Euphorbiaceate.

Raiz do Brasil. Vide Poaya (Ipecacuanha).

Raiz de carurú. Vide Carurú.

Raiz de chá. Theewurzel. Cordyline terminalis Engl. Liliacear'. Ph. Rdsch., 1893, p. 81. 
Raiz de cobra. Schlangenwnrml. Trixis ophiorhiza Gard. Compositate.

In Mavanhaõ heisst Gafan'wlo und Raiz de tiú.

Rai\% contra. Schlangenanıiılot. Bredermeyera florilumda Willd. l'olygalaceile.

Raiz de corvo. Rabenwurzol Cassia rugosal Don. Cáesalpiniaceare.

In S. Paulo Byrsonima ralliabilis Juss. Malpighiaceae. Heisst auch fructo de perdis. Jith!mhnfrucht.

Raiz de escamas. Schuppenwurzel. Virle Fel da terra (Loplophytum). Pl. Rdsch., 1s!).), p. 33.

Raiz de frade. Mönchwurzel. Vide ('aim:il do campo.

Raiz de Guiné. Guineawury. I'otireril allialleal L. Phytolaccareale.

Ph. Rdsch., 1895, p. 216.

Raiz de Jacaré. Vide Cafielinll. Ler. 1. I'lı. G., 1899, p. 226.

Raiz de .Joaó Costa. Vile Ririz milienoril.

Raiz de lagarto. Vide Hevir de lagirto (C. parvifolia).

Raiz de larangeira. Vide Jalilpió.

Raiz milagrosa. Wunderwmol. Inedermerera linrifolia $\mathrm{Kl}$. Polygalaceace.

Raiz mil homen. Vide Mil homen.

Raiz d'Ouro. Goldwurzel. Vide Paaya (Ipecaonauha).

Raiz de padre Salerno. Salerner Pfaffenwur-

zel. Gomphrena macrorhiza Mart.

Amaranthaceae.

G. macrocephala St. Hil.

Ph. Rdsch.,

Raiz para tudo. Wurzel fiir Alles. Vide Para-
tudo (Gomphrena).

Raiz Pipi. Vide Pipi.

Raiz preta. Schwarzwurzel. Chiococca brachiata R. et P. var.

densifolia Müll. Arg. Rubiaceae. Dieselbe Benennung haben auch:

Canica, Caninana und Raiz de corvo.

Raiz de quina. Chinawurel. Vide Cipoquina.

Raiz de quina branca. Weisse Chinawurzel. Vide Japercanga.

Raiz de sabaõ. Seifenwurzel. Vide Cajá murú.

Raiz de Sol. Sonnenwurzel. Aristolochia floribunda Lemais. Ari-

stolochiaceae. Ph. Rdsch., 1893, p. 181.

Raiz de teiú. Teiúdechsenwurzel. Vide Herva de lagarto in S.

Paulo.

Raiz de tiú. Schaumwurzel. Jatropha elliptica Miill. Arg:

Raiz de tyjú. Euphorbiaceae. 
Rairosa. Wiithend. Vide Tiborna in Minas.

Rama de prata. Silberzweig. Vide Cambaratúba.

Rama de vaqueiro. Hirtenpeitsche. Vide Pá-úna.

Ramalhete de mato. Waldblumenstrauss. Vide Paó mondè.

Ramallete da serra. Gebirgsblumenstrauss. Vide Jatúa-úba.

Rapadura. ' Zu einer festen Masse eingedickter rolıe '/uckersaft in

Backsteinform.

Raspas. Feine Mandioccarïben-Schnitzel, an der Sonne getrocknet,

gepulvert zu Gebäck etc. In den Nordstaaten heisst Apáras, die

Guaranaindianer benennen Typyraty.

Rassamalha, Ross omalha. Tolksname für Storax liquidus.

Rasteirinha, auch Córaçáosinha vide Mainibú.

Rasteira. Kriechpflanze. Monnina cardiocarpa St. Hil. Polygalaceae. Ratanlia. Krameria tomentosa St. Hil.

Ratanhia da terra. Krameria argentea Mart. Polygalaceae.

Ratimbó. Citharexylum myrianthum Cham. Verbenaceae.

Rebenta boi. Ochsenpflatzer. Vide Arrebenta boi.

Rebenta cavallo. Pferdepflatzer. Isotoma longiflora Presl. Lobe-

haceae, vide auch Arrebenta cavallo.

Redondilho und Rodonel. Triticum turgidnm Sims. Gramineae. Rego vide Cipo rego.

Relogio. Uhr. Vide Chá inglez.

Relogio de palma. Palmenuhr. Vide Malva brava.

Remedio de vaqueiro. Kuhhirtenarznei. Ocimum canum Sims.

Labiatae.

Repollo. Kopfkohl. Brassica oleracea L. var. capitata. Cruciferae. Ber. d. d. Ph. G., 1897, p. 286.

Reseda. Resedal. Lawsonia alba Lam. Lythraceae. Gartenpflanze. Reseda amarella. Gelbe R. Galphimia brasiliensis Juss. Malpighiaceae.

Reseda do campo. Steppen R. Bauholz in Matto Gropa.

Retirante in Ceara. Die Benennung für Picaõ da praio (Aconthospermum.

Rhuibarbo de campo. Steppenrhabarber. Lans-

bergia cathartica Kl. in S. Paulo heisst Batatinha Iridaceae,

do campo und Baririço amarello, vide Jonquilho, Ph. Rdsch.,

Rhuibarbo do charco. Pfützen R. Vide Pirebro. (1892,

Rhuibarbo de mato. Wilder R. Lansbergia cara-
casana DeVries. 
Riambí. Afrikanisches Wort, Negername fiir Pango().

Ricino. Virle Mamona.

Rinchao. Hederich. Sisymlrimm officinale Soop. Cruciferate. Ber.

1. P’h. G., Berlin, 1897, p. 286.

Rocú. Indianische Benemmung fïr I'rucú.

Roixinlıo. P’ortugiesischer Name fiir Guarúbú.

Romeira. Granatapfel. Pmnical granatum L. 1 Myristicacealf.

Romeira brava. Wilder G. Pnnical nana L. j kultivirt.

Romerilho. Kleiner Losmarin. Heterothalamms brunicires Less.

Compositale.

Rompe gibấ. Kleiderzerreisser. Bnmelia sintornm Mart. Salpotaceae.

Rosa branca. Weisse R. I Rosasetigeral Micli. Rosareae Officinell. Rosa brava. Wikle R. I Auf den Orgelgebirge, Unkraut.

Rosa de caclooro, Rosa handalho, Rosa mijona. Hundsrose.

Rubus rosatolius var. coronarins Sims. Rosalceale.

Rosa de caboclo. Indianerrose. Lingsdorfia hypogaleal Mart.

Balanophoreae. Ph. Rolsch., 189.), 34. Ktsch. d. Oestr. Ap. Ver. 1883, No. 26 .

Rosa de campo. Steppenrose. Dipladenia illustris val. tomentosal

Miill. Arg. Apocynaceae. Vide auch Malva de campo.

Rosa da India. Tagetes erecta L. Compositae. Kultivirt.

Rosa louca Rosa Paulista $\}$ Vide Papoula.

Rosario de jambó. Rosenkranzjanbö. Engenia raremosa Ol.

Myrtaceae.

Rosalina, Rorella. Röschen. Drosera villosa St. Hil. , Droseraceale, Rosalina de campo. Steppenröschen. Drosera Ber.d.Ph.G. communis St. Hil.

1897, p. 280.

Rosca. Schraube, Kringel, auch Guaxuma branca und Guaxuma do mato Helicteres muscosa Mast.

Rosca para mulas. Mauleselkringel, vide Embira do mato. Sterculiaceae, Arch. d. Ph., 18.56, Bd. 85, p. 220; Ber. d. Ph. (i., 1900 , p. 59.

Roseira do brejo. Sumpfrosenbaum. Vide Jasmin de brejo.

Roseiro do mato. Waldrosenbaum. Bougainvillea spectabilis

Willd, Nyctaginaceae. Ph. Rev., 1893, p. 53.

Roseirinha. Kleiner Rosenstranch. VileApotia-coraca (Beruardia). 
Roseta. Sporenrïidchen. Vide Roseira do mato.

Roseta (in Sta. Catharina). Acanthonychia laumosissima val. rosetta Rohrb. Caryophyllaceae. Ber. A. Ph. G. 1901, p. 211.

Roseta (in Pernambuco). Rhipsalis sarmentacea Otto et Dietr. Cactacear.

Roseta maritima $) \begin{array}{r}\text { Kïstensporen. Acicarphil spathnlata R. Br. } \\ \text { Calvceraceae. Ber. d. Ph. G., 1901, p. }\end{array}$ Roseta do praia | 96 , heisst auch Picaô do praia und farrapicho do praia.

Rosquinlı. Kleine Schraube. Vide Sacarolha do sertaõ.

Rouhamon. Strychnos Rouhamon Bth. Loganiaceae.

Rua o. Gummigutt. Tismia hrasiliensis Chois

Vismia decipiens Cham. et Schlechter. Viıle

Cád-opia.

R u a ö assú. Grosse G. Vismia Martiana Reich.

Guttiferae, Ber. d. Ph. G., 1897 , p. 235.

Ruivinha. Kleine Färberröthe. Relbunium noxicum,

Schuh. Belbunium hirtum Schum., heisst auch Ca- ( Rubiaceae.

angai. Vide auch Bacupari miudo (Coccocypsdum.)

Ruirinha do campo. Steppen F. Relbunium hirtum

var. camporum Śchum.

Ruivinha da serla. Gebirgs F. Relbuninm hypo-

carpum Hemsl var. indecornm schum.

liutin. Rotang. Desmoncus polyacanthos Mart. Palmae. Ph.

Ridsch. 1888, p. 265.

Sabina. Erythroxylon microphyllum St. Hil. Erythroxylaceae.

Saboarana. Falscher Seifenbaum. Bauholz in Staate Amazonas.

Saboeira , Seifenbaum. Sapindus saponaria L. Sapindacear.

Sabonete $\quad$ Ber. d. Ph. G., 1902, ए. 104.

Sabonia. Cestrum. Solanaceae.

Sabonete cipo. Gouania. Rhamnaceae.

śabueira. Vide Fara do mato.

Sabugueira do mato. Wilder Flieder. Aegiphila fuminensis

Tellos. Terbenaceate. Ber. d. Ph. G., 190う.

sabugueira da terra. Einheimischer Flieder. Vide Acapora.

Saca estrepe | Rolandra argentea Rotth. ComSaca estrepe de campina $\int$ positae.

Saca estrepe do mato. Microlicia Blanchetiana Cogn. Melastomaceae.

Sacarolha. Korkzieher. Ixora corrlifolia Wigh. Rubiacear. 


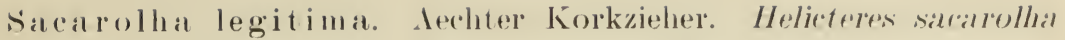

St. Hil. Sterculiareate. Ber. d. Plı. G., 1900, 1). ז́s.

Śacha-vay. Gustalia longiffora Popl). Lecythidaceate. Ler. d. l'h. (i., 1902, p. 229.

Sagâ, Sayaio. Kalanchoe lorasiliensis fanb. C'rassulaceale. Ber. d. Ph. G., 1901, 1). 20:3.

Saceubare. Cyrtopodinm sarnbare Barb. Roclerr. Orehidaceate.

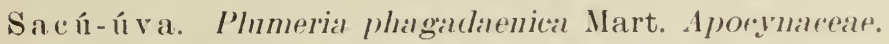

Salgueira. Weirle. Sallix martianal Leyb. Salicareale.

Salgueira falsa. Falsche Weide. Tonrnefortial Martii Fresc. Borlagillateale.

Salgueira de mato. Wilde W. Belangeral tomentosal Camb. C'u. noniaceae.

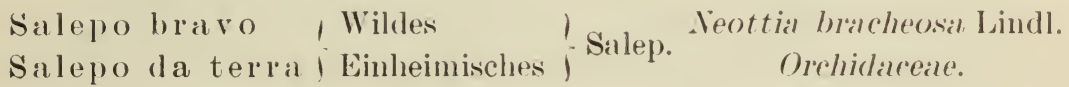
Sagueira. Sagopalme. Cycas revoluta L. C'rcadaceare.

Sagueira la terra. Einheimische Sagopalme. Ziamia Brogniartii

Wedd. Cycadareate.

Salsa brava. Wilde Salsa. Mikania apiitolia Dl. Compositale.

— (in Ceará) Ipomaea pés-caprase Sweet. Convolvulaceate.

_- (in Rio de Janeiro) Herrerial salsalparilhal Mart. Snilalceate.

- (in Rio (nrande do Inl.) Muehlenharhial sagittifolia Meisse. Polygonaceate.

Salsa de capim. Gras Salsa. Smilax campestris Grieseb. Snilaceae. Salsa do mato. Wildes. Rajania verticillata Vellos. Dioscorianceae. Salsa da praia. Küsten S. Ipomoeal acetosalofia Röm. et Sclıuck C'onvolvulaceae.

Salsaparilha. Smilax paprracea Poir. Snilaceate.

- (in Mina) S. officinalis Kth. Snilaceae.

_ (in Rio de Janeiro) S. procera M. Smilaceate.

- legitima. Aechte. S. syphilitica H. et B. Smilaceae.

- de mato. S. niticla, Grieseb. Smilaceate.

Stalva. Salbei.

| Salvia coccinea L. Labiatae.

Salva encarnado. Rothe Salbei.J Ber. d. Ph. G., 1904, p. 382. Salva de Pará. Cunila incana Bth. Labiatae. Ber. d. Ph. G., 1904 , p. 380 . 
Sialva de Pernambuco. Eupatorium asperulaceum Bth. C'ompositile.

Salva brava. Wilde S. Liplial gemiatil H. I3. Kth. Verbenaceate. Ber. d. I'h. (i., 1905).

Salva cheirosa. Wohlriechende s. Vide salva de Pari.

Salva do mato. Salvia macrocalyx Gard.) Labliatae.

Salva do sertao. Salvia altissima Pohl. Ber. (l. Ph. G., 19)4. Salva sylvestre vos. Salva do mato. I. 3\$1, 382.

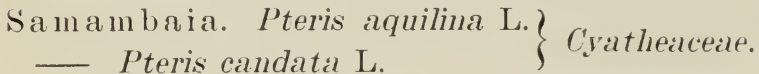

—_Mertensia dichotoma Sw. Gleicheniaceae.

S' a máma, Samauva, Samahuma, Sambauva, C'eiba Samauma

Schom. Bombaceate. Ber. 1. Ph. G., Berlin, 1900. p. 159.

Sambacaité. Vide Alfazema de caborlo. Ber. der Plı. G., Berlin, 1904 , p. 381.

śa mbucuim. C'ecropia syoka ph.vlla Mart. Urticaceae.

Sam ba iba. Ton Tupy-Caimba-rauh, iba-Baum oder Pflanze. Raublättrige Pflanze.

- C'ecropia scabra Mart. Lrticaceate.

- C'uratella Cambaiba St. Hil. Dilleniaceate.

$\left.\begin{array}{l}\text { Sambaibinlia } \\ \text { Sambauva }\end{array}\right\}$ curatella americana D. Dilleniaceate.

Sambaibinha do sertaõ. Davilla Martii Eichl. Dilleniaceate.

Sanamandé. Benennung fïr Sterva tostaõ im Staate Amazonas.

Śa nandí. Erythrina corallodendron L. Papilionaceae.

Sanandu do brejo. Sumpf s. Erythrina reticulata Presl. Papilionaceae.

San a nd ú-i. Erythrina falcata Bth. Papilionaceae.

Sangue de drago. Drachenblut. Croton echinocarpus Muell. Arg. Euphorbiaceae.

- Croton celtifolius Baill. Euphorbiaceae. Ber. d. Pl. G., Berliil, 190 ).

- Cioton hecatonandrus Muell. Irg. Énphorhiaceae. Ber. d. Ph. G., Berlin, 1905. 
— Croton salutiris Casarett. Euphorbiareae. Ber. d. Ph. G., Berlin, 190\%.

- C'roton Panlianus Muell. Arg. Enpliorbiareate. Ber, d. Ph. G., Berlin, 1905.

C'roton urncurana var. genuinus Muell. Arg. Euphorliaceae. Ber. d. Ph. G., Berlin, 1905.

Sangue de drago de folhalarga. Breitblättriges D. Croton peptocolyt Muell. Arg. Euphorbiaceae. Ber. d. Ph. G., Berlin, 1905.

Sangue de drago legitimo. Aechtes I). Croton erythraema Mart. Euphorbiaceae. Ber. 1. Ph. G., Berlin, 190天.

Sangue de drago da praia. Kïsten D. Croton migrans Casarett. Euphorbiaceae. Ber. d. Ph. G.. Berlin, 1905.

śangue de drage rastecro. Kriechendes I). Croton gracilipes Baill. var. genuinus Much. oby, Euphorbiaceae. Ber. d. Ph. G., Berlin, 1905.

Sangue de drago da Serra. Gebirgs I). Croton organensis Baill. Euphorbiaceae. Ber. d. Ph. G., Berlin, 1905.

- Croton oxyph.rllus Muell. Arg. Euphorbiaceae. Ber. d. Ph. G., Berlin, 1905.

Croton macrobothrys Baill. Euphorbiaceate. Ber. d. P'h. G., Berlin, 1905.

Sangue de Nosso Senhor. Christusblut. Molopanthera paniculata Turcz. Rubiaceae.

Sangue de Urubù. Aasgeierblut. Euphorbiaceae. Bauholz in Alagôas.

Santa Luzia. Vide Mata olhos. Euphorbiaceae.

Sapatas in ha. Kleiner Schuh. Miconia ciliata D. C. Melastomaceae. Sapateiro. Schusterbaum. Tibouchina grandifolia Cogn. Melastomaceae.

Sapé. Imperata brasiliensis Trin. Gramineae. Ph. Rdsch., New York, 1894 , p. 109.

Sapé macho. Männlicher S. Imperata vandata Trin. Gramineae.

Ph. Rdsch., New York, 1894, p. 109), 110. (Sapé corrumpirtes

Tupiwort von (apec-sengen.)

Sapiti und Pombeba. Bumelia obtusifolia R. et S. var. excelsa

Bg. Sapotaceae. Ber. d. Ph. G., Berlin, 1904, p. 32.

Sapoátas. Salacia attenuata Peyr. Hippocrateaceae. Ber. d. Ph. G., Berlin, 1902, p. 195. 
Sapotá

Sapotá grancle

I Grosse S. Achras sapota Mill. Sapotaceae. Ber.

Sapotî. Achras sapota Mill. var. sphaerica Bg. Sapotaceae. Ber. d. Ph. (i., Berlin, 1904, p. 41.

Sapoti-assú. Grosse s. Lucuma mammosa Gaertn. Sapotaceae Ph. Rdsch., New York, 1888, p. 6.

Sapoti dos Geringueiros. S. der Kautschuksammler. Vide

Guitiroba-assí. Ph. Rdsch., New York, 1888, p. 6.

Sapoti grande. Grosse S. Lucuma Bonplandia H. B. Rth. Sapo-

taceae. Ber. d. Ph. G., Berlin, 1904, p. 40.

Sapucaia, Sabucaia. Corrumpirtes Tupiwort von Sopia-Nest, Korb, aiaca - Eier-Nest mit Eiern. Lecythis Pisonis Camb. Le-

cythidaceae. Ber. d. Ph. G., Berlin, 1902, p. 228.

Sapucaia anaã. Zwerg Topfbaum. Lecythis nana Bg. Lecythi-

daceae. Ber. d. Ph. G., Berlin, 1902, p. 228.

Sapucaia assú. Grosser T. Lecythis amazonum Mart. Lecythi-

daceae. Ber. d. Ph. G., Berlin, 1902, p. 398. (Zwerg- und gross in Betreff der Frucht.)

Sapucaia branca. Weisser T. Lecythis lanceolata Poir. Lecythidaceae. Ber. d. Ph. G., Berlin, 1902, p. 229.

Sapucaia de castanlia. Kastanien 'T. Lecythis urnigera Mart.

Lecythidaceae. Ber. d. Ph. G., Berlin, 1902, p. 230. Arch. d.

Pharm., 1867, pp. 82, 42, 245, 253.

Sapucaia indôa. Mörser T. (Tupiwort). Lecythis grandifolia Bg.

Lecythidaceae. Ber. d. Ph. G., Berlin, 1902, p. 400.

sapucaïa inhahiba. Vide Sapoquema.

Sapucaià merim. Kleiner T. Lecythis Pohlii Bg. Lecythidaceae.

Ber. d. Ph. G., Berlin, p. 399.

- Lecythis angustifolia Endl.

Sapucaia pilaó. Mörser T. Vide Sapucaia glabra Camb. Lecy-

thidacec e. Ber. d. Ph. G., Berlin, 1902, p. 401.

Sapucaia-rana. Falscher T. Lecythopses.

Sapucaia-da varzea. Topfbaum der Ebene. Lecythis ovata

Camb. var. ovalifolia Bg. Lecythis bracteosa P.

sapoquema. Wohlriechender Topfbaum. Lecythis odora Popp.

Lecythidaceae. Ber. d. Ph. G., Berlin. 1902, p. 399. 
Sapucainha. Kleiner Topfbaum (Frucht). Carpotroche brasiliensis Endl. Flacourtiaceae. Ber. d. Ph. G., Berlin, 18!)9, p. 162.

Sapulá. Salavia laxiflora Peyr.,

- Salacia glomerata Peyr.,

p. 197 ; Hipporateaceas,

p. 195$\}$ Ber. 1. Ph. G.,

Saputi-assú. Grosses. Salacia paniculata, Peyr. I Berlin, 1902.

Saracura. Begonia hirtella L. Begoniaceae.

- Jussiaca nervosa Poir. Onagracene.

Sarapo. Nach Ule eine Hevea sp. Kautschukhaltige Milch liefernd. Sargasso. Sargassum bacciferum Ag. Fucaceae.

Sarnadinha. Leandra sp. Melastomaceae.

Saró. Trithrinax schizophylla Dr. Palmae. Ph. Rdsch., New York, 1889.

Sassafraz. Nectandra cymbarum Nees. | Lanraceae,

—_Acrodichirlium rhrysophylhum Meissn. (Ph. Rdsch., N. Y., '86.

Sassuaya. 'Tupiname fiir Herva de Collegio.

Saudades. (Gartenpflanze.) S'cabiosa atropnrpurea L. Dipsaceae. Saudades do brejo. Sumpfskabiose. Ternonia missionis Gardn.

Compositae.

Saudades de campina. Wiesen S. Asclepias nmbellata Vellos. Asclepiadaceae.

Scilla brasileira. Brasilianische) Meerzwiebel. Pancratium Guy. Scilla da terra. Einheimische $\}$ anenseke Amaryllidaceae.

Sebastiaí de Arruda. Physocalymma floribunda Pohl. Lythra-

reale.

Semperviva bravo. Wilder Inmergriin vide Pennaoho amarello Semperviva do mato. Waldimmergrin. Liangsdorfia hypogaea

Mart. Balanophoreae. Ph. Rdsch., New York, 1895, p. 34.

Senne do campo. Steppen Sennesblätter. Cassia cathartica Mart.

Caesalpinaceae.

Sensitiva. Simpflanze. Mimosa sensitiva L. Mimosaceae.

Sensitiva de folha grande. Grossblättrige S. Mimosa extensa

L. Mimosaceae.

Sensitiva legitima. Echte S. Mimosa puclica L. Mimosaceae.

Sensitiva do mato. Wald S. Mimosa vellosiana Mart. var. glabra Wawra. Minosaceae.

Sensitiva do mato. Gebirgs S. Mimosa tremula Bth. Mimosaceae.

Sendiba vide Mîlho cozido. 
Sepepira vide Angelim pedra. Zeitsch. d. Allg. Oest. Apoth. Ver., 1868, p. 517. Krrstall. Product.

Sepepira, Sebepira dos tentos. Armosia coccinea Jacq. Papilioñaceae. Samen Halsschmuck.

Seringueira amarellas. Gelber Kautschukbaum. Herea lutea

Ifuell. Arg. Euphorbiaceae. Ber. d. Ph. G., Berlin. 1905.

- Herea lutea Muell. Arg. var. 'uneaca Huber Euphorhiaceae. Ber. d. Ph. G.. Berlin, 1905.

Seringueira anans vide Anans.

seringueira barriguda. Bauchiger $\mathbf{K}$. Herea spruceana Muell.

Arg. und nach Cle noch eine andere Hevea sp. Euphorbiaceae.

Ber. d. Ph. G.. Berlin, 1905.

Seringuira blanca. Weisser K. Hereal brasiliensis Muell. Arg.

Euphorbiaceae. Ber. d. Ph. G., Berlin, 1905.

Seringueira mangue. Sumpf K. Hevea guranensis Aubl. Euphorbiaceae. Ber. d. Ph. G., Berlin, 1905.

Seringueira preta. Schwarzer K. vid. Seringueira branca, Euphorbiaceae. Ber. d. Ph. G., Berlin, 1905.

Seringueira puca und Puca seringa. Roter K. Hevea viridis

Huber Euphorbiaceae. Ber. d. Ph. G., Berlin, 1905.

Seringueira rana. Falscher K. rid. Sering. mangue. Euphorliareae. Ber. d. Ph. G., Berlin, 1905.

seringueira do Rio negro. Hevea discolor Mïll. Arg. Euphor-

biacear. Ber. d. Ph. G., Berlin, 1905.

Seringueira vermaldein. Echter K. vide sering. branca. Euphorbiaceae. Ber. d. Ph. G., Berlin, 1905.

Seringueira vermelha. Roter $K$., nach Ule eine Herea sp.

Euphorliaceae. Ber. d. Ph. G.. Berlin, 1905.

serpaõ do mato. Wilder Guendel. Gilechon marifolia Bth. La-

biatae. Ber. d. Ph. G., Berlin, 1904, p. 381.

Serpentaria brara. Wildes. Dorstenia tromiticum rotundifolia

Arrnd. Crticaceae. Ph. Rdsch., New York, 1891, p. 290.

Serpentaria de mato. Walds. Dorstenia Pernambucana Armd.

Urticarceae. Ph. Rdsch., New York, 1891, p. 290.

Serralho. Gemïsedistel. Sonchus oleraceus L. Compositae.

Sete casacas. Sieben Röcke (Bast sieben Lagen). Britoa Sellomiana Mrrtaceae. Ber. d. Ph. G., Berlin, 1903, p. 362.

Sete cascos. Bauholz in Alagoas. 
Sete folhas. Sieben Blätter. Diclvpanas longepetilatnm C. March. Hederaceate.

Sete sangrias. Sieben Aderlïsse. Begonia sanguinea Radd. Be goniaceate.

- Symplocos pubescens Kilotsch.

- Symplocos platyphylla Mart.

_ Symplocos parviflora Bth.

- Cuphea ingrata Cham. et Schlecht.

- Barberina tetrandral Mart.

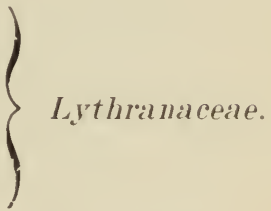

Shiringa-vana. Sipinm Marmieri Huber. Euphorlsiaceate.

sicopira, Sebepira, Sepipira, sicupira. Kautschuk liefernd.

Bowdichia virgiloides H. B. Kth. var. glilbrata Bth. Fand 1866;

in der Wurehrinde einen krystallinischen Bitterstoff. Sicopirin.

Nach Prof. I)r. Geuther in Jena $\mathbf{C}^{16} \mathrm{H}^{12} \mathrm{O}^{5}$ in der Zeitsch. d. Allg.

Oestr. Apoth. Verein. 1875, 1). 517. Petit publicirte seine Ent-

deckung dieser Substanz 1885 in Paris.

Sicopira ánacú. Bowdichial virgiloides H. B. Rth. Papilionaceae Sicopira áquoso. Wäissrige S. zufolge des reichhaltigen.Wassers in Stanme als Cerveja de sicopira. (s. Bier.) Vide Sicopira.

Sicopira assú

Sicopira branca

Sicopira guassú

Sicopira mijona

Vide Sicopira.

Sicopira mirim

Sicopira purda

Sicopira do campo. Acacia monacantha. Willd. Mimosaceae.

Sicopira u. Sepepira cary. Ormosia dasycarpa Jacq. var. minor Bth. Papilionaceae.

Sicopira falsa. Vide Sepepira amorella.

Sicopila felpuda. Haariger S. Pterodon pubescens Bth. Bowdishia virgilioides $\mathrm{H}$. B. Rth. var. pubescens Bth. Papilionaceae. Sicopira preta. Schwarze S. Bowdichia virgilioides H. B. Rth. var. ferruginea Bth. Papilionaceat.

Sicopira-una. Schwarze S. Caesalpinea ferrera Mart. var. Celostachya Bth. Caesalpinaceae.

Sicopira rermelha. Rote S. Bowdichia nitida Spruce. Papilonaceae. 
Silva. Brombeere. Rubus brasiliensis Mart. Rubiaceae.

Silvina. Polypodinm vaccinifolium Langsd. Cyatheaceae.

Simaruba. Vide Marupà.

Simira. Psychotria simira Röm. et Schl. Rubiaceae.

Simiri. Vide Jetahy.

Simúlo. Chrysophyllum sp. Sapotaceae.

Sipahúba. Combretum fractuosum Mart. Combretaceae.

Sipoátas. Vide Sapoàtas.

Sobrásil. Erythroxylon pulchrum St. Hil. Erythroxylaceae.

Soldanella d'agua. Wasser Drottelblume. Lymnanthemum Hum-

boldtianium Grieseb. Gentianaceae.

Solidonia. Vide Célidonia. In Amazonas ist Herva Tostaõ.

$\left.\left.\begin{array}{l}\text { Sorva } \\ \text { Sorveira }\end{array}\right\} \begin{array}{l}\text { Vogelbeere } \\ \text { Vogelbeerbaum }\end{array}\right\}$ Cónma utilis Muell. Arg. Apocynaceae.

Sorveira in Pará. Chrysophyllum excelsum Huber Sapotaceae.

Sorócó. Sóracca illicifolia Mig. Urticaceae.

suassú assú. Tupibenennung für Herva grossa.

Subragi. Frangula polymorpha Reis. Rhamnaceae.

Subrasil. Caesalpinia peltophoroides Bth. Caesalpiniaceae.

Sucuúba. Plumeria suonuba Spino.

- Plumeria fallax Muell. Arg. $\}$ Apocynaceae, auch Amapà.

Sucuúba rana. Falsche S. Pterandra pyroidea Tuss. Malpighiaceae.

Sumauma. Vide Samaúna.

Sumaré. Cypripedium colhurum Vellos. Orchidaceae.

Surúcúba da matta. Bauholz.

Surúcúra. Begonia macroptera Rltych. Begoniaceae.

Taa-assí. Vide Gito. Meliaceae. Ber. d. Ph. G., Berlin, 1901.

Taba-buya. Typha angustifolia Vellos. Typhaceae.

Tabaca. Vide Petum.

Tabaca-vana. Falscher Tabak. Pluchea quitoc D. C. Compositae. Tabaco. Vide Fumo.

Tabaco brava. Wilder Tabak. Vide Tumo bravo (Solanaceae).

Tabaco de Judeo. Judentabak. Vide Isca de mato.

Tabebuya. (Corrumpirtes Tupywort: ron Tacyba-Ameise, ibaBaum, bubuy-leicht. Leichtes Holz, in welchem Ameisen wohnen. Tabelenia longifolia Hook Bignoniaceae. Pantoffelholz der Brasilianer. 
Tabocea (in Tupy-Rohr). Guadua trinii) Arunchinaceae. Beyer.

in Ceará. Guadua paniculata Munro. $\int 1894.1 \% 112,169$.

Tabocca-ceem. ('Tupi - Sïsses Rohr.) Vide Tacomaie.

Taboquinha. Kleines Rohr. I'ommelinil agramia Kth. var. revens

Cenb. Commelinaceae. Ph. Rdsch., New York, 1892, p. 2.77.

Tabì. Tupiname fiir gebrannten '/ucker.

Tabuâ. Aeschynomene marginata Bth. I'apilionaceae. Śchlangenantidot.

Tabûa. Typha minor Willd. Typhaceae.

Tabía esbreita. Schmalblïtrige T. \} Typhaceae, eingefïhrt.

Tabí a larga. Breitblïtrige 'T'.

Tabúasinha. Vile Taboquinha.

Tacáca. Brei von Tapioca mit Tucupysauce.

$\left.\begin{array}{l}\text { Tacahan - aca } \\ \text { Tacamahaca }\end{array}\right\}$ Tupinamen fïr Alme-cegueira vermelha.

Tachi. Guitarrenholz. Tachigalia spec. Huber.

— Triplaris surinamensis Cham. var. Benthamiana Meissn. Polygonaceae.

- Sclerobium paniculatum Vog. Leguminosae.

'Tachi- niruñ. Kleines G. Swartzia acuminat: Willd. var. bridynamus Huber Leguminosae.

Tacoàra und Tagoàra. Tupiname für Taquara legitimo.

$\left.\begin{array}{l}\text { Tacoàra-cèem } \\ \text { Tacomarée }\end{array}\right\}$ Tupi - Siisses Rohr - Zuckerrohr.

Tacô̂ri. Benennung in Matto Grosse für Castanheiro de Maranhon.

Taconhoba. Bastfutteral der Indianer für Geschlechtsteile der

Männer.

Tacumbà - uva. Bactris inundata Mart. Palmae.

Ta deguy, Taguèlla. Conyza squarrosa L. Compositae, eingefiihrt.

$\left.\begin{array}{l}\text { Tagòag-i ba } \\ \text { Tagòa-iwa }\end{array}\right\}$ Tupiname für Tatagiba.

Tagóia - y. Vide Carurú amarello.

Tahamb̌̀, Tahüu - he. Vide Caa-ataya.

Tahúampá. Sapium biglandulosum var. 1 Miill. Arg. Euphorbiaceae.

Taia-boeira. Verkrippelte Mandiocapflanze.

Taijûba. Vide Tatagiba. 
Ta imağh, Taémagh. Irostigma atrox Miğ. Lrticaceae. Ph. Rdsch., New York, 1891, p. 167.

Taióba, Taipéba. Vide Tayôba.

Taioia. Vide Tayúra.

Tậa. Vide Taróba.

Tạá de cobras. Schlangen T. Dracontium asperum. C. Koch. Araceae.

Tajab-assu, Tajal, Tajaz. Vide Tayóba.

Tajará Tajùrá $\}$ Vide Tinchoraõ.

Tajassí-carapia. Tupiname für ('lara d'oros.

Tajassu-ubi. Hyospatha elegans Mart. Palmae. Ph. Rdsch., New York, 1889 , p. 262.

Ta macaré. (Tupi - Tama - weiblicher Geschlechtstheil, Koaré zusammenziehen.)

- Caraipa fascicuata Camb.

- C'araipa grandifolia Mart.

Ta ma coaré mirim. Kleine T. Caraipa glabrata Mart.

— Caraipa insidiosa Barb Rodr.

- Caraipa lacerdiosa Barb. Rodr.

Tamacoaré de igapo. Sumpé T. Caraipe palustris

Barb. Rodr.

Tamacoaré-rana. Falsche T. Caraipe spuria Barb.

Rodr.

Ta ma coaré - rêtè. C'araipa sylvatica Barb. Rodr.

Tamacoaré - landy, Tama onari. Balsam oder Oel der Caraipe.

$\left.\begin{array}{l}\text { Tametarana } \\ \text { Tamótarana }\end{array}\right\}$ Tupiname für Amendoion.

Tam anqueira. Pantoffelholzbaum. Goeppertia sericea Nees. rar. bracteolata Meissn. Lauraceae.

Tamanqueira a marella. Gelber P. Nectandra mollis Nees. rar. villosa Meissn. Lauraceae.

Tamaquaré. Malonetia tamaquarina A. D. C. Apocynaceae.

Tamará da terra. Einheimische Dattel. C'ocos Datil Gr. et Dr. Palmae. Ph. Rdsch., New York, 1888, p. 91.

Tamareira. Dattelpalme. Phoenix dactilifera L. Palmae. Kultirirt. 
Tam a rindeira. Tamarindus indicus L. Caesalpinaceat. Allgemein kultivirt.

Ta m bà-tajà. Xanthosoma atrovirens C. Koch. var. alpendiculata Engl. Araceae. Ph. Rdsch., New York, 1892.

Tambá - taríga. Xanthoxylum Riedelianum Lingl. Rutaceae. Ber.

d. Ph. G., Berlin, 1899, p. 345.

Tambor. Macrolobium hymenaeoides Willd. Leguminosae.

Tamboril. Enterolobium Timboura Mart. val. canescens Engl.

Miomsaceae.

Tambú. Rubachia laterifolia Bg.
Tambú a marello. Gelber T. Myrtaceate. Bauholz. Myrtaceae.

Tambú a meixa. Pflaumen T. Eugenial pruniformis

Camb.

Tambú rosa. Rosaroter T. Bauholz.

Tambureira. Atropischer Maiskolben.

Ber. d. Pl. G., Berlin.

1903, p. 22.

Tamburi. Vide Pindahyba.

Tamburil. Vide Timbauva.

Tambury. Vide Tamboril.

Tambuvi. Vide Timbóuva.

Tameorana. Dalechampia tryphilla Lam. Euphorbiaceae. Ber. d. Ph. G., Berlin, 1905.

Tamiarana, Tanjeraca. Tupiname für Urtiga cipu.

Tamujà, Tamuyá. Serjania tamuja Fr. Allem.

Ta mûjà. Vide Carurû cipó.

Tamûjo. Serjania glabrata Radlk.

Sapindaceae, Ber. d. Ph. G., Berlin, 1901, pp. $360,441$.

Tanasia. Rainfarn. Tanacetum vulgare L. Compositae. Gartenpflanze.

Tanohagem. Brasilianischer Wegerich. Plantago Guilleminiana

Desp. Plantaginaceae. Ber. d. Ph. G., Berlin, 1901, p. 209.

Tangará. Tupiname für Lanceta.

( Tangaraca-assu-vaa. Grossblättrige $\mathrm{T}$. Coccoloba crescen tiafolia.

Tangaracan. Cham. Polygonaceae. Ph. Rdsch., New York, 1895 , p. 35 . 
Tangeraca. Rubia noxia st. Hil. Rubiaceate.

Miconial guyanensis Cogn.

Vide Herva tostaõ. Ph. Rev., 1896, p. 82.

Vide Herva de rato.

Vide Laceta.

Tangerava assú. Grosse T. Ps rchotria, ruelliaefolia, Müll. Arg. Rubiaceae.

$\left.\begin{array}{r}\text { Tangerana. Triplaris surinamensis. Cham. } \\ \text { Triplaris Schomburgkiana Bth. }\end{array}\right\}$ Polygonaceae.

Tangerina

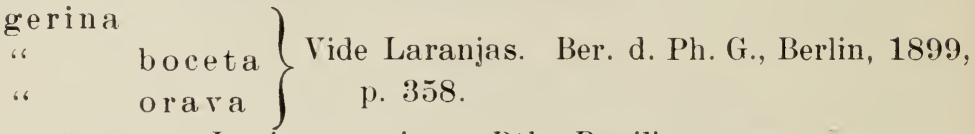

Tange-tange. Lupinus vaginans Bth. Papilionaceae.

Tanhoraó, vide Tinhoraó.

Tanibuca, Tanimbuca. Terminalia Tanimbouca

Smth.

Terminalia dichotoma Mog.

Terminalia crinita Bth.

Tapa-ceriba. Vide Battan.

Tapa - ceriba a marella. Gelbe T. Andradea floribunda Fr. Allen.

Nyctaginaceile. Ph. Rdsch, New York, and Ph. Review, 1896, p. $181-84$.

Tapa-ceriba branoa. Weisse T. Piconia alcalina Fr. Allen.

Tapal-cocca. Tupiname für Tentos (Ormosia).

Tapagiba. Vide Tatagiba.

Tapa-guela. Schlundstopfer. Cionidesia Casarettiana Bg. Myrtaceae. Ber. d. Ph. (i., Berlin, 1903, p. 22.

Tapa-ipi. Pancratinn gun̂nense Ker. Amaryllidaceae. Ph. Rdsch.,

New York, 189:3, p. 136.

Taperába, Taperóba. T'upirira guianensis Anb. var. elliptica

Engl. Allacardiaceae, Ber. d. Ph. G., Berlin, p. 155-157.

Taperába merim. Kleine T. Spondias macrocapa Engl. Anacardiaceae, Ber. d. Ph. G., Berlin, p. 155-157.

Taperiba. Spondias lutea L. Anacardiacea, Ber. d. Ph. G., Berlin, p. $155-157$.

Tapiß̀anga. Bignoniaceae. Heilmittel in Ceara.

Tapi - catì, Tapy-catí. Guarea tuberculata Vell. var. purgans C. Dl. Meliaceae. Ber. d. Ph. G. Berlin, 1901, p. 351.

Tapia, Trapia. Vide Gorarema. 
Tapichó. Siphonia rhytidocarpa Mart. Euphorbiareae. Auch Benennung iïr fossiles Kautschuk.

Tapiconha. Tupiname fïr Maismehl.

'Tapiconi, Tapicuy. Ein gegohrenes, bierähnliches Getränk der Indianer aus der Mandiocararibe.

Tapicur и́ fema. Weiblicher T. Bauhölzer in Sergope.

Tapicurí macho. Männlicher T. $\}$ Euphorbiaceae.

Tapin hoan. ('Tupi. pyhoan - Beıle, Auswïchse) Silvaea nal valuim Fr. All. Ph. Rev. der Lanraceae. Vorziigliches Sichifisbauholz. Wird vom Bohrwurm verschont.

Tapiuhoan de Olho de sapo. Bauholz in den Nordstaaten.

Tapioca. Tupiname des Stärkemehls der Mandioca.

$\left.\begin{array}{l}\text { Tapiqui-rana } \\ \text { Tapiranga }\end{array}\right\}$ Bignoniaceae. Heilmittel in Ceará und Amazonas. Tapirica. Bongainvillea glabra ('hois. Nyctaginacear. Ph. Rev., 1896, p. 54.

Tapixaba, corrumpirt von der 'Tupibenennung 'Tupixaba-Besen.

Taximgui. Vide Capixingui. Ber. d. Ph. G., Berlin, 1905.

Tapoca. Vide Taboca.

Tapororocca. Vide Caapororoca.

Tapúropá. Tupiname fïr Genipapo.

Tapurì. Euphorbiaceae. Kautschuk liefernd.

Tapyrá-coynana. Tupiname für Canna fistula do grande.

Tapyrá-pecú. Elephantopus hirtiflorus Dl. Compositae.

Tapyrá-peací. Tupiname für Lingua de vacca.

Taquàra. Rohr in Tupi. Nastus barbatus. Rup. p. 169.

Arundinaria amplissima Nees ab E., p. 169.

In Alagoas. Guadua exaltata Doell., p. 169.

In Amazonas. Guadua angustifolia Kth., p. 169.

In Cearà. Merostachis Riedeeiàna Rupr., p. 168.

In Pavà. Guadua merostachia Rupr., p. 169.

In Cambú. Bambus T. Chusqueil melythroides Ph. Rdsch., New York, Kth., p. 169.

In fina. Dïnne T. Vide Taquari.

Taquàra legitime. Echte T. Guadua Tagoara Kth., p. 168. 
Taquàra merim und T. minda. Kleine T. Merostachys Fischeriana Rup.

Arundinaria vertivillata Nees, p. 168.

Taquòra da serra. Gebirgs T. Merostachys ternata Nees, p. 168.

Taquàr-assú. Grosse T. Chusquea pinifolia Nees, p. 169.

Merostachys Clausseni Munro, p. 168. Merostachys Gaudichaudii Kth., p. 169.

Tuquàri. Merostachys Burchhellis Munro, p. 168.

Gramineae, Ph. Rdsch., New York, 1894.

In Alagoas. Panicum sanguinale L. var. longeglum Döll.

Taquari de Cavallo. Pferde T. Panacetum setosum Richard.

Taquari da matta. Urwald T. Vide Capim Cezerro.

Taquari de Goyana. Mabea taquari Anbl.

Taquari bravo. Wilde T. Mabaa taquari Ber. d. Ph. G., Aubl. var. genuine Müll. Arg.

Euphorbiaceae, Ber. d. Ph. G.
Berlin, 1905 .

Taquàrinha. Kleine T. Vide Taquari mirim, Chrisiúma und Uricaninha.

Taquarinha d'agua. Kleine Wasser-T. Commelina deficrens Hook Commelinaceae, Ph. Rdsch., New York, 1892, p. 258.

Taqueira. Vide Abobora d'agua. Ber. d. Ph. G., Berlin, 1900. Taragiba. Vide Tatagiba.

Taraira-moira. (Tupi - Taraira Fischart, moira - betäubend) Cocculus ineme Mart. Menispermaceae.

Taraira-vana. Falscher T- Serjanïa erecta Radlk. Sapindaceae. Ber. d. Ph. G., Berlin, 1901, p. 361.

Tarampabo. Oenocarpus tarampabo Mart. Palmae. Ph. Rdsch., New York, 1889.

Tarariaya, Tareriaya. Vide Bredo fedorento. Ber. d. Pl. G., Berlin, 1898, p. 43.

Tar a rûcú. Vide Pajòmariòba.

Tarenago. Cleome spinosa L. Capparidaceae.

Tarerique, Tarorequi. Tupyname für Mata pesto.

Taririque. Heilpflanze in Alagoas.

Taroman. Citharexylum cinereum L. Terbenaceae. Ber. d. Ph. G., Berlin, 1904.

In Pernambuco. Vide Maria Preta. 
Tarrago. Euphorbia lathyris L. Euphorbiaceae. Gartenpflanze.

'Tarúba. Gegohrenes Geträink aus der Mandiocariibe, bereitet von den Indianer'n.

Tasneira. Jacobs Kraut. Senecio Jacobaea L. ) Compositae. Von Tasneirinha. Kleines J. Senecio vulgaris L. S Portugal eingefülnt.

Tass úana. Tupiname für Anil assí.

T'atagiba, Tatayba. Gelbholz. Maclura affinis Miq. Tatagiba de campo. Steppen G. Machura brasiliensis Endl.

Tataiba, Tatai-y, Tataïba, Tä̈ba, Taragiba, Tatarémí. Nechtes Gelbholz. (Tupi., 1891 , Tata-i - feuerfarben, iba-Baum) Maclura tinctoria Engl.

Urticaceae, Ph. Rdsch. New York, p. 248, 290,
291.

Tatajúba de campo. Steppen T. Cassipourea spectabilis. Rhigophoraceae.

Tatapiriva. Protium giganteum Engl. Burseraceae.

Tatapiriva preta. Schwarze T. Heilpflanze in Alagoas.

Tatâ̂paca. Vide Mangue vermelho.

Tatayouba. Caryocar glabrum Pers. C'aryocaraceae.

Tateiba. Vide Tatagiba do campo.

Tatì. Gürtelthierbaum. Eugenia supraatilaris Sp. Myrtaceae. Ber. d. Pl. G., Berlin, 1903, p. 34.

Vazea indurata Fr. Allem. Olacaceae.

Tatú - catú. Vide Caa-ataya.

Tatúa-poca. Trichelia Barraensis C. Dl. ) Meliaceae. Ber. d. Pl. Tatúa- ïba. Trichelia Casaretti C. Dl. $\} \begin{array}{r}\text { G., Berlin, } 1901 \\ \text { p. 351, 352. }\end{array}$

Tatú-u. Vide Caa-ataya.

Tauary, Taurarè, Tauriri, Torari, Turari (Tupi. von turina femoralia) Couratari Taurari Bg. Myrtaceae. Ber. d. Ph. G., Berlin, 1902, p. 131, 402.

Taya-rana. Falsche Tagoba. Xanthosoma
violaceum Schott.

Ta y oba. Caladium poecile Schott.

Caladium esculentum Schott.

Araceae,
Ph. Rdsch., New
York, 1892. 
Tayoia. Trianosperma angustifolia Cogn., p. 319. Tayuya. (Tupi. - Frucht brennt wie Capsicum) Trianosperma Tayuya Mart., p. 319.

In Goyaz. Trianosperma tibirica Cogn., p. 324. In Para. Vide Tayoia.

In Rio Grande do Sul. Trianosperma ficifolia Cogn., p. 320.

Willbrandia villosa Cogn., p. 312.

Tayuya branca. Weisse T. Cayaponia hirsita Cognn., p. 318.

Cucurbitacer. Ber. d. Ph. G., Berlin, 1900.

Tayuy a grande. Grosse T. Cayaponia fluminensis Cogn., p. 318.

Tayuya guiabo. Willbrandia hibiscoides Manso, p. 312.

Tayuya mari. Vide Abobrinha do mato, p. 324. Tayuya pimenta comari. Comarimpfeffer $\mathrm{T}$.

Trianosperma Martiana Cogn., p. 324.

Tayuya das restingas. Küsten T. Vide Batata de purga.

Teajú. Tragia volubilis L. var. genuina Muell. Arg. Euphorbiaceae.

Ber. d. Ph. G., Berlin, 1905.

Vide auch Cipo vermelho.

Teipóca. Aspidosperma bicolor Mart. Apocynaceae. Bauholz.

Tеiи́, Tejú. Grosser Eidechsenbaum. (Tupibenennung für grosse

Eidechse) vide Medicineira. Vide auch Herva de purgante. Euphorbiaceae. Ber. d. Ph. G., Berlin, 1905.

Teiú-assú. Vide Caapia assín.

Teiú cyca. Tupiname für Harz von Casearia resinifera Spruc.

Flacourtiaceae. Ber. d. Ph. G., Berlin, 1899, p. 169.

Tejuiba. Adenoropinm opiferum Mart. Euphorbiaceae.

Teiú - iba. Vide Paò de lagarto.

Tematarà, Tematarú. Xanthoxylum Chiloperone Engl.

Teita - capéba. Tibouchina gravilis Coq. Melastomaceae.

Teixò, Taxus baccata L. Taxaceae. Kultivirt.

Tembé - tariba, Tembai - tariba. Tupi. tembé - Unterlippe, tari-kleiner Pflock, iba-Baum, Lippenpflockbaum. Xanthoxilum rhoifolium Lam. Rutaceae, Ber. d. Ph. G., Berlin, 1899, p. 344,345 . 
Tembe - tarii (tarii - mittelgross). Kanthoxylnm Longsdorffii Mart. Ruticeae, Ber. d. Ph. G., Berlin, 1899, p. 344, 34.).

Tembe - tarnga, 'Tembe-tarí. (Grosser Pflock.) Tanthoxylnm rhoifolium var. pubescens St. Hil. Rutaceate, Ber. d. Ph. G., Berlin, 1899, p. 344,345 .

Xanthoxy/nm elegans Engl.

Tembiú - catí. 'Tupiname für Mangabeira. 'Tembiń - Nahrung, catí - schmacklıafte-

Tempéro do diabo. Teufelsgewiirz. Abobra tenuitolia Cogn.

Cucurbitaceae, Ber. d. Ph. G., Berlin, 1904, p. 315.

Tendy - iba. (Tupi. tendy-Speichel, iba-Pflanze), Pflanze, welche

speichelfluss verursacht. Spilanthes brasiliensis Spreng. Comjosit:e.

Tentos. Hals- und Armbandperlen. Ormosia nitida Vog. Tentos coral. Korallenperlen. Alenanthera pavoninaL. Tentos de enfeitos. Schmuckperlen. Vide Olho de plato.

Tentos graudes. Grosse Schmuckperlen. Ormosia Papilionae, dasycarpa Turks. ceae.

Tentos miudos. Kleine Schmuckperlen. Rhynchosia minima Dl.

Rhynchosia phaseoloides I)]. Vide Jequirity.

Tentos pretos. Schwarze Perlen. Connarus Patrisii Planch. Connaraceae.

Tentos vermelhos. Rote P. Vide Olho de pato.

Teriry, Tiriry. Myrtus umbilicatus Camb. Mvrtaceae. Ber. d. Ph. G., Berlin, 1903, p. 35.5.

Testa de boi. Ochsenstirn. Echyrospermum Balthazorii Fr. Allem.

Chrysobalanaceae. Sehr festes Möbelholz.

Testa de burro. Eselstirn. Bauholz. Leguminosae.

Tetim. Strutanthus vulgaris Mart. | Loranthaceae, Tety-poteira, Tety-potéiba. Vide Guira $\}$ Ph. Rdsch., New repoty. York, 1891.

Thiino. Thuja orientalis L. Coniferae. Von Portugal eingefuihrt. Thio-thió. Tupiname für Dendéoel, vide Coqueira Dendé. Ph. Rdsch., New York, 1888.

Thôa. Tupi - Blutfrucht. Gnetum Leypoldi Tul.

In Parà. Gnetum Thoa, Anbl.

Gnetaceae, Ph. Rdsch., N. York, 1893, p. 136. 
Thora. Ranunculus Thora L. Ranunculaceae. Von Portugal eingefïhrt.

Ti. In Tupisprache heisst-Saft. Corylene terminalis Endl. Liliaceae. Tiassè. Attalea Humboltiana Spruc. Palmae. Ph. Rdsch., New York, 1889, p. 133.

Tibàca. 'Tupibenennung, der Spatha der Palmenblüten.

Tibori. Vide Tembiú-catú.

Tiborna. Plumeria drastica Mart. Apocynaceae.

Ticorá, Ticorió. Galipea jasminiflora var. tenniflora Eng. Rutaceae. Ber. d. Ph. G., Berlin, 1899.

Ti i ùn a. Pfeilgift. Oide Urari.

Tijéla. Feingepulverter Schnupitaback.

Tikira. Tupi-betäubender Saft, vide Lirio amarello de campo.

'Tilho. Tilia tomentosa Mönch. Tiliaceae. Portugiesische Droge.

Timbakyba rana. Falsche T. Mimosa laxa Vallos. Mimosaceae.

Tim ba ù ba. Leguminosae. Bauholz.

Timbó. Tupi. Fischgiftpflanze. Serjania lethalis

St. Hil.

Vide Cipó Cimbó.

Timbó - aitica. Vide Timbui cipo.

Timbó-amarella. Gelbe T. Serjania ovalifolia

Radlk.

Timbó-assí. Grosse T. Serjania Lourettiana

\section{Camb.}

Timbó boi. Ochsengift. Cleobulia multiflora Bth. Papilionaceae. Timbó Boticario. Officinelle T. Lonchocarpus Peckoltii Wawra.

Papilionaceae. Zeitschr. d. Allg. Oestr. Ap. Ver., 1881, p. 193 bis 208. Flora Regensburg, 1864, No. 15, p. 230.

Timbó branco. Weisse T. Serjania glutinosa Radlk.

Timbó braco. Wilde T. Serjania erecta Radlk. Timbó cabelluda. Behaarter T. Serjania cuspidata Camb.

Timbó cipo. Sianen T. Vide Timbó titica. Timbó de folha grande. Grossblättrige T. Vide Tingui de cesto.

Ti mbó go ra nà. Tupi-GiftgetränkT. Vide Timbó raiz.

Timbó grande. Vide Timbó assú.

Sapindaceae, Ber. d. Ph. G., Berlin, 1901, $358-360$. 
Timbo guassis. Sehr grosse T. Derris nigrescens Bth. Papilionacear. Oenocarpus firox Mart. I'alnale. Kufolge der bösartig verwundenden Stachelı. P'r. Rdsch., New York, 1s89).

T'imbó legitimo. Wehte T. Serjania acmminatia Radlk., p. :361.

T’imbó de leite. Milchige T. Srryanial noxial Camb., p. 361 .

Timbó de mato virgem. Irwald 'T. I'anllineal spicatil Bth., p. $4+1$.

Timbó mirim, T. mindo. Kleme T. Serganial communis Camb., p. 3666.

Timbó-pêba. Grossbliattrige T. Panllineal molialefolial T'uss.

Magonial glalnatal St: Hil., 1. 112.

Timbó peise. Fisch T. Serjantia irhthyortonal Rallk., p. 364.

Serjania cuspidiltal st. Hil.

Vide Timbó cabelludo.

Silpindinceate. Ber. 1. Ph. (i., Berlin, 1901.

Timbó piranga. Rote T. Vide Timbó verdaleiro.

Timbor raiz. Wurzel T. Comptosemar cortalcenm Bth. Papilionaceae. Timbó rana Falscher T. Bauholz in Amazonas.

Timbó das restingas. Riff T. Serjanial rentata Radk. Sapindirceate.

Timbó do Rio de Janeiro. Phrsalis heterophylla Nees ab E. Solanilerile.

Timbó do Rio Grande. Panullinea australiss St. Hil. Śapindaceae, Timbó de sianta ('atharina. I'anllineal carpo- Ber.d.Ph.G., podeas, C'omb. Berlin, 1901, p. 442

Timbó le S. P’aulo. P'sychotria Marrgralrii spieng. Rulpiaceale. Timbó-titica. Stinkende T. Serjanial grandiflora, Camb., p. :360.

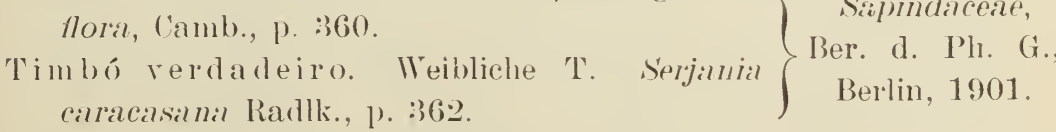

Timbó- ïva t Timbóbaum. Vide auch Timbouva.

Timbónva. Timbohaum. Enterolobinn Timbonva Mart. Mimosaceile.

T’i nóba. Vide Tabebuya.

Timbori, Timburi, Timbór. Vide Tambór. 
Timontou. Polygala Timonton Anbl. Polygaleae.

Timpa-pêba, Timbubeba. Grosses Fischgiftblatt. Ruprechtia laurifolia Miq. Polygonaceae. Ph. Rdsch., New York, 1895, p. 36. Tinamalú. Vide Mûhi.

Tinjé. Vide Timbo pêba.

Tingé-chia ) Tupiname für Marmelinho (Tournefortia.) Ber. d. Tingé-cuiat Ph. G., Berlin, 1902, p. 1:38.

Tingù a ciba. Der richtige Name ist: 'Tingoa-assú-üba. Tingaweiss, assú-gross, ïba-Baum. Grosser weisser Baum. Rutaceae. Xanthoxylum tinguaciba St. Hil. Ber. d. Ph. G., Berlin, 18.99, p. 347 .

Ting ùa - üba. Vide Tupúróba.

Tingu i (nach Tupi-starkes Fischgift) Serjania clematiclifolia Camb.

Sapindaceae. Ber. d. Ph. G., Berlin, 1901, p. 361.

Tingui. Vide Tamìna.

Lupinus crotala rioides. Mart. Papilionaceae.

Tingui-ataitica. Vide Tingui cipó.

Tingui de Amazonas. Panllinea capreolata Radlk., p. 441.

Tingui capêta. Teufelsgift. Vide Timpa-pêba

Magonial pubescens St. Hil., p. 112.

Tingui de Cayenna. Vide Anil bravo.

Tingui de cesto. Korb T. Serjania pauciden-

tata D. C., zum Flechten, p. 321.

Tingui cipò. Lianen T. Paullinea arigona Vell., p. 444.

Tingui-cola. (Tupi-Schwanz T., wegen der Blüte.) Vide Timpa-pếba.

T'ingui de follua grande. Grossblätterige T.

Paullinea elegans St. Hil.

Tingui de frechas. Pieil T. Euphorlbia cotonoides Miq. Euphorbiaceae.

Tingui de gentio. Indianer T. Solanum toxicarium Lam. Solanaceae. 
Tingui de peixe. Tisch T. Serjania piscatormm Radlk. Sapindilceae. Ber. d. Plı. G., Berlin, 1901, p. 262.

Nissolia fiuticosa Jacq. Papilionaceae.

Phyleanthus piscatorum Kth. Euphorbiaceae. Ber. d. Ph. G., Berlin, 1905.

Tingui piranha. Piranhafischgift. Vide Cipo de macaco.

Tingui da praia. Küsten T. Jacquinia armillaris Jacq. Myrsinaceae. Tingui timbo. Vide Anyl bravo.

Tingu i vermelho. Rote T. Panllinea sericeas st. Hil. Sappindalceale. Tinhoraó. Caladium bicolor Tent. I Araceale, Ph. Rdsch., Yew Calialium picturatum C. Koch. i York, 1892, p. 28:3.

Tinilha. C'lethra tinifolial sw. Clethraceap. Von Portugal eingefiihrt Tinta de sapateiro. Schusterfarbe. Mironial albicans.

Mironial ceramicarplal ('oyn.

Melastomaceare.

Tintureia. Färbepflanze. Vidle Reseda amarella.

Tintureira vulgar. Gemeine Färbepflanze. Vide Carurú-assí. Are'.. d. Pn., 1856, p. 229. Ph. Ridsch., New York, 189.5, p. 269.

Tinteiró. Bauholz in Alagôas. Coccoloba excelsal Bth. Polygonareile. Ph. Rdsch., New York, 1895, p. 37.

Tipi. Vide Pipi.

In Maranhaó vide IIncarí-cai.

Tipi bravo. Wilde T. Mohlana nemolalis Mart. I'hytolaccarceate. Ph. Rdsch., New York, 1895, p. 216.

Tipi manso. Milder T. Pavonia paniculata Cav. Milvaceae. Ber. der Ph. G., Berlin, 1900, p. 270.

Tipiaca, Tipiacica. Tupibenennung für 'Tapiocaqualität.

Tip ù. Tipuana speciosa Bth. Leguminosae.

'Tiquira. Gegohrenes Getränk der Indianer aus Mandiocakuchen. Ph. Rdsch., N. Y., 1886.

Tiriba. Callisthene mollissima Warm. var. mucronata Engl. Vochysiaceae. Gerbematerial.

Tiririca, Titirica. Seleria brasiliensis Kth. Tiririca grande. Scleria cyperina Kth.

Tirirical de mato. Mastigoscleria reflexa N.abE. $\begin{gathered}\text { York. } \\ \text { Tirité }\end{gathered}$ Tirité. Calathea zebrina Lindl. Marantaceae. Verwildert. Ph. Rdsch., New York, 1894, p. 8t. 
Tissará. Vide Assay, Assahyl und Caquiero assahy. Ph. Rdsch., New York, 1889, p. 217.

Titará. Vide Espinha de diabo. Ph. Rdsch., New York, 1888, p. 26.5 .

Tithimalo. Euphorbia canescens L. Tithimalo bastardo. Euphorbia sylvatica Jacq.

Tithimalo do breja. Euphorbia palustris E. $\quad$ Drogen.

Toà, Túa. Vide Thòa.

Tocajé. Rhopala tomentosa Pohl. Proteaceae.

Tagùssú-carapia. Cordia intermedia Fresc. Boräginaceate. Ber. d. Ph. G., Berlin, 1902, p. 230.

Tojó mollar. Stechginster. Genista lusitanica L. I Drogen Tojó. Ulme. Llex europaeus L. $\quad \int$ von Portugal. Tolanga. Stachys arvensis L Labiatae. Ber. d. Ph. G., Berlin, 1904, p. 385.

Toma roda. Cyphomandra calycina sendt. Solanaceae.

Tomate. Liebesapfel. Lycopersicum esculentum Mill. Solanaceae. Arch. d. Pharm., 1857, p. 110.

Tomate cabeçuda. Kopf-Liebesapfel. Lycopersicum esculentum var. pyriforme Mill.

Tomate grande. Grosser L. Lycopersicum esculentum L. var. maximum. Solanaceae.

Tomba. Vide Espelina. Ber. d. Ph. (4., Berlin, 1904.

In Minas vide Batata de purga.

Tomilho, 'Thyman. Thymus vulgaris L. Labiatae. Kultivirt. Tomilho sylvestre. Wilder T. Lippia geniculata H. B. Ktl.

Verbenaceae. Ber. d. Ph. G., Berlin, 1904, p. 470.

T'on hôa - ra m beovia. Tupiname für Verbasco.

Topinam bor. Helianthus tuberosus L. Compositae. Kultivirt. Topa. Ochroma lagopus Sw. Bombaceae.

Toquillo. Vide Jijijapa.

Tortulho. Vide L'rupé.

Tussilagem. Huflattig. Tussilago farfara L. Compositae. (off. Droge.

Totai. Acrocomia Totai Mart. Palmae. Rd. Rdsch., New York, Totanga. Tupiname vide Cordaó de frade. Ber. d. Ph. G., Berlin, 1904 . 
Touca de viuva. Wittwenhaube. Petraea insignis Schum. Ter-

benaceae. Ber. 1. Ph. G., Berlin, 1904, p. 478.

'Touri. Vide Humery.

Tozarí. Vide Tissalı́.

Trabúco. Śchale der Śapueaiafrucht.

'Tracúa. Śchildkrötenfrucht. Monstera deliciosa Liebm. Araceae.

Tracì a m. Vide Jumbí.

Trana - bôta. Siderodenclron floribundum Vahl.

Siderodendron inflorum Vahl.

Míolopantinera paniculata 'Turez.

Rubiaceate.

Trapia. Capparis cynophallophora L. val. normalis Engl. Capparidiceae. Ber. 1. P'h. G., Berlin, 1898, p. 46.

Trapia (lo mato. Wilde T. Vide Cáapia und ('ontraherva. Ph.

Ralsch., New York, 18!)1, p. 291. Vide Ligna-osso.

Trapó. Lappenbaum. Maytenus racemosa Reiss. C'rastrinoeae.

Trapoeraba, Trapoerava, Trapuerara,

Trapeorava. Corrumpirtes Tupiwort. Trá descantia dinretica Mart.

Trapoeraba - aussì. Grosse 'T'. Commelina robustia Kth., 1). 257.

Trapoeraba - merim. Kleine T. Vide Trapoeraba vulgas.

Trapoeraba-vana. Falsche 'T. Commelina deficiens Hook, p. 258.

Cimmpelia Zanoni Richt., p. 257, u. Arelı. d. Pharm., 1860, p. 167.

('ommelinaceae, Ph. Rusch., New York, 1892.

Trapoeraba-tinga. Weisse 'T. C'ommelina Scabrata Seub.

Trapoeraba piringa. Rote T. Vicle Trapoerava vermelha.

Trapoeraba vulgar. Gemeine 'T. Commelina agraria Kth., p. 240, 257.

Trapo m a nga. Vide Trapoerava-vana (Campelia)

Tremate. Vernonia scabra Pers. Compositae.

Trenoços. Wolfsbohne. Lupinus albus L. Leguminosae. Kultivirt. 
Trepoerava. Commelina Pohleana Seub. p. 258. In Rio Grande do Sul. Commelina elegans, Humb., p. 257.

Commelinaceae,

Trepoerava ephemera. Kurz dauernde T. Ph. Rdsch.,

Commelina geniculata Vell.

New York. 1892.

Trepoerava vermelha. RoteT. Dichorisandra

penduliformis Kth.

Tres irmãos. Irei Briider. Smidelia salpicarpa Radlk. Sapindaceae. Tres folhas. Dreiblitt. Helictes multiflora Engl. Rutaceae. Ber.

d. Ph. G., Berlin, 1899, p. 3.54.

Allophylus sericeus Radlk. Sapindaceae. Ber. d. Ph. G., Berlin, 1902 , p. 103.

Tres folhas brancas. Weisses Inreiblatt. Vide

Acapú rana.

Ruticeae,

Tres folhas do mato. Wildes I)reiblatt. ('hu- Ber. d. Ph. G., paferro.

Berlin, 1899,

Tres folhas vermelhas. Rotes Dreiblatt.

Esenbeckia febrifuga Juss.

p. 336 .

Tres Marias. Drei Marien. Bougainvillea pomacea Chois. Nyctaginaceae. Ph. Rdsch., New York, 1896, p. 51.

Trevo. Klee. Trifolium polymorphum Poir Leguminosae. In Rio Grande verwildert.

Trevo d'agua. Wasserklee. Oxallis sepium St.

Hil. Oxaliclitepile.

Trevo amargosa. Blätterklee. Oxalis amalal l'h. Review,

St. Hil.

Madison,

Trevo azedo. Sanerklee. Oxalis barrelieri Jacq. 1899, p. 46-48.

Trevo azedo do campo. Steppen - Sauerklee.

Vide Trevo de Campo.

Trevo do brejo. Sumpfklee. Cleome paludosa Willd. Capparidaceae. Trevo de campo. Steppenklee. Oxalis cordata St. Hil. Oxali daceae.

Trevo carvalho, Honigklee. Melilotus officinalis Willd. PapilioTrevo cheiroso $\int$ naceae. In Rio Grande kultivirt.

Trevo de If to. Waldklee. Psychotria rubra Muell. Arg. Rubiaceae. Trevo rasteiro. Kriechender Klee. Oxalis corniculata L. Oxalidaceae.

Triaga. Theriakpflanze. Aristolochia theriaca Mart. Aristolochiaceae. Ph. Rdsch., N. Y., 1893, p. 182. 
Trifolio hat dieselben Pflanzen wie alle 'Trevoarten mit gleicher' Benemung, nur

Trifolio grande. (irosses Dreiblatt. Oxallis rioliarea Vellos. Oxalidiceale.

Trigo. Weizen. Tritirum vulgare Vill. Grannineae. Nur in Rio Grande kultivirt.

Tripa de galinha. Hïhnerdarm. I'ignal luteolal Ith. Dillbergia grantis Bth. $\}$ Palpilionaceate.

Trombeteira. Trompetenblume. Tatura arboreal L.) Solanalcale,

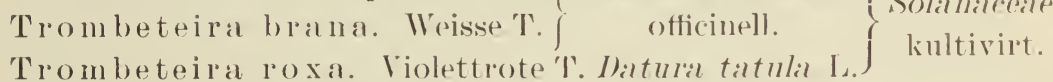
Troviseo, Trovisqueira. Dilphne gnidinm L. Daplmoidareae.

In Giiirten.

Trúcúas. Vicle Cipó lumbé.

Tuai-assí. ) Grosse T. Vide Marinheiro de folha larga. 'TupiTua- ïra. $\{$ benennungen.

Túapóca. Vide Teipóca. ) Grosse T. Guarea cermua Vellos. JeliaTúán-assu, Túaússú. J Grosse T. Giuareal cermual Vellos. Jelia-

Túca, Tóka. Vide Castaulıa de Párí. T'upiname.

Túcaia. Rhopala macrophylla schott. I'roteaceate.

Túcar i, Tócarór. Tupiname für. Castanla de Maranláo.

Tuсum, T'iсuи, Tocum. Tupibenenung für Bast, Faser.

_ Bactris maraja Mart. var. Limnala Trl., p. 20:3.

- Bactris acanthocarpa Mart., p. 203.

- Astrocaryum sclerophyllum Dr., 1) 263.

— in Matto Grosso. Bictris cuyabensis Barb. Rodr.

— in Pará. Vide Tucuman.

— in Rio de Janeiro. Vide Tucum de breja.

Tucum-assí. Grosser T. Astrocarjum rhonta Mart., p. 263. Astrocaryum leiospatha Barb. Rodr.

Tucum-assú da Serra. Grosser Gebirgs T. Astrocaryum leiospatha var. Sabulosum Barb. Rodr.

Tucum bravo. Wilder T. Astrocaryum volgare Mart., p. 264.

Tucum do brejo. Sumpf T. Bactris setosil Mart., p. 203. 'Tucum caivé (von Tupi : cai-sauer, vé-essen). Saure Speise.

Bactris inundata Mart., p. 204. 
Tueum do campo. Steppen T'. Astrorarymm echinatum Barb. Rodr.

Tueum de fruito ajedo. T. mit saurer Frucht. Bactris glancescens Dr., p. 203.

Tucum de fruito doce. 'T. mit süsser Frucht. Bactris piscatorum Wedd., p. 20:3.

'Tucum liso. Glatter' T. Astrocarym arenarium Barb. Rodr.

Tueum-marreya. Bactris Mindellii Barb. Rodr.

Tueum mirim. Kleiner T. Bactris Matto Cirossensis Barb. Rodr.

Tucum mirim do mato. Kleiner Wald T. Bactris Chapadensis Barb. Rorlr.

Tucum rasteiro. Kriechender T. Astrocarrom Weddellii Dr. p. 264.

Tucum rasteiro do breja. Kriechender Sumpf T. IDesmoncus setosus Mart., p. 266.

Tucumã, Tucuman. Astrocaryum segregatum I)r., p. 264. Tucumã, Turuman assí. Grosser 'T. Astrocarylum princeps Barb. Rotr., p. 266.

Tucumã legitimo. Echter T. Lstrocaryum Tucumal Mart. 1). 264 .

Tucumã rana. Falscher T. Vide Tucum do breẹo.

Tucuma da serra. Gebirgs T. Astrocaryum tucumoides Dr., p. 264.

Tucuma da terra firme. T. des Festlandes. Lstrocaryum candescens Barb. Rodr., p. 265.

Tucum̃- i. Gegolırenes Getränk der Indianer aus dem Safte der 'Tucumã legitima.

Tucuma-y. Vide 'Tucumã legitima.

Tucupi I Eine von Indianern allgemein benutzte Sance aus Tucupin dem eingedickten, mit Capsicum gewïrten Safte der Wurzelrübe von Maniliot utilissima Pohl.

Tueupi-pixuna. Sharare T., wenn die Since zur Extractconsistenz abgedampit.

Tucupi-ica. Wenn die Tucupi sauce mit Mandiocamehl und Waldgewïrzen als Masse bereitet. Zeitsclı. d. Allg. Oestr. Ap. Yer. 1881, p. 514. Ph. Rdsch., New York, 1886, p. 15т.

T'úgu i - ïba. Tupiname fïr Sangue de drago. 
'Tuhira. Vide Tukira.

Túià. Smilax oblongifolia Pohl. Ph. Rdsch., New York. S'milaceae. 'Túì̀-membêca. Heilpflanze in Pará.

Tuijuva. Vide Tatagiba.

Tuinan- iiba, Tuinan-túba, Tuinam - túba. Tupiname für Samandú.

Tukira, Tuquira, Tukayra. Amiryllis princeps Soln Dyk. Amaryllidaceae. Pharm. Rdsch., New York, 1893, p. 1:35.

Tumbaca, Tumbansa. Indianische Speisen von geröstetem Capsicum.

Túna. Opuntial tunil Mill. C'actaceate. Kultivirt.

Tuperaba. Vide Taperaba.

Tuperaba in S. Paulo und Matto Grosso. Taperiral Marchandii

Engl. Tapeira guianensis Anbl. var. cuneata Engl. Anacardiaceae, Ber. d. Ph. G., Berlin, 1898, p. 157.

Tuperiba, Tuperoba. Tapeira guianensis Anbl. var. elliptica

Engl. Anacardiaceale, Ber. d. Ph. G., Berlin, 1898, p. 157.

'Tupicha, Tupitcha. Sida acnta Burm. var. carponifolia $K$.

Schun. Malvaceae.

Tupixaba, Tupixara. Tupi-Besenkraut. Vide Vassourinha (Sooparia).

Tupoipi. Vide Urícatù.

Tupurapo, Tupúrôba. Tupiname für Caferana. Ber. d. Plı. G., Berlin, 1899, p. 226.

Tupi - cúri. Indianische Benennung für Pinheiro do Brasil.

Tuquira a marella. Gelbe T. Amaryllis Belladonna Vell. Amarylliclaceae.

Turanja. Citrus ponum Adami Rissa. ) Rutaceae, Ber. d. Plı. G., Turanja azeda. Citrus decumama L. $\}$ Berlin, 1899, p. 360.

T'úriùva. Licania turinva Cham. et Schlecht.

Moquilea turiuva Cham.

Hirtella octandra Willd.

In Amazonas. Licania macroplyylla Bth.

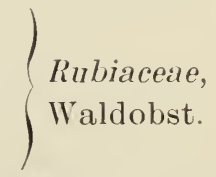

Turiunì-rana. Falsche 'T. Cordia cuyabensis Mans. et L'Holep.

Borayinaceae, Ber. d. Ph. G., Berlin, 1902, p. 131.

Turary. Paullinea grandiflora St. Hil. Sapindaceae. Ber. d. Plı. G., Berlin, 1901, p. 441.

Túrurú. Sterculia Ivira Anbl. Sterculiaceae.

Turúry. Vide Bussú. Pharm. Ridsch., New York, 1889, p. 262. 
Turs. Vide Goajerú.

Tury - atíapoca - assú. Tupiname für Marinheiro de folha larga. Tut ú. Brasilianische Nationalspeise. Masse ron schwarzen Bohnen, Mandiocamehl und gebratenem Speck.

Tut í-auba. Trichelia C'alsaretti C. Dl. Meliaceae. Ber. d. Ph. G., Berlin, 1901, p. 352.

Tutú-rúbà Lncuma chrịsophrolloides A. Dl. Sapotaceae. Ber. d. Ph. G., Berlin, 1904. p. 40.

Tycupy. Tupibenennung des Saftes der Wurzelrübe ron Manihot utilissima Pohl.

Tykyra. Virle Tukira.

Typiraty. Indianische Benennung für Mehl aus nicht ausgepressten und getrockneten Scheiben der Mandiocariibe.

Typyty. Bastsack der Indianer zum Auspressen der geriebenen Wurzelrübe von Manihot utilissima Pohl.

Ubàtimo. Vide Barbatimaio.

Lacá. Lucuma marginata Mart. et Eichl. Sapotaceae. Ph. Rdsch., New York, 1888.

Uacá-purana. Schwarze L. Luchma ramiftora A. Dl. Ph. Rdsch., New York, 1888.

Lacá-rańa-repoti. Lolanthaceae.

Lacari - côará. Bauholz am Rio branco (Amazonas).

Uacáuan. Vide Olho de boi.

Laçai. Vide Assalı̣.

Uacumân. Vide Coqneiro do campo. Ph. Rdsch., New York, 1888, p. 90.

Uacym-dyba. Tupy: ua - Stengel, cym - glatt, yba - baum.

Krilia brasiliense Barb. Rodr. Malraceae. Ber. d. Pl. Ges., Berlin, 1900, p. 268.

Uaimi. Astrocalyum Sthaimi Mart var. Orbignii Dr. Palmae. Pl.

Rdsch., New York, 1888, p. 264.

Ua ju rí. Vide Gonjerù.

Ua nà. Vide Arriozes.

Uan acú, Unacú. Tupybenennung der Samen ron Bixa orellana L. Uanixi, Uhanixi. Tupiname für Tentos grandes.

Uamirì. Der mit Urarigift bestrichene Pfeil.

Uapuin assú. Pharmacosycea anthelmintira Miq. Crticaceae. Ph. Rdsch.. 1894, p. 165.

एapuin guassír. Vide Laimì. 
Uaria. Stromanthe lutea Mart. et Eichl. Marantaceae. Ph. Rdsch., New York, 1894, p. 88.

Urinací. Nectandral cinnamoides Nees. Laurareae. Ph. Arch., Milw., 1897.

Uarina. Bauholz in Imazonas.

Uariúva. Vide Tatagiba.

Uariqena. Capsicum Varietiit.

Uarúma. Ischnosiphon Kónma Kcke. Marantaceae. Plı. Rrłsch., New York, 1894, p. 87.

Uarú-rembria, Batuholz in Amazonas.

Ua rìbe. Speise von Mandioca puba, Capsicum, Kuobauch, Salz.

TTassassú. Attilea phalerata Mart. Palmae. Ph. Rdsch., New York, 1889, p. 133.

Uassima. Vide Guaxima.

Uaú-assú. Vide Pallıa branca.

Uaúaxi. Heilpflanze in Amazonas.

Uaússú. Monopterix uaucu Spruo. P'apilionaceae.

Uaússú catinga. Stinkende IT. Monopterix angustifolia Bth. Papilionaceas.

Maxúa. Saccoglottis guianensis Bth. Humiriaceae. Ber. d. Ph. G., Berlin, 1899 , p. 45.

Ua y andy. Vide Jacaré-huiba.

Ubá. Pfeilrohr der Indianer. Gynerium parviflorum Nees. ab E. Gramineae. Ph. Rdsch., New York, 18:)4.

Ubá assí. Grosser U. Vide Paó Perìra.

$\left.\begin{array}{l}\text { Uba-caba } \\ \text { Uba-cahe }\end{array}\right\}$ Vide Uraia do campo.

Ubácaìa, Ubácaya. Costus igneus N. E. Brown. Zingiberaceae. Arch. d. Ph, 1856, p. 229. Ph. Rdsch., New York, 1893, p. 289. Uba-cava. Tupy: uba-Baum, cava-Fett. Vide Bacabal. Ph. Ridsch., New York, 1889, 194.

Ubaia. Saure Beere. Eugenia glomerata Spr. (Tupy: uba-Frucht. aya-sauer) p. 33. Myrtaceae, Ber. d. Ph. G., Berlin, 1903.

Ubaia do campo. Saure Steppenbeere. Eugenia pyriformis Camb., p. 34. Myrtaceae, Ber. d. Ph. G., Berlin, 1903.

Ubaia nitschama, Ubaia muchama. Eugenia obovata, Bg. p. 33. Myrtaceae, Ber. d. Ph. G., Berlin, 1903.

Ubaia-peba. Vide Uvapeba.

Uba-tam, Ubatan. Astronium gracile Engl. Anacardiaceae, Ber. d. Ph. G., Berlin, 1898, p. 164. 
Ubatim. Guaranibenennung für Mais.

Ubatim-caa-eté. Grosser weisser Mais.

Ubatim-eatú. Grosser Maiskolben.

Ubatim-papyra-inhuıai. Maiskolben mit dichtgedrängtstehenden Körnern.

Ubatim-michué. Maiskolben mit den Deckblättern.

Ubatim poróroca. Vide Milho popoca. Ph. Rdsch., New York, 1894 , p. 166.

That inga. Weisser Baum.

Ubatinga piquena. Kleiner weisser Baum.

Bauhölzer in

Chatinga vermelha. Weisser Baum mit rotem Splint.

Uba xì-y. Vide Ubat-y.

Theria. Phyllocalyx strictus Bg. Myrtaceae. Ber. d. Ph. G., Berlin, 1903 , p. 37.

Ubim. Geonoma leptospadi Trl. p. 195.

Tbim-assí. Grosser L. Calyptronomia robusta Trl., p. 265.

[bim-mirim. Kleiner L. Geonoma acaulis Mart., p. 195.

Thim rana. Falscher U. Geonoma maxima, Kth. p. 195.

— Geonoma paniculigera Mart., p. 125.

- Bactris genomoides var. setosa Dr., Rio Grande de Sul. 1888, p. 202.

Ubira-rema / Protium altissimum March. Burseraceae. Ber. U.)ira-siqua $\} \quad$ d. Ph. G., Berlin, 1898, p. 441.

Ubira-tingà. Weisse U. Lucuma fissilis Fr. All. Sapotaceae. Ber. 1. Ph. G., Berlin, 1904, p. 43.

Clú-ai. Vide Lobim mirim.

Ubú-assí

[bussí

Vide Bussì. Ph. Ridsch., New York, 1889, p. 262.

Ubú-caba. Vide Ubaia do campo.

Leabil. Myristica surinamen-is Rol. Myristicaceae.

Tea-pyxingui. Ville Capixingui. Ber. d. Ph. G., Berlin, 1906.

Veara-p ìcì. Fungus.

Iehi. Saccoglottis Uchi J. Hub. Humiriaceae.

Uchi verdadeiro. Echter IT. Licania micrantha Miq. Rosaceae. Ucùcaba, Ueuìba. Das fette Oel der Ueabafriichte.

Ueururé. Fungus. 
Ueu ùba. Myristica sebifera Mart. Myristicaceae.

Ueuúba cheirosa. Wohlriechend IT. Myristica surinamensis Rol. ily risticaceae.

Jcuíba mirim. Kleine U. Myristica paradoxa Schwacke. Myristicaceae.

Uiba. Gynerium saccharoides H. B. Kth. Gramineae.

Uique. Vide Palata und Sapoti-assú.

Uira-repoty. Vide Herva de passarinho.

Uirari. Vide Urari

Uirari-ran a. Falsche U. Strychnos rondeletroides Spr. Loganiaceae.

Iiti. Conepia uiti Bth. Rosaceae.

Uity-kurùb. Vide Oiti-corò.

Uixe. Myristica platysperma Spr. Myristicaceae.

Uixi. Andira amazonum Mart. Papilionareae.

Uliya. Vide Caranai.

Ullucina. Croton thurifer Kth. Euphorbiaceae. Ber. d. Ph. G., Berlin, 1906.

Umari, Umary. Andira inermis H. B. Kth. ?

- Andira spinulosa Mart.

Papilionaceae.

Umari a ma rello. Gelbe U. Poroqueira guianensis Anbl. Rubiacede.

Umari-rana. Falsche I. Conepia subcordata Bth. Rosaceae.

Umari-roya. Violetrote U. Poroqueira sericea Tul. Rubiaceae.

Umbambá, Urùbambá. Desmoncus rudentum Mart. Palmae. Ph. Rdsch., New York, 1888, 1. 265.

U mbarú. Goethia cauliflora Nees et Mart. Malvaceae. Ber. d. Ph. G., Berlin, 1900, p. 271.

Umbarú dos Jardins. Hibiscus cannabinus L. Malvaceae. Eingeführt.

Umbaúba. Cecropia surinamensis Miq., p. 289.

Umbaúba do brejo. Sumpf U. Cecropia lyratiloba Miq.

Umbaúba do mato. Wilde U. C. carbonaria Mart., p. 289.

Umbaúba prateada. Silber U. C. hololeuca Mart., p. 289.

Umbaúba-tinga. Weisse U. C. palmata Willd., p. 289.

Umbaúba vermelha. Rote U. C. adenopus Mart., p. 289.

Urticaceae, Ph. Rdsch., New York, 1891. 
Umbú. Vide Acava. Ber. d. Ph. G., Berlin, 1898, p. 153.

Umbúrana. Vide Imburana macho.

Umburá-púama. Vide Manacan.

Umeri, Lmiri. Humiria Horibunda Mart.. p. 43. $\left.\begin{array}{c}\text { Tmeri balsamo. Balsamischer L. Humi- } \\ \text { rium balsamiferum Anbl., p. } 44 .\end{array}\right\} \begin{gathered}\text { Ber. d. Ph. G.. Berlin, } \\ 1899 .\end{gathered}$

Umeri bastardo. Falscher T. Trattinickia rhoifolia Willd. Burseraceae. Ber. d. Ph. G., Berlin, 1s98, p. 143.

Umki. L-mar. Randia aculeata L. Rosaceae.

Cnamurm. Lauraceae. Baum der Nordstaaten.

Unguraré. Vide Bacalua. Ph. Rdsch., New York, 1s89. p. 193.

Unha de anta. Tapirklaue. Bauhinia fortificata Link. Mimosaceae. Arch. d. Ph., 1860, p. 166.

Unha de boi. Ochsenklaue. B. fortificata Link rar. longiffora Bth. Mimosaceae. In Minas: B. pulchella Bth.

Unha de gambá. Beutelthierkralle. Vide fructa de gamba.

Unha de gato. Katzenkralle. Bauhinia macrophrlla Vog. Mimosaceae.

- Acacia paniculata Willd. Mimosaceae.

- Tanthoxrlum stelligerum Engl. Rutaceae. Ber. d. Ph. G., Berlin, 1899, p. $3 \pm 6$.

Im Sertaó. Mimosa asperata L. Mimosaceae.

Tnha de racca. Kuhklaue. Bauhinia raddiana Bogn. Arch. d. Ph., 185\%, p. 110.

Unha de veado. Rehklaue. Cyphomandra fraxinell: Sendt. Solanaceae.

Lnhúiba. Tide Inga fara-hi.

¿púiba. Bauholz in Amazonas.

Cpéura. Vide Ipé.

Urajúa. Vide Ymira-júa.

Urandi. Vide Anans.

Urapé guassú. Vide Gito em Pernambuco. Ber. d. Ph. G., Berlin. 1901. p. 351 .

Urapénima
Urapora Vide Imira-penima.

Uraúna. Miscolobium riolaceum Vog. Leguminosae.

Urarema. Vide Angelim côco. 
Urari. Pfeilgift der Indianer.

Urari-iba. Giftbaum. Urostigma atrox Miq. Urticaceae. Ph. Rdsch., New York, 1891, p. 164.

Urari-vana. Falscher Giftbaum. Strychnos C'astelnaei Wedd. Loganiaceae.

Urari-üva. Aechter Giftbaum. Strychnos toxifera Schomb. Loganiaceae.

Urari- ïra. Vide Pindaiba urary. Ber. d. Ph. G., Berlin, 1897, p. 464.

Ureba. Calathea tuherosa Vicke. Maranthaceae. Ph. Rdsch., New York, 1894, p. 87.

Uriamem. Sorocea uriamen Mart. Urticaceat. Ph. Rdsch., New York, 1891, p. 165.

Urican a brava. Bactris tomentosa, Palmae. Ph. Rdsch., New Nart.

Uricanga. Geonoma Pohliana Mart. I 1889, p. 195.

Uricaninha. Arundinaria amplissima Nees. Gramineae. Ph. Rdsch., New 'York, 1894, p. 168.

Uricurana. Geonoma fiscellaria Mart. Palmae. Ph. Rdsch., New York, 1889 , p. 195.

Uricurana mirim. Ḱleine U. Croton urucurana Baill. Euphor-

biaceae. Ber. d. Ph. G., Berlin, 1905.

Uricurana piranga Rote U. Vide Uricurana.

Urinaria. Zornia diphyllia Pers. Papilionaceae.

Uritempera. Vide Timboura.

Uriundùra und L rundeùra. Vide Grundeìra. Ber. d. Ph. Ǵ., Berlin, 1898, p. 163.

Urtigas. Nesseln. Vide Ortiga.

Urtiga cipo. Lianennessel. Dillechampia tiliaefolia Lam. Euphor-

biaceate. Ber. d. Ph. G., Berlin, 1905 und 1906.

Urtiga mamai. Melonennessel. Jatropina urens var. brachyloba

Muell. Arg.

Urúbà do caboclo. Marinta furcata Nees. Marantaceae.

Urúba mba. Vide Limbamba.

Urubú - cãa

Urubí - gereo

Ur u bú - goem

Urubú - góene

Aristolochia gigantea Mart. et Zuco. Aristolochiaceae. Ph. Rdsch., New York, 1893, p. 181.

Urubú - caba. Solanum fultum Schrad. Solanaceae. 
$\left.\begin{array}{c}\text { Urucari } \\ \text { Urucari-iba }\end{array}\right\} \begin{gathered}\text { Attalea excelsa Mart. Palmae. Ph. Rdsch., New } \\ \text { York, 1889, p. } 133 .\end{gathered}$ Urúcatú. Amaryllis acuminata Ker. Amaryllidaceae.

Urucú. Orlean. Bixa orellana L., p. 74 Bixaceae, Ber.

d. Ph. G. BerUrucú bravo. \'ilder Orlean. \} Bixa urucurana $\}$ lin, '99, Arch. Irucú-vana. J Falscher Orlean. $\}$ Willd., p. 79. J. Ph., 59, p. Urucú-mirim. Kleines Orlean. Cordia platyphylla Stead. Boraginaceae. Ber. d. Ph. G., Berlin, 1902, p. 132.

Urúcúba. Myristica subsessilis Bth. Myristicaceae.

Urúcúli und Lrúcúy. Vide Alicúli. Ph. Rdsch., New York, 1889, p. 91.

Urucun. Vide Lricurana.

Urúcurana.

Hieronyma alchornoides Fr. All. Euphor-

Urúcurana-piranga. $\} \begin{array}{r}\text { biaceae. Ber. d. Ph. G., Berlin, } \\ \text { Arch. d. Ph., 1859, p. } 176 .\end{array}$

Urúcurana mirim. Kleine L. Croton urucurana Willd. Euphorbiaceae, p. 194.

Urúcuri. T Vide Coqueira cabeçudo. Ph. Rdsch., New York, Urúcuriba. $\} \quad 1889$, p. 90.

Urúcú- rba. Tupybenennung für Mamoeira. Ber. d. Ph. G., Berlin, 1903, p. 163.

Urúçú - hee. Vide Alcassus da terra.

Urukúriaroba. Vide Arikûrioba.

Urumbamba. Vide Úmbambà.

Urumbeba. (Corrumpirtes Tupywort ron Yurúpeba-yu-Dorn, rú - hat, peba - flaches Blatt. - Flaches dorniges Blatt.

Urundeúra.

Trundey-pita. $\}$ Vide Grundeúra.

Urundey-yba.

Urupè. Blutschwamm. Polrporus sanguineus Fr.

Lứû̀ma. Girosses

Lrúpêmba. Kleines $\}$ Sieb von Taquararohr.

Urúpetim. Tupyname für Lophophytum mirabile Schott et Endl.

Balaychoraceae. Ph. Rdsch., New York, 1895, p. 33.

Urúpetim mirim. Kleine U. Lathrophytum Peckolti Eichl. Balaschoraceae. Ph. Rdsch., New York, 1895, p. 33.

Urútim-peba. Pithecolobium Blanchettii Bth. Leguminosae. 
Ittúamba. Guareal tuberculata Vellos. Meliaceate.

Utúapòca. Vide Marinheiro de folha larga. Tupyname.

Utúa úba. \{ Vide Tatúauba.

Ituniea. Bauholz in Bahia.

Ira-assú. Grosse Beere. Vide Iraia do campo.

Uva-aya. Vide Ubaia.

Uva - ípari. Heilmittel in Cearí.

Uva de espinha. Stachelbeere. Berberis lanrina Beth. Berberidareae. Ph. Rer., Milwaukee, 1896, p. 155.

Ivir de gentio. Indianerbeere. Cissinmpelos vitis Vell. Menis permaceae.

Ura do mato. Waldbeere. Cordia scabrilia Mart. Boraginaceate

Ber. d. Ph. (i., Berlin, 1902, p. 132.

Ira da matta. Irwallbeere. C'issampelos rhombifolia Tahl. Menispermaceae.

Ura mirim. Kleine Beere. Vide Ibaia do campo.

Ura pari. P'olygala lanrifolial st. Hil. I'olygalinceae.

Uva-peba. Platte Beere. Hexachlamys humilis Bg. Mrrtaceae.

Ber. d. Ph. G., Berlin, 190:3, pp. 28 \& :34t.

Ura-purana. Śchwarze Beere. Engeniopsis cannatofial Bg. Myrtareale. Ber. 1. Ph. (A.. Bertin, 1903, p. 20. \& 344 .

ITva-pura do campo. Cualea multifloria Mart. Vochysiareane.

Uva-rava. Schlechte Beere. Ternstroemeria-Art.

Ura da serra. Gebirgsheere. C'honlodentron tomentosim R. et

Pers. Menispermalceate.

Ira-tinga. Weisse Bere. Vidle leoito cavallo. Ber. d. Ph. G., Berlin, 1898, 1. 284.

Iraia, Tralha. Samp Bere. ('Tupr. Tba-Frucht, aya-sauer =ubaya). Vide Tbaia.

Ir raia-do campo. Siure Steppenbsere. Psidinm radicans Bg.

Myrtaceae. Ber. d. Ph. G., Berlin. 1903, p. 35:3.

Vide auch Pitanga do campo.

Uraiasinha do campo. Kleine saure Steppenbeere. Vide Itbaiapeba.

Uvalha. Eugenia nvalha Camb. Myrtaceate. Ber. d. Pl. G.. Berlin, 1903, p. 34 .

Uvalha do campo. Vide L'baia do campo.

Uxi. Couepia myrtifolia Bth. Rosaceae.

Vampi. Cookra punctata Retz. Rutaceae. Kultivirt.

Vapores. Vidle Jasmin de S. Jose.

Vaquinha. Terminalial lucidir Hffg. Combretaceae. 
Vara-apia. Vide Graõ de gallo de Parà.

Vareta. Rute. Cypura paludosa Anbl. Iridaceae. Ph. Rdsch., New York, 1892, p. 132.

Vassoura. Besenkraut. Baccharis aphylla D. C. Compositae.

Sida aruta Burm. var. carpinifolia Schum. Malvareae. Ber. d. Pl. G., Berlin, 1900, p. 268.

Sida rhombifolia L.

Corchorus hirtus L. Tiliaceae. Ber. d. Plı. G., Berlin, 1898,

Vide Tupicha und Tupixaba.

Vassoura-assu. Grosses Besenkraut. Alsodeia castaneafolia

Spreng. Violaceae.

Vassoura do campo. Steppenbesenkraut. Dodonaea riscosa

Jacq. Sapindaceae. Ber. d. Ph. G., Berlin, 1902, p. 111.

V assoura vermellaa. Rotes Besenkraut. Dodonaea viscosa

Jacq. Sapindaceae. Ber. d. Ph. G., Berlin, 1902, p. 111.

Vassoureiro. Besenbin?er. I'itherolobium niopoides sipruc. Mimosacene (der Bast).

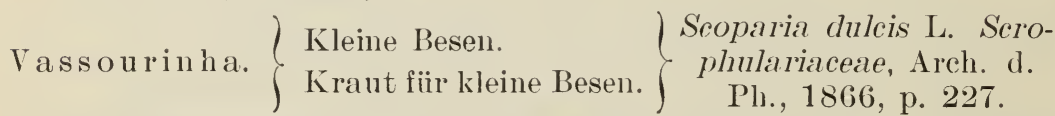

Aulomyrcia ramulosa Bg. Myrtaceae. Ber. d. Ph. G., Berlin, 1903 , p. 24.

Vassourinha de botao. Besenkrautsprossen. Cephalantus scoparius Fr. All. Rubiaceae.

Vassourinha cravina. Nelkenbesenkraut. Oxalis rusciformis

Mik. Oxalidaceae. Ber. 1. Pl.. G., Berlin, 1899, p. 48.

Vassourinha de forno. Backofenbesen. Vide Alfavaca de cobra.

Ber. d. Ph. G., Berlin, 1899, p. 331.

Vassourinha mofina. Heilpflanze in Bahia.

Vatapá. Nationalspeise in Bahia. Masse von Madiocamehl, DendéOel, Capsium, und Fischfleisch.

Velame. Croton campestris Muell. Arg. ) Euphorbiaceae, Croton velame Mart., p. 197.

Velame branco. Weisser V. \}Ber. d. Ph. G., Berlin,

Velame branco in Rio Grande do Sul. Macrosiphonia longiflora Muell. Arg. Apocynaceae.

Velame branco do mato. Weisser wilder $\mathrm{V}$, Julocroton fuscescens Baill.

Velame cabelluda. Haariger V, p. 199. Julocroton fuscescens Baill.

Velame bravo. Wilder. V. Julocroton triqueter

Euphorbiaceae, Ber. d. Ph. G., Berlin, 1905. var. atrorufus Muell. Arg. 
Velame branco do campo. Weisser Steppen $\mathrm{V}$. Croton campestris var. genuini Muell. Arg., p. 195.

Velame do campo. Stepen V. Croton fulvum Mart.

Velame do cheiro. Wohlriechender T. Vide.

Emphorbiaceae, Ber. d. Ph. G., Berlin, 1905.

\section{Alamplioreira, p. 19).}

Velame da matta. Irwald V. Vide Velame da purga.

Velame miudo. Kleiner $\mathrm{V}$. Oxalis divaricata Mart. et /uce. Oxalidaceae. Ber. d. Ph. G., Berlin, 1899, p. 46.

Velame preto do campo. Sichwaryer Steppen V. C'roton campestris var. nigricans Baill. Enplorlliaceale, Ber. d. Ph. G., Berlin, 1905, p. 197.

Velame da purga. Purgir $V$. Julorroton triqueter var. genuinus Muell. Arg. Ber. d. Ph. ('x., Berlin, 1!)05, p. 199.

Velame rana. Falscher V. Iponnoea tomentosa Pohl. F'onvolvulaceare.

Velludinho. Kleines Samtkraut. Guettardia nrngnensis Cham. et Schl. Rubiaceale.

Velludo. Simtkraut. Guettardal uruguensis Cham. et Sichl. Rü biaceae.

Velludo de espinho. Dorniger Samthanm. Chomelia liirsuta Gard. Rubiaceae.

Velludo macho und Marmelada macho. Alibertia elliptica Schum. Rubiaceae.

Tentona, auch Schibatan, Schubatan. Tantana contracta var. grandiftora Urb. Humiriaceae. Ber. d. Ph. G., Berlin, $1899, \mathrm{p} 45$.

Verbasco. Wollkraut. Verbascnm blattaroides Lam. Scrophnlariaceae. Vile auch Barbasco. Arch. d. Ph., 1857, p. 238.

Verga de Jaboti. Jabotirute. Erisma calcaratum Warm. Vochysiaceae.

Verga do mato. Heilpflanze in Bahia.

Veronica. Dalbergia monitirial L. Fil. Palpilonaceale.

Veronica da terra. Calpraria biffora L. Seroplntariaceae.

Veroróla. Vicle Ucuúba.

Vetiver. Andropogon squarrosus L. Gramineae. Ph. Rdsch., New York, 1894, p. 110.

Viba. Dickes Rohr. Indianische Benennung für Zuckerrohr.

Videira. Weinstock. Vitis vinifera L. Ampelidaceae.

Vina. Iriartea phaeocarpa Mart. Palmae. Ph. Rdsch., New York, 1889. 
Vinagre ira. Essigpflanze. Vide Carurù azedo. Ber. d. Ph. G., Berlin, 1900.

Tinda-caa. Alpinia nutans Rosc. Zingiberaceae. Ph. Rdsch.. New York. 1893, p. 288.

Vinhatico. Vochysi, rufia Mart. Tochysiaceate.

Acacia maleolens Fr. All. )

Plathymenia foliosa Kth. $\}$ Mimosacea.

Vinhatico amarello. Gelber V. Echirosperum Balthasarii Fr. All. Caesalpineaceae.

Vinhatico do campo. Steppen V. Enterolobium ellipticum Bth. Plathymenia retirulata Bth. Caesalpineaceae.

Vinhatico para esteios. Piosten V. Piptarlenia paniculata Btl. Mimosaceae.

Vinhatico fllor de alğodaõ T. mit Baumwollbliite. Enterolobinm Schomburgkii rar. Glasionvii Bth. C'aesalpiniaceae.

Vinhatico testa de boi. Ochsenschällel V. Tupsname ist: Sitbingengura. Vide Vinluatico amarella. Pond. sp. $=0.657$.

Tinheiro. Vide Paó de tucano.

Tinheiro do campo. Tochysia thrrsoidea Pohl. Tochrsiaceae.

Violeta. Teilchen. zufolge des violetten Splints. Bauholz in l'eara, p. sp. $=1,120$.

Violetar. Tiola odorata L. Fiolaceae.

Violeta in Parì. Silla alpestris sit. Hil. Malvaceale. Ber. d. Ph. G., Berlin, 1900, p. 26(;.

Violeta do mato. Wildes V. Sirla panirulata L. Malraceate. Ber. d. Ph. G., Berlin, 1900, p. 266.

V isgueira. Togelleimbaum. Parkia platycephala Bth. Mimosaceate. Viura. Wittwenblume. Petraea subserrata Cham. Verbenaceae. Arch. d. Ph.. 1s(;2, p. 139; Ber. d. Ph. G., Berlin, 190t, p. 478.

Viurinha. Kleine Wittwenblume. Petrapa denticulata Schrad.

Arch. d. Ph., 1862, p. 139); Ber. (l. Ph. G., Berlin. 1904, p. 478.

Voadeira. Vide ('arurì amargeso.

Vuarame. Bohrr. Helicteres vuarame Mart. Sterculiaceae. Ber. d. Ph. G., Berlin. 1900, p. 59.

Yúba. Gynerinm saccharoides H. B. Kth. Cramineae.

Vúrapia. Flechtenbaum. Celtis aculeatis'w. ( Loticaceae. Ph. Rdsch., C'eltis acuminat: S.

\} New York, 1892, p. 34 .

Wacapì. Fonacalpona anrelicana Anbl. Papilionactae.

Wapá. Eperua leucantha Bth. Caesalpiniaceae.

Xanxim. Crathea Sanchin Mart. Cyatheaceae.

Xeri-caa. Ottonia proquinqua Kth. Piperaceae. Ph. Rdsch., New York, 1894,1$) .28 \%$.

Ieque-xeque. Cardiospernum corindum L. Sapindaceae. Ber. d. Ph. G., Berlin, 1901, p. 451. 
Xequiri. Vide Inquiri.

Seringueira. Vide Seringueira.

Xiló. Vide Giló.

Ximbár a, Ximbó, Ximbúra. Vide Timbouva.

Ximbaúva parda. Brame X. I'ithecolobinm Langsdorfii Bth.

Mimosaceae.

Xiquechique in Pernambuco. Vide 'Tange-tange.

Xiquechique do sertao. Vide C'hique-xique.

Xiri-caa. Ville Seringueirat branca.

Xiriúba. Vide Mangue vermelho.

Xiriúbeira. Ville Herva de chumbor.

Xiry-caa. Vide Xumby-catena.

Xixi. Vide Teipóca.

X ìcìba. Vicle sicuúbar.

Xumby-caena. Piperomia hederaceal Miq. I'iperarceate.

X и́ и́. Bauholz in Amazonas.

X ихu. Vide Chńr hín. Ber. d. P’h. G., Berlin, 1904. P. 169 .

Yaborandi, Yamborandi, Yanguallandi. Ottonia anisum Spreng. I'iperalcear. P'h. Ralsch., New York, 1894, p. 286.

Yandiroba. 'Tuprname fiir Carapa. Ber. d. P’h. G., Berlin, 1901, 1. $35 \% 3$.

Ya raratia. Gual'aniname fïr Jalacatia.

Yata-i. Cocos Yatr Malt. Palmate. Ph. Rulsch., New York, 18৪8, p. 96,1889, p. 91 .

Yatay. Cocos Palraglarirensis Barb. Rodr. Palmar.

latay-guassú, Grosie I. Scheelia quadrispermal Barb. Rodr. Pallmate.

Ya tay-pendì. Angel Y, Cocos sapidal Barb. Rodr.

Yatay-pong. Liegende Y. ， Diplotheminm Anizetzii Barb.

Yatay rasteira. Kriechende Y. $\}$ Rodr. Palmae.

Yaupé-apóna. Vogelpfanne. Vide Forno.

Íb-metarí. Vide Cajazeiro. Ber. d. P. G., Berlin, 1898, p. 168.

Y ba-raynha. C'onnaris P'atrisii Planch. Connaraceate.

Ýbá-tatá. Tupybenennung für Batatá.

Ybira-paye. Tupyname für Aguay und Balsamo.

Ybira-rema. Vide Ibarema.

Yciy und Ycica. Vide Jcicariba.

Yeica-antan. 'T'upyname für Paì de breo.

Y-cipó. Vide Cipo de carijó.

Ycó, Yikó. Capparis Yco Mart. et Eichl. Cayparidaceae. Ber. d. Ph. G., Berlin, 1898, p. 45.

Yeary-cumajé. Tupyname für Anthurium oxycarpum Poepp. Araceae. P̉. Rdsch., New York, 1896, p. 279. 
I'gar's ì. Feuillea trilobata L. Cucurbitaceae. Ber. d. Ph. G., Berlin, 1904, p. 330.

Ygary. Tupyname für Mongubeira.

Ymbiri. Vide Imbiri.

Ymira, Ymyra, Y bira. Tupybenennungen für Baum, Strauch.

Ymira-ataia. Vide Craveiro de Maranhaó.

Ýmira-êtm. Süsses Holz. Tupyname für Buranhem. Ber. d. Plh.

G., Berlin, 1904, p. 43, Ph. Rdsch., New York, 1888, p. 30.

Y mira-iriba. Tupyname für farbiges Holz. Vide Araribá.

Ymira-itá. Tupy fïr Steinholz. Vide Paó ferro.

Ymira-Kyinha. Tupy für Pieffer. Vide Paó eravo.

Y mira-jùa. Tupy für Beerenbaum. Vide Joazeiro.

Y mira-pagé. Tupy für Heilkïnstler-Baum. Vide Balsamo (Myrospermam).

Ýmira-pariba. Bogenliane. Vide Cipó de caboxolo.

$\left.\begin{array}{l}\text { Ymira-pima } \\ \text { Ymira-pinima }\end{array}\right\}$ Schwarzes Holz. Vide Paó de rainha.

Ymira-pòca. Brechbares Holz. Myrsine umbellata Mart. var. acutifolia Miq. Myrsinaceae.

Y'mira-piranga. 'Tupiname: Rotes Holz. Vide Paó Brasil.

Ymira-puturana. Guaraniname für Páo ferro.

Ymira-reme. Vide Gorarema.

Ymira-siqua. Vide Ubira-siqua.

Ymira-taya. Piefierholz. Bauholz in den Nordstaaten, nach Dr.

Nicolaú Moreira. Lauraceae.

Ymira-tinga. Weisses Holz. Vide Guaracica.

Ymira-una. Tief schwarzes Holz. Miscolobium violaceum Vog.

Dalbergia Spruciana Bth. Papilonaceae.

Yourou-pari. Oureupana guianensis Anbl. Rubiaceae.

Yoyoca. C'acoucia coccinea Anbl. Combretaceae.

Ypadí. Vide Ipadí.

Ypé

Iperóba $\}$ Vide Ipé.

Ypeúba

Y pú. Tupyname für Batata da purga.

Yquetaia. Herpestes monniera H. B. Kth. Scrophulariaceae.

Yquetaia de brejo. Sumpf Y. $H$. strictil Schrad. Scrophulariaceae.

Yquetaia. Wohlriechende Y. H. depressa Bth. Scrophulariaceae. Yri. Vide Brejaúba. Ph. Rdsch., New York, 1888, p. 262.

Yrino. Vide Cuticaem.

Yrupé. Tupyname für Milho d'agua. 
Yrumbéba, Yurumbeba. Opuntia brasiliensis Ham. ('actaceae. Y tó. Vide Gitó in Pernambuco. Ber. d. Ph. (x., Berlin, 1901, p. 350. Yuca. Yucal gloriosal L. Lilialceale. Kultivirt.

Zabueaja. Vicle Sapucair.

Zaburro. Entkïrnter Maiskolben.

Zambôa. Vide Limâo. Varietät.

Zambueiro. Vide Fava do mato.

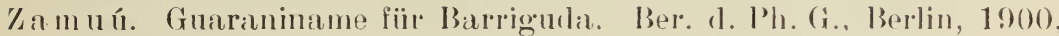
p. 159 .

Zanzì. Tupyname für ('há Inglez. Ber. d. Plı. (i., Berlin, 1900. p. 268.

Zapotá-assí. Vide Silpota gramde.

"zapota-mirim. Vide Sapoti.

Zarza. Tupyname für Salsaparilha.

Zarzanora. Vide Juqueri.

Zimbro. Wachholder. Juniperus communis L. I'onifmate. In den

Siidstaaten kultivirt.

Zopyró. Clinopodium vulgare L. Labbiatae.

Zoró. Speise von Guiabòtrïchten und Ḱrabben.

Zóró. Ternonial obovata Less. I'ompositale.

Zubragi. Vide Subragi.

Zuynandy. Clusia Burchelli Engl. Guttiferate.

\section{CORRECTIONS.}

1) $12,1.31$. pauciflora

p. 20 , 1. 5 . foliosa

p. 27 , 1. 15. Diplusodon

p. $31,1.23$. camarao

p. $37,1.28$. roxa

1. $42,1.26$. Cahabú

p. 4:3, 1. 21 . subinermis

1. 22. Elaes

1) $45,1.21$. Calabúra

1 46, 1. 13. Couepia

i. $1 \%$ capoeira

p. $51,1.24$. nirim

p. 52, 1. 30. Caonin

v. $54,1.3$. camellao

1. 29. megistory

p. $55,11.19 \& 26$. pavão

p. $60,1.24$. boceta

1. 27 Nanthium

p. $61,1.17$. Cybistax

p. 6r, 1. 3. Trixis

p. $74,1.34$. Cipo

p. $80,1.13 .1$ 1 $\overparen{\operatorname{aoz} u}$

p. $83,1.27$. Torresia

1. 84, 11. 20 \& 21. Cupiùba 1. 33. Curaùa p. $ง 6,1 . \tau$. Feĩo

1. 1s. Ebano

1). 8?, 11. 5 \& 6. corno 1. 6 . adena

p. $93,1.21$. aponouita

1. 37. Folha

p. 95, 1. 7. Formigueira

p. $97,1.34$. atrophischer

1. 3.. oauassì

1. $98,1.17$. Garfìana

1. 19. Garùiba

p. $100,1.5$. Gingìba

1. 31. utaiba

1) 102, 11. 18 \& 19. Grâs

1. 1ร. Baùna

1. 3(). Brõmelia

1) 107, l. 1:3. Guarubù lamarell,

l. $17.10 x 0$

1. 22. Guarùvêma

1. 24. camio

p. $108,1.11$. Vide Babo

p. $1(0), 1.20$. roya [de porco

p. $110,1.25$. botao
1. :32. Fortuna 1). $111,1.21$. sìn veolt $\mathrm{ns}$ 1). 112, 1. (i. dutrò

1. 15. Jоаิ Paes

p. 117, 1. ๙. Ahonay

1. 11. Guacao

1. 37. tructo

1. $120,1.34$ xix

1). 121, l. 5. fogo

l. ?. Calliandra

p. 123, l. 13 . amarillo

1). 126,1 25. Jacaré

1. 35. mehl u. Rohrzucker und ir asser

p. $127,1.2$. Jalion and

1. 128, 1. 4. Agriao

p. 129, l. 14 , Jarbao

p. $133,1.23$. Jo

p. 135, 1. 25 . Clusoia

p. $137,1.5$. dasyantha

1. 30. Hoock 
1). 1:38, 1. 21. Larang $\widetilde{a 0}$ 1. 24. Bacupari

p. 139 , 1. 8. Tellerwascher 1. 27. Maranhao

p. $1+0$, il. $3,5,7 \& 10$. Liman

1. 5. I. galega L. f.

1. 16. Mutisia

p. 141 , 1. 20. hypolenca 1. 195, 1. 5. Pirâ

p. 143, 11. 2 and 4 . Maçan 1). $200,1.6$. Nessel

1. 2. brejo

1. (i. sabaก

1. 13. ipou

I. 16. Macaùbà

1. 20. Bacoparido

p. $144,1.7$ taboleira

p. $145,1.20$. Tincenco

1. 34. Nalmęnew$\sinh :$

1. :36. Botica

p. $146,1.18$. macao

1. 20 óurado

ii. 31 to $: 34$. Mamâ

1. $14 \pi, 1.5$. Minas

11.35 \& 37 ) Manacao

p. 148, 11. 1 \& 2 f Manac

p. 149. 1. 20. Minas

l. 33. Tinborao

p. 1.50, 11. 7,9$), 10,11$ and 13. Mangericao 1. 3.5. jandan

p. $151,1.24$ grande

p. $1.53,1.5$. macaัก

1. (j. maman

1. 4. melão

1. $156,1.20$. Malmajura 1. 37 . sertao

p. 1ร8, 1. 13. L,ombùgueira 1. 30 Sichneli tïrltend.

p. 159, 1. 16. Sambacuim p. $160,1.24$. agrarium ii. 26 , et ser]. Me1ลก

1. 3:3. moca

1). 161, 1. 6. Cuiarana

p. 163, 1. 13 . Muira

p. 1633,1 , 1 , sertao....Моric

p. $16 ; 6,1.8$. Murriao

p. $17: 3,1.22$. Ortigao

p. $174,1.35$ anaio

p. $178,1.3$. Paineira

1. 8. camarao

p. 17 !) 1. 35. Јоаัо

p. $181,11.2$ \& 5. pareira 1. 35. Ceutrolobium

p. $182,1.9$. C'raveira

1. 13 roxo

1. 29. Mureci

p. $183,11.1$ \& 23 . Tabebuia.

1. 18. tueanorum

1. 22. Cassia aponconiea.

1. 33. Tinhorao

р. 20×, 1. 32. praia
1) $184,1.15$. parao

p. $188,1.10$. Machixa

p. $190,1.13$. Bicho

p. 191 , il. 1 \& 5.$\}$ Pichão

p. $192,1.30$. Solanum

1. 193, 1. 34 . chapadensis

. $194,11.5$ et seq. l'in-

[hã)

il. $)$ \& 11. Guill-

1. 30. Kirschen-

[purganz

p. 201, 1. 1. Excaecaria

1, 11. seatìa

1. 2:3. Guabiroba

1, 27. Quarenta

p. 202, l. 4 . caryophyllus

p. 203, 1. 14 . branca

1). $205,1.7 .1901$

1. (). $\mathrm{Dc}$

1. 10. Bumelia

1. 12. Capituliflora

l. :32. Blugió

p. $206,1.2 .2 \mathrm{i} q$

l. 7 . caperonoides

1. 14. Warm

l. 19. Rachador...

1. 20 . Siuaximi

1. 29. babeira

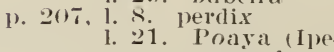
(a) viluha)

1. 32. Japecallgil

1. 33. Piretro

p. 209, 1. 7. Mrrtaceate

1. !). brunioides

1. 25 . I). ('.

p. 210, 1. 1:3. Sohlecht

1. 14. ('áa-op)ia

i. 16. $110 x \mathrm{imm}$

1. 18 (

p. 211. 1. 5. ('amb.

p. $212,1.13$. Sch $n \mathrm{~m}$

l. 16. seyodaplirlla

1. 24. Scamann numla

$$
\text { ....Herva }
$$

1) 213.1 .8 . peptocalyx

1. 14. rasteiro

l. :33. camblatal

1). $214,1,7$. Seringueiros

1. 9. $K^{\text {th }}$

ii. 17 \& 1 s. Sitrike out all between parentheses.

1. $: 32$. Lecytlopsis glabra ('amb

1. 3.). Poepp

1) 215, 1. :3. Saputa

1. 14. Acrodicli-

11. 21 \& 22 . guya[nense

1. 23. Physocaly-

1. 25. Pennatu

1). $216,1.5$. amatrella

1. 7 . cumeat:

1. 13 . brancia

1. 21. rallal
1. 2.5. vermalha

1. 29. Quentlel

1. 31. Strike out tromiticum

1. 217,1 . 1. Didspanax

Clongepetiolatum

1. 11. Strike ont Kautschuk liefernol.

1. 29). „1marella

1. 34 . ferrea....leios

1) $218,1.20$. sucuuba

1. 2.). colhurnum

i. 31. rana

1. 3:3. Fumo

1. 36. Tabebuia

1. 219 , 1. 11 . estreita

1. 2.2. tridy

1). 220, 1, 16. fasciculata

1. 20 . lacerolaei

p. 2.21, 1. 8. Timbouva

l 2:3. cipo

1. 29. Tanchagem

1. 30. Desf

1. 32 . cala

1. $222,1$. 5. Lilnceta

1. 2:3. branca

1. 28. guîanense

1. 30. Tapirila

1. 36. Tapiranga

1). $223,1.2$. fiir

l. 6. fellea

1. 18. Tapiximgui

1. 31. Riedleelànil

1). $224,1.1$. mirin

1. 4. Taquara

1. 10. Burchlielii

1. 11. longeglume

1. 16. bezerro

1. 2ง. rana

1. 330. Tarampaba

1. 36;. pasto

i. 38 . ('itharexylon

1) $225,1.15$. Zoplioriceate

1. 21. suplatavilaris

1). 226, 1. !) licsuta

1. 3.2. gracilis

p. $227,1.17$. pato

1. 2ล. Balthazarii

1) 22я. 1. 1؟. Timbó

1. 31. bratro

1. 34. Lianel

p. 229, 1. 1. guassú

1. 10. minda

1. 15. peixa

p. $231,1.8$. Anil

1. $233,1.19$. ussu

11. '2:3 and 33. ralla

1. 35. Tremoços

p. 234, 1 15. Ville Chu-

1). $2: 37,1.6$. Sanand $\hat{\imath}$

1. $\%$ Solm

i. 10. Cajnkernen

1. 21. Seoparia

1. 34. Turama...

p. $239,1.3$. ITarimacú

[Hot $\angle$

1. 7. Lariquena

l. S. A rouma

i. 17 Sprue

1. 2(). Uaxúa

p. 240, 1. 21 . rana

p. 241 , ii. 11 \& 21 . Conepia

p. 242, 1. 36. Crapoca

p. 243, l. 4 \}rana

ii. 33 \& 35. Bala-

[nophoraceat 


\section{MONOGRAPHS.}

1. Popular German Names. This popular pamphlet has been revised twice by its anthor, Dr. Fr. Hoffmann.

0.50

2. Reagents and Reactions known by the names of their authors. Based on the original collection of $\mathrm{A}$. Schneider; revised and enlarged by Dr. Julius Alts chul; translated from the German by Dr. Richard Fischer, Asst. P'rofessor of Practical Pharmacy at the Iniversity of Wisconsin. Although imperfect in many respects, this compilation has proven a convenient aid in the laboratory and on the desk. A revision is now in progress. Out of print.

3. Popular Scandinavian Names. A compilation of popular Swedish names of drugs and medicines by Harold Bruun, with formulas for the preparation of a number of preparations not generally found in American reference works. This list is also being revised. Copies still on band can be had for

$\$ 0.15$

4. Early Phages in the Development of Pharmaceutical Legislation in Wisconsin. An account by Edward Kremers of the evolution of the first local pharmacy law in Wisconsin with the documents on which the account is based. Pamphlet, pp. 43 . $\$ 0.50$

5. Some Cubau Medical Plants. While collecting plunts in Cubu during the year 1895 and 1896, Prof. R. Combs had Ifis attention directed to numerous plants of the island used as domestic remedies. Painphlet, pp. 20.

$\$ 0.15$

6. History of the Art of Distillation and of Distilling Apparatus. By Oswald Sclıreiner. Pamphlet, pp. 59, with 65 illustrations.

$\$ 0.35$

7. The Crude Drugs and Chemicals of the United States Pharmacopoeia (1890) and the Preparations Into Which They Enter. By W. O. Richtmann. Pamphlet, pp. 55. Now being revised.

$\$ 0.25$

8. Progress in Alkaloidal Chemistry, 1903. A collection of abstracts by Dr. H. M. Gordin. Pamphlet, pp. 40.

$\$ 0.30$

9. The Sesquiterpenes. A monograph by Oswald Schreiner. Brochure, pp. 130.

$\$ 1.00$

10. Progress in Alkaloidal Chemistry for 1904. By H. M. Gordin. Brochure, pp. 94.

$\$ 0.70$

11. The Volatile Oils: 1904. By I. W. Brandel. Brochure, pp. 51. Intended primarily as a text for students.

12. The Balance. By I. W. Brandel and Edward Kremers. Brochure, pl. 49 , with 48 illustrations.

0.35

13. A Review of the Literature on the Estimation of Alkaloids for the Year 1905. Brochure, pp. 17. By W. A. Puckner. 0.20

14. The Naming of Carbon Compounds. By W. A. Puckner. Brochure, pp. 17.

0.20

15. Volksbenennungen der brasilianischen Pflanzen und Produkte derselben in brasilianischer (portugiesischer) und der von der Tupisprache adoptirten Namen. Von Theodor Peckolt. 


\section{MONOGRAPHS. - Continued.}

(In courec of preparation.)

-Progress in alkaloidal chemistry for 1905. By H. M. Gordin.

-The Volatile Oils: 1905. By I. W. Brandel.

-Percolation. A brief historical account, followed by a statement of general principles, a complete bibliography and laboratory exercises. Inteuded primarily for students of pharmacy. By I. W. Brandel and Edw. Kremers.

-The Volatile Oils: 1901-1903. By 1. W. Brandel. The manuscript is practically completed. When published, these annual reviews will constitute a complete supplement to G.-H.-K. "The volatile oils." See the second cover page.

\section{BIBLIOGRAPHIES.}

1. Chemical Biography of Morphine. From 1875 to 1897 , with an index of authors and subject index. By H. E. Brown. Pamphlet, pp. 60.

$\$ 0.40$

2. Santonin. Bibliogruphy, with abstracts of methods of production etc. From 1830 to 1897 . By $\mathrm{A}$. Van $\mathrm{Z}$ waluwenburg. Pamphlet. pp. 11.

$\$ 0.10$

3. Bibliography of Apiol. From 1855 to 1896 . By A. Van Zw aIuwenburg. Pamphlet, pp. 4 . $\$ 0.05$

4. Bibliography of Spirit of nitrous ether, and ethyl nitrite. Up to 1899. BF W. O. Richtmann and J. A. Anderson. Brochure, pp. 180.

$\$ 1.00$

5. Bibliography of aromatic waters. From 1809 to $1900 \mathrm{incl}$. By W. O. Richtmann. Brochure, pp. 219. $\$ 1.00$

In addition to the pamphlet form, these bibliographies will be found very convenient for card catalogues which can be kept up to date as indicated by the following fascimile reproduction of such a card.

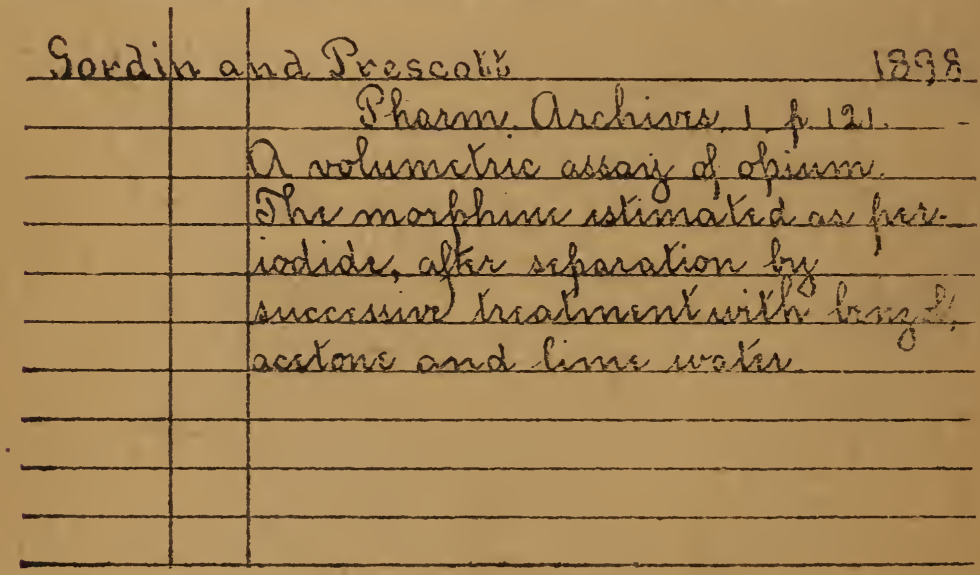







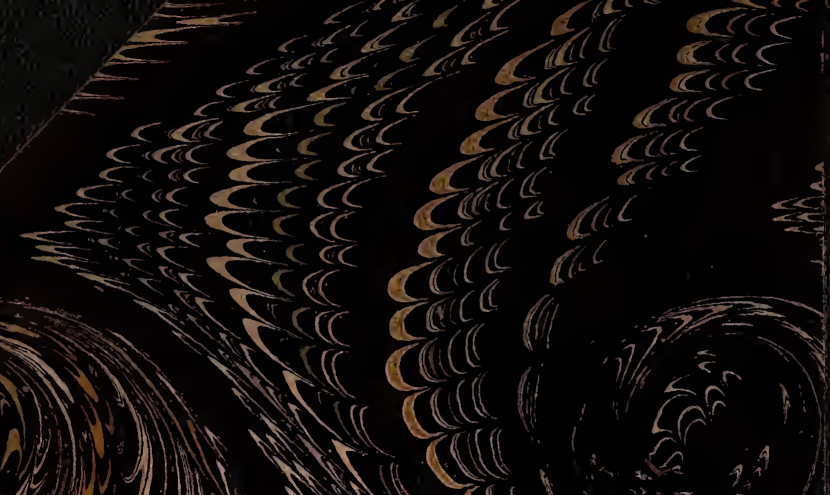

$(6,3))$ )

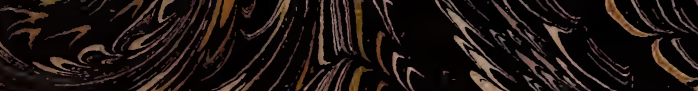
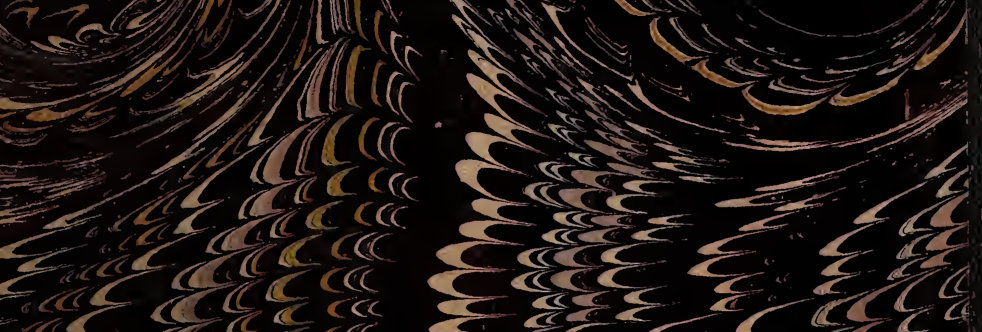

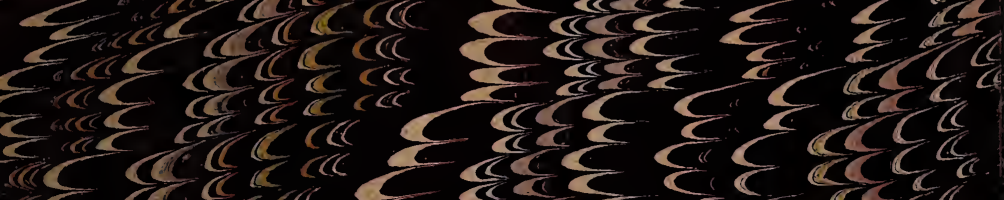
ह हता

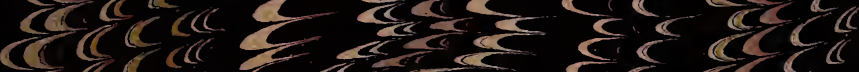
(c) हैyc(I) = रीखा c(di) $\rightarrow$ से है। foce - 1 (1)

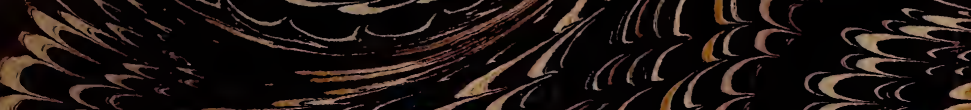

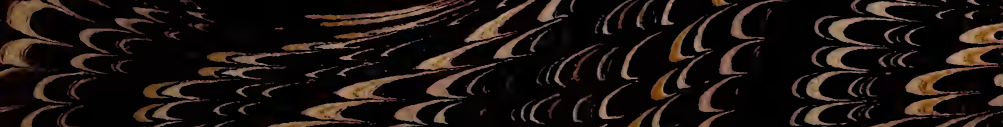

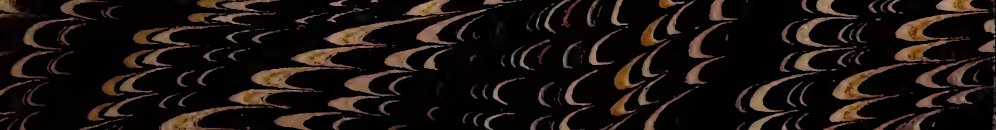

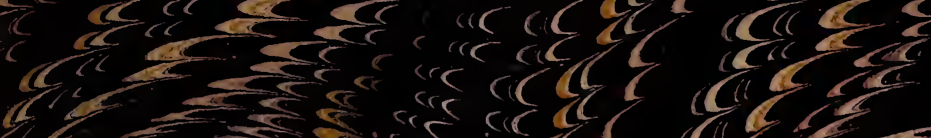
T. -5 -

$$
\begin{aligned}
& \angle-\infty \\
& \text { if re } \\
& \text { - inivers } \\
& \text { \&s } \\
& \text { Q }=
\end{aligned}
$$

